Markus Osterhoff

\title{
Wave optical simulations for $x$-ray nano-focusing optics
}
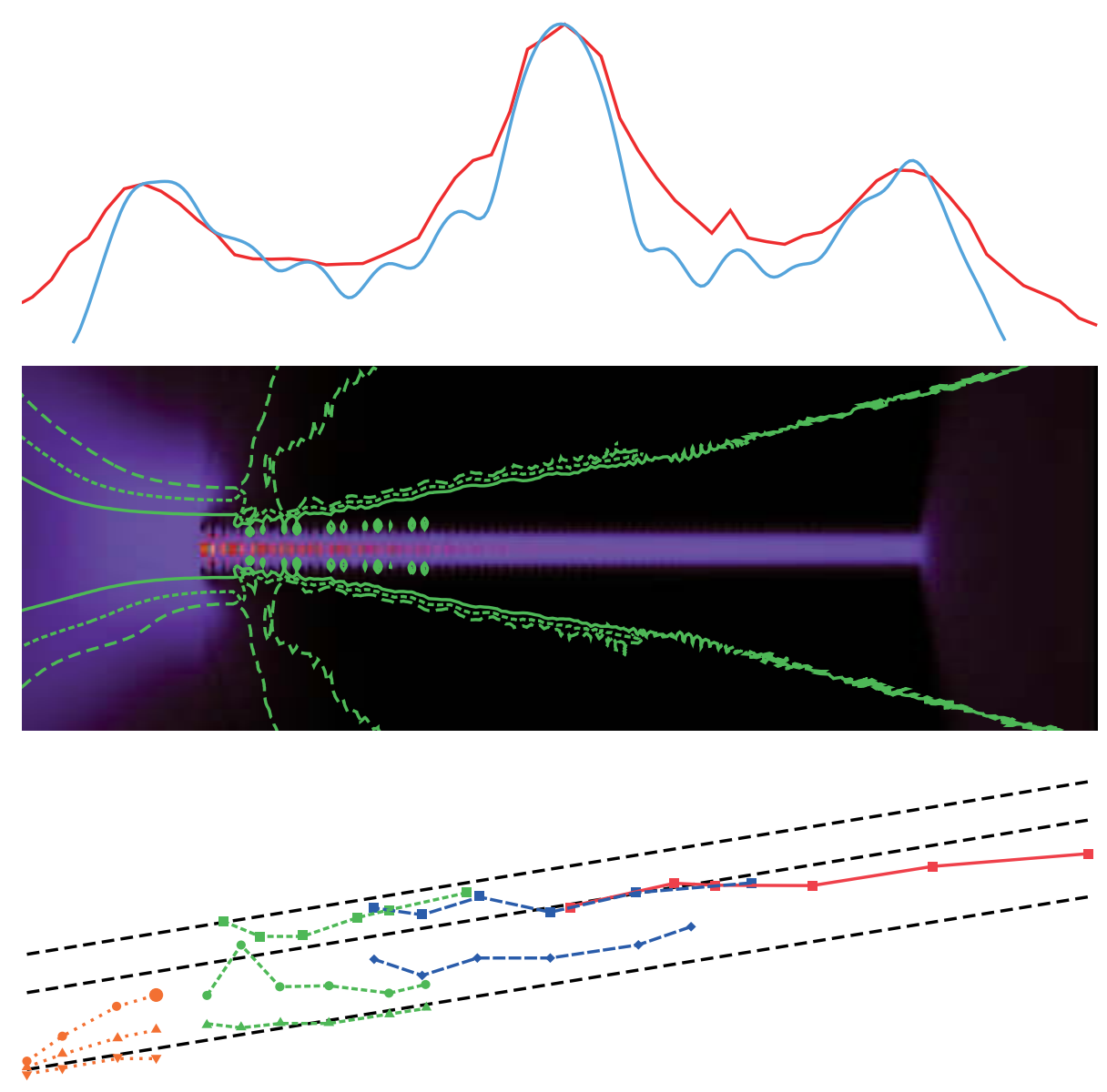

Markus Osterhoff

Wave optical simulations

for x-ray nano-focusing optics

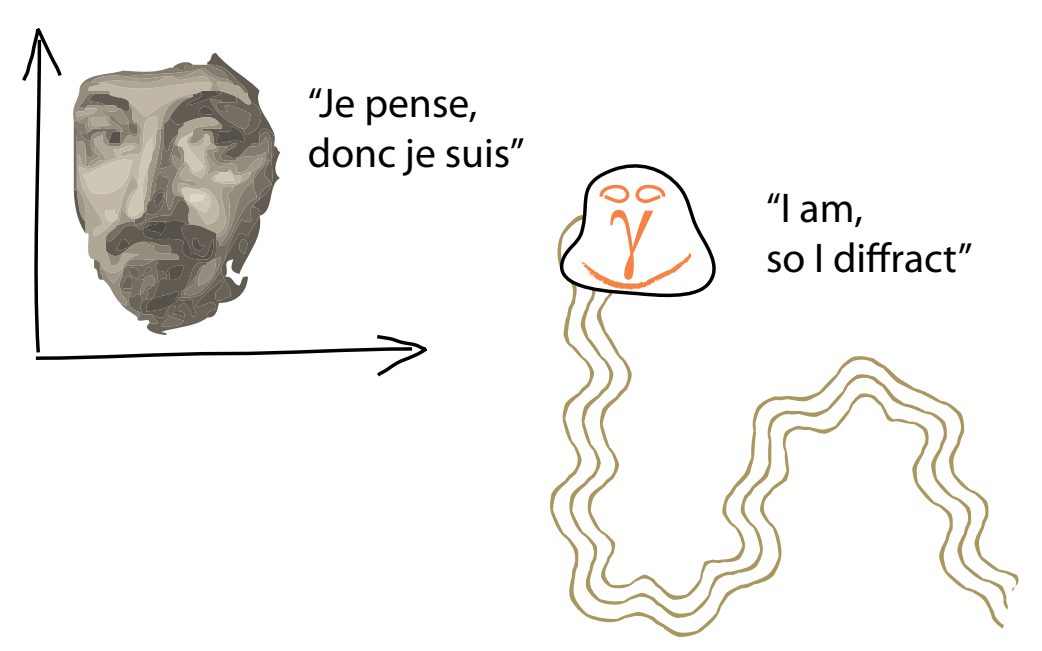





\title{
Wave optical simulations for $\mathrm{x}$-ray nano-focusing optics
}

\author{
Dissertation \\ zur Erlangung des mathematisch-naturwissenschaftlichen Doktorgrades \\ „Doctor rerum naturalium“ \\ der Georg-August-Universität Göttingen
}

\author{
vorgelegt von \\ Markus Osterhoff \\ aus Warstein
}

Göttingen, 2011 
Mitglieder des Betreuungsausschusses:

Prof. Dr. Tim Salditt, Prof. Dr. Arnulf Quadt, Prof. Dr. Sarah Köster Referent: Prof. Dr. Tim Salditt

Koreferent: Prof. Dr. Rainer G. Ulbrich

Tag der mündlichen Prüfung: 


\section{Contents}

1 Introduction 1

2 Partial Coherence 3

2.1 Introduction . . . . . . . . . . . . . . . . . . . . 3

2.2 Degree of coherence . . . . . . . . . . . . . . . . . . . . . . . . . . 5

2.3 Stochastic phase . . . . . . . . . . . . . . . . . . . . . . . . . . . 16

3 Simple Mirrors $\quad 19$

3.1 Introduction . . . . . . . . . . . . . . . . . . . . . . . . . . . . . 19

3.2 Theory. . . . . . . . . . . . . . . . . . . . . . . 22

3.3 Partially coherent focus simulations. . . . . . . . . . . . . . . . . . . . . 28

3.4 Defocus properties. . . . . . . . . . . . . . . . . . . . . . . . . . . 42

$4 \quad$ Multilayer Mirrors $\quad 47$

4.1 Introduction . . . . . . . . . . . . . . . . . . . . . . . . . . . . . 47

4.2 Takagi-Taupin equations in curved ML geometry . . . . . . . . . . . . . . . . . 50

4.3 Generalised Bragg conditions . . . . . . . . . . . . . . . . . . . . . . 60

4.4 Solving the Takagi-Taupin equations . . . . . . . . . . . . . . . . . . . 66

4.5 Integrated reflectivity. . . . . . . . . . . . . . . . . . . . . . . . . . 73

4.6 Results. . . . . . . . . . . . . . . . . . . . . . . . . . . 80

5 Waveguides $\quad 93$

5.1 Introduction . . . . . . . . . . . . . . . . . . . . . . . . . . . . . 93

5.2 Analytical degree of coherence. . . . . . . . . . . . . . . . . . . . . . 94

5.3 Numerically propagated degree of coherence . . . . . . . . . . . . . . . . . 102

5.4 Combined optics. . . . . . . . . . . . . . . . . . . . . . . . . . . . 104

6 Conclusion $\quad 107$

6.1 Summary. . . . . . . . . . . . . . . . . . . . . . . . . . 107

6.2 Outlook......................... 108 
A Tabular derivation . . . . . . . . . . . . . . . . . . . . . . . . . . . 111

B Families of rays . . . . . . . . . . . . . . . . . . . . . . . . . . . . 113

C Implementation details of XMLS . . . . . . . . . . . . . . . . . . . . . . . . . . 115

D Implementation details of TT . . . . . . . . . . . . . . . . . . . . . . . . . . . 129

E Command-line interface. . . . . . . . . . . . . . . . . . . . . . . . . . . . . . . 139

F Web-based interface . . . . . . . . . . . . . . . . . . . . . . . . . . 141

G Remote control via HTTP . . . . . . . . . . . . . . . . . . . . . . . 145

H Modified Bragg condition . . . . . . . . . . . . . . . . . . . . . . . . 149

I X-ray optical constants daemon . . . . . . . . . . . . . . . . . . . . . 151

J Comparison: GCC 4.3 vs. 4.1 . . . . . . . . . . . . . . . . . . . . . . . . . 153

Bibliography . . . . . . . . . . . . . . . . . . . . . . . . . . . . 155

Index . . . . . . . . . . . . . . . . . . . . . 163 


\section{Chapter 1}

\section{Introduction}

The primordinal object of this thesis was to obtain an analytical and numerical description of focusing multilayer (ML) mirrors, that are mainly used at the European Synchrotron Radiation Facility (ESRF) in Grenoble, France. ML mirrors provide high quality $x$-ray beams for investigation on a large variety of specimens. A new analytical treatment for curved $\mathrm{ML}$ mirrors will be given in chapter 4 , together with numerical results. As a natural and important prerequisite, I have started with simulations of total reflection mirrors, illuminated by pointsources (chapter 3 ). Seeking a comprehensive model to account for partial coherence and finite source-sizes, I came up with a stochastic model of ensemble averages, cf. chapter 2. Using this model, coherence filtering by $\mathrm{x}$-ray waveguides is shown in chapter 5 .

Physics is the science that tries to understand how nature behaves. Optics is an important topic of fundamental research, but also provides us with tools to study other systems. After Wilhelm Conrad Röntgen had discovered "eine neue Art von Strahlen" [1], these x-rays soon were used as probes in medicine and science, long before their true nature as light waves was unravelled [2]. Over the last few decades, large-scale facilities providing scientists from many different disciplines with synchrotron radiation have emerged. Compared to laboratory sized laser sources, these beams are faint and weak. Good optics are crucial, but hard to manufacture.

With this thesis, new theoretical insights and numerical tools are given to the scientific community to further improve focusing optics based on curved focusing ML mirrors. A comprehensive model for propagation of partial coherence is put forward that shall help in development and testing of new algorithms for a variety of imaging techniques using coherent $\mathrm{x}$-ray beams.

This thesis presents results of a collaboration between the Georg-August-Universität Göttingen and the ESRF Grenoble. 
CHAPTER 1. INTRODUCTION 


\section{Chapter 2}

\section{Partial Coherence}

\subsection{Introduction}

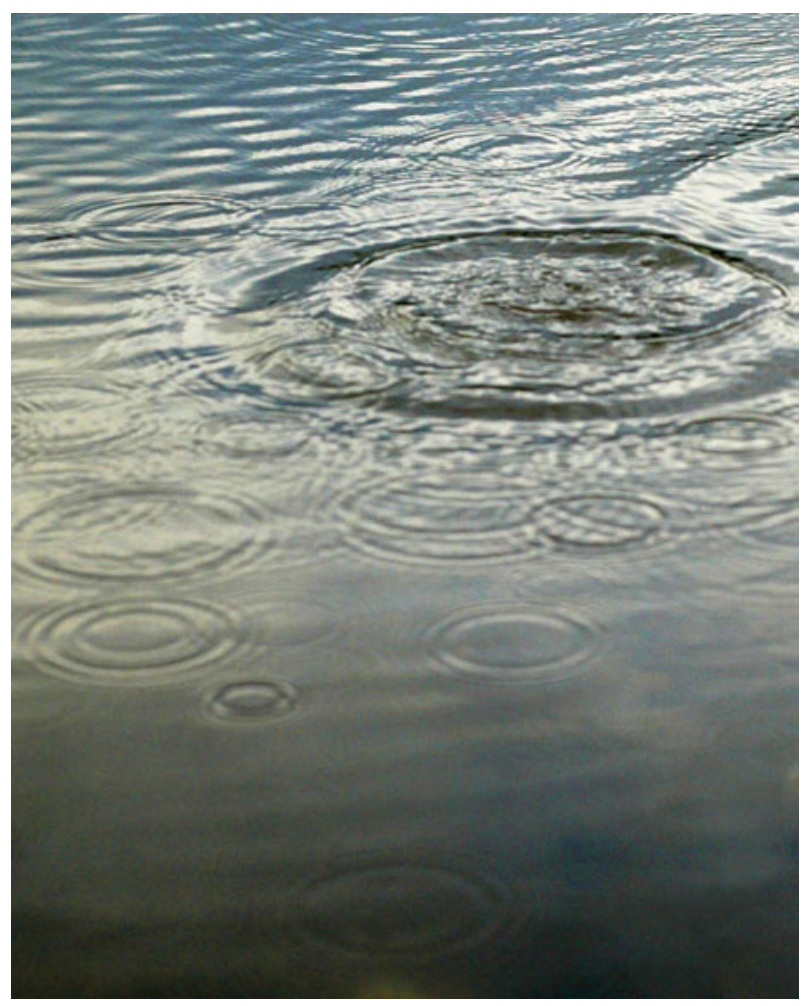

In geometrical optics, intersecting light rays simply experience linearly enhanced intensity. Wave optics, on the other hand, introduces the concepts of phase relations and interference: Not intensities, but complex amplitudes are the protagonists for linear additions*. Depending on the phase difference, light plus light can produce shadow in some places and more light in other places. Of course the total energy of the fields does not change [3].

In elementary courses, optical fields often are simplified to monochromatic plane waves $[4,5]$. In that case the addition of amplitudes is always correct. In less idealised models the fields are made up of polychromatic wave-packets with short lifetimes, finite both in the temporal and the spatial domain. This results in stochastic fields where the interference effects fluctuate in time $[6,7]$ and often are not detectable at all ${ }^{\dagger}$.

Imagine the following gedankenexperiment: a "random plane wave" with a stochastic direction illuminates a double-slit. Stochastic direction means that the wavevector $\vec{k}(t)=$ $|\vec{k}| \hat{k}(t)$ has a constant length $|\vec{k}|$, but changes randomly direction $\hat{k}(t)$ on some time-scale. These random directions imply varying phase relationships. The interference fringes start to wiggle according to the random process. If the phase-relations change faster than a typical detection time scale, the interference fringes start to wash out. The "visibility" of the fringes, as defined later, will at some point tend to zero, so a roughly constant intensity will be measured along the detection plane, if the phase-relation at the two slits fluctuates

\footnotetext{
*We do not consider any non-linear phenomena here.

$\dagger$ †X-ray waves oscillate with $\gtrsim 10^{18} \mathrm{~Hz}$; even relatively "slow" fluctuations of $\sim 10^{12} \mathrm{~Hz}$ are hard to detect electronically.
} 
within $\pi$ or more. A more sophisticated analysis would result in the functional description of the visibility for certain models, viz. for a stochastic description of the phase-randomness.

These phase fluctuations usually are explained by two causes: temporal coherence deals with not-monochromatic waves, spatial coherence deals with not-point-like sources which show randomly fluctuating phase-relations of waves emerging from different points. Monochromatic filtering enhances temporal coherence, while spatial filtering (i.e. with pinholes) enhances spatial coherence. Well stabilised laser sources exhibit full coherence over the whole beam size and time-scales of seconds. At present, comparable sources do not exist in the x-ray regime, but envisioned at free electron laser sources (FELs). "Monochromatic" filtering for $\mathrm{x}$-rays is typically based on Bragg reflections and achieves relative bandwidths of $\Delta \lambda / \lambda \sim 10^{-4} \ldots 10^{-8}[8]$. In the spatial domain, modern synchrotron sources are different in horizontal and vertical direction. This often is simplified by an elliptical distribution of electrons around their orbit $[9,10]$. At least in vertical direction "rather coherent" beams can be expected, while in the horizontal direction the degree of coherence is well below one [11].

In the present treatment we will focus on spatial coherence and assume that the light-field is quasi-monochromatic but fluctuating $[6,7]$.

Let us have a closer look at the typical coherence experiment which we will consider later.

The basic set-up we will analyse is depicted in figure 2.1: an x-ray beam emitted from a synchrotron source (bending magnet, undulator, etc.) impinges on a focusing mirror and converges to the focus. In the focus or defocus, a double-slit is positioned, and the diffraction pattern in the far-field zone is recorded. This will show either strong or weak oscillations; the visibility ${ }^{\ddagger}$ can then be measured for different slit separation distances and as a function of defocus. This simple picture stands as an example of a great variety of more sophisticated and complex set-ups, including Talbot interferometry [12-16]. Also, these measurements are not limited to focusing mirrors, but can be applied to multilayer mirrors, beams filtered by $\mathrm{x}$-ray waveguides, refractive lenses, multilayer Laue lenses, and to unfocused beams, as well.

Based on the double-slit experiment, an easy simulation scheme using the method of stochastic fields is described in section 2.2. We will show how to model partial coherent intensity distributions in the near-field and far-field of an x-ray focusing mirror, based on a very general approach which can be used for a large class of sources. Not only the partially

\footnotetext{
$\ddagger$ The visibility $v$ only equals the modulus of the degree of coherence $|j|$, if the two pinholes are illuminated with equal average intensities.
} 
(a)

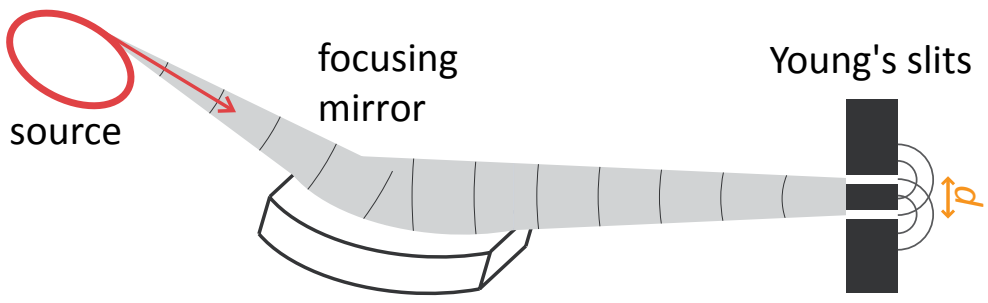

(b) Diffraction pattern

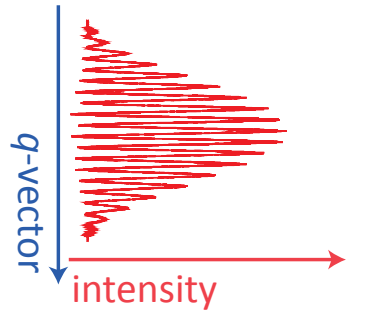

(c) small $d$-coherent

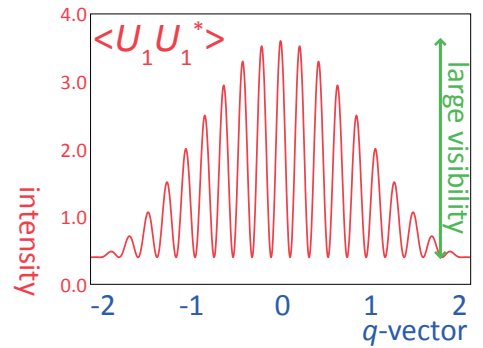

(d) large $d$-incoherent

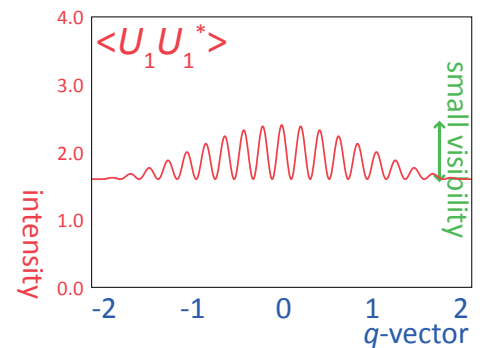

(e) Degree of coherence $|j(d)|$

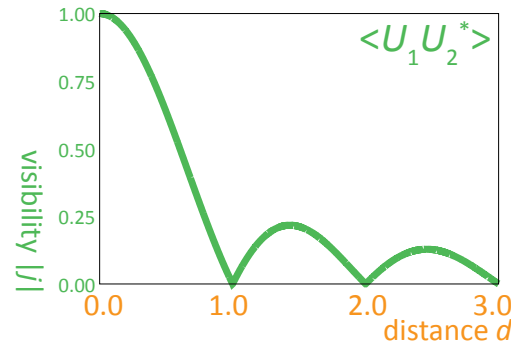

Figure 2.1: Schematic illustration of the degree of coherence, $|j|$, defined as the visibility $v$ of fringes. In (a), a basic diffraction experiment of a double-slit, illuminated with a pre-focused synchrotron radiation $x$-ray beam, is shown. The typical diffraction pattern I $(q)$ of the double-slit in the far-field as a function of reciprocal vector $q$ is shown in (b); depending on the slit distance $d$, the diffraction pattern is rather pronounced (c) or averaged out (d); from the "visibility of fringes", the modulus of the complex degree of coherence, $|j(d)|$, can be obtained.

coherent intensity, but also the complex degree of coherence - and hence the usually most interesting quantity, the visibility - can be calculated. Finally, we give a generalisation of the Gouy phase anomaly [17] in focused fields for partially spatial coherent illuminations.

The technique developed here will then be used in chapter 3 to simulate not only focusing, but also coherence properties of such optics. Later on we will use both this numerical and an analytical approach to model coherence filtering effects of x-ray waveguides (chapter 5).

\subsection{Degree of coherence}

In this section we put forward a numerical model to simulate the classical double-slit experiment depicted in figure 2.1. This model is based on stationary stochastic optical fields [6] and we assume a fluctuating monochromatic - and spatially incoherent $-x$-ray source. For our model we have a typical undulator source of $3^{\text {rd }}$ generation synchrotron facilities in mind; but the idea of the following considerations is common for classical light sources and can in principle be applied to bending magnets, $x$-ray tubes, and modern micro-focus lab sources as well. 


\subsubsection{Chaotic sources}

Such chaotic light sources in general show intensity fluctuations on different time scales:

- The event that produces light is of short duration. An undulator only emits when an electron beam passes through; $x$-ray tubes emit characteristic lines when a vacancy of an electron shell is re-filled; thermal atoms in light bulbs or in a discharge lamp only emit for a short time interval. In between, light might be emitted from other points in the source; after some delay, the considered point may emit again, but with a random phase relation.

- The light producing event is of quantum mechanical nature, hence intrinsically random. Photons are distributed according to some probability density, depending on their state of quantum optical coherence (most commonly, Poissonian statistics, but also subor super-Poissonian are possible [18]).

- The "driving force" of the emission, i.e. an electric current, changes on different time scales due to thermal noise (Johnson noise) [18], electron orbit variations in synchrotrons, or large-scale envelopes (like decreasing ring currents or - most dramatically - beam dumps).

- Focusing, beam path guiding, filtering, and detection are quantum mechanical processes and hence introduce additional randomness.

There exist sources that diminish some of these variations; conventional lasers can produce stable beams on the seconds-scale, seeded free electron lasers are proposed as sources with comparable properties. In this work we will not discuss such "perfect" sources, but confine to "classical", noisy sources. It can be expected that correlations between these emitters can be incorporated using correlated random coefficients.

Since the phase-relations are subject to rapid and random changes on a time-scale of the coherence time $\tau_{c}$, interference effects might not be accessible if the detection process integrates (in other words: averages) over larger time scales $\tau_{\text {meas }} \gg \tau_{c}$. The "strength" of the interference is then determined by the partial coherence.

Consider a synchrotron source with a manifold of electron bunches, even coming up with independent sub-structures: These will emit billions of uncorrelated wave-packets. Each one possesses a new random phase-relation with respect to the previous one, introducing fluctuations on many time scales that are not accessible to detection. More strictly, this idealisation of vanishing correlation only holds for so-called Lambertian sources that emit uniformly in all directions. Since the electrons (or positrons) circulate at ultra-relativistic 


\section{$\begin{array}{lll}\text { (a) electron orbit } & \text { (b) directed synchrotron radiation } & \text { (c) far-field }\end{array}$}

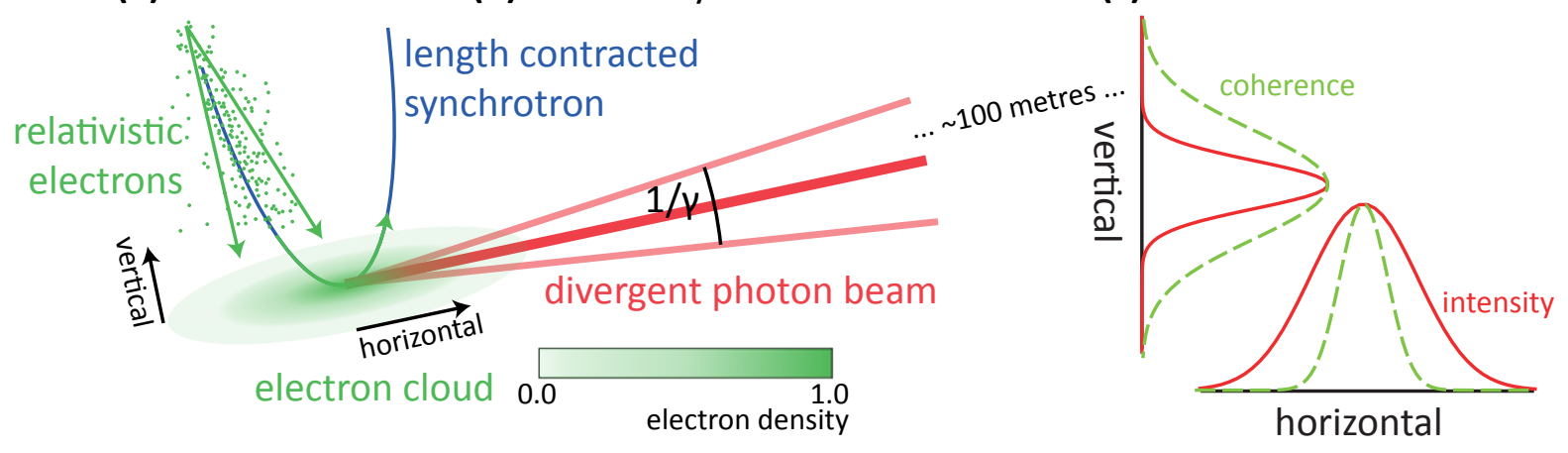

Figure 2.2: Coherence properties of modern synchrotron radiation sources, following the Gaussian-Schell model [11,22]. The circulating electrons often are approximated with an elliptic distribution around their orbit; their relativistic velocity yields a directed radiation cone. On the right, the farfield intensity (red lines) and degree of coherence (green dashed lines) is shown for the horizontal and vertical direction.

speed (common relativistic energies are on the order of $6 \mathrm{GeV} \sim 10^{4} m_{e}$ ) [10], synchrotron sources mainly emit into a cone of small angular spread, $\theta \sim \gamma^{-1} \sim 10^{-4}$ [8]. Here, $\gamma$ is the relativistic Lorentz factor. Then a considerable correlation is present already in the source plane.

Analytical calculations show that third generation synchrotron sources can be regarded as nearly coherent in the vertical direction, while only partially coherent in the horizontal direction [11]. Beamlines with lengths of hundred to even one thousand metres have been built (not only) to increase coherence properties by propagation $[8,19-21]$. See figure 2.2 for an illustration.

\subsubsection{Simple definitions}

After these introductory remarks let us now describe light fields in terms of their coherence properties. Consider a monochromatic light wave (plane or spherical wave, focused beam etc.) of frequency $\omega$, described by its complex amplitude ${ }^{\S}$

$$
U(\vec{r}, t)=U(\vec{r}) e^{-i \omega t}
$$

Dealing with spatial coherence properties of quasi-monochromatic sources, we will only use the spatially-dependent part $U(\vec{r})$ now. For brevity let us simply write $U_{1}:=U\left(\vec{r}_{1}\right)$ and $U_{2}:=U\left(\vec{r}_{2}\right)$. Then the "usual intensity" as a measure of energy flux density may be defined

\footnotetext{
$\S$ The complex amplitude is defined as the analytical signal in [6].
} 
by

$$
I_{1}:=U_{1}^{*} U_{1}, \quad I_{2}:=U_{2}^{*} U_{2},
$$

where $U^{*}$ denotes the complex conjugate of $U$. Different definitions involve a constant factor depending on the units of measurements. Since such a factor would not lead to different results in our treatment, we set it to unity. The intensity is the energy flux density and related to the Poynting vector $[3,23]$.

This ordinary intensity is a function of one space-point. More generally, the equal time mutual intensity is usually defined as

$$
J_{1,2}:=U_{1}^{*} U_{2}
$$

Introducing this time-independent quantity we restrict the discussion to stationary fields in the wide sense, where the mutual intensity only depends on $t_{2}-t_{1}$; working with the equal time mutual intensity, $t_{2}-t_{1} \equiv 0$.

Obviously, $I(\vec{r})=J(\vec{r}, \vec{r})$.

Now the complex degree of coherence $j_{1,2}$ between the space-points $\vec{r}_{1}$ and $\vec{r}_{2}$ is defined as the normalised mutual intensity $J$,

$$
j_{1,2}:=J_{1,2} \times\left(J_{1,1} J_{2,2}\right)^{-1 / 2} .
$$

It may be shown that for quasi-monochromatic fields (i.e. small-bandwidth waves with a small spread $\Delta \omega$ around a mean frequency $\bar{\omega}, \Delta \omega \ll \bar{\omega})$ the following relation holds:

$$
j_{1,2}\left(t_{2}-t_{1}\right)=j_{1,2} e^{-i \bar{\omega}\left(t_{2}-t_{1}\right)} .
$$

Hence the visibility $v=|j|$ is not affected by a small bandwidth [7]. This encourages us to use this model for not-monochromatic beams as present in experiments.

\subsubsection{Modelling and simulation}

Spatial incoherence is the result of a finite source size, or of a source emitting different modes subject to fluctuations. We will mainly focus on the first aspect. Let us discretise the finite source by a number of independent point-sources $s_{n}$. These sources generate optical fields $u_{n}$ in a region of interest, perhaps behind focusing or filtering devices. If all point sources would emit the light waves in perfect correlation, we could simply add all fields $u_{n}$ to get the resulting amplitude,

$$
U_{\text {coherent }}:=\sum_{n} w_{n} u_{n}
$$


where the real coefficients $w_{n}$ represent some envelope function for the point sources.

But in the case of a chaotic source, each virtual point-source independently emits wavetrains of finite duration with arbitrary phase-relations to its own history and its neighbours. If we could measure for a sufficiently short time interval, we could resolve a rapidly oscillating speckle pattern.

In the cases we want to discuss in this thesis, the detector integrates over a rather long time period (on the order of milli- to kilo-seconds). Then an incoherent sum of many realisations of a coherent, but stochastic summation of the basic fields $u_{n}$ is measured. The coherent stochastic summation gives realisations $U$ of the stochastic optical field:

$$
\begin{aligned}
U_{\text {speckle }} & =\sum w_{n} c_{n}^{\text {rand }} u_{n}, \quad c_{n}=a_{n} e^{i \varphi_{n}}, \\
a_{n} & =\operatorname{rand}(0,1), \quad \varphi_{n}=\operatorname{rand}(0,2 \pi) .
\end{aligned}
$$

Again, the real coefficients $w_{n}$ are an envelope function for the point-sources, while the complex random coefficients $c_{n}^{\text {rand }}$ have a different amplitude and phase for each realisation of the stochastic process. The amplitude are given by a flat distribution in the interval $[0,1)$, the phase by a flat distribution in $[0,2 \pi)^{\top}$.

A source with intrinsic coherence properties could be modelled by random coefficients $c_{n}$ with a "coupling" to near-by point-sources, e.g. using a Gaussian distribution centred around $c_{n-1}$, similar to Markov chains. X-ray undulator sources show a finite, but still very small coherence length already in the source plane [11] that is even longer for FEL-sources (free electron lasers). But such correlations have not been used in the following analyses.

The detector integrating over a long time interval is now modelled by an averaging of many stochastic realisations:

$$
U_{\text {stoch }}=\left\langle U_{\text {speckle }}\right\rangle=\left\langle\sum w_{n} c_{n}^{\text {rand }} u_{n}\right\rangle
$$

So we assume an ergodic system where the time-average (detector) is the same as the ensemble average (stochastic realisations). For convenience we restrict the discussion to the complex degree of coherence between two points in a lateral plane; this plane is assumed to be perpendicular to the optical axis, at a distance $x$ from the focal plane. One reference point shall stay fixed on the optical axis at $y=0$, whilst the "probe" point is placed in a distance $d$. The complex degree of coherence, $j_{x}(d)$, as defined in equation (2.1), is then

\footnotetext{
IIn computer codes, the GNU library function double drand48 (void) (POSIX.1-2001) has been used to obtain pseudo-random numbers based on linear congruentials.
} 

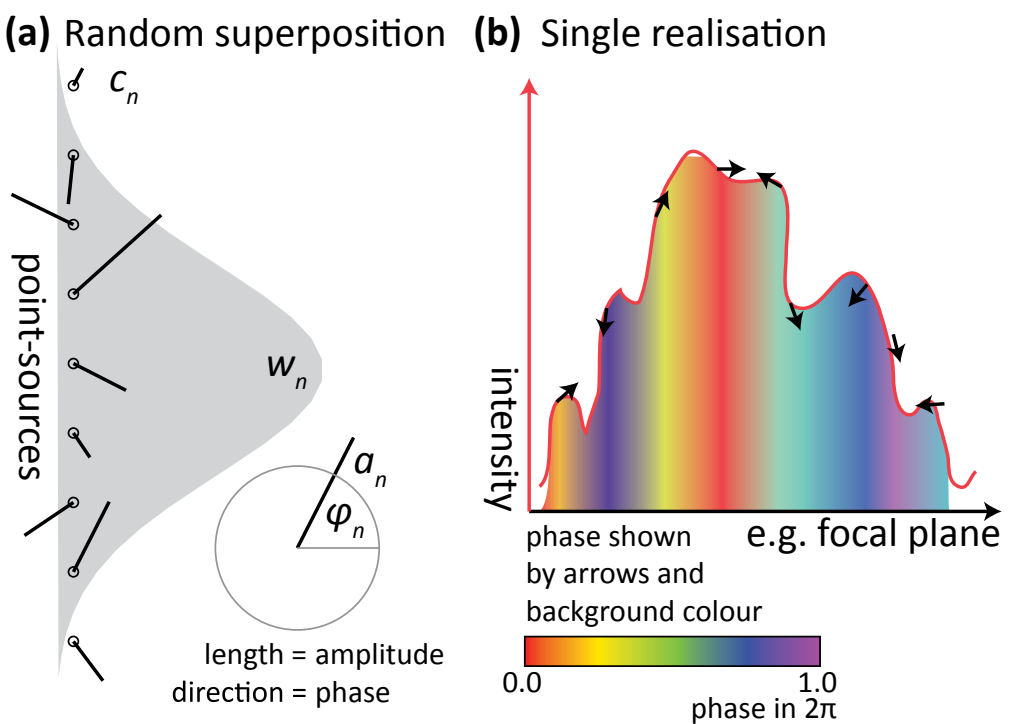

(c) Stochastic ensemble

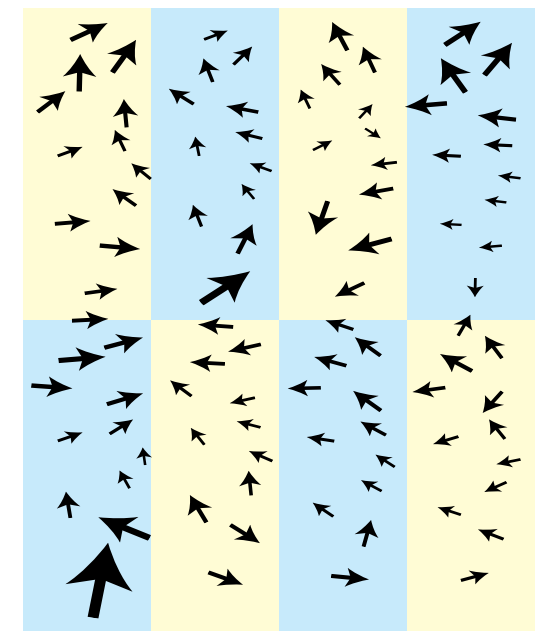

Figure 2.3: Illustration of the simulation model. In (a), the amplitude and phase relations of a single stochastic realisation is shown, with a hypothetical interference pattern in (b). Part (c) shows a stochastic ensemble of eight source realisations.

evaluated with $J_{1,2}=\left\langle U_{1}^{*} U_{2}\right\rangle$ :

$$
j_{x}(d)=\frac{\left\langle\left(\sum w_{n} c_{n}^{*} u_{n}^{*}(x, y=0)\right) \cdot\left(\sum w_{n} c_{n} u_{n}(x, y=d)\right)\right\rangle}{\sqrt{\left\langle\left|\sum w_{n} c_{n} u_{n}(x, y=0)\right|^{2} \cdot\left|\sum w_{n} c_{n} u_{n}(x, y=d)\right|^{2}\right\rangle}} .
$$

This stochastic method to model the coherence properties of optical fields is illustrated in figure 2.3. In part (a), individual point-sources, indexed by $n$, are shown; they are weighted by an envelope $w_{n}$. Each realisation of the random process is characterised by a tuple of complex random amplitudes and phases $c_{n}=a_{n} \exp \left(i \varphi_{n}\right)$, illustrated the arrows. The lengths correspond to the instantaneous intensity, the direction to the phase. The optical field originating from the point-sources is pre-calculated and thence superposed using the tuple $\left(c_{n}\right)$, resulting in one "shot" of the fluctuating field, see part (b). In (c), an ensemble of tuples $\left(c_{n}\right)_{e}$ is shown.

Now we will look at the two limiting cases, $j=0$ and $j=1$.

Fully coherent field If $j=1$, the stochastic realisations always show the same phase relations, it is sufficient to just add the amplitudes:

$$
j=1 \quad \Rightarrow \quad U=\sum w_{n} c_{n} u_{n}, \quad I=|U|^{2} .
$$


This corresponds to a single speckle pattern emerging from a stochastic ensemble, that consists only of one realisation.

Fully incoherent field There is no correlation of the fields; so it is appropriate to just add the intensities:

$$
j=0 \quad \Rightarrow \quad I=\sum w_{n}^{2}\left|u_{n}\right|^{2} .
$$

Without any correlation no experiment can be thought of that shows interference; hence, the complex amplitude $U$ is not defined.

Van Cittert-Zernike theorem Fully coherent sources are approximated by mono-modal stabilised lasers; perfect incoherence is the limiting case of an infinitely large and uncorrelated source. Due to propagation, even two perfectly uncorrelated point-sources show partial coherence if observed under a small angle (i.e., from a distance much larger than their separation). This is a result of the van Cittert-Zernike theorem $[6,24,25]$; the complex (equal-time) degree of coherence $j_{x}\left(y_{1}, y_{2}\right)$ for a perfectly uncorrelated source in a distance $x$ is given as the normalised Fourier transform of the source's intensity distribution $I$ :

$$
j_{x}\left(y_{1}, y_{2}\right)=\int I_{x=0}\left(y^{\prime}\right) e^{-i k\left(\hat{y}_{2}-\hat{y}_{1}\right) y^{\prime}} \mathrm{d} y^{\prime} / \int I_{x=0}\left(y^{\prime}\right) \mathrm{d} y^{\prime} .
$$

\subsubsection{Examples}

Mono-modal laser A perfect, ideal laser produces a fully coherent wave field. This is possible despite its finite source size, because "all atoms emit in phase" - or in other words: they emit in the same mode - and thus are fully coherent:

$$
j(d) \equiv 1
$$

Multi-modal laser In a laser with several modes excited, one subset of the atoms emit in phase with respect to each other, with a random phase relative to another subset of atoms. These different modes then add incoherently, the degree of coherence is not constant, but smaller than unity for large slit separations. The dependency has been demonstrated experimentally for a multi-modal laser in $[26,27]$. We come back to a description of coherence in terms of modes when discussing $x$-ray waveguides in subsection 2.2.6. 
Chaotic Light (light bulbs, x-ray tubes, stars, etc.) In the limiting case where every atom emits in its own mode they are statistically independent and perfectly incoherent. This is the regime of the van Cittert-Zernike theorem; partial coherence arises due to propagation. In the special case of a one-dimensionally slit, the degree of coherence is given by a sincfunction" [28].

\subsubsection{Computational Methods}

In this subsection we discuss useful techniques to numerically evaluate (2.2). The complex degree of coherence $j$ is given as the normalised ensemble average of the mutual intensity $J$. Now $J$ is the coherent sum of pre-calculated fields $u_{n}$, multiplied with (pseudo) random numbers $c_{n}^{\text {rand }}$ and envelope coefficients $w_{n}$. Many summations have to be carried out, if $j_{x}(d)$ is to be evaluated in a large number of defocus planes $x$ and for many pixels in distances $d$ to the optical axis.

The computational time scales linearly in each of the following parameters. We list useful ranges of important parameters, that can be well matched to analytical results as discussed later.

1. Number of point-sources: Typically, we will use about 100 to too point-sources. Note that as many basic fields $u_{n}$ have to be pre-calculated by propagating $x$-ray fields. This might take several seconds (for focusing mirrors) or minutes (for multilayer mirrors or waveguides).

2. Size of ensemble: ensemble averages of less than 1000 evaluations have proven to result in strong numerical noise; tests show that about 10000 stochastic realisations should be used. Together with (1), this results in about three million complex additions per pixel.

3. Resolution, $N_{x} \times N_{y}$ : the problem grows linearly with the number of pixels. The full information, $j\left(x_{1}, y_{1}, x_{2}, y_{2}\right)$, would even need $\left(N_{x} \times N_{y}\right)^{2}$ steps. Since we have decided not to correlate different defocus planes with each other, and we assume that the correlation does only depend on distance $d=y_{2}-y_{1}$, not individual points $\left(y_{1}, y_{2}\right)$, complexity both in calculation and visualisation is reduced. Depending on resolution, $3 \cdot 10^{6}\left(N_{x} \times N_{y}\right)$ stochastic realisations are needed to give useful estimations for the mutual intensity $J$.

\footnotetext{
$\|_{\operatorname{sinc}}(x):=\sin (x) / x$
} 
4. Average of $|j|$ : Numerical experiments have shown that a direct evaluation of (2.2) still suffers from noise for distances $d$ with small coherence, $|j(d)| \lesssim 0.5$. If correlations are small, the ensemble average sums up complex numbers of similar modulus but arbitrary phase. This proves to be just as difficult as adding up real numbers of similar magnitude, but opposite signs; relative errors add up vastly. To overcome this problem, an "outer average" of the modulus of $j$, each calculated by its own stochastic ensemble, is taken:

$$
\tilde{j}_{x}(d)=\left\langle j_{x}(d)\right\rangle
$$

About ten to twenty evaluations give considerable results, increasing the overall computational costs by one order of magnitude.

In conclusion: with the proposed numbers, the evaluation of $j_{x}(d)$ of $10^{5}$ pixels needs about $10^{2} \ldots 10^{3}$ teraflops, plus the propagation of 100 to 300 point-sources to a ROI of $10^{5}$ pixels.

A direct propagation of the mutual intensity with generalised Fresnel-Kirchhoff integrals is often even more expensive. Consider a source plane of $10^{2}$ points which is to be propagated to 100 defocus planes consisting of $10^{3}$ points each. Hence the mutual intensity needs the evaluation of $10^{13}$ complex exponentials for the phase terms, which each typically need $10 \ldots 100$ flops, hence $10^{3} \ldots 10^{4}$ teraflops. For a two-dimensional problem, this direct propagation of $J_{1,2}$ is not worthwhile.

GPGPU programming - General-Purpose computation on Graphics Processing Units is a technique to use massive parallel computation capabilities of graphics cards for physics simulations. Since the pixels in the ROI can be calculated independently, this problem greatly benefits from massive parallelisation offered by such graphics cards. A speed-up by a factor of approximately 250 has been achieved without optimisation of memory layout.

\subsubsection{Analytic function for $\mathrm{x}$-ray waveguides}

X-ray waveguides (WGs) are small holes in a cladding material; typical state-of-the-art diameters are a few ten nanometres, while the length can be up to ten millimetres [29-35]. Only a discrete set of lateral modes can propagate, while radiative modes suffer strong absorption. But this takes some time, or length, and thus absorption effects play a crucial role in terms of efficiency. The game is: "absorb as much incoherent, but as little coherent energy, as possible." 
The typical set-up of WG based coherent diffraction or propagation imaging is illustrated in figure 2.4. The high-flux KB focus** exhibits limited coherence; a WG placed in the focal plane enhances the degree of coherence. This will be shown by analytical and numerical propagation in chapter 5 , using methods prepared in the current chapter. In figure 2.4 (e) and $(f)$ the experimental far-field of the KB mirror pair and two crossed one-dimensional WGs are shown. While the KB beam shows a lot of stripes and impurity, the WG filtered $\mathrm{X}$-ray beam is much cleaner.

Discrete modes Waveguides have a critical guiding layer thickness $W_{c}$ depending on the cladding material. In the case of a silicon WG, this critical width is nearly $20 \mathrm{~nm}$. A silicon WG with a guiding layer of less than $19.96 \mathrm{~nm}$ supports only one lateral mode; for each $20 \mathrm{~nm}$ it is broadened, one additional mode can propagate [31]. So one can expect a similar behaviour of the degree of coherence as in the case of multi-model lasers. A fraction of the guided modes' energy resides as an evanescent wave inside the cladding and is subject to absorption.

Analytical modes For "perfect" waveguides ${ }^{\dagger \dagger}$, there exist even and odd modes $\psi_{n}$ with respect to the optical axis. The electrical field in the guiding layer is given as either a cosine or a sine of the position, while in the cladding it is exponentially decaying (evanescent wave). Boundary conditions at the guiding layer-cladding-interface give the discrete spectrum and the constants $k, \kappa$ are determined by a transcendental equation [38]. In the following, $D$ denotes the guiding layer thickness, $\rho$ is proportional to the electron density, $m$ is an eigenvalue of the Helmholtz equation and $N$ is a normalisation constant. See figure 2.5 for an illustration of the modes in a particular WG.

$$
\begin{aligned}
& \psi_{n}(y)=N_{n}\left\{\begin{array}{lll}
\cos \left(\kappa_{n} y\right) & , & |y|<\frac{D}{2} \\
+A_{n} e^{-k_{n}|y|} & , \quad|y|>\frac{D}{2} & n \text { even }, \\
\sin \left(\kappa_{n} y\right) & , \quad|y|<\frac{D}{2} \\
\pm A_{n} e^{-k_{n}|y|} & , \quad|y|>\frac{D}{2}
\end{array} \quad n\right. \text { odd } \\
& k_{n}=\sqrt{m_{n}}, \quad \kappa_{n}=\sqrt{\rho-m_{n}} ; \\
& k_{n}=\left\{\begin{array}{ll}
+\kappa_{n} \tan \left(\kappa_{n} D / 2\right), & n \text { even, } \\
-\kappa_{n} \cot \left(\kappa_{n} D / 2\right), & n \text { odd } ;
\end{array} \quad A_{n}= \begin{cases}\cos \left(\kappa_{n} D / 2\right) e^{k_{n} D / 2}, & n \text { even }, \\
\sin \left(\kappa_{n} D / 2\right) e^{k_{n} D / 2}, & n \text { odd } .\end{cases} \right.
\end{aligned}
$$

**The Kirkpatrick-Baez mirror geometry is introduced in chapter 3.

†'"Perfect" means with sharp boundaries, the index of refraction given by a rectangular profile. 
(a) KB based propagation imaging

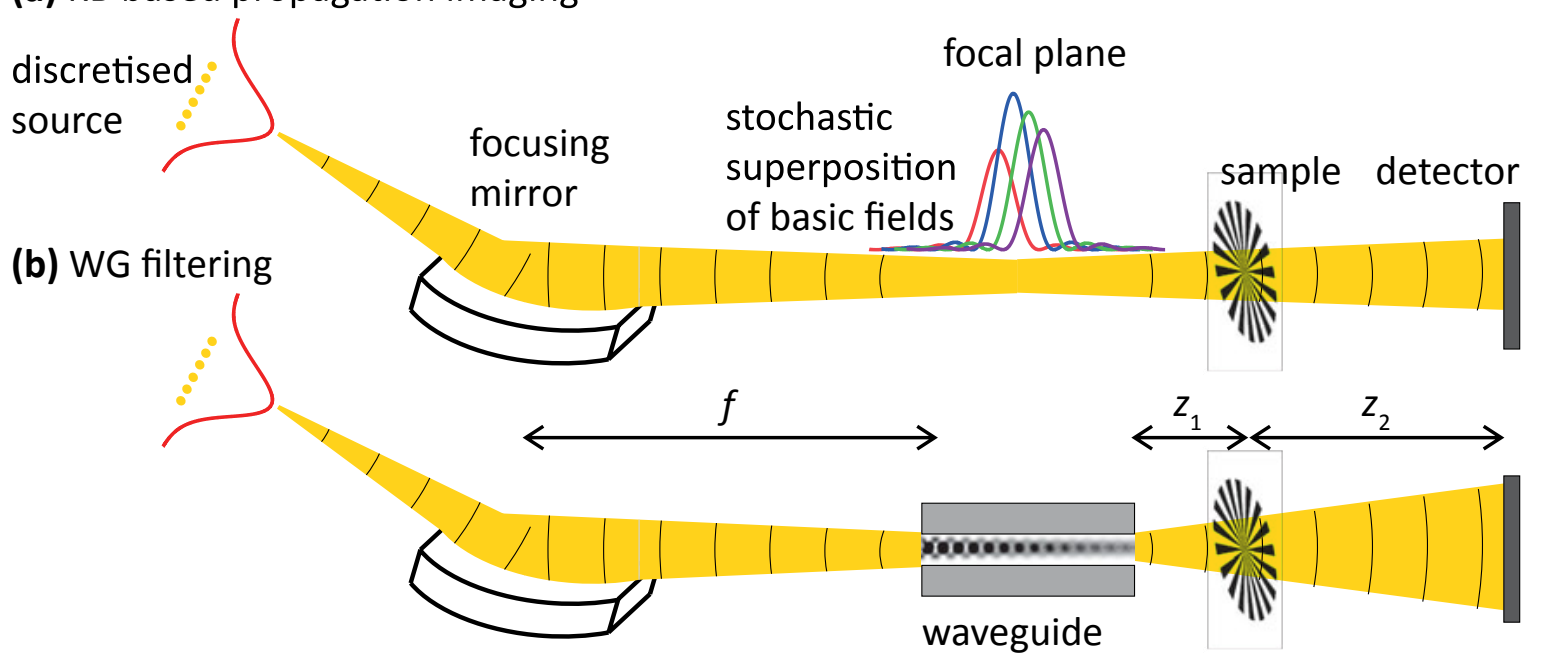

(c) One realization of mirror focus

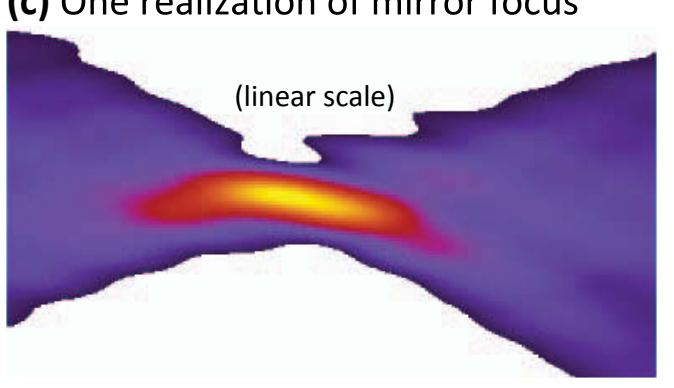

(e) Experimental KB far-field

(d) One realization of WG filtering
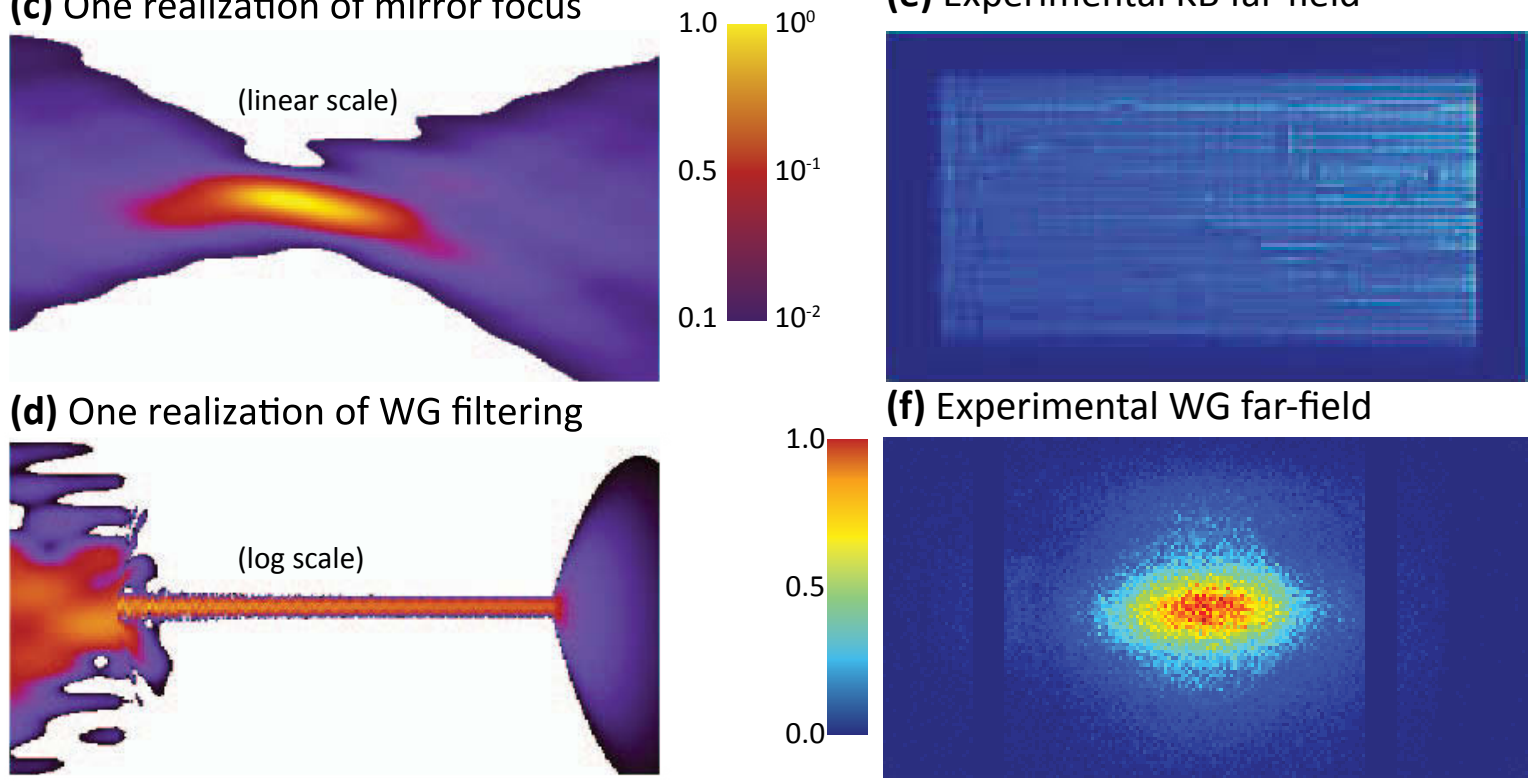

(f) Experimental WG far-field

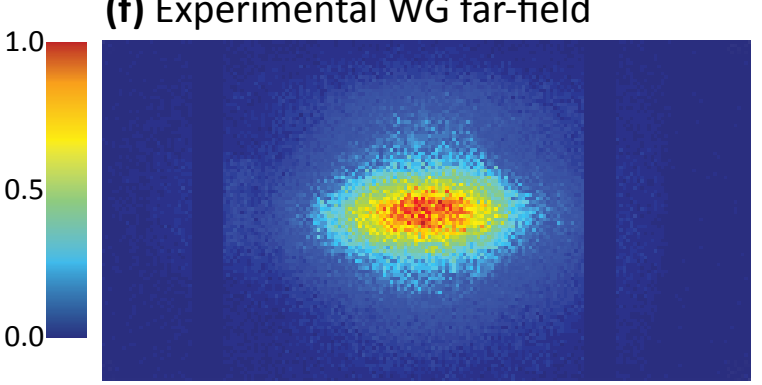

Figure 2.4: The typical set-up of x-ray propagation imaging, using a simple KB mirror (a) or an x-ray waveguide (b) for filtering. In ( $c$, linear colour map representing intensity), one realisation of the stochastic KB focus is shown, while (d, logarithmic colour map) presents the propagation through a one-dimensional WG. In (e) and (f), experimental far-fields of the KB and WG, obtained at the P10 coherence beamline at PETRA III, are shown. The WG not only enhances the degree of coherence, see chapter 5, but also removes inhomogeneities of the KB beam. On the other hand, photon counting statistics due to low transmittance enlarges measurement time and noise level. More information about this particular KB mirror system is currently prepared in [36]; for information about waveguides, cf. [37]. Shown in ( $f$ ) is the far-field of a two-dimensional channel-WG in silicon cladding, of length $1 \mathrm{~mm}$ and guiding core diameter $200 \mathrm{~nm}$. The two dark stripes are due to the detector (Pilatus). 
(a) Waveguide modes - amplitude

(b) Waveguide modes - intensity

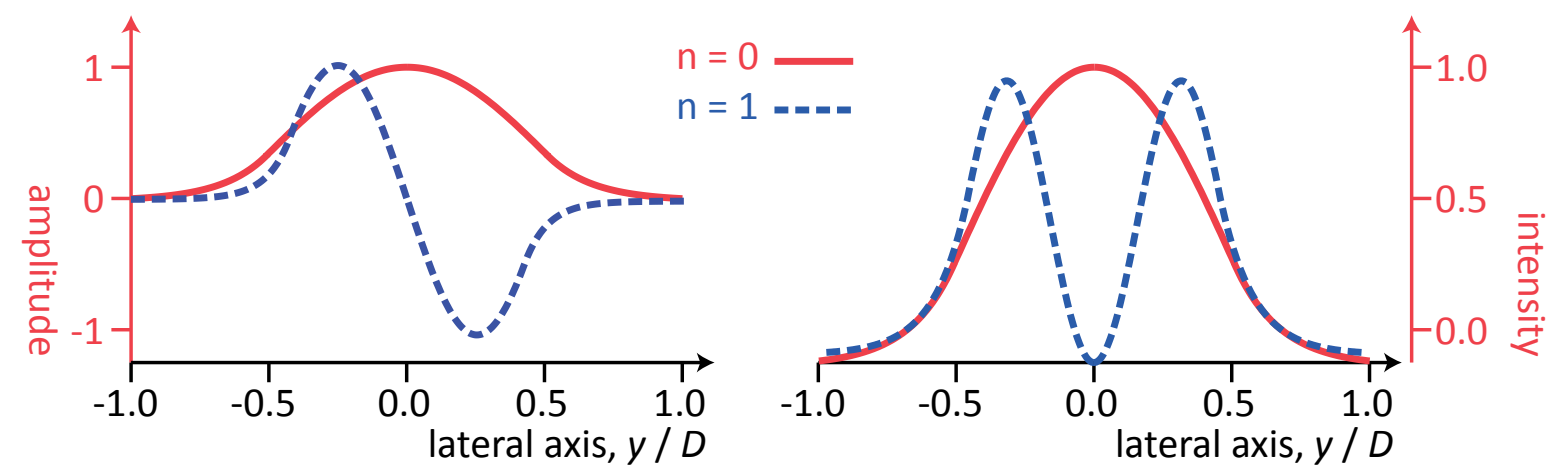

Figure 2.5: Sketch of the first two guided modes in a waveguide. Shown with the red line is the "ground state" mode, while the "first excited mode" is indicated by the blue dashed line. The amplitude (a) can be negative and odd, whilst the intensity (b) is non-negative and even, for our choice of coordinates.

Since the independent modes mix incoherently, one can write the average optical intensity inside the waveguide as

$$
I=\sum \lambda_{n} \psi_{n}^{*} \psi_{n}
$$

where the $\lambda_{n}$ are the occupation numbers of the corresponding modes. More generally we are interested in the mutual intensity

$$
J_{n}\left(x, y_{1}, y_{2}\right)=\lambda_{n}(x) \psi_{n}^{*}\left(y_{1}\right) \psi_{n}\left(y_{2}\right)
$$

We will explore further properties of this analytical model for coherence inside x-ray waveguides in chapter 5 and compare it to numerically obtained data. Up until now, we have not given a method for calculating the occupation numbers $\lambda_{n}$. This is postponed to chapter 5 .

\subsection{Stochastic phase}

In the previous sections we have defined the degree of coherence and mutual intensity of optical fields; let us now take a closer look at the phase in partially coherent fields. If phase relations between point-sources fluctuate with time, the propagated phase is also a random process. Considering a relative phase with respect to a reference point (we will use the focus), the time-harmonic phase $\exp (i \omega t)$ and a "random global phase" due to the random superposition is not of concern here. 
We define the stochastic phase $\phi(x)$ as

$$
\phi(x)=\arg \left(\sum w_{n} c_{n}^{\text {rand }} u_{n}(x)\right) .
$$

The relative phase $\phi_{r}(x)$ with respect to the focus point $F$ is

$$
\phi_{r}(x)=\arg \left(\sum w_{n} c_{n}^{\text {rand }} u_{n}(x)\right)-\phi(F),
$$

where the reference phase $\phi(F)$ is to be calculated by the same tuple of random coefficients. Now we define the average relative phase $\varphi(x)$ in the stochastic ensemble as

$$
\varphi(x)=\left\langle\phi_{r}(x)\right\rangle=\left\langle\arg \left(\sum w_{n} c_{n}^{\text {rand }} u_{n}(x)\right)-\phi(F)\right\rangle .
$$

The relative phase $\varphi(x)$ itself constitutes a random process; its spread is connected to the degree of coherence. In case of a rather coherent illumination one expects a tight distribution of $\phi_{r}$ around $\varphi$, while rather incoherent illuminations yield a widespread distribution. In other words, the "phase" in an incoherent beam is undefined on reasonable observation time scales. Since we are simply adding intensities in that case, the phase is of no concern; actually the word "phase" does not even occur in this description. Partial coherence preserves the phase to some extend. As a quantitative measure we use the root-mean-square $\Delta \varphi(x)$ of the fluctuating (relative) phase $\phi_{r}(x)$ in the stochastic ensemble:

$$
\Delta \varphi(x)=\sqrt{\left\langle\phi_{r}(x)^{2}\right\rangle-\left\langle\phi_{r}(x)\right\rangle^{2}} .
$$

In evaluating $\phi_{r}(x)$, one has to keep in mind that phases are periodic in $2 \pi$. The used computer codes have a domain of $[-\pi, \pi]$. This leads to "phase jumps" that would render the rms-value useless. Instead, "unwrapped" values have to be used; this is possible by scanning the optical axis for such phase jumps, and correcting sequential values by the number of detected jumps (possibly negative), multiplied by $2 \pi$. Care has to be taken to sample with at least the Nyquist frequency to avoid aliasing.

Using this concept, a partially coherent generalisation of the Gouy phase shift will be studied in subsection 3.4.3.

A numerical scheme to simulate propagation of spatial coherence based on stochastic pre-calculated fields has been proposed. This scheme will be used in the following chapters to simulate focused fields for extended sources. A comparison with analytical expressions given for x-ray waveguides follows in section 5.3. 


\section{Chapter 3}

\section{Simple Mirrors}

\subsection{Introduction}

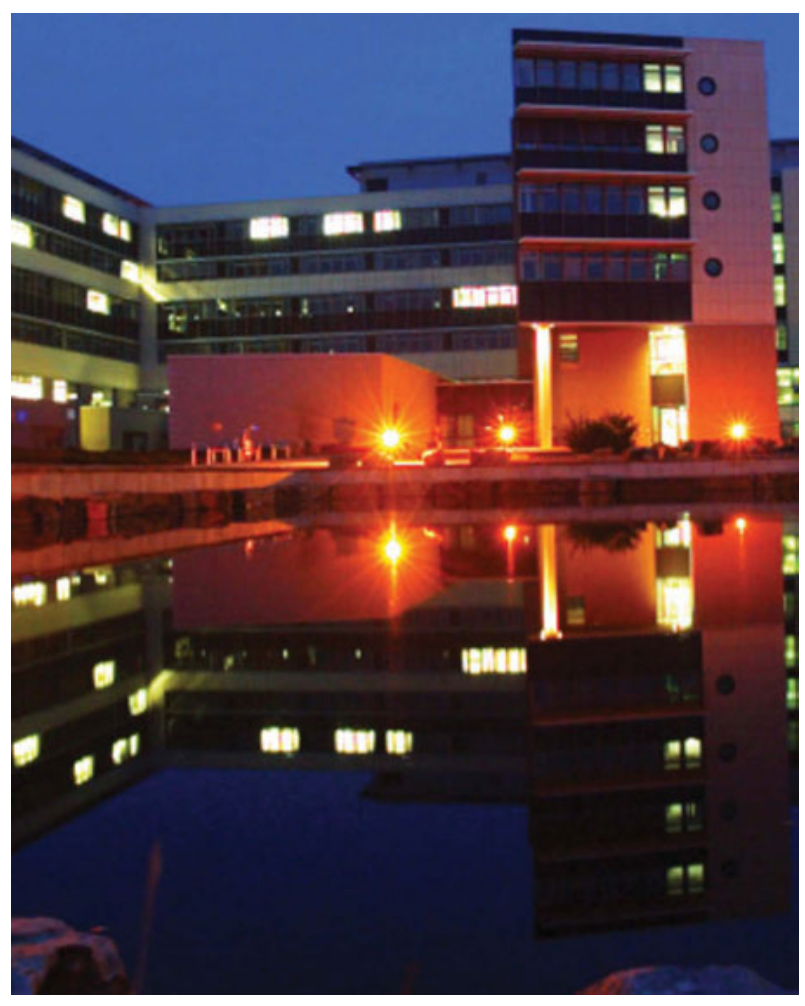

Beam shaping optics are key components in x-ray experiments. Apart from monochromators or simple collimating tools like slits, focusing devices are a helpful, often necessary part of the set-up. In direct imaging they are used as illumination and objective lenses to form the image, while in coherent (or "indirect") imaging they increase numerical aperture and hence resolution compared to the nearly parallel synchrotron beam. Sometimes focusing is just used to enhance flux density or to illuminate only part of the sample.

Depending on the experimental needs (bandwidth, photon energy, efficiency, coherence properties), scientists nowadays can choose between different focusing devices for their $x$ ray experiment. Apart from collimators and $x$-ray waveguides which confine $x$-rays, basically three optical concepts are used for focusing: refraction (lenses), diffraction (Fresnel zone plates), and reflection (mirrors). This chapter is devoted to the latter; multilayer mirrors are described and modelled in chapter 4 . Chapter 5 deals with $\mathrm{X}$-ray waveguides used as coherence filters.

Refraction. In visible optics, lenses are the primary device both for focusing and imaging. But in the x-ray regime where the index of refraction is close to unity, focal lengths of up to hundred metres made the use of lenses impractical. Some twenty years ago it was realised that sequential arrays of, say hundred lenses in a row, along with the corresponding fabrication technology can lead to reduced focal lengths well adapted to the needed demagnification $[39,40]$. In the beginning, cylindrical holes of diameters in the sub-millimetre 


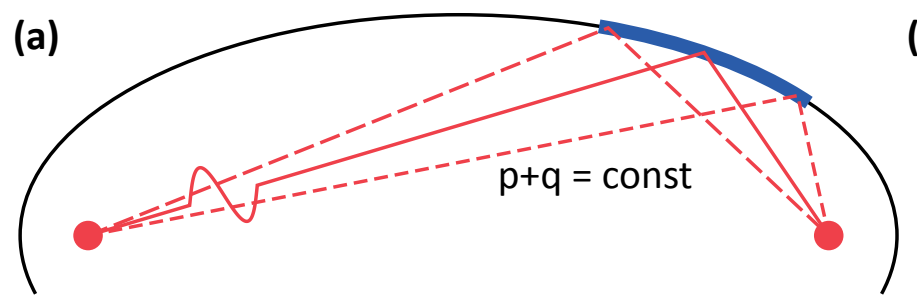

(b)

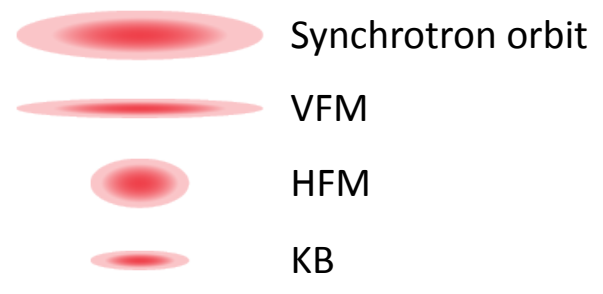

Figure 3.1: Typical set-up of elliptical mirrors used at synchrotron radiation sources for focusing. In (a), the one-dimensional focusing principle based on the elliptical shape is illustrated; in (b) the stages of Kirkpatrick-Baez (KB-)focusing of independent vertical and horizontal focusing mirrors (VFM, HFM) are illustrated. The effective electron distribution in a synchrotron often is approximated by an ellipse around the orbit with an excentricity of about 0.1 ; the diverging $x$-ray beam can be focused in one dimension by an elliptically shaped mirror. In using the KB geometry of two sequential mirrors, decoupling of the two dimensions is assumed.

range were drilled; with improved fabrication techniques including nano-lithography, better layouts to overcome aberration are being used. Computer simulations of 1166 "adiabatic" lenses in a row promise ultra-short focal distances and small spot sizes [40]. Assembling modules allowing to change the number of lenses "online", so-called "transfocators", have been built that allow for "zooming", i.e. customizing the focal length [41].

Diffraction. Focusing optics based on diffraction make use of quasi-periodic structures to scatter the incoming wave in such a way these scattered waves interfere constructively in the focus. A typical example are Fresnel zone plates [42]; sometimes, multilayer Laue lenses [43-45] are also sorted into this category. Multilayer mirrors $[46,47]$ can be considered diffractive, too - but usually they are called reflective optics. We will see in chapter 4 that constructive interference is important.

Reflection. The topic of the current chapter is elliptically shaped (total) reflective mirrors. Since the distance from source point $S$ via the surface to the focal point $F$ is constant, a spherical wave emerging from $S$ interferes constructively in $F$, see figure 3.1 (a). For highest intensity, total reflection mirrors are being used. As critical angle of total reflection and hence numerical aperture are very small, multilayer mirrors with reflectivity at higher angles of gracing incidence are often used.

Apart from special set-ups, two-dimensional focusing is needed. At first sight, this requires mirrors of three-dimensional, ellipsoidal shape. In the year 1948, Paul Kirkpatrick and Albert $\checkmark$. Baez proposed a sequential set-up of two elliptical mirrors, each focusing in a different direction [48]. The assumption of this set-up is that the two dimensions (usually vertical and horizontal) decouple and the system can be described by a tensor product; see figure 3.1 (b). Since a typical synchrotron source shows an elliptical source size with aspect ratios 


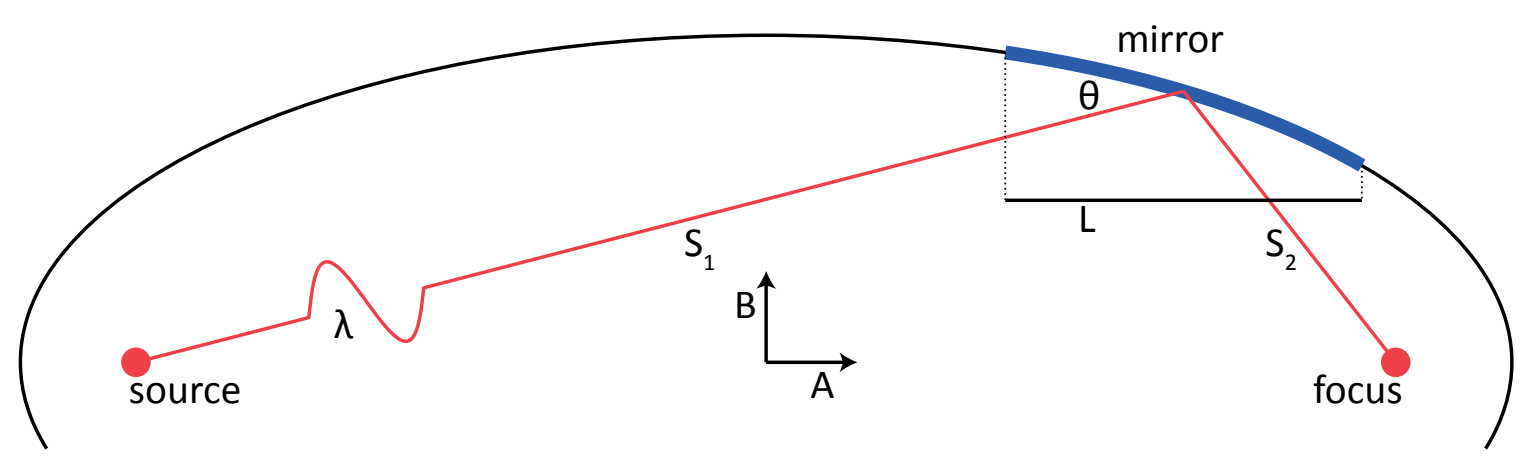

Figure 3.2: Definition of coordinates and parameters for focusing mirrors. The geometry is determined by the distance source-centre of the mirror $S_{1}$, the distance centre of mirror-focus $S_{2}$, and the angle of incidence at the mirror's centre, $\theta$ (measured against the surface). Furthermore, the mirror has a length $L$, here measured projected on the semi major-axis $A$ of the ellipse. The illumination is assumed to be monochromatic with a wavelength $\lambda$.

of 0.2 or smaller, different focal distances - but a common focal plane - offer the possibility of a (nearly) circular focus. In this case, the divergence of the beam is case different in the two dimensions, and the far-field is rectangular.

Advances in fabrication and polishing allow for shapes very close to the desired elliptical profile. With elastic emission machining (EEM) [49] and other polishing techniques, roughness of less than $0.1 \mathrm{~nm}$ and figure errors (i.e., deviation from the ideal profile) well below $1 \mathrm{~nm}$ are achievable. Anyway, real structure effects are important to deal with, both in planning of an optical set-up and in analysis of images. Some of the presented simulation results have been obtained with model or real (measured) deviation profiles taken into account.

Since $x$-rays' index of refraction $n=1-\delta+i \beta$ is smaller than unity, (external) total reflection occurs. For example, the index of refraction of bulk silicon at a photon energy of $E=12 \mathrm{keV}$ is $n=1-3.164 \times 10^{-6}+3.134 \times 10^{-8} i$. The critical gracing angle of reflection is $2.5 \mathrm{mrad}$; the evanescent wave reduces reflectivity to about 96 per cent at an angle of $2.2 \mathrm{mrad}$. In addition, the evanescent wave effectively yields a complex coefficient, making the reflected wave suffer of phase-shift depending on angle of incidence [50]. This phenomenon is known from visual optics as the Goos-von Hänchen effect [51] and will be discussed in subsection 3.2.2.

For a definition of coordinates and parameters used throughout this chapter and the accompanying simulation programme, see figure 3.2 .

In the next section we will briefly address Fresnel's coefficients, the Goos-von Hänchen effect and the Fresnel-Kirchhoff integral of diffraction. We continue with the Gouy phase shift of focusing optics and a brief introduction of the simulation programme. More details of the implementation can be found in appendix C. In section 3.3, results of partially coherent 
focus simulations (see chapter 2) are presented for extended sources, including a discussion of figure errors. Section 3.4 adds results obtained for the defocus region.

A similar simulation programme has been written by Cameron Kewish [50]. Software to predict performance of focusing devices is common at synchrotron radiation facilities, for example SHADOW, IMD, and XOP. Some of these tools rely on geometrical optics and (phase-)ray-tracing; none allows for easy integration of wave-optical theory for curved multilayer mirrors. IMD [52] uses a fast analytical approach based on the Parratt algorithm [8], suitable for flat and infinitely long multilayers. Our new tool, x-ray multilayer mirror simulations (XMLS), is capable of wave-optical simulations of mirrors and multilayer mirrors (see chapter 4). In combination, XMLS and the approach of stochastic optical fields from chapter 2 allow for partially coherent beam x-ray focusing with typical CPU run-times on the order of minutes or hours (depending on number of pixels in region of interest and Monte Carlo noise).

In this chapter we will use several abbreviations and definitions. P10 is the coherence beamline at the PETRA III synchrotron (Positronen-Elektronen-Ringanlage), part of HASYLAB (Hamburger Synchrotronstrahlungslabor) at DESY (Deutsches Elektronensynchrotron) in Hamburg, Germany. The Institut für Röntgenphysik operates an own set-up dedicated to propagation imaging and waveguide-based imaging (see chapter 5), which is composed of two elliptically curved mirrors in Kirkpatrick-Baez geometry. These mirrors will be labelled P10-HFM (for the horizontally focusing mirror) and P10-VFM (vertically focusing mirror). Their geometrical parameters are summarised in table 3.1; for much more details of this set-up, the reader is referred to [12,53], and [36]. Despite state of the art polishing, the mirrors show small deviations from the perfect ellipse, as shown in figure 3.5. Simulations using measured profiles are marked with HFMr and VFMr, while the calculations with the "ideal" elliptical shape are marked with HFMi and VFMi.

\subsection{Theory}

In this section we remind ourselves of necessary theoretical methods and equations to simulate (total) reflection mirrors. We start with a brief reminder of Fresnel's coefficients of reflection, formulated for gracing angles denoted $\theta$; in most textbooks, they are given for the "optical" angles $\vartheta=\pi / 2-\theta$. Subsection 3.2.2 explains the Goos-von Hänchen effect as a result of phase gradients due to evanescent waves. Subsection 3.2.3 introduces the integral of Fresnel and Kirchhoff in "1+1 dimension" for free-space propagation from the mirror's surface to some region of interest. In subsection 3.2.4 the Gouy phase shift present in the defocus region is described. Afterwards, the simulation programme is briefly described; more implementation details can be found in appendix C. The theory follows closely [50]. 


\subsubsection{Fresnel's coefficients in the x-ray regime}

Classical optics are described by the well-known Maxwell equations. The most important solution in vacuum is the set of plane waves. These are fully defined by their wave vector $\vec{k}$ and the dispersion relation $k=\omega / c$. In isotropic, homogeneous media, this is generalised using the index of refraction to $k=\omega / n(\omega) c$.

In x-ray optics, the index of refraction $n=1-\delta+i \beta$ is a complex number; its real part $1-\delta$ is slightly smaller than unity. By this, the phase velocity is greater than the speed of light. N.B., however, that information is transmitted with the group velocity, defined as $\mathrm{d} k / \mathrm{d} \omega$ [8]; this turns out to be less than $c$. Due to the imaginary part $\beta$, waves in media suffer absorption, hence loss of energy and intensity. Often the linear absorption coefficient $\mu=2 k \beta$ is used to describe absorption; the intensity after propagation of a distance $d$ in such a medium is reduced by a factor of $e^{-d \mu}$.

At interfaces of media with different $n_{1,2}$, beams are reflected and refracted. The refracted angles $\theta_{2}$ (measured against surface, see figure 3.3) or $\vartheta_{2}$ (measured against surface normal) are given by Snell's law,

$$
\vartheta_{2}=\sin ^{-1}\left(n_{1} / n_{2} \sin \vartheta_{1}\right), \quad \theta_{2}=\cos ^{-1}\left(n_{1} / n_{2} \cos \theta_{1}\right) .
$$

The reflected amplitude depends on polarisation; Fresnel's coefficient for the $\sigma$-component (component perpendicular to the plane of incidence) of the amplitude is

$$
r_{\sigma}=-\frac{\sin \left(\vartheta_{1}-\vartheta_{2}\right)}{\sin \left(\vartheta_{1}+\vartheta_{2}\right)}=\frac{\sin \left(\theta_{1}-\theta_{2}\right)}{\sin \left(\theta_{1}+\theta_{2}\right)}
$$

while the $\pi$-component (parallel component) is

$$
r_{\pi}=\frac{\tan \left(\vartheta_{1}-\vartheta_{2}\right)}{\tan \left(\vartheta_{1}+\vartheta_{2}\right)}=\frac{\tan \left(\theta_{1}-\theta_{2}\right)}{\tan \left(\theta_{1}+\theta_{2}\right)}
$$

The reflected intensities are given as $|r|^{2}$. Note that under the condition of total reflection (where due to $\beta>0$ the reflectivity is not strictly 1.0), a phase jump of

$$
\Delta \phi=2 \tan ^{-1}\left(\frac{-\sqrt{2 \delta-\sin ^{2} \theta_{1}}}{\sin \theta_{1}}\right)
$$

occurs $[8,23,50,54]$. Using complex numbers in calculating $r$, this phase shift is automatically accounted for. In the following, we will drop the index and call $\theta:=\theta_{1}$ the gracing angle of incidence. The phase shift determines the structure of standing waves [55]. 


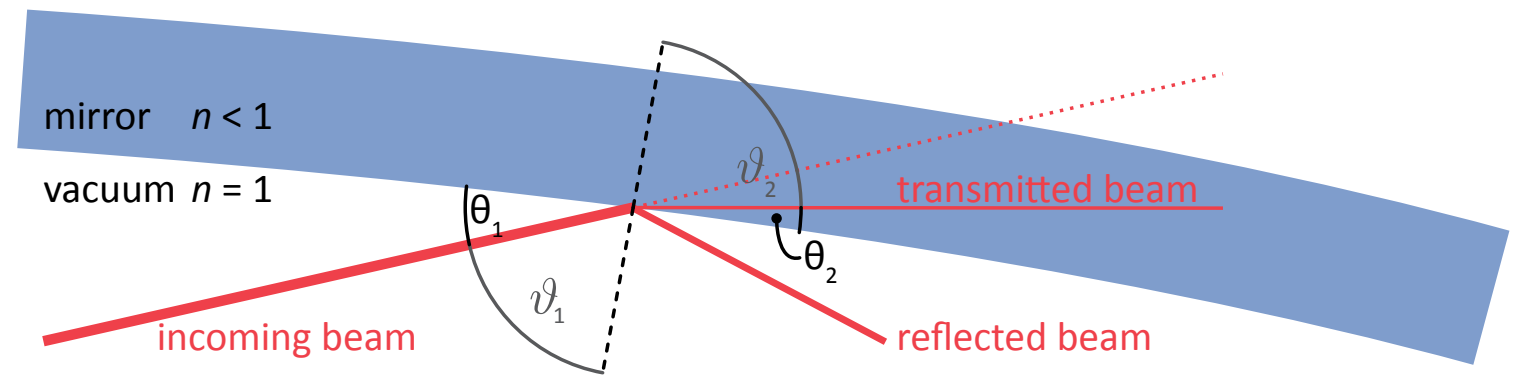

Figure 3.3: Definition of "optical" and "x-ray optical" angles, $\vartheta$ and $\theta$. In x-ray optics, the real part of the index of refraction is usually smaller than unity, $|n|=1-\delta<1$, with $\delta \sim 3 \times 10^{-6}$ for silicon at a photon energy of $E=12.4 \mathrm{keV}$. This means that a transmitted ray is refracted towards the surface; for small angles, $\theta \lesssim 2.5 \mathrm{mrad}$, total reflection occurs.

\subsubsection{Goos-von Hänchen effect}

The aforementioned phase shift $\Delta \phi(\theta)$ depends on the angle of incidence $\theta$ and approaches zero at the critical angle. Since the local angle of incidence, $\theta(s)$, changes along the mirror's surface $s$, a phase gradient is introduced. In a thin-lens approximation, a constant phase gradient in the mirror's plane corresponds to a lateral shift in the focal plane (which can be seen as the Fourier plane in first approximation).

This shift of the focus can be interpreted as a Goos-von Hänchen effect known from experiments in the visible regime of optics: Beams impinging at total reflection at a boundary seem to be reflected at a laterally shifted point. Goos and von Hänchen measured this shift to be on the scale of a wavelength, hence for an accurate measurement many reflections and a very confined beam had to be used in their experiment in $1943[51,56]$.

In terms of wave optics, the phase shift $\Delta \phi$ is attributed to the evanescent wave: Although totally reflected, the wave penetrates the second medium, but exponentially decaying. The evanescent wave propagates parallel to the surface, accumulating phase due to $n \approx 1-\delta$. The evanescent wave is the optical analogue of quantum mechanic's tunnel effect.

In figure $3.4(\mathrm{a})$, both the local angle of incidence $\theta(s)$ (blue line) and the resulting phaseshift $\Delta \phi(\theta)$ (green dashed line) are shown as a function of the surface coordinate $s$ for the P10-HFM mirror*. At $\lambda=0.1 \mathrm{~nm}$, the average phase gradient of $-0.49 \mathrm{rad} / 320 \mu \mathrm{m}$ along the projected aperture corresponds to a deflection angle of $24.4 \mathrm{nrad}$, hence the focus point should be displaced by $\Delta y \approx-4.88 \mathrm{~nm}$, as estimated by a simple formula:

$$
\Delta y \approx f \times \alpha=f \times \frac{\partial \phi \cdot \lambda}{\partial(L \theta) \cdot 2 \pi}
$$

*See table 3.1 for geometrical and physical parameters. 
(a) Local angle and phase shift

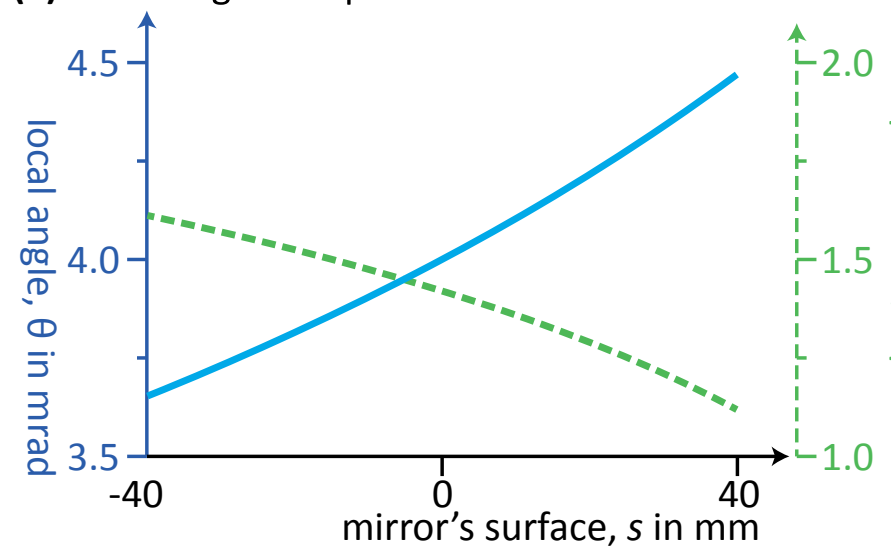

(b) Simulated focus

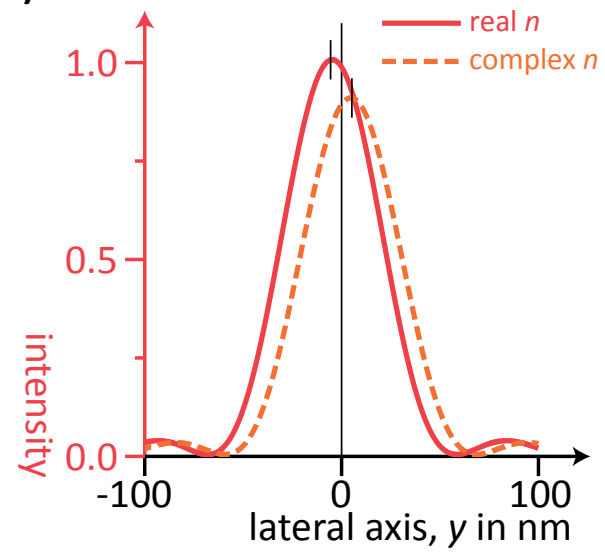

Figure 3.4: Goos-von Hänchen shift, simulated for the P10-HFM. In (a), local angle $\theta(s)$ (blue line) and phase shift (green dashed line) along the surface are shown, while in (b) the intensity in the focal plane has been calculated. The red line is a simulation result using only the real part of the index of refraction, $n=1-\delta$, while the orange dashed line has been calculated with $n=1-\delta+i \beta$.

with focus length $f$, wavelength $\lambda$, and phase shift gradient $\partial \phi / \partial(L \theta)$, were $L$ denotes mirror length and $\theta$ the average angle of incidence. Numerical propagation yields a shift of $-4.78 \mathrm{~nm}$, see figure 3.4 (b). The red line has been simulated for a Pd mirror, using the respective real valued index of refraction. For the orange dashed line, the complex valued index of refraction that includes absorption has been used. This yields an additional phase shift of opposite sign, but roughly twice as large. The resulting focus shift is $+4.79 \mathrm{~nm}$, negligible compared to the focus size of $\Delta=54(1) \mathrm{nm}(F W H M)$ in both cases.

The data are consistent with no impact on focus size of $54 \mathrm{~nm}$.

The simple estimation (3.5) assumes a constant propagation distance $f$, whilst the actual distance from the curved mirror to the focus changes. The numerical propagation, on the other hand, accounts both for this effect and $\Delta \phi$ 's dependence on surface position.

\subsubsection{Fresnel-Kirchhoff integral}

Propagation of a complex valued coherent monochromatic optical field in free space can be modelled with the Fresnel-Kirchhoff diffraction integral (FKI) $[54,57]$. For each point in the target region, the amplitudes of the source are multiplied by a spherical wave term and added up; this scheme is an implementation of Huygens' elementary sources. Arnold Sommerfeld discussed subtle mathematical limits and improvements [58].

For convenience propagation in "1+1 dimensions" is used in this thesis, i.e. source and 
target plane are one-dimensional arcs or lines; to propagate from source points $\overrightarrow{r^{\prime}}$ to target points $\vec{r}$, we write

$$
\psi(\vec{r})=\frac{1}{i \lambda} \int \psi\left(\overrightarrow{r^{\prime}}\right) \frac{\exp (i k \Delta \vec{r})}{\sqrt{\Delta \vec{r} L}} \mathrm{~d} A
$$

In this form, an obliquity factor has been dropped, which usually is unity in the geometry of total reflection mirrors; in the simulations, it is included. In our $1+1$ dimensional approach we propagate cylindrical waves; the length $L$ in the denominator represents the height of such a cylinder, whilst $\mathrm{d} A$ integrates over the cylinder barrel.

\subsubsection{Gouy phase shift}

Following a light wave along the optical axis through the focal plane, a phase shift of $\pi / 2$ (for one-dimensional foci, $\pi$ for two-dimensional ones) is accumulated [17]. A Gaussian beam is defined as

$$
\psi(x, y)=\psi_{0} \frac{w_{0}}{w(z)} \exp \left(-r^{2} / w(z)^{2}\right) \exp (-i k r / 2 R(z)) \exp (i \zeta-i k z)
$$

with minimum beam waist $w_{0}$, beam waist $w(z)$ along the optical axis $z$, phase curvature $R(z)$ and wave number $k=2 \pi / \lambda$. This beam exhibits a phase term $e^{i \zeta}, \zeta=\tan ^{-1}\left(z / z_{0}\right)$. Propagating along the optical axis, this evaluates to $-\pi / 2$ at $-\infty$ and $\pi / 2$ at $+\infty$, continuously incrementing with steepest derivative in the focus. In case of fields focused by lenses, a similar phase is obtained, with some oscillatory behaviour due to diffraction effects. Corresponding phenomena will be encountered in our numerical analysis of $\mathrm{x}$-ray reflection mirrors. Although the scalar Gaussian beam is an important model system in optics, and often used to describe i.e. laser light, it does not satisfy the wave equation [59], but the parabolic wave equation.

\subsubsection{The programme}

As a prerequisite for the multilayer simulation code, a modular programme has been written in $\mathrm{C}++$ that models focusing of cylindrical waves emerging from a point-source by a "simple mirror". The mirror itself is described by the Fresnel coefficient of reflection. Propagation is modelled by a numerical summation of the Fresnel-Kirchhoff integral. The computer code is organised in interacting classes that each simulate different parts of the physical set-up, like the source, the mirror and an array of line detectors. Currently, mirrors cannot be "switched" online, the programme has to be recompiled after a simple change to the source code. 
XMS \& XMLS For development and first testing purposes, a programme called XMS for $X$ ray mirror simulations has been written; later it was migrated into a more modularised code base, the $x$-ray multilayer mirror simulations. In the latter, physical objects are modelled as $\mathrm{C}++$-classes. This enables for easy change and adoption of different models. The main user interface (UI) of XMLS is based on the readline library featuring a command line interface for interactive changing of parameters and starting the calculation. For remote access and as a graphical UI, essential functions are available via HTTP (hypertext transportation protocol): simple calculations can easily be controlled via a web browser; more elaborated remote control from other programmes (like Matlab, gnuplot etc.) is possible by directly sending HTTP commands to the XMLS kernel. A detailed reference with example codes can be found in appendix $G$. The basic class design of XMLS was re-used for other projects.

Draft overview of classes Here we give a small overview of the different classes used in XMLS. For details, see appendix C. XMLS is composed of three parts: A "kernel" with its interface classes (CInterface, CIReadline, and CIWebserver) that store the current parameters and can start the simulation. For each simulation run, an instance of type CKernel is created, along with physical and non-physical classes. The former are abstract notions that model different "devices" like a point-source, the mirror, and a detector. Nonphysical classes contribute helper functions for data management, analysis, and plotting.

Techniques: MPI, SSE \& openMP For reasonable performance, different optimisation techniques have been used to numerically evaluate the FKI. In first tests, message passing interface (MPI) has been used to share the computational load amongst a large number of computers. With the advent of multi-core processors, now good performance is available through single computers; thus the MPI approach was abandoned and replaced by openMP to schedule parallelizable computations amongst different CPUs or CPU cores. Additional speed-up could be achieved using Streaming SIMD Extensions (SSE). The illumination of the mirror surface by a distant point-source (typical distances are on the order of tens or even hundreds of metres) has to be calculated with sub-wavelength accuracy; hardware precision of floating point numbers turned out to be unreliable. To overcome this problem, two solutions exist. The critical equations could be replaced by Taylor approximations; but then the validity and number of terms has to be checked for different set-ups. Instead, precision has been raised using the GNU library for multiple precision calculations ${ }^{\S}$. Whilst floating point arithmetic based on double precision numbers uses a mantissa of 52 bit, GMP allows arbitrary lengths. For typical beamline parameters, floating point operations should be carried out with about 100 bit. The current implementation uses 256 bit. Computational

\footnotetext{
†GNU readline: http://www.gnu.org/s/readline/

$\ddagger$ Single instruction, multiple data

$\S$ GMP, see http://gmplib.org.
} 
impact is very low for the affected part of the code. See [60] for a complete and exhaustive discussion of such techniques.

Tests We only give a brief summary of plausibility and analytical tests that have been carried out with XMLS. Most important are basic focus properties: constructive interference in the focal spot, a nearly sinc ${ }^{2}$-pattern fulfilling the diffraction limit. Also the Gouy-phase and Goos-von Hänchen shift could be observed as predicted by analytical theory. See also subsection 3.3.1.

A computer code to simulate one-dimensional elliptically shaped focusing mirrors has been written and tested. In the following sections results will be presented to predict focused x-ray fields. In combination with the scheme from chapter 2 , focusing of extended sources is carried out and compared to experimental data.

\subsection{Partially coherent focus simulations}

Theory and simulation code having been summarised, this section presents our results of partially coherent focus simulations for $x$-ray total reflection mirrors. We start with the focus of a point-source and distortions due to figure errors, before generalizing towards partially coherent sources. Subsection 3.3.4 shows results on how to improve coherence properties with slits in front of the mirror (on the source side). In section 3.4, results for the defocus region, e.g. along the optical axis, are shown. While the simulation code was prepared, the Institut für Röntgenphysik installed a set of KB mirrors for a nano-focus and holography end-station at the coherence beamline P10 at PETRA III in the framework of BMBF projects 05KS7MGA and 05K10MGA. Most of the following results have been obtained for these two mirrors. The parameters of the horizontally focusing mirror (HFM) and vertically focusing mirror (VFM) can be found in table 3.1 and in [53]. A more verbose description of the beamline is also given in [53]. Some of the results shown below have already been published in $[12,46,53,61]$. Figure 3.5 shows measured height deviation profiles of the two mirrors. 


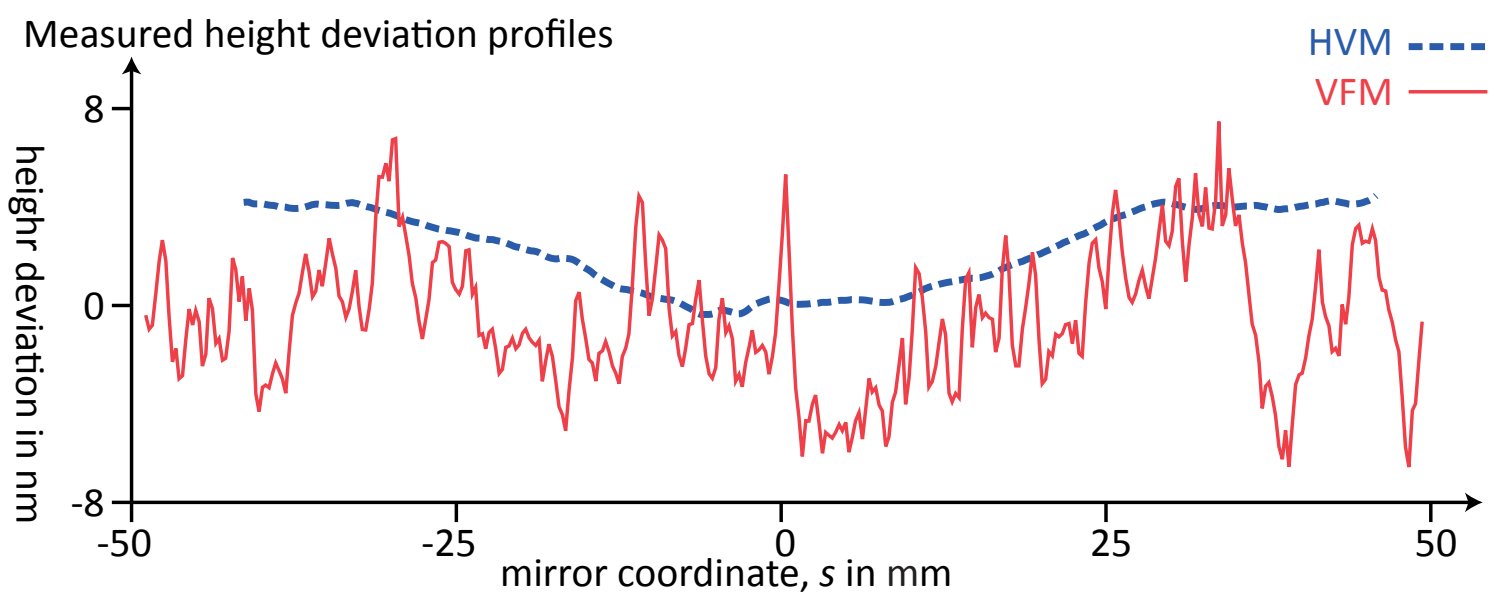

Figure 3.5: Measured figure errors of the HFM and VFM at P10 coherence beamline's nano-focus and holography end-station. Data have been measured by Frank Siewert, BESSY (HFM, blue, dashed line), and WinlightX (VFM, red line), cf. [36, 62].

\begin{tabular}{rrrl} 
Quantity & HFM & VFM & Unit \\
\hline distance from source & 87.7 & 87.6 & metre \\
distance to focus & 200.0 & 305.0 & millimetre \\
angle of incidence & 4.0 & 4.0 & milliradian \\
length & 80.0 & 90.0 & millimetre \\
coating & $\mathrm{Pd}$ & $\mathrm{Pd}$ &
\end{tabular}

Table 3.1: Geometrical parameters of the considered total reflection mirror KB system, part of the nano-focus and holography end-station at the coherence beamline P10 at PETRA III.

\subsubsection{Focus of a point-source}

Most tests of XMLS have been done for a mirror of Fresnel reflectivity, illuminated by a pointsource. This is the simplest set-up. Because the real undulator source has a finite spatial extent and is not perfectly coherent, experimental comparison is not easily possible. The following simulations are valuable as a help in experiment design, particularly for geometrical

\begin{tabular}{rlcl}
\multicolumn{3}{c}{ Focus size } \\
Mirror & point-source & extended source & Coherence length \\
\hline P10-VFM & $84(1) \mathrm{nm}$ & $99(1) \mathrm{nm}$ & $243(13) \mathrm{nm}$ \\
P10-HFM & $54(1) \mathrm{nm}$ & $212(1) \mathrm{nm}$ & $85(2) \mathrm{nm}$
\end{tabular}

Table 3.2: Simulated focus sizes for P10-HFM and P10-VFM, calculated for a photon energy of $12.4 \mathrm{keV}$ and in low beta operation. All values are given as full width at half maximum (FWHM) and are obtained by a Gaussian fit. Uncertainties have been obtained from this fit. Coherence length will be defined further below. 
mirror parameters and surface quality requirements.

The diffraction limited focus properties of the HFM and VFM have been calculated for a photon energy of $E=12.4 \mathrm{keV}$ and are summarised in figure 3.6 (HFM) and in figure 3.7 (VFM). The focus size and coherence length in the focal plane are given in table 3.2. Due to the finite source size and possible misalignments actual focus sizes on the order of $120 \ldots 380 \mathrm{~nm}$ are obtained [45]. In figures 3.6 and 3.7, the left column shows results for the ideal mirror, while for the right column measured height deviation profiles have been included into the simulation. Sub-figure (a) shows the intensity distribution in the focal region; in (b) the phase of the focused $\mathrm{x}$-ray beam is shown. In this data, the harmonic plane wave has been subtracted. Lateral line cuts right in the (best) focal plane are shown in (c).

According to the diffraction limit - see also (4.1) -,

$$
\Delta=\frac{0.88 \lambda}{\mathrm{NA}}
$$

the theoretical lower bound for the spot size depends on the numerical aperture (NA), and thus on several geometrical parameters. Here, $\Delta$ is the full width at half maximum (FWHM) for an assumed sinc $^{2}$-distribution of intensity, which is the case within the thinlens approximation. By scaling the following quantities, the spot size should decrease: photon energy $E$, mirror length $L$, angle of incidence $\theta$; focus length $S_{2}$ (this scales inversely). A numerical simulation allows for more rigorous treatment including the curved geometry and Fresnel reflectivity (limited effective numerical aperture NA). In figure 3.8, the simulated spot size (FWHM) of the ideal HFM is shown as a function of parameter scaling. The reference values are given by the "default geometry" and a photon energy of $12.4 \mathrm{keV}$.

\subsubsection{Influence of figure errors}

The intensity and phase distribution in the focal region of the HFM (figure 3.6) and the VFM (figure 3.7) have been simulated for an illumination of a point-source, both for the ideal mirror (perfect ellipse, left column in the figures) and the real mirror (right column; see figure 3.5 for the measured height deviation profile). In part (a), the intensity distribution is shown on a linear scale. The ideal mirrors show the typical shape expected from analytical and numerical calculations carried out e.g. by Emil Wolf and Edward Hubert Linfoort in the 1950s [63]. They have generalised prior calculations by Zernike and Nijboer in 1949 [64] and included the phase distribution as well. In these impressive and elaborate calculations using Lommel variables [65], the three-dimensional focus of aberration free two-dimensional lenses have been studied; here we deal with one-dimensional reflecting mirrors with gradients both in reflectivity and phase. The one-dimensional problem itself considered here yields slightly 
different results, but agrees well from a qualitative point of view. This is also the case for the phase distribution, shown in part (b).

Precisely polished mirrors have already been simulated and thoroughly analysed elsewhere $[50,66]$. The results presented in this chapter have proven to be of significance in interpreting the measurements at the P10 beamline.

Real structure's influence in case of the superiorly polished HFM (see figure 3.5) is negligible. The "best focal plane" with smallest spot is shifted towards the mirror by about $150 \mu \mathrm{m}$; the focal size itself does not change within the uncertainty of e.g. a sinc ${ }^{2}$-fit; see table 3.2. Figure 3.6 (c) shows a line cut of intensity and phase in the (best) focal plane. In case of the ideal mirror (left column), intensity agrees well with a sinc ${ }^{2}$-function. The phase is rather flat (except for some small angle mismatch with the optical axis and characteristic phase jumps of $\pi$ between side lobes). Including the measured height deviation profile, the central spot is hardly influenced; it is only higher order side lobes that are significantly distorted. The phase distribution gets rather curved, the phase jumps are washed out.

In case of the VFM, height deviations introduce significant distortions of intensity and phase distribution, see figure 3.7 (right column). The best focal plane (defined by highest peak intensity) is shifted by about $800 \mu \mathrm{m}$ towards the mirror; the side lobe structure is changed strongly. The phase distribution is affected significantly.

These simulations have been carried out using the measured height deviation profiles for the two mirrors. A more quantitative study of figure errors' influence on focus sizes would be possible, but is beyond the scope of this thesis.

An extension of the work in view of further upgrades or similar beamline concepts as P10 could address the following issues:

Instead of measured profiles, consider an ensemble of parametrised (like wavelengths, amplitudes, and correlation lengths) of random profiles; simulate the complex amplitude for each profile in the ensemble, and study, for example, the following quantities as a function of the parameters: focus size, focus position along the optical axis, fraction of energy in the side lobes, curvature of the phase front within the central spot, to name a few.

\subsubsection{Focus of extended source}

In this section we present results obtained using the partially coherent propagation scheme introduced in chapter 2 . The spatially extended, assumed quasi-monochromatic, source is modelled by a large number of independent point-sources. These virtual point-sources are 


\section{Focus simulations for a point-source - P10-HFM}

\section{ideal mirror}

(a) Intensity distribution

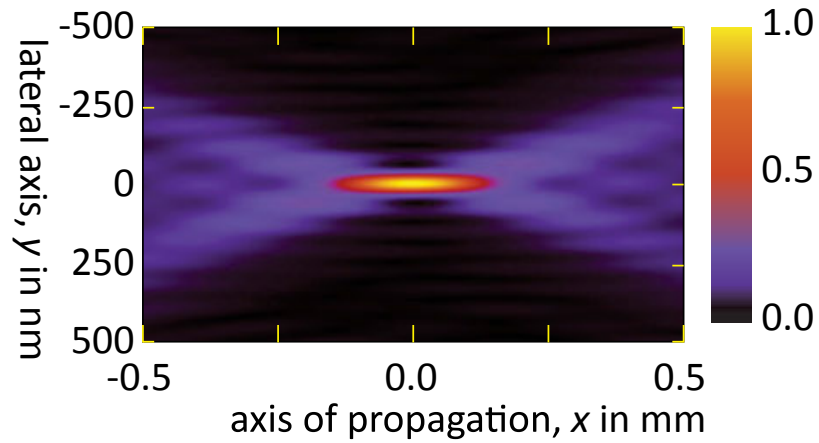

(b) Phase divided by plane wave

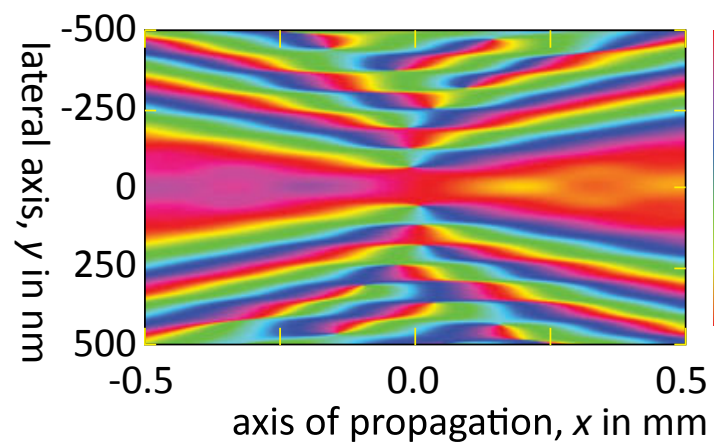

(c) Focus cut

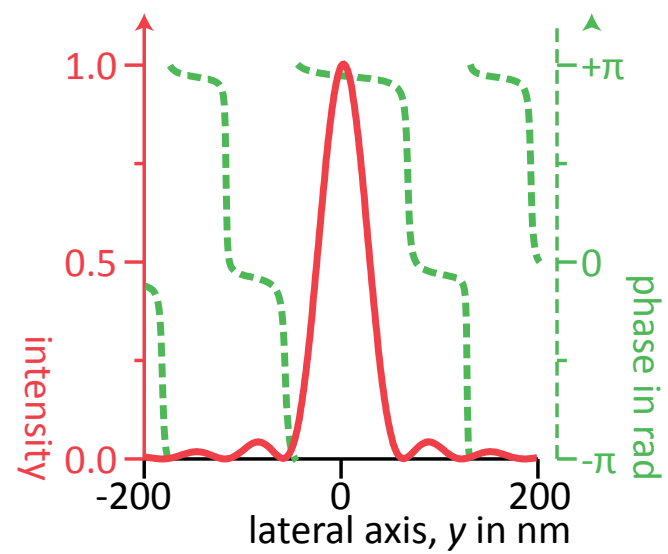

real mirror
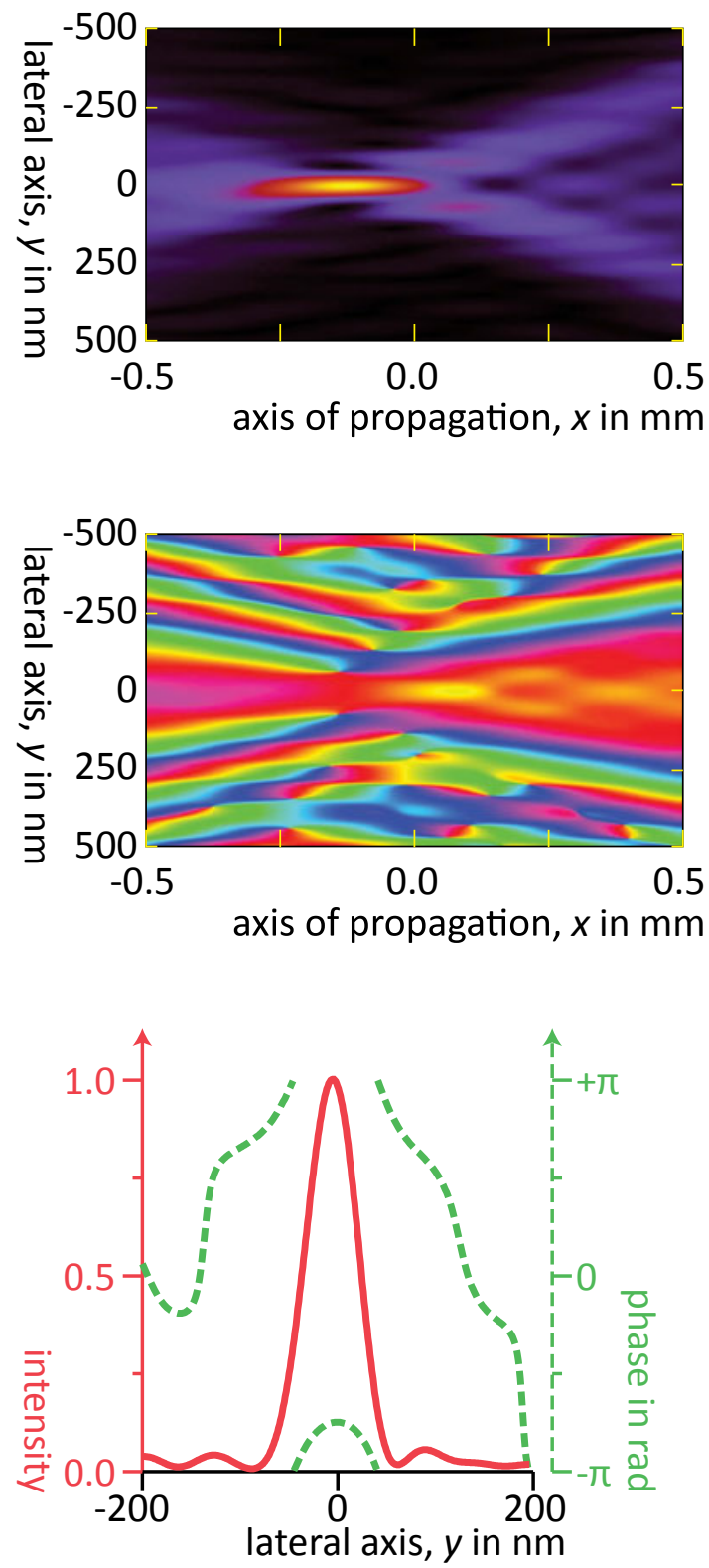

Figure 3.6: Simulation results for a single point-source, here for the P10-HFM. The left column has been obtained for a perfect mirror, while in the calculations shown in the right column, a measured height deviation profile has been included. In (a), the intensity distribution in the focal region is shown, while (b) shows the phase, divided by a plane-wave; a lateral cut of intensity and phase is shown in (c). 


\section{Focus simulations for a point-source - P10-VFM}

\section{ideal mirror}

(a) Intensity distribution

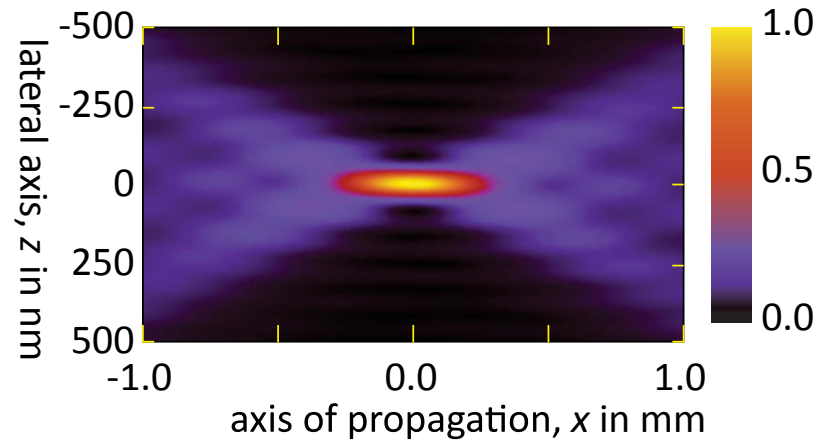

(b) Phase divided by plane wave

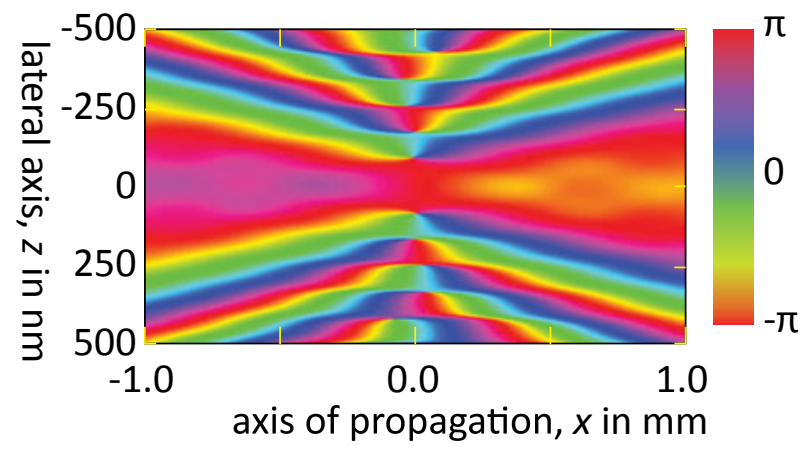

(c) Focus cut

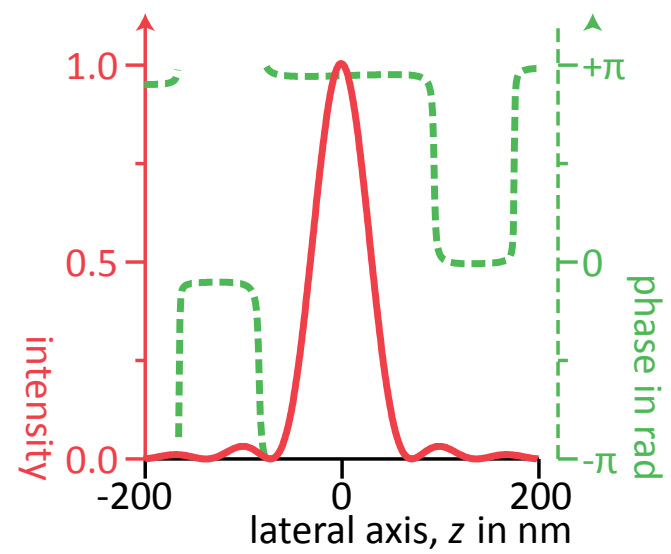

real mirror
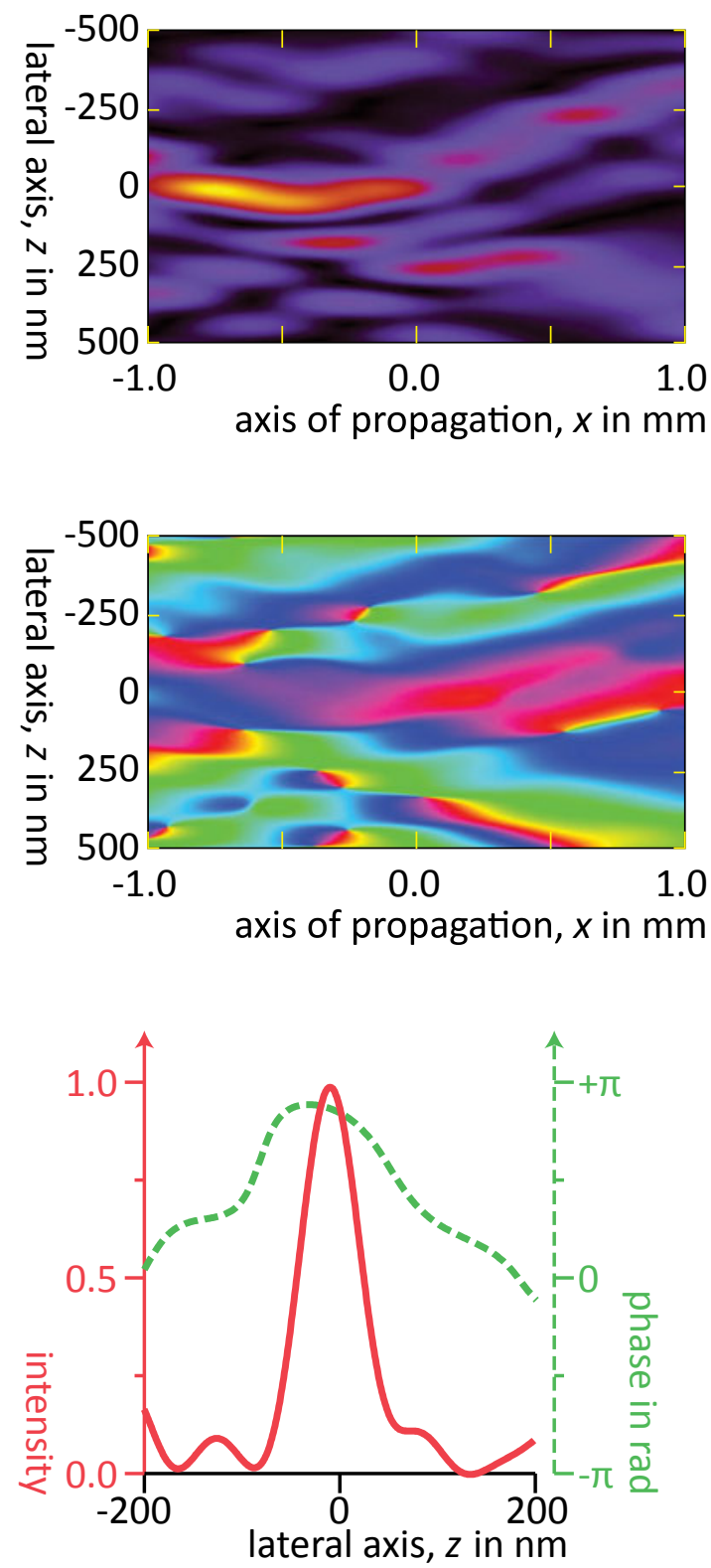

Figure 3.7: Simulation results for a single point-source, here for the P10-VFM. The left column has been obtained for a perfect mirror, while in the calculations shown in the right column, a measured height deviation profile has been included. In (a), the intensity distribution in the focal region is shown, while (b) shows the phase, divided by a plane-wave; a lateral cut of intensity and phase is shown in (c). 
(a) Focus size vs. parameter scaling

(b) Zoom-in

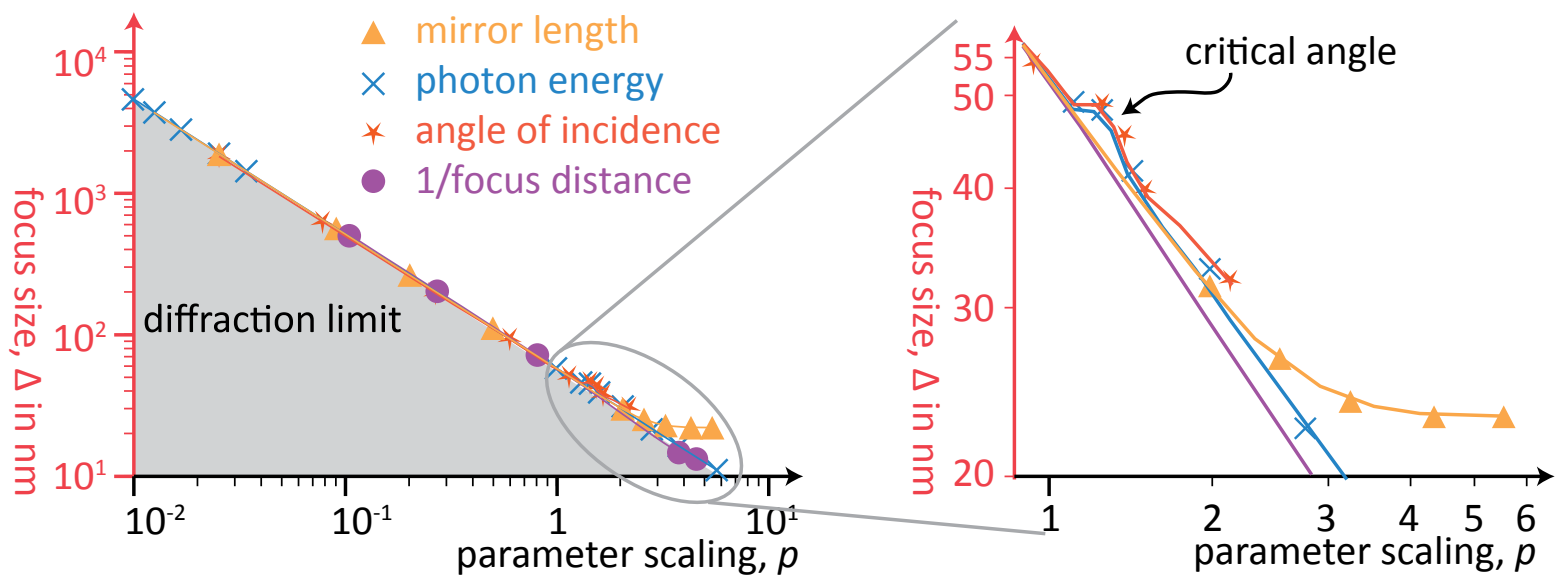

Figure 3.8: Simulated diffraction limit for the ideal P10-HFM as a function of parameter scaling. The abscissa shows a scaling factor, where 1 is the default geometry (at a photon energy of $12.4 \mathrm{keV}$ ). The different curves show the simulated focus sizes for scaling of the indicated parameters.

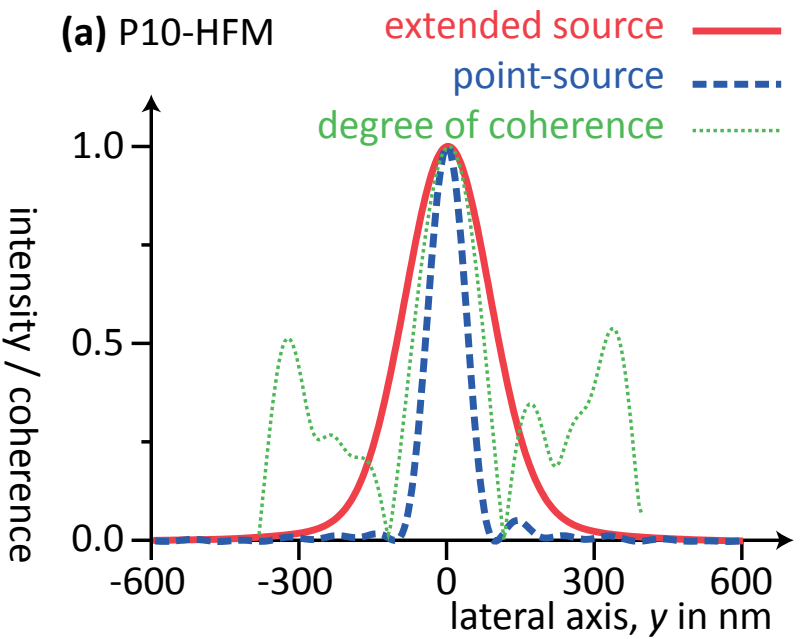

(b) P10-VFM

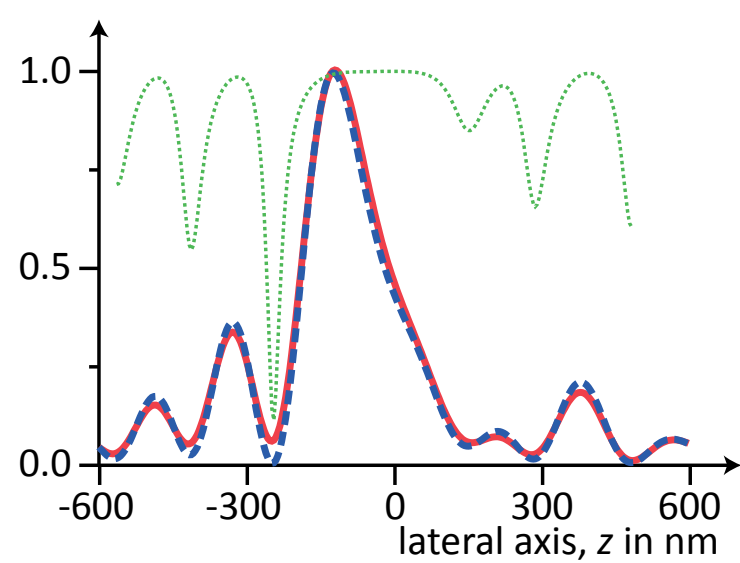

Figure 3.9: Intensity distribution of HFM (left) and VFM (right) at the coherence beamline P10, simulated for a photon energy of $7.9 \mathrm{keV}$. Red lines show partially coherent intensity in low beta mode, whilst blue dashed curves are intensity of the central point-source only. Green dotted lines show degree of coherence with respect to the optical axis. Height deviation (figure 3.5) is respected.

placed along the semi minor-axis of the ellipse and weighted by a Gaussian distribution of width $\sigma$. The beamline can be operated either in low beta mode or high beta mode; in table 3.3, the respective source sizes in both dimensions are given for both operational modes. The data are taken from the technical design report of PETRA III [10].

Note that the actual coherence properties of undulator sources are not only given by the source size, but also affected by the divergence of the beam. Within the Gaussian-Schell 


\begin{tabular}{r|rr|lr|lll} 
operation mode & $\sigma_{\mathrm{v}}$ & $\sigma_{\mathrm{h}}$ & $\Delta_{\mathrm{v}}$ & $\Delta_{\mathrm{h}}$ & $\xi_{1}(\mathrm{v} \times \mathrm{h})$ & $\xi_{2}(\mathrm{v} \times \mathrm{h})$ & unit \\
\hline low beta & 6.0 & 36 & 320 & 2455 & $233 \times 39$ & $250 \times 39$ & $\mu \mathrm{m}$ \\
high beta & 5.5 & 141 & 336 & 702 & $254 \times 10$ & $275 \times 10$ & $\mu \mathrm{m}$
\end{tabular}

Table 3.3: Source and beam sizes at PETRA III, P10 beamline. The values $\sigma_{\mathrm{v}, \mathrm{h}}$ are the root mean square (rms) sizes of the undulator source, in the vertical and horizontal direction, respectively. Beam sizes $\Delta_{\mathrm{V}, \mathrm{h}}$ are calculated for a propagation distance of $87.7 \mathrm{~m}$ and a photon energy of $12 \mathrm{keV}$. Coherence lengths $\xi_{1}$ are calculated for incoherent sources, $\xi_{2}$ account for emittance effects [11].

model (GSM), such sources are described by two quantities: source size $\sigma_{\mathrm{s}}$ and a coherence length in the source plane, $\sigma_{\mathrm{c}}[6]$. In the horizontal direction, usually $\sigma_{\mathrm{c}} \ll \sigma_{\mathrm{s}}$, and the coherence properties are mainly given by the source size. But in the vertical direction, often $\sigma_{\mathrm{c}} \gtrsim \sigma_{\mathrm{s}}$ is achieved [11]. In this thesis, the limit of an incoherent source is assumed.

The following results thus underestimate the vertical coherence properties. In table 3.3, the expected coherence lengths in the mirror plane are calculated. The values $\xi_{1}$ are given in the limit $\sigma_{\mathrm{c}} \rightarrow 0$, while $\xi_{2}$ account for a coherent source given by the GSM and the parameters in [11]. Clearly, $\xi_{1}$ and $\xi_{2}$ agree well for the horizontal direction, while the GSMvalue $\xi_{2}$ gives a slightly longer coherence length in the vertical direction. These values have to be compared with the geometrical acceptance of the KB mirror pair, which is on the order of $320 \mu \mathrm{m}$. Hence the HFM will be illuminated with a partially coherent beam, while a degree of coherence close to unity is expected for the VFM.

Numerical results for the two mirrors are shown in figure 3.9. The simulations have been carried out for a photon energy of $7.9 \mathrm{keV}$ and the nominal source sizes of table 3.3, low beta mode. The geometrical parameters of the set-up can be found in table 3.1, together with a plot of the used height deviation error profiles. The source has been modelled using 601 (HFM) or 401 (VFM) non-correlated point-sources and 10,000 stochastic realisations using the scheme described in chapter 2. The red lines in figure 3.9 show the partially coherent intensity in the best focal plane, while the blue dashed curves show the intensity of the central point-source only. The green dotted lines show the degree of coherence with respect to the optical axis. As expected from the parameters, the HFM produces a partially coherent field, while the VFM focuses nearly fully coherently.

These data have been obtained for the "fully illuminated" mirrors. In the following subsection we will study the influence of slits in front of the KB box.

\subsubsection{Improving coherence with slits}

As specified in table 3.3, the large horizontal source size yields coherence lengths in the mirror plane of $39 \mu \mathrm{m}$ (low beta) and $10 \mu \mathrm{m}$ (high beta, rms values); the HFM's aperture, 
though, accepts $320 \mu \mathrm{m}$. Hence the illumination is only partially coherent, resulting in low contrast for diffraction experiments. The experimental set-up allows for a reduction of the effective aperture: a pair of slits in front of the KB box can be closed to confine the incoming beam width. In this subsection we estimate the coherence properties and compare results with experimental data for different slit gap widths.

The data shown here have been measured in April 2011 by scanning a two-dimensional waveguide through the focus. The beamline was operated in low beta mode at a photon energy of $13.8 \mathrm{keV}$. We will use a rather naïve and an improved slit model:

Naïve slit model First we consider the case of "perfect slits", that just cut the beam without any diffraction. In the simulation the mirror has been shortened appropriately. For a gap width of $50 \mu \mathrm{m}$, this corresponds to an effective mirror length of $12.5 \mathrm{~mm}$. The slit model is illustrated in figure 3.10 (a), while 3.10 (b) shows the simulated curve (red line) and the experimental data (blue, dashed line). Clearly, the main features of the central spot are in good agreement; but the experimental data show two additional peaks at approximately $\pm 2 \mu \mathrm{m}$. Similar scans for different gap widths show that these peaks have a nearly constant intensity and vanish at large gap widths.

Improved slit model The experimental set-up consists of "scatterless slits" [67] that are specifically designed to reduce stray radiation using tilted Germanium brackets. In an improved slit mode, we will study triangular brackets as shown in figure 3.11 (a). The incoming beam is not fully absorbed by the slit; in fact, at a photon energy of $13.8 \mathrm{keV}$ the first $30 \mu \mathrm{m}$ of the slit bracket show an integrated transmission of roughly $10^{-3}$. Due to oblique incidence,

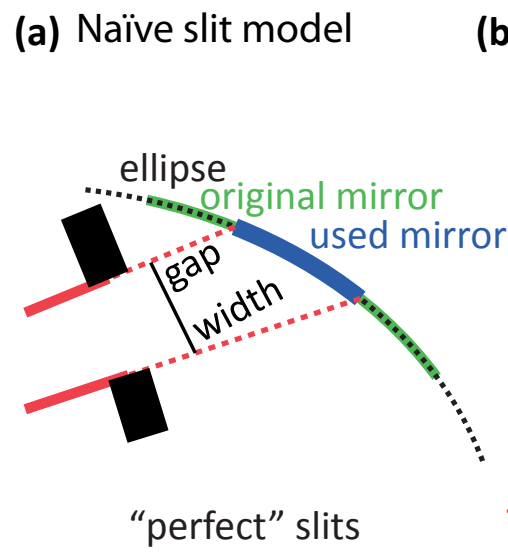

(b) Measured / simulated focus

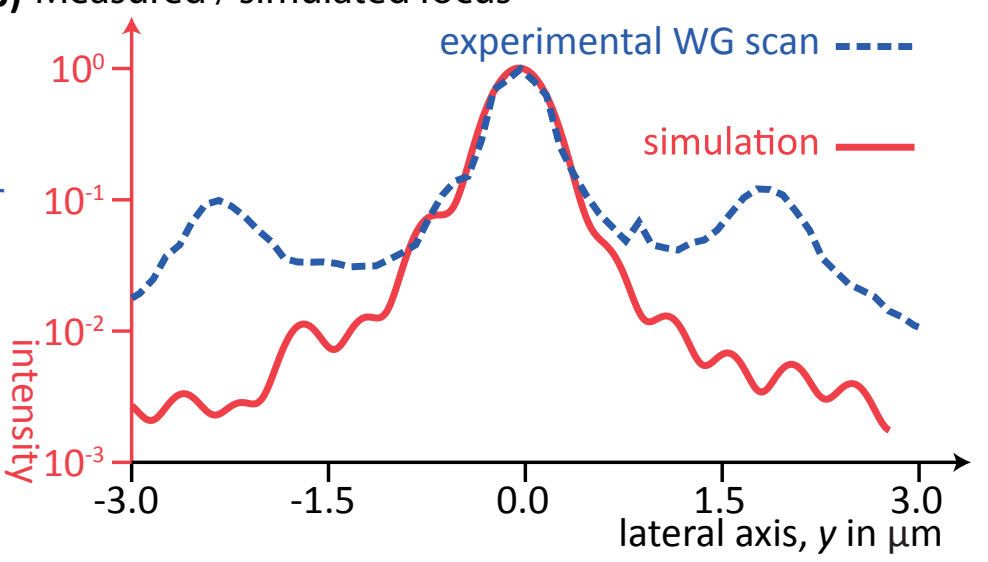

Figure 3.10: Naiive slit model, illustrated in (a), and the simulated intensity in the focal plane ( $b$, red), together with experimental data (blue, dashed). 
refraction occurs. From the peak position at $\approx \pm 2 \mu \mathrm{m}$ and the focal distance of $200 \mathrm{~mm}$, a refraction angle $\phi \approx 10 \mu \mathrm{rad}$ can be estimated. The index of refraction for Germanium at this photon energy is $n \approx 1-5.06 \times 10^{-6}$. Following figure 3.11 (a),

$$
\alpha=-\phi / \delta
$$

Putting in the numbers, the tapering angle can be estimated to be $\alpha \approx 65^{\circ}$.

The simulation has been enhanced using this slit model in the following way:

- The incoming illumination is projected on the slit,

- and multiplied by a transmission function depending on the local thickness.

- This modulated illumination on the mirror surface is conventionally propagated to the focal plane.

Using the scheme from chapter 2, a partially coherent propagation including this improved slit model has been carried out. Figure 3.11 (b) compares the same experimental WG scan from figure 3.10 with a simulation of the more realistic slit mode; the horizontal gap width is $50 \mu \mathrm{m}$. Again the prediction based on simulation is shown with red lines, the experimental WG scan with blue dashed lines. The simulated degree of coherence with respect to the optical axis is shown as a green dotted line.

A large defocus-region of the set-up in figure 3.11 is shown in figure 3.12, with the optical axis $x$ ranging from $-3 \mathrm{~mm}$ to $3 \mathrm{~mm}$ around the focal plane. The small aperture enlarges

\section{(a) Improved slit model}

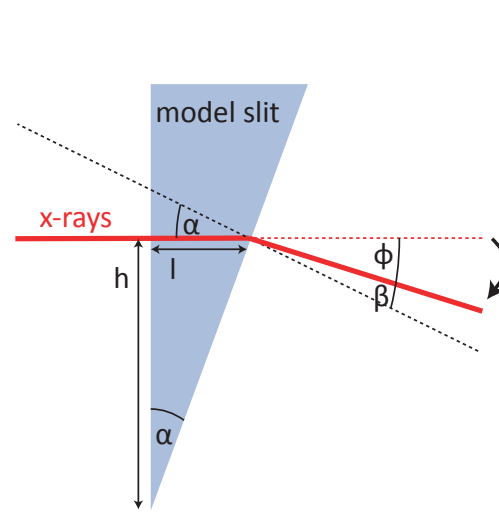

(b) Measured / simulated focus

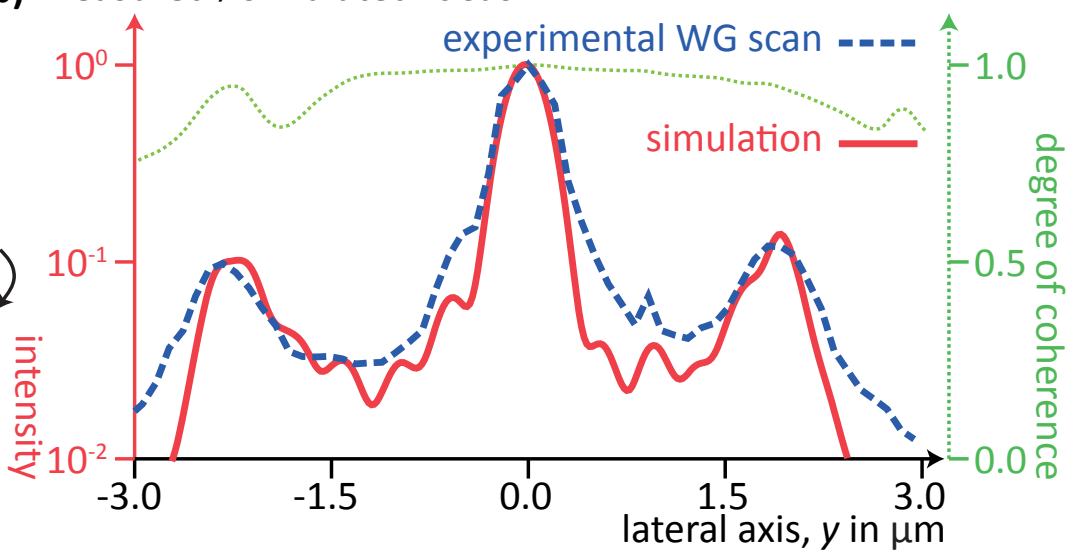

Figure 3.11: The same as in figure 3.10, but for the improved slit model of scatterless slits. Simulated and experimental data are in good agreement. A quantitative statement is given in the text and in table 3.4. The green dotted line shows the computed degree of coherence with respect to the optical axis. 
(a) skbhg $=50 \mu \mathrm{m}$

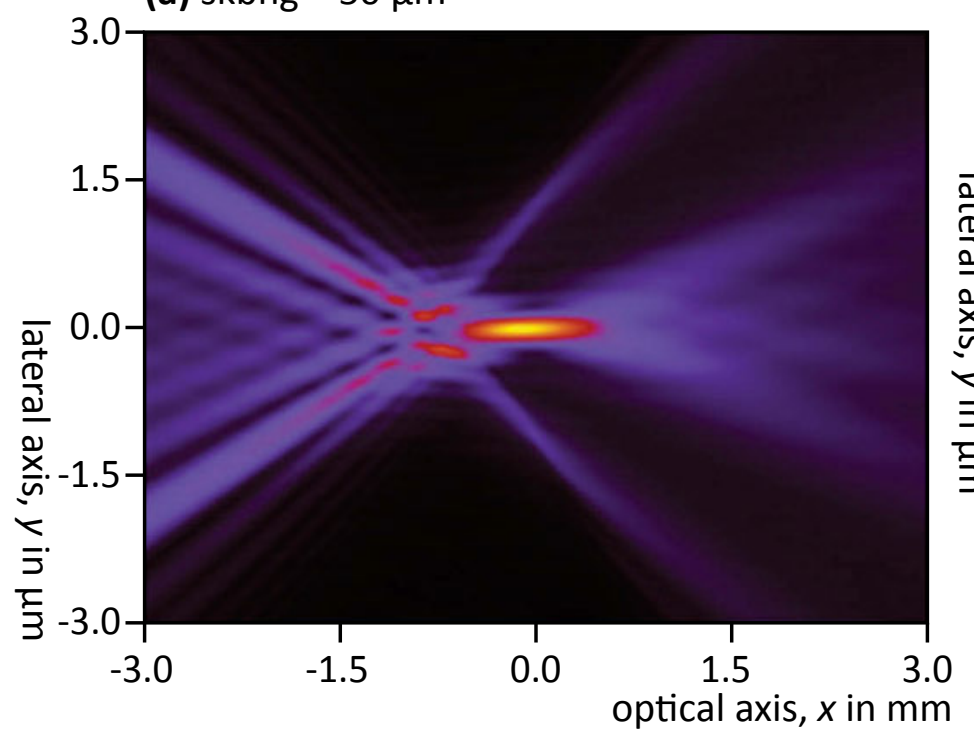

(b) skbhg $=8 \mu \mathrm{m}$

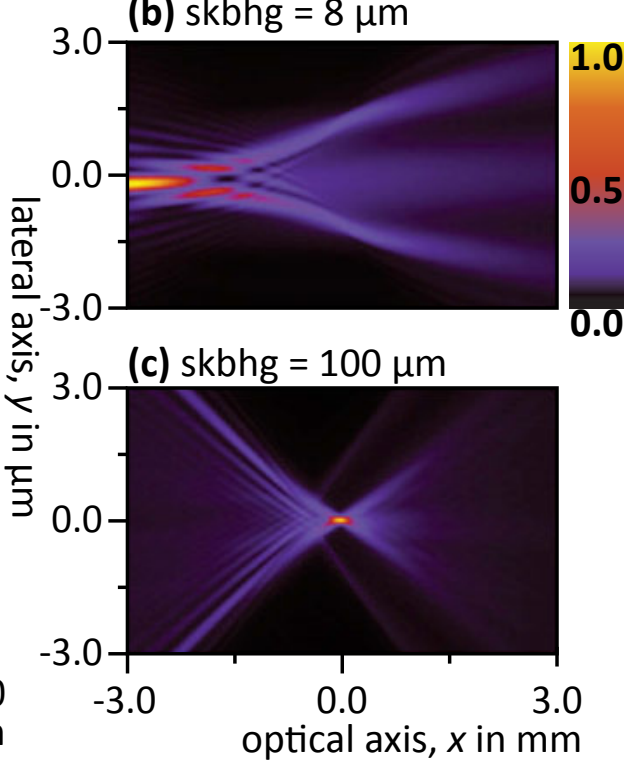

Figure 3.12: Two-dimensional intensity distribution of the set-up shown in figure 3.11, in a large defocus region of $x \in\{ \pm 3 \mathrm{~mm}\}$. The two side lobes in the focal plane can be distinguished as individual "rays". Slit gaps are (a) $50 \mu \mathrm{m}$, (b) $8 \mu \mathrm{m}$, and (c) $100 \mu \mathrm{m}$. Illumination of a low beta source has been assumed.

the depth of focus to one millimetre. The additional peaks are clearly visible as two rays that interfere with the central beam just in front of the focus.

For a small gap of only $8 \mu \mathrm{m}$, figure 3.12 (b), the central beam is very weak and a focus about $3 \mathrm{~mm}$ in front of the nominal focus plane appears due to interference of the refracted beams. At $100 \mu \mathrm{m}$, the relative intensity in the side lobes is weak.

Using this improved slit model, simulation and experimental data are now in good agreement along the full width. A quantitative comparison is shown in table 3.4 based on the Kullback-Leibler divergence $(K L d)$, known from information theory. For two discrete probability distributions $p(x)$ and $q(x)$, the $\operatorname{KLd}(p, q)$ is defined as [68]

$$
\operatorname{KLd}(p, q):=\sum_{x} p(x) \log \frac{p(x)}{q(x)}
$$

Intensity distributions can be interpreted as the probability distribution of finding a photon at a specific location. Hence after normalisation ", we can use the KLd to compare simulation and experiment. Note that the KLd is not symmetric in $p(x)$ and $q(x)$. We will highlight the meaning of this value using basic concepts from the field of information theory, cf. [68]. The $\mathrm{KLd}$ is used as an alternative to the $\chi^{2}$ test; this is advantageous because the KLd is related

$$
\text { I } \sum p(x)=1
$$


to the maximum likelihood estimator for Poisson processes. However, the experimentally measured diode current is not fully Poisson distributed. Essential definitions are given now.

Entropy in information theory Given a finite alphabet $\Sigma$ of symbols ("pixels"), a random variable $X$ on the alphabet and a probability mass function ("normalised intensity") $p(x)=$ $\operatorname{Pr}\{X=x\}, x \in \Sigma$. Then the entropy $H(X)$ of this random process is defined as

$$
H(X)=-\sum_{x} p(x) \log p(x)
$$

Optimal code Given now a "sentence on this alphabet" (corresponding to a "scan of intensity in the focal plane"), we seek an optimal (i.e. shortest) description. In information theory it is shown that there exists an optimal code with length $L$ such that

$$
H(X) \leq L<H(X)+1
$$

so the entropy of the stochastic process is a lower bound on the description length. How such codes can be constructed has been shown by Huffman [69].

Kullback-Leibler divergence in information theory The KLd is also known as the relative entropy between two probability distributions $p(x)$ and $q(x)$. Given one distribution, $\mathrm{KLd}$ is a measure of how good we can predict the other distribution. "It is the reduction in the uncertainty of one random variable due to the knowledge of the other" [68]. Assume a random process $X$ over an alphabet $\Sigma$ and a Huffman code based on $q(x)$. If the actual source is described by the distribution $p(x) \neq q(x)$, the code is not optimal. The KullbackLeibler divergence $\operatorname{KLd}(p, q)$ is a measure of the non-optimality, i.e. quantifies how many additional bits are needed to describe $p(x)$ with the wrong model based on $q(x)$.

Kullback-Leibler divergence in the focal plane Suppose we want to detect individual photons with a one-dimensional pixel detector in the focal plane and store the position (i.e. pixel number) of each "click", using an optimal code. Based on the simulated intensity distribution, we have a model $q(x)$ of the photons' distribution on the pixels and can construct a Huffman code. But in reality, the photons will be distributed by (the unknown, but experimentally observed) $p(x)^{\|}$. So the KLd values given in table 3.4 quantify how many additional bits of storage are needed to record the photons' position, distributed according to $p(x)$, if we assume they are distributed according to the model $q(x)$. The smaller the

\footnotetext{
" "Experimentally observed" means that we treat the data as the truth, while "unknown" reminds us of uncertainties like noise.
} 


\begin{tabular}{r|llll|lll} 
Gap width & Defocus & Backg. & G. Amp. & G. $\sigma$ & $H$ (exp.) & KLd(naïve) & KLd(imp.) \\
\hline $8 \mu \mathrm{m}$ & $0.2 \mathrm{~mm}$ & 0.0007 & 0.008 & $3.1 \mu \mathrm{m}$ & 3.98 & 0.60 & 0.0356 \\
$50 \mu \mathrm{m}$ & $1.2 \mathrm{~mm}$ & 0.0020 & 0.014 & $2.5 \mu \mathrm{m}$ & 3.48 & 0.22 & 0.0279 \\
$100 \mu \mathrm{m}$ & $1.1 \mathrm{~mm}$ & 0.0005 & 0.021 & $3.1 \mu \mathrm{m}$ & 3.38 & 0.41 & 0.0409
\end{tabular}

Table 3.4: Kullback-Leibler divergence and fit parameters for the two slit models at different gap widths; see figures 3.10 and 3.11 for the $50 \mu \mathrm{m}$-entry. Parameters are explained in the text.

$\mathrm{KLd}$, the better our model intensity distribution describes reality. These values have to be compared with $p(x)$ 's entropy that gives the average description length in Bits.

Using the improved slit model, a KLd of 0.0279 is achieved for the gap width of $50 \mu \mathrm{m}$, while the naïve model would "waste about 0.2 bits per photon". Table 3.4 shows KLd values for similar measurements at gap widths of $8 \mu \mathrm{m}(0.04$ vs. 0.60$)$ and $100 \mu \mathrm{m}(0.04$ vs. 0.41). In addition to the improved slit model, a constant background (given in the column "Backg.") and a Gaussian of height "G. Amp" and width "G. $\sigma$ " has been added to the simulated intensities to further reduce the $\mathrm{KLd}$, and hence describe experimental data better. Best accordance has not been found in the simulated focal plane, but in a small defocus of $0.1 \ldots 1.2 \mathrm{~mm}$, as stated in the table.

This might be explained considering additional uncertainties in the experimental set-up: the slit gap could be slightly off-centre, so the mirror might be illuminated asymmetrically. Also the focal plane is not known with highest accuracy; alignment of the whole KB set-up is uncertain in several degrees of freedom.

Table 3.4 also gives the entropy $H$ of the experimental data, according to (3.7), which has been evaluated after normalisation of the 101 data points $\left(\sum I=1\right)$. The two side lobes receive nearly the same intensity for all gap widths, since they are illuminated by finite transmission in the slit brackets. The central peak is illuminated by the gap - and thus scales with the gap width. So at large gaps, the intensity distribution is peaked in the centre, the side lobes' relative contribution becomes negligible. But this simply means that one can more easily "predict where the photon hits the focal plane" - the entropy drops, as calculated from the data and shown in the column " $H$ (exp.)".

Degree of coherence In figure 3.11 (b), the degree of coherence with respect to the focal point is shown by the green, dotted line. Indeed, closing the slits improves coherence properties. Figure 3.13 shows experimental far-fields of a multilayer Laue lens, illuminated with two different slit gaps. At a large gap width of $380 \mu \mathrm{m}$ in (a, top), the pattern is washed out; a more coherent illumination, gap width of $80 \mu \mathrm{m}$ in (a, bottom), shows significantly higher visibility of very small periodicity in reciprocal space. In part (b), intensity has been integrated vertically and plotted horizontally. With reduced aperture, a fine structure 
becomes apparent. Its periodicity of roughly seven pixels suggests a diffracting structure of about $380 \mathrm{~nm}$, which corresponds to the whole MLL size. A visibility of about 0.5 can be expected.

The MLL has been designed by Aike Ruhlandt for his Bachelor's thesis [70] and has been built by Tobias Liese [44]. Further analysis is beyond this thesis [45].

Focus simulations for point-sources as well as extended sources have been analysed, including real structure effects. The mirror simulations, combined with the scheme from chapter 2, allow for quantitative predictions of the partially coherent intensity distribution in the focal region. Using the improved slit model, we can better understand and exploit experimental data. Simulation and experiment have been compared quantitatively.
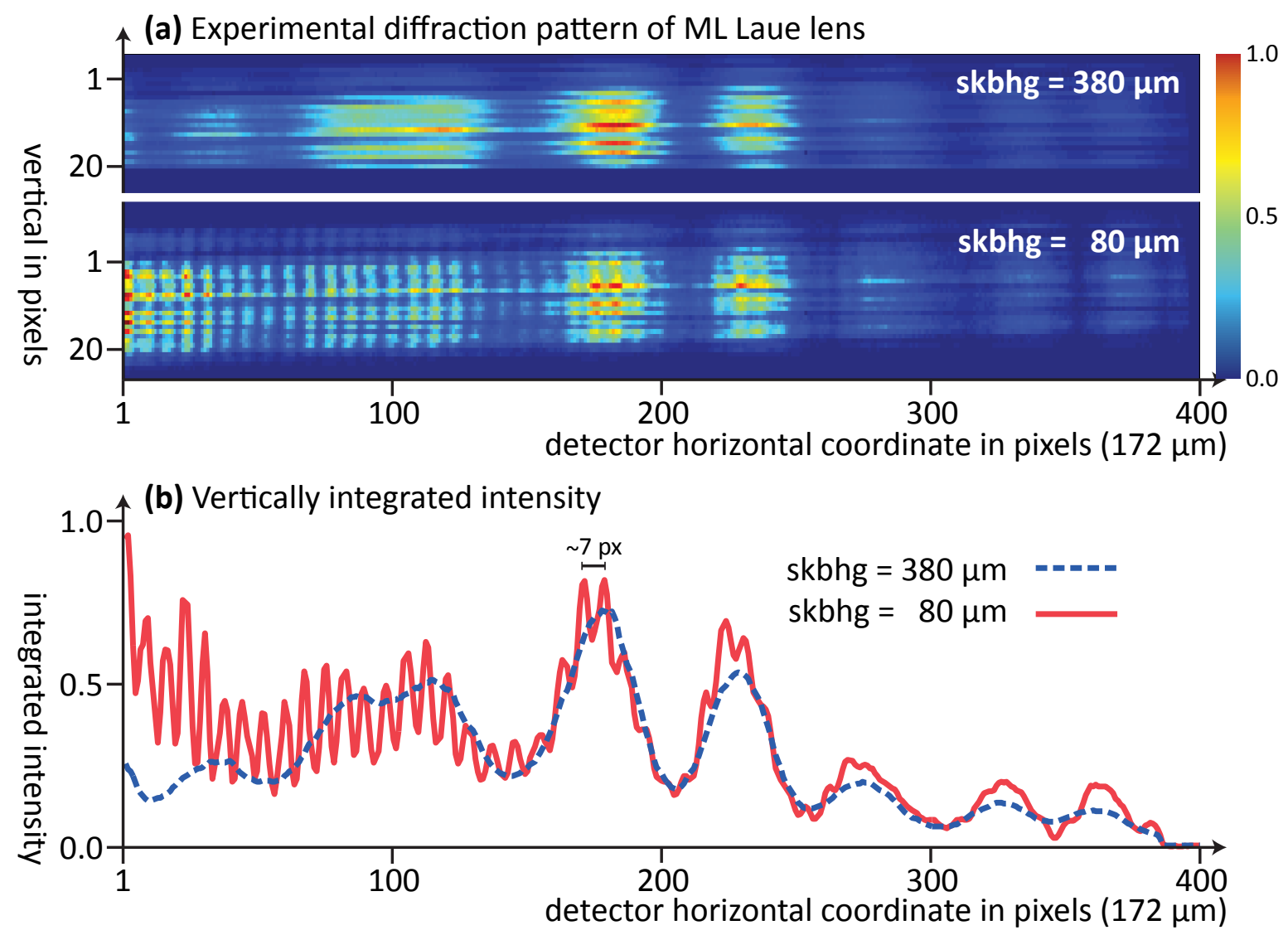

Figure 3.13: Experimental far-fields of a multilayer Laue lens in the KB focus, illuminated with a KB gap width of $380 \mu \mathrm{m}(a$, top) and $80 \mu \mathrm{m}$ (a, bottom). In (b), intensity has been integrated vertically and plotted horizontally for the two slit gap widths. Increasing the degree of coherence, smaller interference structures become visible. 


\subsection{Defocus properties}

The described KB mirror system at the P10 coherence beamline at PETRA III is part of a dedicated set-up for propagation and waveguide based imaging. In such experiments, the specimen is placed in the divergent beam in defocus, usually several millimetres behind the focal plane; thus it is illuminated by a diverging beam. The detector in the far-field then measures a highly magnified "image"; but the measured intensity pattern encodes both amplitude (absorption contrast) and phase (phase contrast) of the sample. Only with numerical means is it possible to reconstruct interesting information.

A rich literature about reconstruction algorithms, their applications, and limitations has been published in the recent past $-[30,66,71-74]$, to list a few.

In the following sections we present experimental and numerical results regarding the $\mathrm{KB}$ system's defocus properties. Some open questions under active research are summarised at the end of this section.

\subsubsection{Crossed KB geometry}

So far we have treated only one mirror of the Kirkpatrick-Baez focusing set-up. Now we generalise to the three-dimensional case, combining independently focused fields in horizontal and vertical direction. Whilst in the experimental set-up the incoming beam hits both mirrors sequentially, the "KB assumption" is that the two dimensions decouple. The simulation is carried out twice, for each mirror separately; the two line-cuts of intensity (e.g. in the focal plane or some defocus) then are crossed and multiplied. Mathematically, this is a tensor product of two vectors, each holding the complex valued amplitude of the independent line cuts.

An example is shown in figure 3.14. In part (a), the two-dimensional intensity distribution is shown as a linear grey-scaled image. In the simulation, both height deviation profiles and partial coherence effects have been included. An experimental near-field measurement

\begin{aligned} Quantity & Value & Quantity & Value \\ \hline photon energy & $7.9 \mathrm{keV} &$ operational mode & low beta \\ defocus (simulation) & $32 \mathrm{~mm} &$ defocus (experiment) & $500 \mathrm{~mm} \\$ field of view (sim) & $60 \times 40 \mu \mathrm{m} &$ field of view (exp) & $1.0 \times 0.8 \mathrm{~mm}\end{aligned}$

Table 3.5: Parameters for the simulation and experiment shown in figure 3.14. Simulated defocus distance is smaller to overcome numerical problems. 

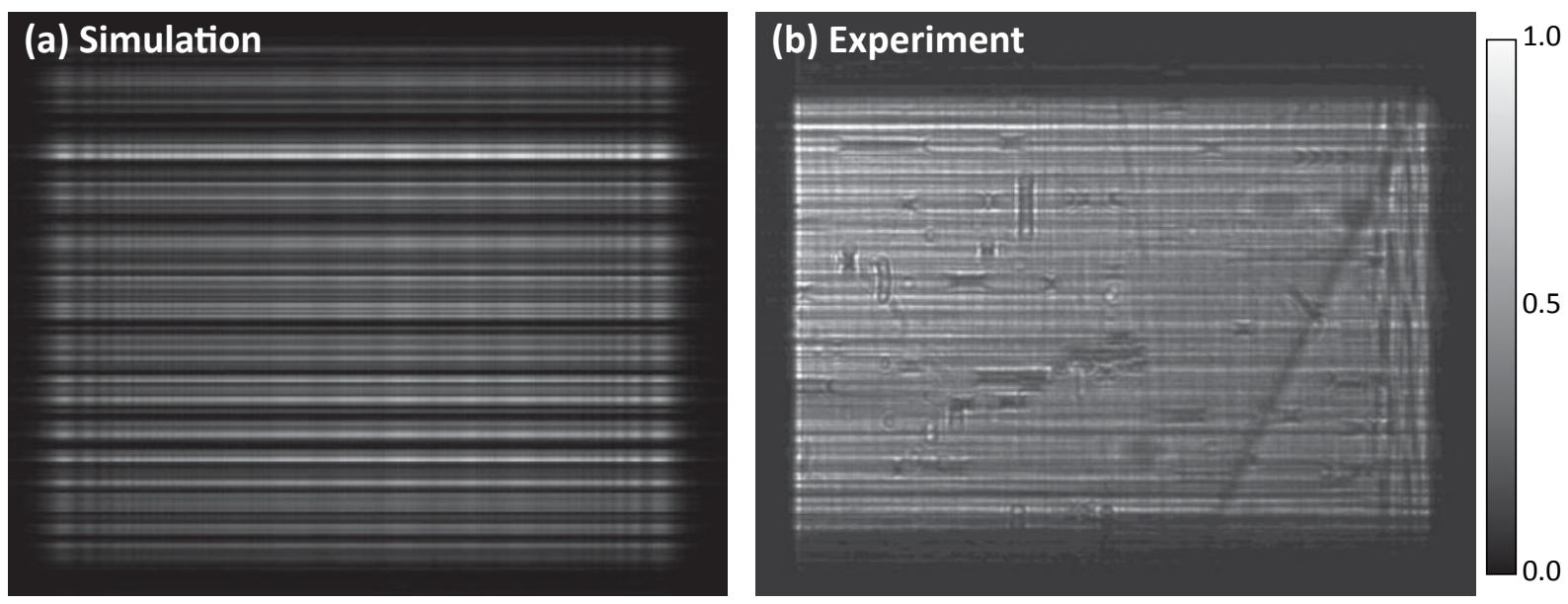

Figure 3.14: Simulated (left) and experimental (right) near-field of the P10 KB mirror set-up; intensity shown on a linear grey-scale.

is shown in figure 3.14 (b). The parameters are shown in table 3.5. Note that defocus distances differ to overcome numerical noise in the simulation. The intensity distribution is comparable, nonetheless.

\subsubsection{Figure errors and Fresnel fringes}

The numerical simulation allows for a distinction of artefacts due to figure errors and propagation. The horizontal lines are present also for different defocus distances and can be attributed to the figure errors. A direct comparison to derivatives of the height deviation profile has not been carried out. Vertical stripes at the left and right border are mainly Fresnel fringes due to propagation, that get weaker for larger propagation distances; some of the faint vertical stripes in the centre can be attributed to HFM's figure errors.

Isolated two-dimensional features in the experiment can be attributed to dust and other small impurities. This effect could be modelled if both mirrors are treated as two-dimensional surfaces, which is not possible with the used simulation code.

\subsubsection{Gouy's phase shift}

Using the stochastic approach of partial coherence presented in chapter 2, not only the averaged intensity and the degree of coherence, but also the phases of all realisations in an ensemble can be analysed. In this subsection we present findings concerning the "stochastic Gouy phase", viz. the phase of the focused wave field along the optical axis. The Gouy 
phase has already been introduced in subsection 3.2.4, then for a coherent point-source.

Due to stochastic illumination, the "phase" in each point in space is random; therefore we use the average relative phase $\varphi(x)$ of the stochastic ensemble, defined in section 2.3:

$$
\varphi(x)=\left\langle\phi_{r}(x)\right\rangle=\left\langle\arg \left(\sum w_{n} c_{n}^{\text {rand }} u_{n}(x)\right)-\phi(F)\right\rangle .
$$

where $\phi(F)$ means the instantaneous phase in the focus point. It can be shown that the phase in the focus of a point-source has a value of $-\pi / 2$ [17], relative to a focusing lens.

After phase unwrapping (see section 2.3), the individual relative phases of an ensemble consisting of $10^{4}$ realisations have been sorted into histograms; these are shown as a greyshade on a logarithmic scale in figure $3.15(\mathrm{a}-\mathrm{d})$. The histograms have bin sizes of $4 \mu \mathrm{m}$ along the optical axis and 0.1 rad in the relative phase. The first three simulations are based on the P10-HFM geometry for source-sizes of $\sigma_{\mathrm{h}}=\{1,12,36\} \mu \mathrm{m}$, while $(\mathrm{d})$ is calculated for the P10-VFM mirror at the nominal source size of $\sigma_{v}=6 \mu \mathrm{m}$. The coloured lines are representative "trajectories" of selected relative phase realisations of the ensemble. The calculations have been carried out using ideal mirror profiles.

For the small, nearly fully coherent source, only small fluctuations of the stochastic Gouy phase along the optical axis are present; that means that the phase relations between different points on the optical axis are nearly deterministic and follow the Gouy phase of a point-source. The intermediate source size of $\sigma_{\mathrm{h}}=12 \mu \mathrm{m}$ shows some fluctuations on the order of up to $2 \pi$; a minor fraction of the realisations show purely random phase relations that reduce visibility in an interference experiment. At the nominal horizontal source size, the relative phase has a large variance; some realisations show a phase randomness of more than $3 \pi$ in the considered region. In the vertical geometry, the nominal source size gives rather clean phase distributions with low randomness.

To quantify the randomness of the relative phases $\phi_{r}(x)$, its standard deviation $\Delta \varphi(x)$, defined as

$$
\Delta \varphi(x)=\sqrt{\left\langle\phi_{r}(x)^{2}\right\rangle-\left\langle\phi_{r}(x)\right\rangle^{2}}
$$

can be used. This gives the variance of the random phase along the optical axis. The evaluation of $\Delta \varphi(x)$ for the two geometries and the different source sizes is shown in figure 3.15 (e). Near the focus, the randomness of the phase vanishes by design.

The Gouy phase phenomenon has been generalised to the case of spatially partial coherence. Random phase relation fluctuations have been quantified as the standard deviation of the relative phase along the optical axis and studied for two focusing mirror set-ups. 

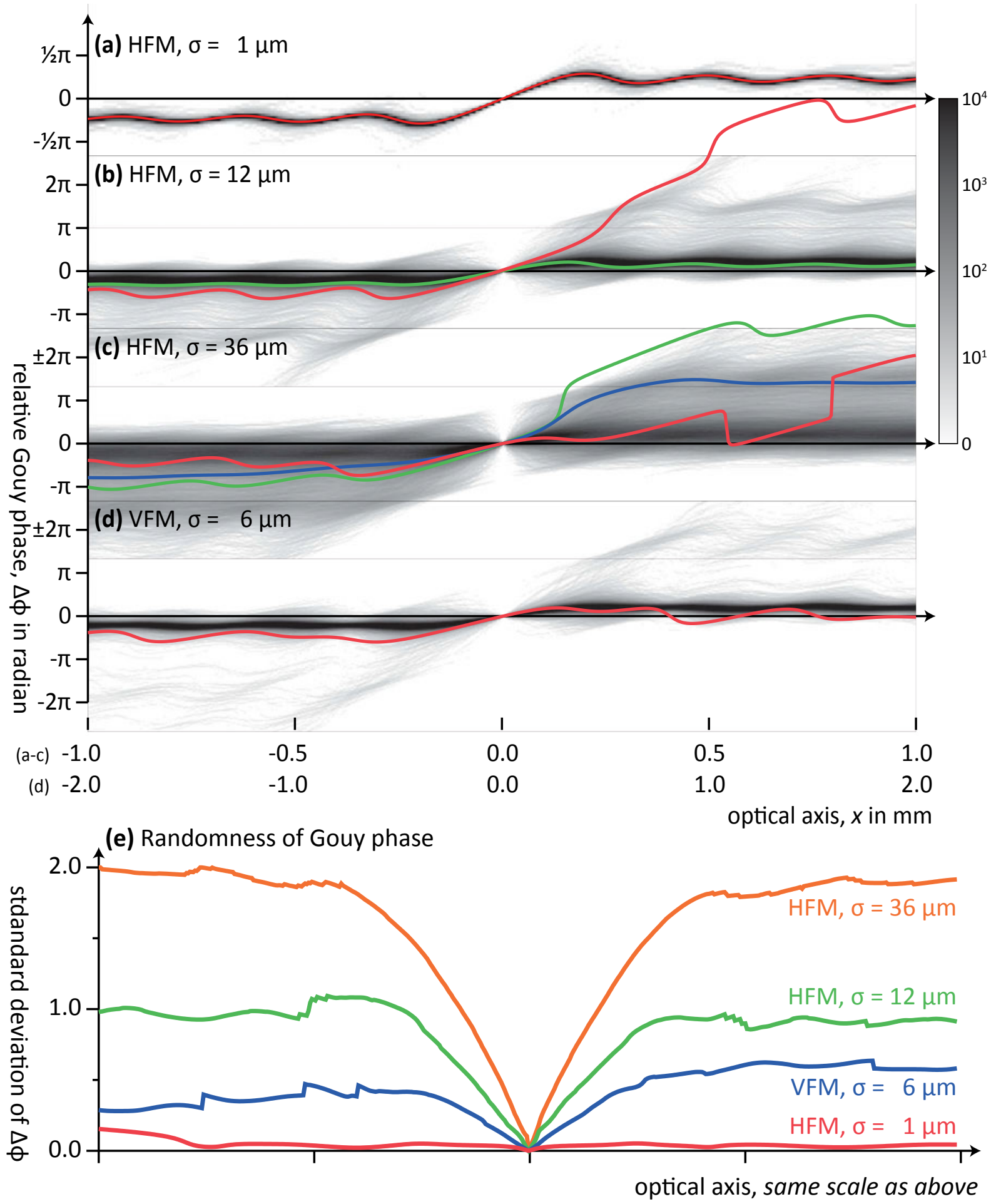

Figure 3.15: Gouy phase predicted at partial coherent illumination. In (a-d), histograms of relative phase along the optical axis are shown for different illumination parameters, on a logarithmic greyscale, and some exemplary trajectories; (e) shows the standard deviation of Gouy phase along the optical axis, for the configurations (a-d). Colours in (a-d) are only to distinguish exemplary trajectories. 


\section{Open questions}

Here we summarise some topics regarding propagation based imaging using focused synchrotron radiation that are open questions of research.

At present, the perhaps most important questions deal with the influence of figure errors and partial coherence. Experimental intensity data have to be preprocessed due to imperfections of the detector and set-up. But it is not clear how to correct for figure errors of the KB mirror system in propagation based imaging.

Currently, the standard-procedure used at the P10 beamline includes a division of the measured intensity image (with sample) by an empty (image without sample). But the Fresnel number of propagating defects depend on the defects' size (spatial frequency); experimental experience strongly suggests that a simple empty correction is not applicable, cf. [75]. For large magnifications and hence small $z_{1}$ (see figure 2.4), Fresnel fringes dominate and might shift significantly due to small vibrations in the set-up. In addition, the illumination function may not be sufficiently constant (an important assumption of ptychographic methods) due to fluctuations of the particle orbit in the synchrotron and vibrations upstream (optics like monochromators etc.).

Furthermore, phase retrieval methods rely on a deterministic phase of a coherent source. Just recently, a "polyCDI" method has been used to reconstruct a test image [76], illuminated with a broadband spectrum. This allowed for significant reduction in exposure time. The incorporation of spatial coherence properties is under discussion. 


\section{Chapter 4}

\section{Multilayer Mirrors}

\subsection{Introduction}

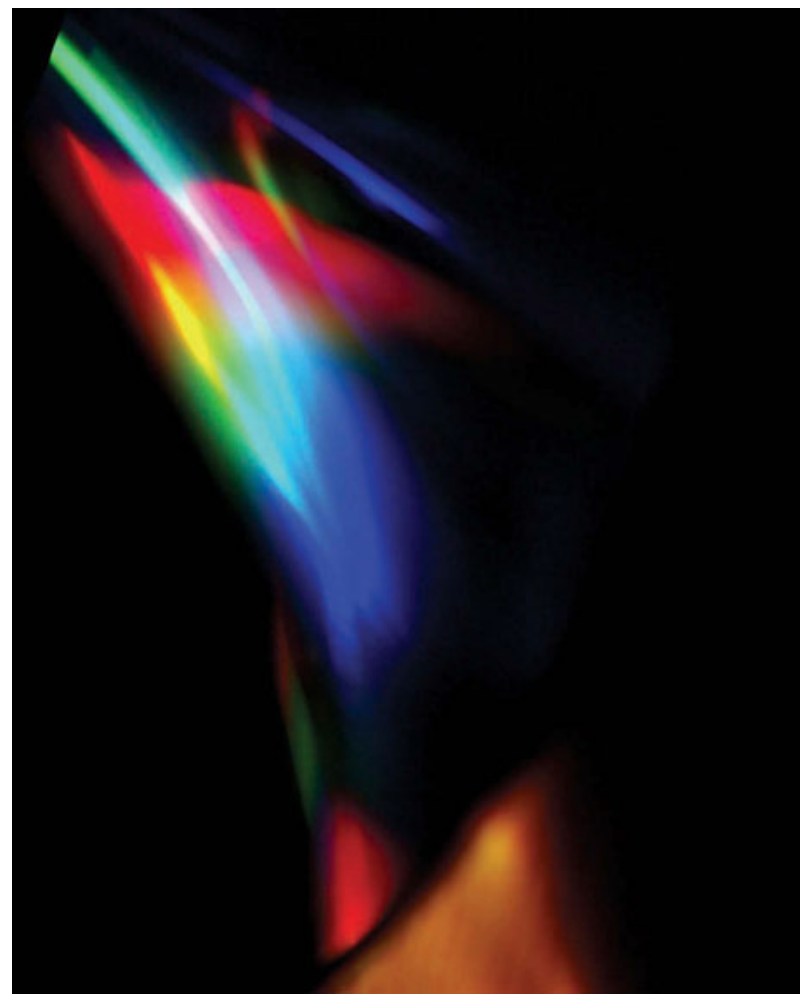

Total reflection mirrors (TRMs) show high reflectivity for small gracing angles of incidence $\theta<\theta_{c}$ only; in a focusing set-up (see chapter 3 ), the local angle of incidence $\theta(s)$ changes with surface coordinate $s$. The geometrical numerical aperture (NA) can be approximated by the projected length $\sin \theta \cdot L$ of the mirror, for some average $\theta$. But the reflectivity decreases rapidly beyond the critical angle; therefore the effective NA is limited. The vanishing reflectivity of a TRM is illustrated in figure 4.1 for an experimental set-up.

This limit may be overcome by using a multilayer mirror (ML mirror), consisting of up to hundreds of small bilayers of two materials with spacings of order few nanometres. If the layer spacing $\Lambda$ matches the Bragg condition, $\Lambda(s)=\lambda / 2 \sin \theta(s)$, greatly enhanced reflectivity as known from crystals can be expected. However, the local layer spacing has to match the local angle of incidence. Furthermore, due to refraction, the Bragg condition has to be modified. The principle of ML mirrors is illustrated in figure 4.2. One distinguishes the kinematical or Born approximation (a) of single reflectivity at each layer from ray-tracing techniques (b), where multiple reflections up to an upper bound are considered. In this work, an approach based on dynamical theory (c) is used, where two wave fields are coupled by volume diffraction of the ML medium.

Multilayer deposition at the ESRF As we will see, many bilayer spacings on the order of $2 \mathrm{~nm}$ have to be deposited on substrates several centimetres in length. For optimal performance of the reflective focusing optics, the layers have to be uniform, with allowed 


\section{(a) KB mirror far-field}

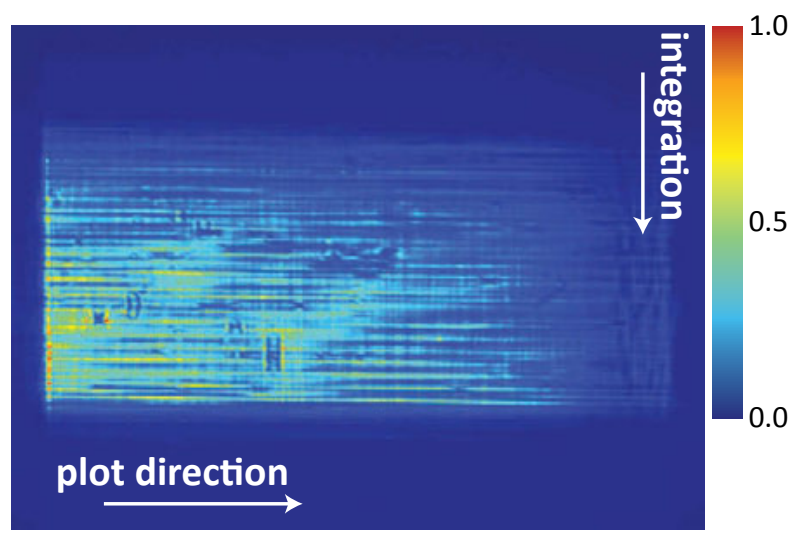

(b) Fresnel reflectivity

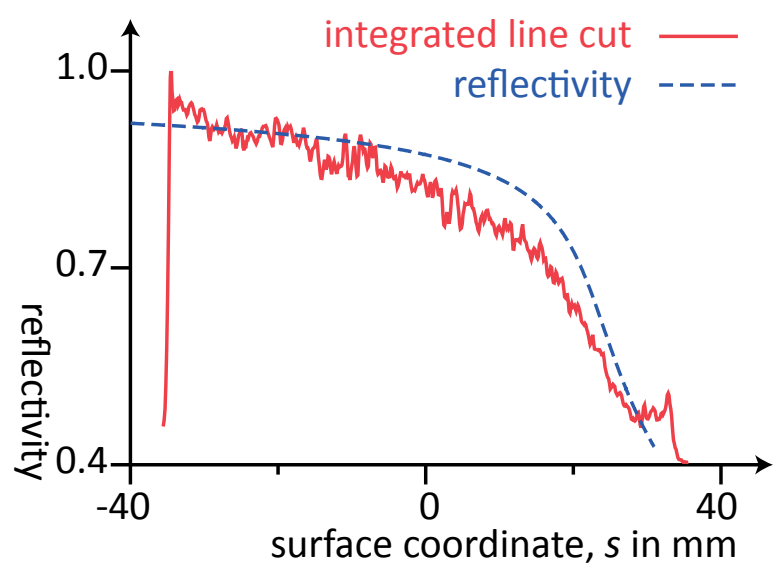

Figure 4.1: Limited reflectivity of a total reflection mirror, operated above critical energy. (a) Experimental far-field distribution, measured at a photon energy of $15 \mathrm{keV}$; (b) vertically integrated horizontal line-cut of the reflectivity (red line) and calculated Fresnel reflectivity along the mirror surface (blue, dashed line). The set-up is the horizontal focusing mirror at the P10 beamline, PETRA III.

(a) Kinematical theory

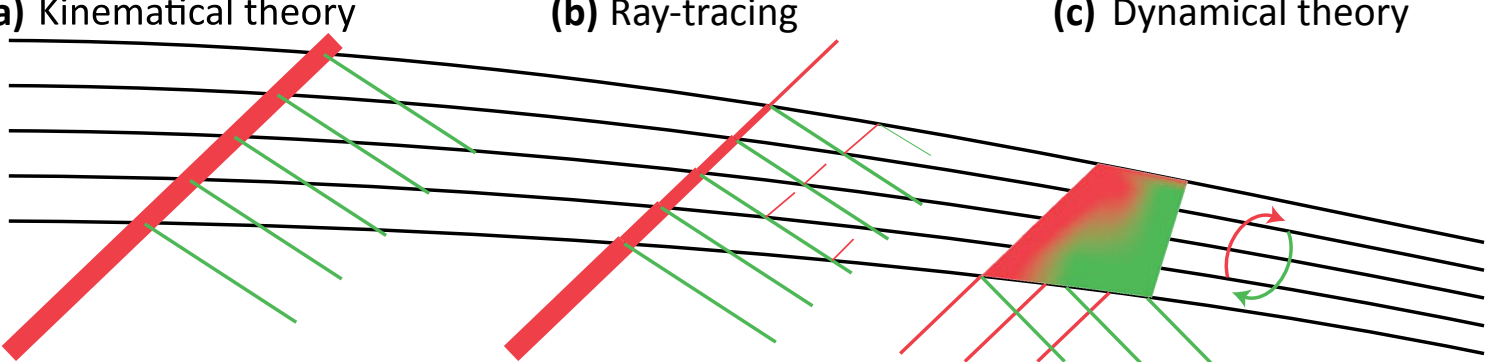

Figure 4.2: Functional principle of reflective multilayers: (a) In kinematical or Born approximation, each of the confocal layers contribute to the overall reflectivity, since the angle of incidence is large compared to the critical angle. Multiple reflections are neglected. (b) Using ray-tracing techniques, multiple reflections are taken into account. (c) Dynamical theory respects multiple diffraction of wave fields. 


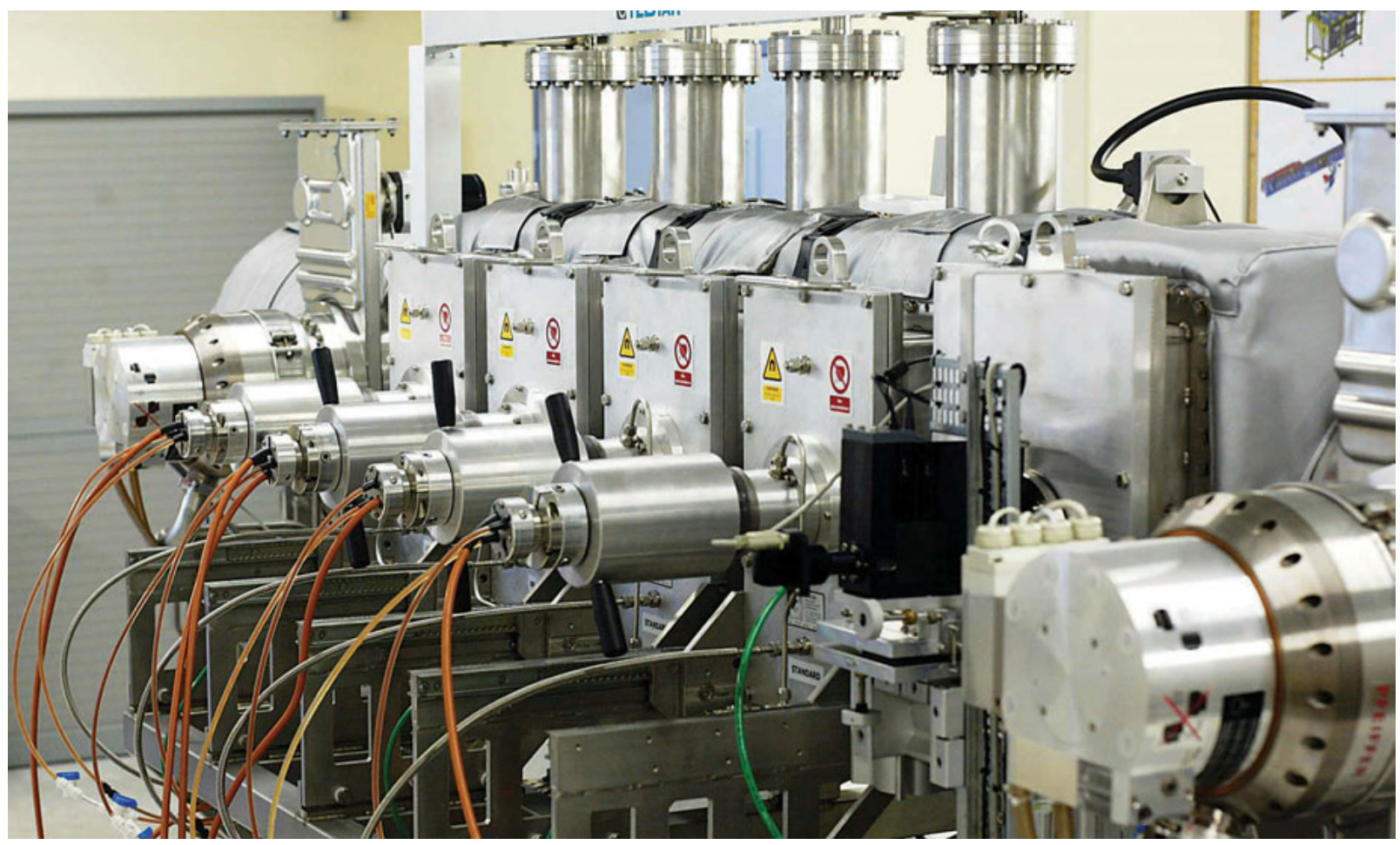

Figure 4.3: Photograph of the magnetron sputtering machine in the multilayer laboratory of the ESRF. Visible are the four cathodes and the long vacuum chamber.

tolerances well below an ångström $(0.1 \mathrm{~nm})$. The most common deposition technique for high aspect ML mirrors is magnetron sputtering. This method combines a stable operation with high deposition rates. The sputtered particles possess high kinetic energies which helps to achieve both smooth and dense thin layers [47]. The multilayer laboratory at the ESRF was upgraded in recent years. The new magnetron sputtering set-up allows for uniform depositions of thin films with layer thickness in the nanometre range. Using masks and programmable velocity variations of the sliding carriage carrying the mirror substrate, gradients in lateral direction and depth are possible $[77,78]$. The deposition machine holds four targets that can be used for sputtering. Most multilayers are built using alternating layers of tungsten $(\mathrm{W})$ and boron carbide $\left(\mathrm{B}_{4} \mathrm{C}\right)$, with typical single-layer thicknesses around $2 \mathrm{~nm}$, and 25 to 100 bilayers in total. Substrates of sizes up to $1 \mathrm{~m} \times 0.15 \mathrm{~m}$ can be treated. An image of the machine can be found in figure 4.3 .

In this chapter we will first derive a theoretical description based on two-beam approximation analogue to the Takagi-Taupin (TT) theory [79-82] well known for crystals; this is carried out in section 4.2. An analogue set of coupled differential equations has been used for nearly half a century in x-ray topography and related fields. Using elliptical coordinates, a convenient description will be derived that is of similar form as the theory known for flat crystals. The refraction correction $[46,47,83]$ described by a modified Bragg condi- 
tion will be incorporated within this wave-optical approach in subsection 4.3.2, after some generalisations of the TT equations have been introduced.

Our method of solving the TT system is described and discussed in section 4.4. A tabular description of the approach, and especially the algorithm, can be found in appendices $A$ and D. A comparison of our approach with analytical results from the flat case are summarised in section 4.5. Results for curved multilayer mirrors then follow in section 4.6.

\subsection{Takagi-Taupin equations in curved ML geometry}

As a theoretical model to describe focusing $M L$ mirrors a framework well known from dynamical theory of x-ray diffraction in crystals has been chosen. It is based on the TakagiTaupin theory and the two-beam approximation. The incoming wave envelope is denoted by $\psi_{0}$, while the reflected wave envelope is called $\psi_{1}$. The incoming wave $\psi_{0}$ is determined on the mirror's surface by the illumination function. The reflected wave is propagated from the surface to the focus.

These $\psi_{0}$ and $\psi_{1}$ are complex valued envelope functions, to be multiplied by cylindrical waves diverging from the source or converging to the focus. They interact with the layer lattice, described by (pseudo) Fourier coefficients $\chi_{ \pm 1}$.

As a first step we will consider confocal elliptical layers and then include deviations from this default profile. Such deviations are necessary to account for refraction, and are described by a modified Bragg condition $[46,47,83]$. Now we look at different descriptions of $x$-ray diffraction in crystals and multilayers.

Diffraction limit The diffraction limited spot size $\Delta$ in linear optics is given as

$$
\Delta=0.88 \cdot \lambda / N A
$$

where $\mathrm{NA}=n \sin \varepsilon$ is the numerical aperture, $n$ the index of refraction and $\varepsilon$ the angle of collimation. The factor 0.88 gives $\Delta$ as a full width at half maximum (FWHM) for a onedimensional focus, in thin-lens approximation a sinc ${ }^{2}$-function. The Rayleigh criterion, the distance of first minimum from the central spot, gives a prefactor of 1.00 in one dimension; a circular aperture results in an Airy disk, with a prefactor of 1.22 (Rayleigh criterion). For curved $\mathrm{ML}$ mirrors, the angle of collimation $\varepsilon$ can be approximated as the difference of the local angles of incidence at the left and right edge, $\varepsilon=\theta_{2}-\theta_{1}{ }^{*}$. Bragg's law gives the layer

*This neglects curvature, however. 
spacing $\Lambda$ as a function of (vacuum) wavelength $\lambda$ and angle of incidence $\theta$,

$$
\Lambda=\lambda / 2 \sin \theta
$$

the diffraction limit can be estimated by the layer spacing gradient [46]:

$$
\Delta=\frac{0.88}{\Lambda_{2}^{-1}-\Lambda_{1}^{-1}}
$$

Hence, the lower limit of the spot size is related to the smallest structure in the ML. A similar criterion is known from ML Laue lenses [8].

Kinematic theory, Born approximation As a first approximation, one can treat all surfaces independently and add up reflectivity coefficients. Since reflected waves interfere with each other, one would add amplitudes. Neglecting multiple diffraction is known as the (first) Born approximation and the basis for kinematical diffraction developed about one hundred years ago.

Ray-tracing techniques follow geometrical beams through the ML structure, accounting for refraction and reflection at each interface. More sophisticated methods known as phaseray-tracing include phase jumps and optical path lengths. Consider the family of all ray paths of discrete length $2 n-2, n \geq 1$, that enter the ML structure and are transmitted or reflected $2 n-1$ times, and leave the ML at the surface after these $2 n-1$ steps. The number of rays in this family is then given as the $n$th Catalan number $c_{n}$ [84-86]. A recursive algorithm to generate such families of rays is given in appendix $B$.

Dynamical theory of x-ray diffraction Allowing the reflected wave to re-reflect, in the end allowing multiple reflection and thus coupling of wave fields results in the dynamical theory of x-ray diffraction. Basically we treat a set of excited wave fields and their interaction with a (discrete) diffracting structure. Modelling this structure as a continuous diffractive volume, the wave fields are coupled to each other at every point in space. Intensity is exchanged between all excited fields. The dynamical theory of x-ray diffraction was mainly shaped by works of Charles Galton Darwin [87], Max von Laue, Paul Peter Ewald and others [88]; historical notes and further references can be found in $[89,90]$. Early reviews are listed in [91]. For an exhaustive discussion of several approximations, read [92].

Two-beam approximation Instead of "many" excited fields, only two are considered, namely the incoming wave and the reflected wave (or diffracted wave). This approach is justified if only one point is sufficiently close to the Ewald sphere [90]. 
Takagi and Taupin The basic assumptions and simplifications of the TT theory are (i) the two-beam approximation and (ii) the representation of the periodic structure by its first Fourier series expansion coefficient; sharp surfaces properly described by a rectangular wave are treated as a sine wave. The sine wave expansion then couples the two wave fields. As we will see later, in the Takagi-Taupin approach it is easy and natural to include distortions of the lattice.

History of TT-theory The Takagi-Taupin theory was developed by Daniel Taupin and Satio Takagi in the 1960s [79-81]. Since then, numerical implementations [93-96] have been developed to study $\mathrm{x}$-ray diffraction of e.g. dislocations and other real-structure effects in crystals. For a more complete review of the last four decades of dynamical diffraction theory, cf. [90]. Recently, the TT theory was used in [43] to optimise ML Laue lenses.

Methods for solving Analytical solutions only exist for very special and "easy" geometries. In the last decades, most numerical simulations have been calculated with finite difference methods $[93,96]$, i.e. by a simple point-to-point integration of the differential equations on a regular grid. To increase accuracy while decreasing run time, non-regular grids have been proposed and used. Here, we will use a regular grid in elliptical coordinates; in "real-space", this also corresponds to a non-regular grid. Numerical solutions based on the finite element method which is most convenient for non-regular grids have been proposed [97], too.

In the following subsection we will derive the fundamental set of coupled differential equations using Takagi-Taupin formalism, but in elliptical coordinates.

Our nomenclature and the steps in the derivation are based on [43]; the choice of elliptical coordinates has been developed together with Jean-Pierre Guigay in lots of fruitful discussions. Claudio Ferrero is thanked for his advice in numerical treatment. Christian Morawe contributed additional insights and developed the modified Bragg equation.

In subsection 4.2.2, deviations from the confocal geometry will be included in the theory. Such deviations may stem from inaccuracies, but here we consider intended deviations for optimisation.

\subsubsection{Fundamental equations}

In this subsection we will derive the Takagi-Taupin equations in the two-beam case in elliptically curved multilayer structures. As in chapter 3 we consider $1+1$ dimensions for convenience; the elliptical ML is considered to be infinitely extended in the perpendicular 
(a) Confocal ellipses

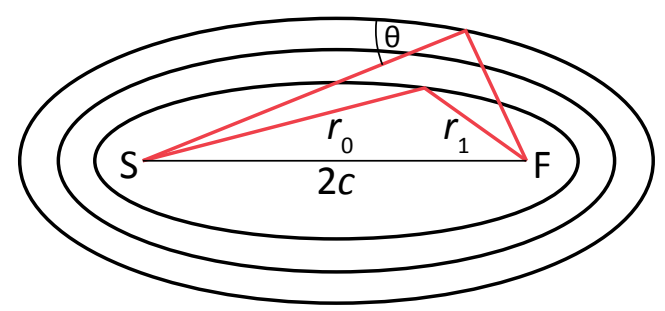

(b) Elliptical coordinates

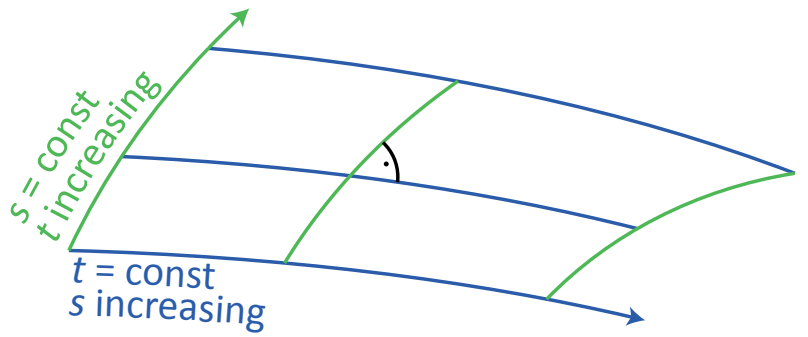

Figure 4.4: Definition of elliptical coordinates (b) describing multilayers designed from nearly confocal ellipses (a) conveniently.

direction. A line-source emits cylindrical waves which look like circular waves emitted by a point-source in the considered plane.

For further simplifications, we only consider scalar wave theory here, so neglect any polarisation effects ${ }^{\dagger}$. As the fundamental equation of scalar diffraction theory, one component $\psi$ of the electromagnetic field obeys the scalar wave equation

$$
\nabla^{2} \psi+k^{2}(1+\chi) \psi=0
$$

Here $k=2 \pi / \lambda$ is the wavenumber and $\lambda$ the wavelength in vacuum and $\chi$ is the susceptibility of the medium, which is related to the index of refraction $n$ and the critical angle of total reflection $\theta_{c}$ via

$$
(1+\chi)=n^{2}=(1-\delta+i \beta)^{2} \approx 1-2 \delta=1-\theta_{c}^{2} .
$$

In the calculations, numerical values for $n(\lambda)$ are taken from databases [52].

In a first approximation, the surfaces of focusing multilayer mirrors represent confocal ellipses, see figure 4.4 (a) for an illustration. Thus we will introduce a system of adequate coordinates: ellipses with $s=$ const. and hyperbolas with $t=$ const., as shown in figure 4.4 (b). These two kinds of curves form an orthogonal basis in two dimensions.

With the distances $r_{0}$ from the source to a point inside the ML structure, and $r_{1}$ from this point to the focus, we define $(s, t)$ via

$$
r_{0}+r_{1}=2 t, \quad r_{0}-r_{1}=2 s
$$

by virtue of this definition, we get

$$
\begin{aligned}
& r_{0}=t+s, \\
& r_{1}=t-s ;
\end{aligned} \quad \nabla f(s, t)=\left(\begin{array}{l}
\alpha \partial_{s} \\
\beta \partial_{t}
\end{array}\right) f(s, t) ; \quad \begin{aligned}
& \alpha(s, t)=\cos \theta(s, t), \\
& \beta(s, t)=\sin \theta(s, t) .
\end{aligned}
$$

\footnotetext{
$\dagger$ In fact, polarisation dependent scattering often is included by an appropriate factor, often denoted as $C$, in literature.

${ }^{\ddagger} \mathrm{cf}$. [98], equations $(21.1 .1,21.4 .1,21.4 .2)$.
} 
The function $f(s, t)$ is arbitrary, but sufficiently smooth. The coefficients $\alpha$ and $\beta$ are the inverse scale factors of this choice of coordinates and are represented by

$$
\alpha^{2}=\frac{c^{2}-s^{2}}{t^{2}-s^{2}}, \quad \beta^{2}=\frac{t^{2}-c^{2}}{t^{2}-s^{2}},
$$

with $c$ being half of the (direct) distance between source and focus. The Laplacian operator $\nabla^{2}$ can be expressed as

$$
\begin{aligned}
\nabla^{2} f & =\alpha \beta\left[\partial_{s}\left(\frac{\alpha}{\beta}\left(\partial_{s} f\right)\right)+\partial_{t}\left(\frac{\beta}{\alpha}\left(\partial_{t} f\right)\right)\right] \\
& =\alpha^{2}\left(\partial_{s}^{2} f\right)+\beta^{2}\left(\partial_{t}^{2} f\right)+\frac{\alpha^{2} s}{s^{2}-c^{2}}\left(\partial_{s} f\right)+\frac{\beta^{2} t}{t^{2}-c^{2}}\left(\partial_{t} f\right)
\end{aligned}
$$

Within the two-beam approximation, the scalar wave field $\psi$ is considered to be the superposition of two components. These components are modelled as cylindrical waves $P_{0}=e^{i k r_{0}}$ (diverging from the source) and $P_{1}=e^{-i k r_{1}}$ (converging to the focus) with slowly varying envelopes $\psi_{0}$ and $\psi_{1}$ :

$$
\begin{aligned}
\psi & =\sum_{h^{\prime}} \psi_{h^{\prime}} P_{h^{\prime}} \approx \psi_{0} P_{0}+\psi_{1} P_{1}=\psi_{0} P_{0}+\psi_{1} P_{0} \Phi_{\overline{1}} \\
& =P_{0}\left(\psi_{0}+\psi_{1} \Phi_{\overline{1}}\right) .
\end{aligned}
$$

The gradient of the phase in $\Phi_{\overline{1}}$ can be interpreted as a local lattice vector that diffracts the incoming cylindrical wave into the outgoing, reflected, wave. $\Phi_{1}$ on the other hand re-diffracts energy back from $\psi_{1}$ to $\psi_{0}$; the two beams thus are coupled. The strength of the coupling depends on the achieved resonance.

For convenience we have defined phase factors $\Phi_{h}$, where the index $\overline{1}$ means -1 :

$$
\Phi_{0}=1, \quad \Phi_{\overline{1}}=e^{-2 i k t}, \quad \Phi_{1}=e^{2 i k t}, \quad \Phi_{\overline{2}}=e^{-4 i k t} .
$$

With this ansatz we favour solutions to the wave equation that comply with our a priori knowledge - there is exactly one point-source - and the requirement that there shall be one focus. All possible solutions that do not fit into this model are not of interest and thus discarded by design.

Up until now we have considered two waves propagating in vacuum. The ML structure is now described by its susceptibility function $\chi(s, t)$. Since the layers are built in a quasi-periodic manner, we will describe $\chi$ by its Fourier series expansion. In the two-beam approximation, only terms of zeroth and first order are considered, hence

$$
\chi=\chi_{0}+\sum_{h \neq 0} \chi_{h} e^{i \tilde{h} t} \approx \chi_{0}+\chi_{\overline{1}} e^{-i \tilde{h} t}+\chi_{1} e^{i \tilde{h} t} .
$$


For a definition of the Fourier components $\chi_{0}, \chi_{\overline{1}}$, and $\chi_{1}$, see appendix I. Here, $\tilde{h}$ is related to the reciprocal lattice vector in the elliptical coordinates and given by the transformed layer thickness $\tilde{\Lambda}$. The transformation of coordinates gives an additional factor of $\sin \theta(s, t)$ that depends on position:

$$
\begin{aligned}
\tilde{h} & =\frac{2 \pi}{\tilde{\Lambda}}, \\
\tilde{\Lambda} & =\tilde{\Lambda}^{\mathrm{B}} \approx \Lambda^{\mathrm{B}} \times \sin \theta=\frac{\lambda}{2 \sin \theta} \times \sin \theta=\frac{\lambda}{2}, \\
\Rightarrow \tilde{h} & =\frac{4 \pi}{\lambda}=2 k,
\end{aligned}
$$

where we have used Bragg's law; optimised ML structures are built by use of a modified Bragg equation $[46,47,83]$ resulting in a layer thickness $\Lambda^{\mathrm{mB}}$, which in the framework of this theory is regarded as a distortion of the Bragg lattice. We will introduce the modified Bragg equation further below as an additional phase factor in a pseudo-Fourier series. Neglecting refraction and phase shifts, the Bragg law is fulfilled everywhere, since the confocal ellipses follow the geometrical path condition.

Using Bragg's law we have derived the Fourier expansion phase factor exp(i2kt), which is constant for constant $t$. But these are just the ellipses in figure 4.4. Hence the "Bragg layers" of a focusing ML mirror are confocal ellipses.

With the phase terms $\Phi_{h}$ defined above we can write the Fourier expansion of the susceptibility $\chi$ as $\chi=\chi_{0} \Phi_{0}+\chi_{\overline{1}} \Phi_{\overline{1}}+\chi_{1} \Phi_{1}$. The product $\chi \psi$ appears in the wave-equation; this would consist of $2 \times 3=6$ terms. But we neglect those proportional to either $\Phi_{\overline{2}}$ or $\Phi_{1}$; these phase factors are not associated with the two beams $P_{0}$ and $P_{1}=P_{0} \Phi_{\overline{1}}$. Using this approximation, we write

$$
\begin{aligned}
& P_{0}\left(\psi_{0} \Phi_{0}+\psi_{1} \Phi_{\overline{1}}\right) \cdot\left(\chi_{0} \Phi_{0}+\chi_{\overline{1}} \Phi_{\overline{1}}+\chi_{1} \Phi_{1}\right) \\
= & P_{0} \Phi_{0}\left(\chi_{0} \psi_{0}+\chi_{1} \psi_{1}\right)+P_{0} \Phi_{\overline{1}}\left(\chi_{0} \psi_{1}+\chi_{\overline{1}} \psi_{0}\right)+P_{0} \Phi_{\overline{2}} \chi_{\overline{1}} \psi_{1}+P_{0} \Phi_{1} \chi_{1} \psi_{0} \\
\approx & P_{0}\left(\chi_{0} \psi_{0}+\chi_{1} \psi_{1}\right)+P_{0} \Phi_{\overline{1}}\left(\chi_{0} \psi_{1}+\chi_{\overline{1}} \psi_{0}\right) .
\end{aligned}
$$

The index of $\Phi$ is the difference of the indexes of $\chi$ and $\psi$. Within this two-beam approximation, the scalar wave equation (4.3) reads

$$
\begin{aligned}
0= & \left(\nabla^{2}+k^{2}\right) \psi+k^{2} \chi \psi \\
= & \left(\nabla^{2}+k^{2}\right)\left(\psi_{0} P_{0}+\psi_{1} P_{1}\right) \\
& +k^{2} P_{0}\left(\chi_{0} \psi_{0}+\chi_{1} \psi_{1}\right)+k^{2} P_{1}\left(\chi_{0} \psi_{1}+\chi_{\overline{1}} \psi_{0}\right) .
\end{aligned}
$$

This sum of the form $f_{0} P_{0}+f_{1} P_{1}$ with oscillating $P^{\prime}$ 's vanishes if both $f_{0}=0$ and $f_{1}=0$, so we split the sum into two equations and obtain

$$
\left.\begin{array}{l}
\left(\nabla^{2}+k^{2}\right)\left(\psi_{0} P_{0}\right)+k^{2} \chi_{0} \psi_{0} P_{0}+k^{2} \chi_{1} \psi_{1} P_{0} \\
\left(\nabla^{2}+k^{2}\right)\left(\psi_{1} P_{1}\right)+k^{2} \chi_{0} \psi_{1} P_{1}+k^{2} \chi_{1} \psi_{0} P_{1}
\end{array}\right\}=0
$$


With the transformation law for the Laplacian, we get

$$
\nabla^{2}\left(\psi_{0} P_{0}\right)=\alpha^{2}\left(\partial_{s}^{2}\left(\psi_{0} P_{0}\right)\right)+\beta^{2}\left(\partial_{t}^{2}\left(\psi_{0} P_{0}\right)\right)+\frac{\alpha^{2} s}{s^{2}-c^{2}}\left(\partial_{s}\left(\psi_{0} P_{0}\right)\right)+\frac{\beta^{2} t}{t^{2}-c^{2}}\left(\partial_{t}\left(\psi_{0} P_{0}\right)\right)
$$

For clarity, we will only derive the terms $\propto \alpha^{2} P_{0}$ here:

$$
\begin{aligned}
& \alpha^{2}\left[\left(\partial_{s}^{2} \psi_{0}\right) P_{0}+2\left(\partial_{s} \psi_{0}\right)\left(\partial_{s} P_{0}\right)+\psi_{0}\left(\partial_{s}^{2} P_{0}\right)+\frac{s}{s^{2}-c^{2}}\left(\left(\partial_{s} \psi_{0}\right) P_{0}+\psi_{0}\left(\partial_{s} P_{0}\right)\right)\right] \\
\approx & \alpha^{2} P_{0}\left[2\left(\partial_{s} \psi_{0}\right) i k-\psi_{0} k^{2}+\frac{s}{s^{2}-c^{2}}\left(\left(\partial_{s} \psi_{0}\right)+\psi_{0} i k\right)\right] \\
\approx & \alpha^{2} P_{0}\left[2 i k\left(\partial_{s} \psi_{0}\right)-k^{2} \psi_{0}+\frac{s}{s^{2}-c^{2}} i k \psi_{0}\right]
\end{aligned}
$$

the analogue expression $\propto \beta^{2} P_{0}$ is

$$
\beta^{2} P_{0}\left[2 i k\left(\partial_{t} \psi_{0}\right)-k^{2} \psi_{0}+\frac{t}{t^{2}-c^{2}} i k \psi_{0}\right]
$$

The term $\partial_{s}^{2} \psi_{0}$ has been neglected since we postulate slowly varying envelopes; furthermore a term $\partial_{s} \psi_{0}$ is considered small since all other terms have a factor of $k$ or $k^{2}$, which is of order $10^{10}$. Re-combining both expressions, the Laplacian of $P_{0}$ can be written as

$$
\begin{aligned}
\nabla^{2}\left(\psi_{0} P_{0}\right) & =\alpha^{2} P_{0}\left[2 i k\left(\partial_{s} \psi_{0}\right)-k^{2} \psi_{0}+\frac{s}{s^{2}-c^{2}} i k \psi_{0}\right] \\
& +\beta^{2} P_{0}\left[2 i k\left(\partial_{t} \psi_{0}\right)-k^{2} \psi_{0}+\frac{t}{t^{2}-c^{2}} i k \psi_{0}\right] .
\end{aligned}
$$

The Laplacian for the second wave field, $P_{1}$, is

$$
\begin{aligned}
\nabla^{2}\left(\psi_{1} P_{1}\right) & =\alpha^{2} P_{1}\left[2 i k\left(\partial_{s} \psi_{1}\right)-k^{2} \psi_{1}+\frac{s}{s^{2}-c^{2}} i k \psi_{1}\right] \\
& +\beta^{2} P_{1}\left[-2 i k\left(\partial_{t} \psi_{1}\right)-k^{2} \psi_{1}-\frac{t}{t^{2}-c^{2}} i k \psi_{1}\right] .
\end{aligned}
$$

A parallel derivation of $\nabla^{2}\left(\psi_{h} P_{h}\right)$ can be found in appendix A, both for confocal ellipses and for those with arbitrary distortions.

It can be shown that

$$
\frac{\alpha^{2} s}{s^{2}-c^{2}} \pm \frac{\beta^{2} t}{t^{2}-c^{2}}=\frac{1}{t \pm s}=: \pm 2 \gamma^{ \pm}
$$

Qua definitione, the complete Laplacian of the incoming wave field's envelope, $P_{0}$, can be approximated by

$$
\nabla^{2}\left(\psi_{0} P_{0}\right) \approx 2 i k P_{0}\left[\alpha^{2} \partial_{s} \psi_{0}+\beta^{2} \partial_{t} \psi_{0}+\frac{i k}{2} \psi_{0}+\gamma^{+} \psi_{0}\right] .
$$


Operating with $\left(\nabla^{2}+k^{2}\right)$ on both $\psi_{0} P_{0}$ and $\psi_{1} P_{1}$, we get

$$
\begin{aligned}
& \left(\nabla^{2}+k^{2}\right)\left(\psi_{0} P_{0}\right) \approx 2 i k P_{0}\left[\alpha^{2} \partial_{s} \psi_{0}+\beta^{2} \partial_{t} \psi_{0}+\gamma^{+} \psi_{0}\right] \\
& \left(\nabla^{2}+k^{2}\right)\left(\psi_{1} P_{1}\right) \approx 2 i k P_{1}\left[\alpha^{2} \partial_{s} \psi_{1}-\beta^{2} \partial_{t} \psi_{1}-\gamma^{-} \psi_{1}\right] .
\end{aligned}
$$

The scalar wave equation now can be written as

$$
\left.\begin{array}{l}
2 i k P_{0}\left(\alpha^{2} \partial_{s} \psi_{0}+\beta^{2} \partial_{t} \psi_{0}+\gamma^{+} \psi_{0}\right)+k^{2} \chi_{0} \psi_{0} P_{0}+k^{2} \chi_{1} \psi_{1} P_{0} \\
2 i k P_{1}\left(\alpha^{2} \partial_{s} \psi_{1}-\beta^{2} \partial_{t} \psi_{1}-\gamma^{-} \psi_{1}\right)+k^{2} \chi_{0} \psi_{1} P_{1}+k^{2} \chi_{\overline{1}} \psi_{0} P_{1}
\end{array}\right\}=0
$$

Reordering, we get the Takagi-Taupin equations in elliptical geometry for focusing Bragg-multilayer mirrors:

$$
\begin{aligned}
& \left(\alpha^{2} \partial_{s}+\beta^{2} \partial_{t}\right) \psi_{0}=i\left(u_{0} \psi_{0}+u_{1} \psi_{1}\right)-\gamma^{+} \psi_{0} \\
& \left(\alpha^{2} \partial_{s}-\beta^{2} \partial_{t}\right) \psi_{1}=i\left(u_{0} \psi_{1}+u_{\overline{1}} \psi_{0}\right)+\gamma^{-} \psi_{1}
\end{aligned}
$$

with $u_{h}=k \chi_{h} / 2$. These equations have a similar form as in the flat case, except that the coefficients $\alpha$ and $\beta$ depend on coordinates, see (4.4).

The terms $\gamma^{ \pm}$originate from our choice of cylindrical waves: Since energy is conserved, the intensity (energy flux density) of the diverging $\psi_{0} P_{0}$ has to decrease, while the converging $\psi_{1} P_{1}$ gains intensity. From a mathematical point of view, these terms $\gamma^{ \pm}$are a result of our space-dependent coefficients $\alpha, \beta$. So $\partial_{s}$ and $\alpha \beta^{-1}$ in the Laplacian do not commutate and result in an additional term $s\left(s^{2}-c^{2}\right)^{-1} \psi_{0} \partial_{s} P_{0}$, plus a similar term with $t$ instead of $s$ for $\partial_{t}$.

For typical geometries the terms $\gamma^{ \pm}$are small. Let us neglect this commutator and simply use the following gradients and Laplacians:

$$
\begin{aligned}
P_{0} & =e^{i k(t+s)} & P_{1} & =P_{0} e^{-2 i k t} \\
\nabla P_{0} & =\left(\begin{array}{c}
\alpha \partial_{s} \\
\beta \partial_{t}
\end{array}\right) e^{i k(t+s)}=i k\left(\begin{array}{c}
\alpha \\
\beta
\end{array}\right) P_{0} & \nabla P_{1} & =i k\left(\begin{array}{c}
+\alpha \\
-\beta
\end{array}\right) P_{1} \\
\nabla^{2} P_{0} & =-k^{2}\left(\alpha^{2}+\beta^{2}\right) P_{0}=-k^{2} P_{0} ; & \nabla^{2} P_{1} & =-k^{2} P_{1} ;
\end{aligned}
$$

the resulting Takagi-Taupin equations then read

$$
\begin{aligned}
& \left(\alpha^{2} \partial_{s}+\beta^{2} \partial_{t}\right) \psi_{0}=i\left(u_{0} \psi_{0}+u_{1} \psi_{1}\right), \\
& \left(\alpha^{2} \partial_{s}-\beta^{2} \partial_{t}\right) \psi_{1}=i\left(u_{0} \psi_{1}+u_{\overline{1}} \psi_{0}\right) .
\end{aligned}
$$

In the flat $M L$ case, the coefficients would be $\alpha^{2}=\cos \theta$ and $\beta^{2}=\sin \theta$. The squares in (4.4) stem from the transformation of coordinates, that give additional factors of $\cos \theta$ in $s$-direction and $\sin \theta$ in $t$-direction. 


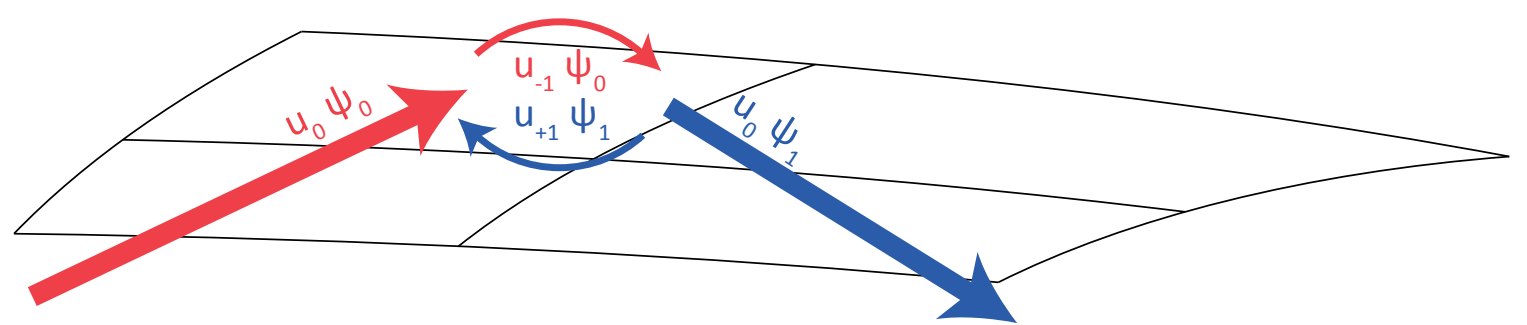

Figure 4.5: Cartoon interpreting the terms in (4.8). The incoming beam $\psi_{0}$, shown in red, propagates through the ML structure; phase shifts and absorption are described by the coefficient $u_{0}$. In a ML of Bragg type, the reflected beam $\psi_{1}$ (blue arrow) is influenced by the same propagation constant. Energy is exchanged back and forth between the two wave fields by virtue of the coefficients $u_{ \pm 1}$, which are Fourier coefficients of the pseudo periodic susceptibility function $\chi(s, t)$.

In a more cartoonish form this system of coupled first order differential equations can be interpreted and illustrated as in figure 4.5.

To summarise our findings:

- The term $i \chi_{0} \psi_{0,1}$ describes propagation of the two fields inside a homogeneous medium; $\chi_{0}$ is the mean susceptibility of the ML structure and results a phase shift and absorption.

- The terms $i \chi_{ \pm 1} \psi_{0,1}$ accounts for coupling: energy is exchanged between $\psi_{0}$ and $\psi_{1}$.

Note that although a ML mirror consists of individual layers with (more or less) sharp boundaries, in the Takagi-Taupin theory the whole volume is capable of diffraction. Hence even a structure composed of an infinitely thin layer will emit a Bragg peak.

The Takagi-Taupin equations in curved elliptically shaped focusing multilayer mirrors have been derived in this subsection; they possess a form well known from the flat crystal case. But due to refraction, perfect ellipses do not yield perfect interference; in the next subsection we will use a generalised Fourier expansion of the susceptibility to describe optimised shapes. 


\subsubsection{Pseudo Bragg conditions}

In this subsection we generalise the Fourier expansion of the susceptibility by introducing a variable phase-factor. In the elliptical coordinate system, confocal elliptical shapes are described by constant values of the coordinate $t$. So in the previous derivation of the TakagiTaupin equations, the susceptibility was assumed to be a periodic function with respect to $t$, with a layer thickness given by Bragg's law. In the Fourier expansion, this was represented by the phase factor $e^{i 2 k t}$, which we will now replace by a more general factor,

$$
\chi=\chi_{0}+\chi_{\overline{1}} e^{-i k(2 t-\varphi)}+\chi_{1} e^{i k(2 t-\varphi)} .
$$

The undisturbed phase term 2ikt corresponds to the simple Bragg law, while ik $\varphi(s, t)$ represents some (smooth) distortion function.

To form Takagi-Taupin equations for quasi-periodic, distorted, curved ML mirrors, the derivatives of this generalised phase exponential are needed. For the sake of simplicity, we will neglect the commutator term in the Laplacian, $\partial_{s}\left(\alpha \beta^{-1}\right) \partial_{s} \Phi$, but introduce the spherical wave term $\gamma^{\prime \pm}$ at the end. More comments will be given in appendix $A$.

Since we need to combine the phase factors of our trial field functions and the susceptibility expansion, we also need to transform

$$
P_{1}^{\prime}=P_{1} e^{i k \varphi(s, t)} .
$$

Physically speaking, the deformed ML structure possesses different local lattice vectors, and hence the diffracted wave changes its direction.

Following appendix $\mathrm{A}$, the simplified gradients and Laplacians of $P_{0}$ and $P_{1}^{\prime}$ read

$$
\begin{gathered}
\nabla P_{0}=i k\left(\begin{array}{c}
\alpha \\
\beta
\end{array}\right) P_{0}, \quad \nabla^{2} P_{0}=-k^{2} P_{0} ; \quad \nabla P_{1}^{\prime}=i k\left(\begin{array}{c}
\alpha\left(1+\varphi_{, s}\right) \\
\beta\left(1-\varphi_{, t}\right)
\end{array}\right) P_{1}^{\prime}, \\
\nabla^{2} P_{1}^{\prime}=-k^{2}\left[\alpha^{2}\left(1+2 \varphi_{, s}+\varphi_{, s}^{2}-i k^{-1} \varphi_{, s s}\right)+\beta^{2}\left(1-2 \varphi_{, t}+\varphi_{, t}^{2}-i k^{-1} \varphi_{, t t}\right)\right] P_{1}^{\prime},
\end{gathered}
$$

where $\varphi_{, s}$ and $\varphi_{, t}$ represent the derivatives of the distortion function $\varphi(s, t)$ with respect to the elliptical coordinates $s$ and $t$.

Putting everything together, the Takagi-Taupin equations of non-elliptical ML mirrors, described by a distortion function $\varphi(s, t)$, can be written as

$$
\begin{aligned}
\left(\alpha^{2} \partial_{s}+\beta^{2} \partial_{t}\right) \psi_{0} & =i\left(u_{0} \psi_{0}+u_{1} \psi_{1}\right)-\gamma^{+} \psi_{0}, \\
\left(\alpha^{\prime 2} \partial_{s}-\beta^{\prime 2} \partial_{t}\right) \psi_{1} & =i\left(\left(u_{0}-\varepsilon^{\prime}\right) \psi_{1}+u_{\overline{1}} \psi_{0}\right)+\gamma^{\prime-} \psi_{1},
\end{aligned}
$$

with

$$
\begin{aligned}
& \alpha^{\prime 2}=\alpha^{2}\left(1+\varphi_{, s}\right), \quad \beta^{\prime 2}=\beta^{2}\left(1-\varphi_{, t}\right), \quad \gamma^{\prime-}=\frac{1}{2}\left(\frac{\beta^{\prime 2} t}{t^{2}-c^{2}}-\frac{\alpha^{\prime 2} s}{s^{2}-c^{2}}\right), \\
& \varepsilon^{\prime}=k \varepsilon / 2, \quad \varepsilon \approx \alpha^{2}\left(2 \varphi_{, s}+\varphi_{, s}^{2}\right)+\beta^{2}\left(-2 \varphi_{, t}+\varphi_{, t}^{2}\right) .
\end{aligned}
$$


The Takagi-Taupin equations have been generalised to non-elliptical shapes by use of a pseudo Fourier expansion. In the next section, we will use this framework and introduce two deviation functions $\varphi(s, t)$ that describe optimised ML structures.

\subsection{Generalised Bragg conditions}

With generalised Takagi-Taupin equations for non-elliptical ML mirrors we will now discuss several model deviation functions $\varphi(s, t)$. We start with a global rotation of the lattice and then use a local rotation, or strain, that is derived from the modified Bragg equation correcting for refraction.

an subsection 4.3.1 we will use the generalised Fourier expansion of the previous subsection to introduce a model deviation function $\varphi(s, t)$ that describes a rotated Bragg lattice. Due to such a rotation, the effective layer spacing is changed: as can be seen in figure 4.6 (a), the geometrical path length of a ray under gracing incidence angle $\theta-\Delta \theta$ is longer than for the original ray of angle $\theta$. Since the optical path length, $l_{\text {opt }}=n l_{\text {geo }}<l_{\text {geo }}$, is not resonant at the nominal Bragg angle, a slight deviation might increase reflectivity.

A modified Bragg equation incorporating a correction term for effects of refraction is being used to build optimised ML mirrors; in subsection 4.3.2 we will show that this correction is equivalent to a local rotation, i.e. a deformation of the $\mathrm{ML}$ structure. The angle of rotation is proportional to the refraction strength $\delta$.

The general procedure is as follows:

1. the ML is designed as before, fulfilling the Bragg condition,

2. but then the lattice is distorted by a deviation function $\varphi(s, t)$;

3. by changing parameters $\{p\}$ in the deviation model $\varphi(s, t,\{p\})$, the $M L$ mirror can be tuned with respect to phase flatness, improved reflectivity, or broad-band reflectivity. 


\subsubsection{Rotated Bragg condition}

By a rotation of the $\mathrm{ML}$ structure, the geometrical path and hence the effective layer spacing can be adjusted to overcome refraction. Figure $4.6(a, b)$ illustrates that a ray penetrating the structure under a grazing incidence angle $\theta-\Delta \theta$ is equivalent to the original ray in a structure with changed layer thickness $\Lambda^{\prime}$. We will now set up a model deviation function $\varphi(s, t, \Delta \theta)$ that describes such (global) rotations.

We work directly in the system of elliptical coordinates $(s, t)$. Then the confocal ellipses - our first guess of an appropriate structure - are described by straight lines with $t=$ const. With the transformation

$$
t \mapsto t+\Delta t(s, t)=t+\left(t-t_{0}\right) \sin \Delta \theta / \sin \theta(s, t),
$$

where $t_{0}$ denotes the entrance surface, a rotation of the lattice around the centre of the surface, by a constant angle $\Delta \theta$, is modelled. The angle $\theta(s)$ is the local angle of incidence, which varies slightly along the entrance surface. See figure 4.6 for the following derivation of this transformation. A more complete discussion of this transformation can be found in appendix D.3.2.

In the initial situation, given by the angle of incidence $\theta$ and the layer spacing $\Lambda^{\mathrm{B}}$, the projected path length inside the $M L$ mirror is given by $I_{1}$ from figure 4.6 (a). A rotation of the $\mathrm{ML}$ can be described by a change of the angle of incidence $\theta \mapsto \theta+\Delta \theta$, with the new path length $I_{2}$. But this new path length can also be achieved for the original angle $\theta$, if the layer spacing $\Lambda^{\mathrm{B}} \mapsto \Lambda^{\mathrm{mB}}$ is changed appropriately, as shown in figure 4.6 (b). Following figure 4.6 (c), the modified layer spacing can be expressed by its old value after scaling of the $t$-coordinate.

So a rotation of the $\mathrm{ML}$ is equivalent to a change of layer spacing; and a change of layer spacing can be described by a transformation of coordinates. We will describe this transformation using a distortion function $\varphi(s, t)$; following subsection 4.2 .2 , this yields a

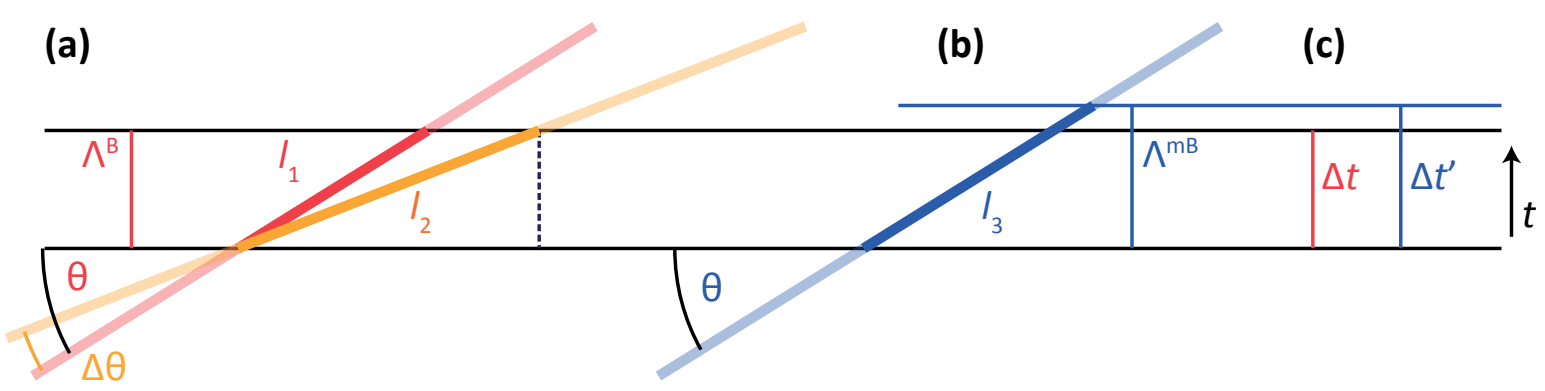

Figure 4.6: Relationship between changed angle of incidence (a), changed layer thickness (b) and a transformation of $t$ coordinate $(c)$. For details see the text. 
pseudo Fourier series expansion of $\chi$ and at last the modified TT equations (4.9).

The transformation of $t$-coordinate is (4.10); for simplicity, let us first neglect $\theta$ 's dependence on coordinates. Then the distortion function $\varphi(s, t)$ is given as

$$
\varphi(t)=2\left(t-t_{0}\right) \Delta \theta / \sin \theta
$$

with the constant angle of rotation $\Delta \theta$ and the "constant" angle of incidence $\theta$. The factor of 2 is introduced because the susceptibility has an inverse thickness of $2 k$. This is related to the $1 / 2$ in Bragg's law.

Within the current approximations, the derivatives of this distortion function are simply

$$
\varphi_{, t}=2 \Delta \theta / \sin \theta, \quad \varphi_{, t t}=\varphi_{, s}=\varphi_{, s s}=0 .
$$

A variant including more terms is given in appendix D.3.2.

The modified Takagi-Taupin equations for a rotated ML mirror read

$$
\begin{aligned}
\left(\alpha^{2} \partial_{s}+\beta^{2} \partial_{t}\right) \psi_{0} & =i\left(u_{0} \psi_{0}+u_{\overline{1}} \psi_{1}\right)-\gamma^{+} \psi_{0}, \\
\left(\alpha^{\prime 2} \partial_{s}-\beta^{\prime 2} \partial_{t}\right) \psi_{1} & =i\left(u_{0}^{\prime} \psi_{1}+u_{1} \psi_{0}\right)+\gamma^{\prime-} \psi_{1}, \\
u_{0}^{\prime} & =u_{0}-\varepsilon^{\prime}, \quad \varepsilon^{\prime}=-4 \Delta \theta \sin \theta k / 2
\end{aligned}
$$

with

$$
\alpha^{\prime 2}=\alpha^{2}, \quad \beta^{\prime 2}=\beta^{2}(1-2 \Delta \theta / \sin \theta),
$$

and the appropriate $\gamma^{\prime}$ as given below (4.9).

Results using this approach and comparisons with analytical solutions based on Parratt's algorithm are presented in section 4.5.

\subsubsection{Modified Bragg condition}

The Bragg equation gives the (bi-)layer spacing $\Lambda^{\mathrm{B}}$ as a function of wavelength $\lambda$ and gracing angle of incidence $\theta$ as

$$
\Lambda^{\mathrm{B}}=\lambda / 2 \sin \theta
$$

In the derivation, the length / in figure 4.7 shall be a multiple of half the wavelength, or $2 I=m \lambda$, for diffraction in the $m$ th order. We will set $m=1$ and only treat the first order of diffraction now. From the definition of the sine, we have $I=\Lambda \sin \theta$ and hence (4.12). 


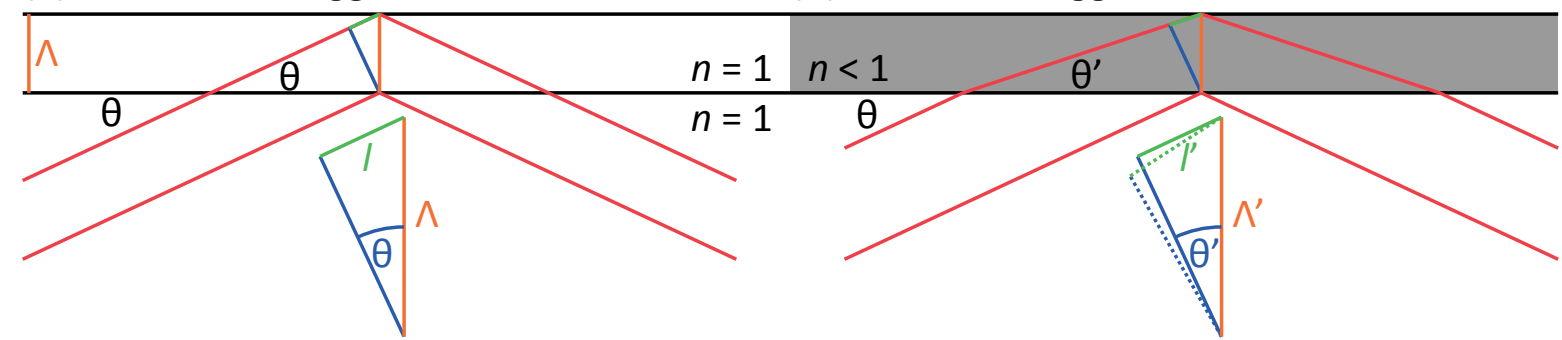

Figure 4.7: The Bragg condition: (a) without refraction, (b) with refraction due to $n<1$ in the medium. Here, the modified Bragg condition is derived for the planar case of two parallel layers; in elliptically curved $M L$, the refraction correction distorts the structure.

The ML mirror consists of multiple bilayers; as an approximation, these can be described by an average index of refraction $n=\Gamma n_{1}+(1-\Gamma) n_{2}$. Here, $\Gamma$ is the ratio of layer thickness of both materials. The incoming beam is then refracted by Snell's law, (3.1), resulting in the distance $l^{\prime}$ and experiences in propagating an optical path length of $n l^{\prime}$ in the (average) medium. Rewriting Snell's law to

$$
\begin{aligned}
& 1=\sin ^{2} \theta^{\prime}+\cos ^{2} \theta^{\prime}=\sin ^{2} \theta^{\prime}+\cos ^{2} \theta / n^{2}, \\
& n^{2} \sin ^{2} \theta^{\prime}=n^{2}-\cos ^{2} \theta,
\end{aligned}
$$

the modified Bragg condition gives the modified layer spacing $\Lambda^{\mathrm{mB}}(s)$, see figure 4.7 (b) [47]:

$$
\wedge^{\mathrm{mB}}(s)=\frac{\lambda}{2 \sqrt{n^{2}-\cos ^{2} \theta(s)}} .
$$

Example Consider a $\mathrm{ML}$ mirror made of tungsten (W) and Boron-carbide $\left(\mathrm{B}_{4} \mathrm{C}\right)$, with equal thickness of both materials. The average index of refraction at an $\mathrm{x}$-ray wavelength of $0.1 \mathrm{~nm}$ is

$$
\begin{aligned}
n_{\mathrm{avg}} & =\left(n_{\mathrm{W}}+n_{\mathrm{B}_{4} \mathrm{C}}\right) / 2 \\
& \approx\left(1-1.95 \cdot 10^{-5}+3.31 \cdot 10^{-6} i+1-3.23 \cdot 10^{-6}+1.19 \cdot 10^{-9} i\right) / 2 \\
& =1-1.14 \cdot 10^{-5}-1.65 \cdot 10^{-6} i \\
\delta_{\mathrm{avg}} & \approx 1.14 \cdot 10^{-5} .
\end{aligned}
$$

At a grazing angle of $\theta=10 \mathrm{mrad}$, the simple and modified Bragg equation result in layer spacings of

$$
\Lambda^{\mathrm{B}}=\frac{\lambda}{2 \sin \theta} \approx 5 \mathrm{~nm}, \quad \Lambda^{\mathrm{mB}}=\frac{\lambda}{2 \sqrt{n^{2}-\cos ^{2} \theta}} \approx 5.688 \mathrm{~nm},
$$

which is almost $14 \%$ larger. A very large effect in view of the rather small decrement $\delta_{\text {avg }}=1.14 \cdot 10^{-5}$. 
Application to elliptical multilayers Now let us find a distortion function $\varphi(s, t)$ that describes this modification which is needed to correct for refraction and propagation. Using the definitions of Bragg's law and the modified Bragg condition, the ratio $R$ of the layer spacings $\Lambda^{\mathrm{mB}}$ and $\Lambda^{\mathrm{B}}$ is

$$
R:=\Lambda^{\mathrm{mB}} / \Lambda^{\mathrm{B}}=\frac{\sin \theta}{\sqrt{n^{2}-\cos ^{2} \theta}} \approx \frac{\sin \theta}{\sqrt{(1-2 \delta)-1+\sin ^{2} \theta}}=\left(1-2 \frac{\delta}{\sin ^{2} \theta}\right)^{-1 / 2} .
$$

A Taylor series expansion yields

$$
R=\sum_{n} R_{n} \approx 1+\frac{\delta}{\sin ^{2} \theta}+\frac{3}{2}\left(\frac{\delta}{\sin ^{2} \theta}\right)^{2}+\ldots
$$

this results in a stretching of the ML structure. In accordance with subsection 4.3.1, the transformation of the $t$-coordinate may be described by a distortion function $\varphi(s, t)$. In the case of the modified Bragg condition, this turns out to have the form

$$
\varphi(s, t) \approx 2\left(t-t_{0}\right) \delta / \sin ^{2} \theta
$$

Comparing with (4.11), this expression can be interpreted as a "local rotation" of angle $\Delta \theta=\delta / \sin \theta(s, t)$. As a first approximation, we again neglect the fact that $\theta$ depends on coordinates; then the derivatives of $\varphi$ are

$$
\varphi_{, t}=2 \delta / \sin ^{2} \theta, \quad \varphi_{, t t}=\varphi_{, s}=\varphi_{, s s}=0 .
$$

The new expression for $\varepsilon$ is

$$
\varepsilon=\beta^{2}\left(-2 \varphi_{, t}+\varphi_{, t}^{2}\right)=-4 \beta^{2} \delta / \sin ^{2} \theta+4 \beta^{2} \delta^{2} / \sin ^{4} \theta \approx 2 \chi_{0},
$$

because $\chi_{0}=-2 \delta$ and $\beta^{2}=\sin ^{2} \theta$. But then $\chi_{0}^{\prime}=\chi_{0}-\varepsilon=-\chi_{0}$.

The Takagi-Taupin equations for nearly elliptical multilayer mirrors, fulfilling the modified Bragg condition, read

$$
\begin{aligned}
\left(\alpha^{2} \partial_{s}+\beta^{2} \partial_{t}\right) \psi_{0} & =i\left(+u_{0} \psi_{0}+u_{\overline{1}} \psi_{1}\right)-\gamma^{+} \psi_{0}, \\
\left(\alpha^{\prime 2} \partial_{s}-\beta^{\prime 2} \partial_{t}\right) \psi_{1} & =i\left(-u_{0} \psi_{1}+u_{1} \psi_{0}\right)+\gamma^{\prime-} \psi_{1},
\end{aligned}
$$

with

$$
\alpha^{\prime 2}=\alpha^{2}, \quad \beta^{\prime 2}=\beta^{2}\left(1-2 \delta / \sin ^{2} \theta\right) .
$$

This change of sign in $u_{0}$ for the reflected wave can be interpreted as follows: The incoming beam suffers phase shift upon propagation; thus for "ideal" ellipses the Bragg condition is not fulfilled exactly. By changing the layer spacing appropriately, the optical path difference is adjusted to resonance. 
In other, more abstract, words, we allow the incoming beam $\psi_{0}$ to accumulate phase shift, but this is corrected by an "anti-phase shift" of the reflected beam, $\psi_{1}$.

In (4.17), the directional derivative is corrected by the factor $1-2 \delta / \sin ^{2} \theta$. For a $W / B_{4} C-$ $\mathrm{ML}$ at a photon energy of $12.4 \mathrm{keV}$ and an angle of incidence of $10 \mathrm{mrad}$, this correction is about $1-0.23$ and thus significant. This correction represents the fact that the deformed layer reflects the beam in a different direction.

The anti-phase shift reflected in the sign of $u_{0}$ in (4.16) is not the ideal solution. Soon we will introduce a modification factor $f$ for numerical optimisation.

In the derivation of the modified Bragg condition, only the planar case has been considered; then, the modified layers are still parallel, preserving angles. In the elliptical case, the modified layer shapes reflect in a different direction. Since the local angle of rotation, $\delta / \sin \theta$, depends on coordinates, the once confocal ellipses are strained and are described by more complicated shapes in cartesian coordinates. But in the transformed coordinates $\left(s, t^{*}\right)=(s, t+\varphi(s, t)$, these non-conformal non-ellipses are described as straight lines.

Let us restate the approximations. The ratio $R$ of modified Bragg condition and Bragg's law is treated linearly in $\delta$ and quadratically in $\theta$. The distortion function $\varphi$ is correct as long as $\sin \theta \approx \theta$, see appendix D.3.2. The derivatives $\varphi_{, s}$ and $\varphi_{, t}$ have been stated assuming $\theta=$ const. The validity of this assumption is discussed briefly in appendix $\mathrm{H}$. For the final form of the TT equations, again terms of order $\delta^{2}$ have been neglected.

It has to be noted that the linear approximation of the layer thickness ratio $R \approx(1+$ $\left.\delta / \sin ^{2} \theta\right)$ is not valid if the higher-order terms starting with $R_{2}=\left(\delta / \sin ^{2} \theta\right)^{2}$ do contribute significantly. Since $\theta_{c} \approx \sqrt{2 \delta}$, this happens near the critical angle. But then the concept of $M L$ mirrors is not of interest.

Modification factor The modified Bragg condition (4.13) was derived from geometrical principles in planar MLs. Analytical theory gives confidence that it indeed is superior to Bragg's law, since it corrects for refraction. To make further optimisation possible, we now introduce an open parameter $f$ :

$$
\Lambda(f, s):=\Lambda^{\mathrm{B}}(s)+f \times \Delta \Lambda(s), \quad \Delta \Lambda(s)=\Lambda^{\mathrm{mB}}(s)-\Lambda^{\mathrm{B}}(s) .
$$

Obviously, for $f=0$ we have Bragg's law, whilst $f=1$ gives the modified Bragg condition. In between, $f$ interpolates linearly. An "anti-correction" can be modelled using negative values, while $f>1$ corresponds to "overcompensating". We will use this scaling factor $f$ later for numerical optimisation of focus properties. 
Example At a photon energy of $12.4 \mathrm{keV}$, a $\mathrm{ML}$ mirror made of $\mathrm{W} / \mathrm{B}_{4} \mathrm{C}$ has an average $\delta \sim 1.14 \cdot 10^{-5}$; at an angle of incidence of $\theta \sim 10 \mathrm{mrad}$ we get $R_{1} \sim 0.1, R_{2} \sim 0.01$. In addition, $\varepsilon$ has a contribution of $\varphi_{, t}^{2}$ likewise neglected so far. In the next subsection we will present more accurate calculations that will mainly confirm these first considerations.

The modification of $\beta^{\prime 2}=\beta^{2}\left(1-2 \delta / \sin ^{2} \theta\right)$ is on the order of a few percent for reasonable values. So the reflected wave propagates in a slightly different direction. This has to be the case since the "locally rotated" layers reflect in a different direction. But hence the geometrical path of the reflected beam changes, and it can be expected that the modified Bragg condition still might not be optimal. This can be anticipated for the modified Bragg condition has been derived in planar geometry; but in the elliptical case, the modified layers are no longer confocal ellipses.

\subsection{Solving the Takagi-Taupin equations}

In the previous sections we have derived a system of coupled differential equations, (4.7), following the Takagi-Taupin formalism, for ML mirrors constructed from confocal ellipses. This approach has then been generalised for optimised shapes correcting for refraction (subsection 4.3.2). As shown in (4.16), the modified Bragg condition (4.13) results in a sign change of the propagation term $u_{0}$, interpreted as an "anti-phase shift". After these analytical considerations we now seek for numerical techniques to integrate (4.7), to gain more quantitative insights.

In the following subsections, we first consider the boundary conditions (subsection 4.4.1) imposed by the physical set-up, develop a numerical scheme for the direct integration (subsection 4.4.2), and discuss assumptions and limitations of this model (subsection 4.4.3). Closing this section, we summarise applied tests to the implementation. Comparison with analytical results is given in section 4.5 .

\subsubsection{Boundary conditions}

Actual solutions of the Takagi-Taupin system of equations (4.7) depend on boundary conditions, which are given by the incoming radiation field. In elliptical coordinates, the $\mathrm{ML}$ structure is given as a "rectangular" region having four "flat" boundaries. So we need eight boundary values for the two wave fields $\psi_{0}$ and $\psi_{1}$. But only half of them are known; the other half is to be determined by the simulation. On the "left" boundary, both the incoming field $\psi_{0}$ and the reflected field $\psi_{1}$ vanish; furthermore, $\psi_{1}$ is zero along the "top" surface, assuming no reflection the vacuum or substrate. The incoming radiation field determines $\psi_{0}$ 
at the "bottom" layer. Here, $\psi_{1}$ is the wanted solution and hence not known. The "incoming" $\psi_{0}$ on the "top" surface corresponds to a transmitted beam that can be calculated by the simulation. In addition, both fields $\psi_{0,1}$ leave the structure through the "right" boundary; the field there is not of interest to us. See also figure 4.8 .

So the known boundary conditions are

- $\psi_{0,1}($ left $)=0$,

- $\psi_{1}($ top $)=0$,

- $\psi_{0}($ bottom $)=$ illumination; while the calculated field quantities are

- $\psi_{1}($ bottom $)=$ reflected field,

- $\psi_{0}($ top $)=$ transmitted field.

- $\psi_{0,1}($ right $)=$ not of interest,

The reflected field $\psi_{1}$ (bottom) then can be propagated to the focal region, using the mirror simulation from chapter 3 .

\subsubsection{Numerical methods}

Now that the boundary conditions for (4.7) are known, we need a numerical scheme for the actual integration of the system. To solve differential equations, two main methods exist. Finite difference (FD) schemes approximate infinitesimal derivatives by fractions with finite, but small grid spacing $h$. The grid spacing $h$ (in two dimensions $h_{s} \neq h_{t}$ in general) has to be small enough to give accurate approximations, but not too small that numerical errors dominate. Traditionally, this is the method of choice for solving Takagi-Taupin equations $[79-81,90,91]$. Usually, the grid parameters $h_{s}$ and $h_{t}$ are constant in the (two-dimensional) volume, although adaptive grids have been proposed [95]. In the present work constant $h_{t}=\Lambda^{\mathrm{B}} / 2$ and an appropriate constant $h_{s}$ are used; although this choice is not optimal for $M L$ mirrors with strong gradients.

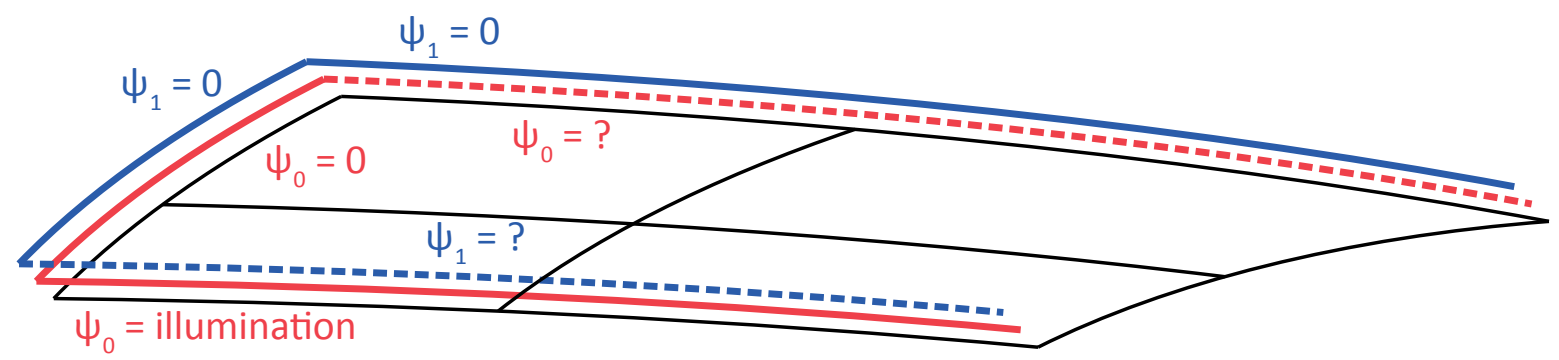

Figure 4.8: Boundary conditions for the incoming field $\psi_{0}$ and the reflected field $\psi_{1}$. Coloured lines are known, dashed lines are to be determined by the TT simulation. 
The second famous technique is the finite element method (FEM). The domain of interest is covered by a mesh of points; these points can be arranged nearly arbitrarily. The FEM thus allows for a local density of mesh points that is optimised for the geometry and function gradients. Although the FEM often is superior to FDs in arbitrary geometries, a solution scheme has not been found. Boundary conditions have to be given for all boundaries; since especially the values of the reflected amplitude at the "bottom" surface are not known, this is not directly possible. Instead, weak conditions determining the derivative of $\psi_{1}$ may be given; but a scheme to solve the TT equations could not be found within reasonable time.

System of coupled PDE The Takagi-Taupin system of coupled differential equations (4.7) we are about to solve is of the form

$$
\nabla_{0} \psi_{0}=c_{00} \psi_{0}+c_{01} \psi_{1}, \quad \nabla_{1} \psi_{1}=c_{11} \psi_{1}+c_{10} \psi_{0}
$$

in this symbolic notation, $\nabla_{0}$ and $\nabla_{1}$ represent the directional derivatives, and all coefficients are collected in the $c_{i j}$. Both $\nabla$ and $c$ depend on $(s, t)$. The known boundary values are

$$
\begin{aligned}
\psi_{0}(s=0) & =0, & \psi_{0}(t=0) & =\text { illumination, } \\
\psi_{1}(s=0) & =0, & \psi_{1}(t=\infty) & =0,
\end{aligned}
$$

denoting the "upper" boundary as $t=\infty$. Our a priori information thus is asymmetric: $\psi_{0}$ is known on the entrance surface, where $\psi_{1}$ is to be determined; $\psi_{1}$ is known on the substrate surface, where $\psi_{0}$ shall be calculated. This is a manifestation of the nature of Bragg reflection; in Laue geometry, both $\psi_{0,1}$ would be known on the entrance.

The usual approach in PDE solving expresses the differentials in (4.19) as finite differences on a regular grid. The equation is written as a matrix-vector product and solved for $\psi\left(s_{n+1}\right)$ by inverting the matrix. Efforts in implementing such a scheme for (4.19) in Bragg geometry did not succeed; therefore, a more naïve approach has been chosen, that directly propagates $\psi$ in $s$ and $t$ direction.

Solving Takagi-Taupin in Bragg geometry The "rectangular" domain (in elliptical coordinates) is discretised on a regular grid of spacings $\Delta s \times \Delta t$. Then the partial derivative $\nabla_{0} \psi_{0}$ can be approximated by

$$
\nabla_{0}^{\prime} \psi_{0}=\left(\partial_{s}+\partial_{t}\right) \psi_{0} \approx \frac{\Delta_{s} \psi_{0}}{\Delta s}+\frac{\Delta_{t} \psi_{0}}{\Delta t}
$$

(The factors $\alpha^{2}, \beta^{2}$ have not been written for sake of simplicity.) The differences $\Delta_{s} \psi_{0}$ and $\Delta_{t} \psi_{0}$ are given by forward and backward differences,

$$
\begin{array}{ll}
\Delta_{s} \psi_{0}=\psi_{0}\left(s_{n+1}\right)-\psi_{0}\left(s_{n}\right), & \Delta_{t} \psi_{0}=\psi_{0}\left(t_{n}\right)-\psi_{0}\left(t_{n-1}\right) \\
\Delta_{s} \psi_{1}=\psi_{1}\left(s_{n+1}\right)-\psi_{1}\left(s_{n}\right), & \Delta_{t} \psi_{1}=\psi_{1}\left(t_{n+1}\right)-\psi_{1}\left(t_{n}\right)
\end{array}
$$


The four-point stencil used is shown in appendix D.2, figure D.1 (b). Also a five-point stencil using a central difference approximation for $\Delta_{t}$ - as shown in figure D.1 (c) - was implemented, but it had to be discarded since energy was not conserved, see the discussion in appendix D.2.

Grid size In pure mathematics, (4.20) becomes an equality in the limit $\Delta s, \Delta t \rightarrow 0$. But in numerical mathematics, this limit cannot be made: this would require infinite amount of time and memory. Finite representation of real numbers with a precision of 53 bit $^{\S}$, a relative precision of roughly $10^{-16}$ is achieved. It is common practice that the differences in the approximation of (4.20) should not be smaller than $10^{-8}$, setting a lower bound for the approximation. An upper bound usually has to be found by trial and error: the grid spacings $\Delta s, \Delta t$ are increased up to values where the "solution" is obviously invalid, typically diverging.

It has been found that for reasonable grid spacings the number of discretisation points in the $t$-direction should be about twice the number of bilayers. For the ratio of the grid spacings, $\Delta s / \Delta t$, the following gridratio has shown to be of use:

$$
\mathrm{GR}=\operatorname{gridratio}:=\theta \times \Delta s / \Delta t
$$

with the gracing angle of incidence $\theta$ at the centre of the mirror. Tests have shown that a value of $\mathrm{GR} \lesssim 100$ should be used; for ML mirrors with a strong gradient of the local angle of incidence GR $\lesssim 10$ may be needed. This is due to the fact that the local angle of incidence enters (4.7) in the factor $\alpha^{2}$ and $\beta^{2}$.

Computational complexity The computational complexity is directly proportional to the number of grid points in both directions. The number of points along $t$, $\# T$, is suggested to be twice the number of layers \# $L$; with $\# S$, the number of points along $s$, proportional to $\theta$ at constant GR, and to the length $L$ of the mirror, the overall complexity $C$ can be expected to be

$$
C=\# S \# T \propto L \cdot \# L \cdot \theta(s=\infty) ;
$$

with $s=\infty$ denoting the value of the $s$-coordinate at the "right" edge of the ML mirror, so $\theta(s=\infty)$ is just the maximum local angle of incidence.

The memory consumption $M$ of the current implementation is roughly

$$
\begin{array}{r}
M \sim m_{1} \# S \# T+m_{2} \# S+m_{3} \# T ; \\
m_{1}=16 \mathrm{~B}, \quad m_{2}=40 \mathrm{~B}, \quad m_{3}=120 \mathrm{~B} .
\end{array}
$$

$\S$ This is the effective mantissa size of an IEEE 754 double precision variable usually used in present day's computers. The actual mantissa in normalised form stores 52 bit, where a leading 1 is omitted. 
To store the propagated field envelopes $\psi_{0}$ and $\psi_{1}$, memory of size as given by the first term is needed. The envelopes can be written to file. The second term is needed to store the illumination and the reflected amplitudes; furthermore, several arrays of size \#S are used to hold pre-calculated values of the local angle of incidence. The third term, usually the smallest, is given by the need to store the previous and current slices of $\psi\left(s_{n}\right), \psi\left(s_{n+1}\right)$, plus pre-calculated values like $\alpha^{2}$ and $\beta^{2}$.

Via the command line argument -- maxmem=<value $\rangle$, a memory limit in megabytes may be activated; virtually all memory allocations then are checked to respect this limit prior to the call of malloc; in addition, a limit using the setrlimit system call is set.

A small GR implies a small $\Delta s$ and hence a large number of discretisation points; typically, a high density along $s$ is only needed where the local angle of incidence is large. So a lot of computational resources are wasted on the regular grid with constant $\Delta s$. The results shown further below have been calculated on the ten second- to minutes scale per $\mathrm{ML}$ simulation. It might be wise to implement a local grid spacing $\Delta s(s)$. This would, however, be a formidable task of programming.

\subsubsection{Limitations and model assumptions}

In this subsection we describe several limitations, assumptions, and open questions concerning the "correctness" of the described theory and its implementation into numerical code.

\section{Finite differences, numerical precision}

Whilst analytical expressions are "correct" (usually within some approximations of the underlying theory), numerical solutions suffer from finite precision, commonly referred to as rounding or truncation errors. Within this work, care has to be taken to use reasonable grid spacings $\Delta s$ and $\Delta t$. Grid sizes of $\Delta t \sim \Lambda / 2$ are suggested, although this has not been tested thoroughly.

To estimate errors, the TT theory can be compared with analytical results like Parratt's algorithm, cf. section 4.5. The latter describes reflectivity of plane waves at planar ML mirrors of infinite length. If a parameter is scanned for optimisations, this should be done for fixed $\Delta s$ and $\Delta t$, so the approximate "incorrectness" remains comparable. 


\section{Scalar wave theory}

Within this work, we do not deal with the vector equations first given by Maxwell, but only with the scalar wave equation (4.3); this is a common simplification of the TakagiTaupin theory [92]; a derivation of TT equations for ML Laue lenses has been given in [43]. At typical geometries considered here, gracing angle of incidence is small compared to the Brewster angle; then polarisation effects can be neglected. This is, however, under discussion [99].

\section{Two-beam approximation}

Only considering one incoming and one reflected beam is highly encouraged by a priori knowledge, focusing by an elliptical mirror. Nevertheless, higher order diffraction is possible due to neglected terms in the Fourier expansion of the susceptibility.

\section{Interface to vacuum}

The boundary conditions of the incoming beam, $\psi_{0}$ at the surface, are somehow artificial. For simplicity, we just assume that the fields on the inside of the surface are given by the illumination on the vacuum side. However, a correction due to the Fresnel coefficient of transmission would be appropriate. But that coefficient depends on the index of refraction - and it is not clear if the first mono-layer or an averaged value of the bilayer would be the right choice.

In addition to the Fresnel coefficient of transmission (and a correction term of the "refracted Poynting vector"), one could also argue that one additional Fresnel reflection at the vacuum-first layer interface should be included separately; but this contribution would be rather small for $\theta \gg \theta_{c}$. Also the coefficient of transmission is expected to be nearly unity.

\section{Idealised structure}

Within this thesis, the ML structure is assumed to be "perfect", we assume that the index of refraction is given by a square wave, although approximated by a first order Fourier expansion, hence sine wave. In reality, roughness is present at the interfaces, diffusion and chemical reactions take place. The deposition process introduces another source of deviations; the actual thickness of a layer is randomly distributed, maybe correlated to neighboured layers. Some of these real-structure effects may be included using the Takagi-Taupin formalism by 
introducing additional phase factors to describe deviations from the design profile (like the scaled modified Bragg condition).

It is desirable to model distorted layers using some measured deviation profile that describes an appropriate modification function $\varphi(s, t)$ as introduced in subsection 4.2.2. However, its derivatives $\varphi_{, s}$ and $\varphi_{, t}$ are needed in (4.9). It can be expected that the deviation profile has to be filtered before finite differences approximate these derivatives.

As a first approximation the following model might be used: The height deviation profile is projected onto the surface and used as a modulation of the illumination function. This distorted field is propagated through the ideal structure, and the reflected field is modulated, too.

\section{Tabulated optical constants}

The susceptibility $\chi$ of the two layer materials is obtained from tabulated values of the optical index $n$. It is questionable if these values are appropriate for very thin films of $\Lambda / 2 \sim 1 \mathrm{~nm}$. Furthermore, the deposition process and its parameters (e.g. gas pressure, voltage of the anodes, etc.) show influence on the optical properties. It is common practice to scale the optical parameters to obtain a best fit of Parratt-like calculations with measured rocking curves [100]. Therefore, command line arguments -- factordelta $=\langle\mathrm{value}\rangle$ and - - factorbet $a=<$ value $>$ have been implemented.

\section{Energy conservation}

In appendix D.2 we summarise that using a central difference approximation of the partial derivatives violates energy conservation. The current implementation gives more reasonable results, although due to numerical problems energy may not be conserved (apart from absorption, which can be "disabled" using the command line argument --factorbeta=0.). If such problems occur, it might be helpful to use the following correction method:

1. calculate a single propagation step including absorption,

2. calculate the same propagation step with absorption disabled;

3. from the latter, obtain a correction factor for the intensities,

4. and apply it to the fields $\psi_{0,1}$ propagated with absorption. 


\section{Very few layers}

The chosen formalism does not describe reflection at discrete interfaces within the ML structure, but treats the whole volume as one diffracting entity; thus it is possible to simulate a Bragg reflection of an infinitely thin volume - this is obviously nonsense. The applicability of this continuum model is questionable in case of few layers.

\subsubsection{Tests of the implementation}

We report very briefly about tests of the implementation that have been carried out. These tests have been performed to make sure that the integration scheme for (4.7) is correct. Regarding physical correctness, see the following section 4.5, where Takagi-Taupin results are compared with Parratt's algorithm.

- Propagation of $\psi_{0}$ : this has been tested setting the coupling $u_{ \pm 1}=0$ and applying the usual boundary conditions.

- propagation of $\psi_{1}$ : again, we set $u_{ \pm 1}=0$, but set $\psi_{0}=0$ on the entrance surface, $\psi_{1}=1$ on the substrate surface.

- Coupling $\psi_{0} \rightarrow \psi_{1}$ : setting $u_{1}=0$, no back-refraction occurs. Energy is exchanged from the incoming wave field to the reflected wave.

- Coupling $\psi_{1} \rightarrow \psi_{0}: u_{\overline{1}}=0$, while illuminating the substrate surface and $\psi_{1}$.
- Illumination of the entrance surface with Gaussian envelopes of different widths, testing for anomalies like non-linearities.

- Testing for energy conservation. The stencil for the finite differences had to be adjusted, see appendix D.2.

- Searching for reasonable grid spacings $\Delta s$ and $\Delta t$ : the latter was decreased until "convergence" was achieved, while $\Delta s$ was increased until the integration scheme diverges. Q.v. (4.21) and the discussion there.

\subsection{Integrated reflectivity}

In this section we calculate the integrated reflectivity, i.e.

$$
R=\frac{\int_{\text {surface }}\left|\psi_{1}(s)\right|^{2} \mathrm{~d} s}{\int_{\text {surface }}\left|\psi_{0}(s)\right|^{2} \mathrm{ds}}
$$

for different set-ups. This allows for a quick characterisation of the reflectivity performance. Different parameters can be changed, like the mirror length $L$, the number of layers $N$, or the layer shape. In the following we will have a closer look at $R(\Delta \theta)$ : This is basically the reflectivity curve while changing the actual angle of incidence. As stated earlier, in the Takagi-Taupin approach of curved ML mirrors, the angle $\Delta \theta$ is a parameter for the 
deformation of the ML structure. This parameter allows for comparison to well-established techniques known from the theory of flat crystals.

The next subsections present comparisons in terms of $R(\Delta \theta)$ obtained from Parratt's algorithm and our Takagi-Taupin approach; while Parratt uses a flat structure of infinite length, the computations with Takagi-Taupin have been carried out for flat multilayers, elliptically shaped multilayers (Bragg condition), and optimised multilayers (modified Bragg condition) of finite lengths.

The geometrical parameters of the test systems are shown in table 4.1. Photon energy is $12.4 \mathrm{keV}$ in most cases; some simulations have been carried out with $24.8 \mathrm{keV}$ and $49.6 \mathrm{keV}$.

\begin{tabular}{rlrl} 
Quantity & Value & Quantity & Value \\
\hline distance from source $^{\dagger}$ & $50.0 \mathrm{~m}$ & number of bilayers & $20,50,200$ \\
distance to focus ${ }^{\dagger}$ & $0.1 \mathrm{~m}$ & material 1 & $\mathrm{~W}$ \\
length of mirror & $4.0 \ldots 120 \mathrm{~mm}$ & material 2 & $\mathrm{~B}_{4} \mathrm{C}$ \\
angle at mirror's centre & $10.0 \mathrm{mrad}$ & $\Gamma$-ratio & 0.5
\end{tabular}

Table 4.1: Geometrical parameters of the test systems simulated throughout this section. Parameters marked with a dagger $\left({ }^{\dagger}\right)$ are not applicable in case of flat ML. The Fourier coefficients of the susceptibility $\chi$ can be found in appendix $I$.

\subsubsection{Flat multilayers}

We compare the reflectivity curve $R(\Delta \theta)$ for flat multilayers, as obtained by Parratt's algorithm and using the Takagi-Taupin formalism.

Parratt's algorithm Parratt's iterative approach for flat multilayers models infinitely long layers by their Fresnel coefficients of transmission and reflectivity for illumination with a plane wave under a given angle $\theta$. If the Fresnel reflectivity of the surface between layers $i$ and $j$ is $r_{i j}$, the net reflection $r_{i}$ at layer $i=j-1$ is given by $[52,54]$

$$
r_{i}=\frac{r_{i j}+r_{j} \exp \left(2 i \beta_{i}\right)}{1+r_{i j} r_{j} \exp \left(2 i \beta_{i}\right)},
$$

$r_{j}$ being the net reflection at layer $j$ and $\beta_{i}=2 \pi d_{i} n_{i} \cos \theta_{i} / \lambda$ the optical thickness of the $i$-th layer with thickness $d_{i}$, optical index $n_{i}$, and refracted angle of incidence $\theta_{i}$. The wellestablished IMD software by David Windt has been used for our comparison calculations [52]. 
Takagi-Taupin for flat multilayers In our derivation of the Takagi-Taupin equations we have assumed cylindrical waves and elliptically shaped layers; for the flat ML case with plane waves, we simply set all coefficients $\alpha$ and $\beta$ constant to their value at the centre of the respective curved MLs. Illuminating with plane waves, the terms $\gamma^{ \pm}$do not contribute. The simulation code comes with a command line argument - $-\mathrm{fl}$ at that uses these specialisation. Reflectivity curves can be obtained by scanning the parameter --rot. theta, see appendix D.3.1.

Simulation Exemplary plots comparing Parratt's algorithm as implemented in IMD [52] and the Takagi-Taupin implementation developed for this thesis are shown in figure 4.9. In the top row, the photon energy has been set to $12.4 \mathrm{keV}$, while the bottom row shows calculations at $49.6 \mathrm{keV}$. Left plots are for MLs with 20 layers, the plots on the right hand side with 50 layers. The nominal Bragg angle is $\theta_{\mathrm{B}}=10 \mathrm{mrad} \approx 0.57^{\circ}$ in all cases. Whilst Parratt's algorithm assumes an infinite mirror length, the TT simulation has been carried out for a mirror length of $L=4 \mathrm{~mm}$. The number of discretisation points in $t$-direction (into the ML structure) has been set equal to the number of layers; see appendix D.4 for a short summary of studies concerned with grid discretisation parameters.

Comparison According to these and similar calculations, Parratt's algorithm (blue dotted lines) predicts higher reflectivities below, and slightly smaller reflectivities above the Bragg peak, than the TT simulation (red lines). Both methods show characteristic oscillations below the Bragg peak for few layers, that are known from experiments. All in all, both theories give similar results. Since they are based on different model assumptions, small differences are expected.

\subsubsection{Curved multilayers (Bragg type)}

In this subsection we compare reflectivity curves $R(\Delta \theta)$ for elliptically shaped multilayers (eML), viz., according to Bragg's law, with flat multilayers (fML) (also according to Bragg's law, but for plane waves), as described in the previous subsection.

Simulation The simulations shown in figure 4.10 have been obtained for the same parameters as those in figure 4.9. The red lines are the ones from figure 4.9, hence Takagi-Taupin solutions for a flat mirror of length $L=4 \mathrm{~mm}$. All other lines have been obtained for elliptical ML mirrors. Blue dashed curve is for $L=4 \mathrm{~mm}$, green dots for $L=40 \mathrm{~mm}$, and the thin orange line is calculated for $L=120 \mathrm{~mm}$. 


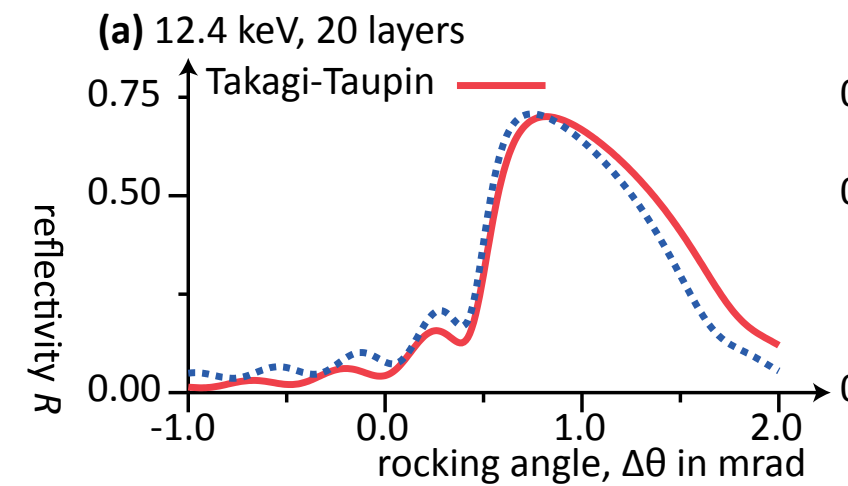

(b) $12.4 \mathrm{keV}, 50$ layers
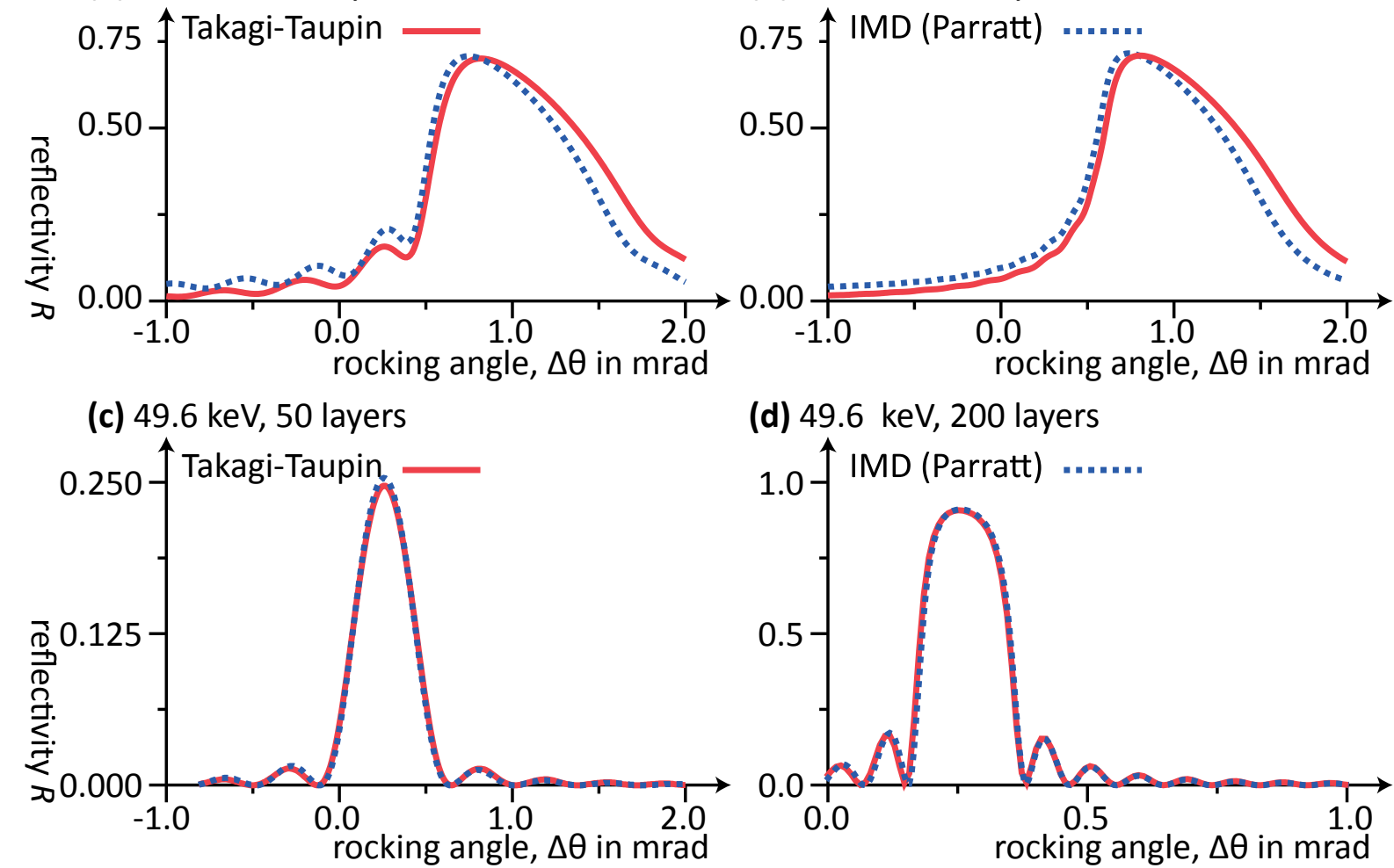

(d) $49.6 \mathrm{keV}, 200$ layers

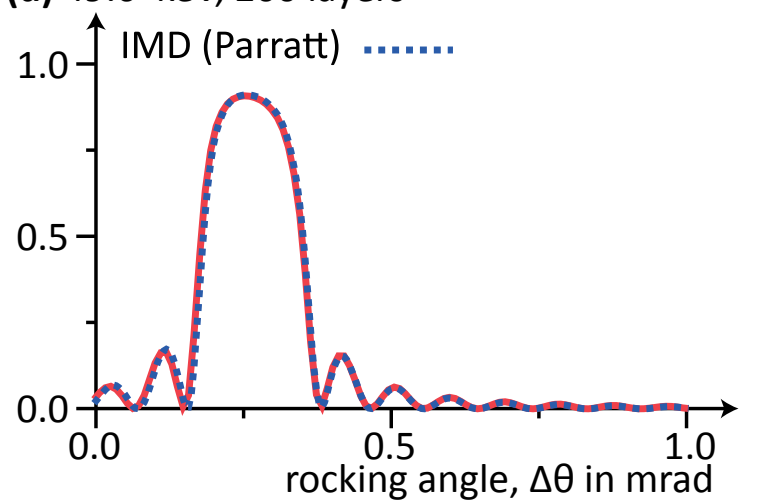

Figure 4.9: Comparison of Takagi-Taupin (red line) and Parratt's algorithm (IMD [52], blue dotted line), for indicated energies and layer numbers. Note the different axis scales. In the TT simulation, the mirror length has been set to $4 \mathrm{~mm}$, while the Parratt algorithm assumes an infinitely long mirror. The Bragg angle is $\theta_{\mathrm{B}}=10 \mathrm{mrad}$. Optical constants are for a $\mathrm{W} / \mathrm{B}_{4} \mathrm{C}-\mathrm{ML}$ mirror.

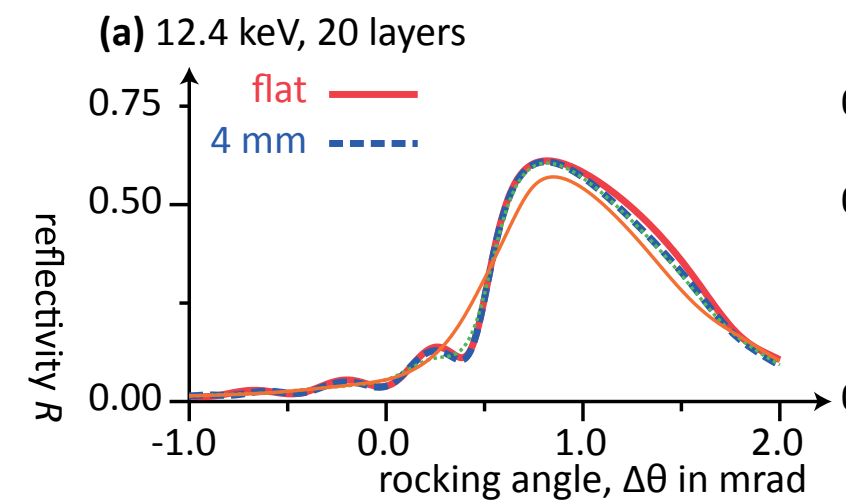

(b) $12.4 \mathrm{keV}, 50$ layers

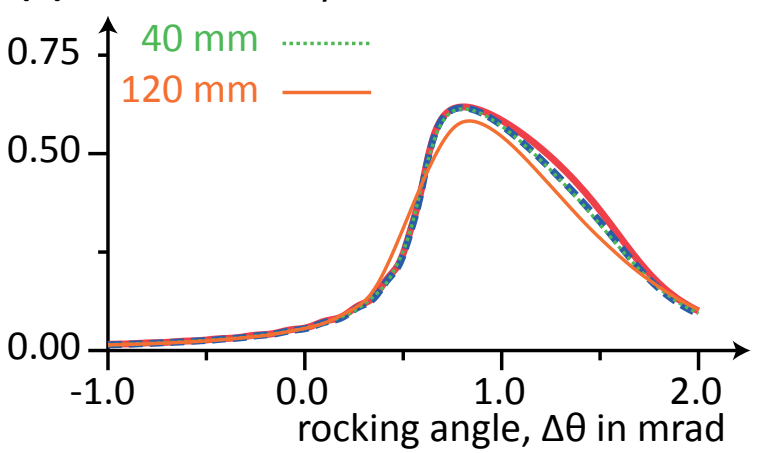

Figure 4.10: Comparison of reflectivity curves of elliptical ML mirrors of different lengths, obtained with the Takagi-Taupin formalism. Parameters are the same as in figure $4.9(a, b)$. Red lines are calculated for the flat case and a mirror length of $4 \mathrm{~mm}$. Curved mirrors have lengths of $4 \mathrm{~mm}$ (blue dashed), $40 \mathrm{~mm}$ (green dotted), and $120 \mathrm{~mm}$ (orange, thin). 


\begin{aligned} Quantity & Value & Quantity & Value \\ \hline distance from source & $50.0 \mathrm{~m} &$ number of bilayers & 100 \\ distance to focus & $10.0 \mathrm{~mm} &$ material 1 & $\mathrm{W} \\$ length of mirror & $10.0 \mathrm{~mm} &$ material 2 & $\mathrm{~B}_{4} \mathrm{C} \\$ angle at mirror's centre & $10.0 \mathrm{mrad} & \Gamma$-ratio & 0.5 \\ photon energy & $49.6 \mathrm{keV} &$ angular spectrum & $8.2 \ldots 14.1 \mathrm{mrad}\end{aligned}$

Table 4.2: Geometrical parameters for the simulation shown in figure 4.11.

Comparison Reflectivity curves of the short $L=4 \mathrm{~mm}$ mirror are nearly the same as in the flat case; the curvature is negligible. In case of the medium $L=40 \mathrm{~mm}$ mirror, the oscillations below the Bragg peak begin to wash out. Here the local angle of incidence varies significantly along the mirror's surface; the reflectivity for "one angle" (namely at the centre of the mirror) is an average of those for a large angular spread. For the long $L=120 \mathrm{~mm}$ mirror, hardly any oscillations are visible.

Reflected wave The intensity of the reflected wave inside the ML structure is shown in figure 4.11, for two rocking angles $\Delta \theta=\{-50,400\} \mu$ rad and the geometrical parameters given in table 4.2. The intensity maxima correspond to the Pendellösung effect known from dynamical diffraction. Here they follow the elliptical shape of the layers, since the Pendellösung periodicity depends on the local angle of incidence. This varies from about $8.2 \mathrm{mrad}$ on the left side to $14.1 \mathrm{mrad}$ on the right.

\subsubsection{Optimised curved multilayers (modified Bragg's law)}

Due to refraction, the Bragg peak occurs at a slightly different angle than expected. Using the modified Bragg condition (4.13) $[46,47,83]$, the individual layers in an optimised ML structure are not elliptic any longer. The derivation of (4.13) is based on ray optics and planar geometry; as discussed earlier, (4.16) supports this derivation in first order based on wave optics. For numerical optimisation, an open parameter $f$ has been introduced in (4.18). Here we sketch briefly how such an optimisation may look like.

Simulation As a model system the pseudo-rocking curve of the same ML mirrors as in figure 4.10 has been simulated. We call this a pseudo-rocking curve since not the deviation angle $\Delta \theta$, but the modification factor $f$ is scanned. The resulting reflectivity curve shows similar features, since the refraction correction can be regarded as a local rotation, q.v. (4.14). 


\section{Reflected wave inside ML mirror}

(a) $\Delta \theta=-50 \mu \mathrm{rad}$

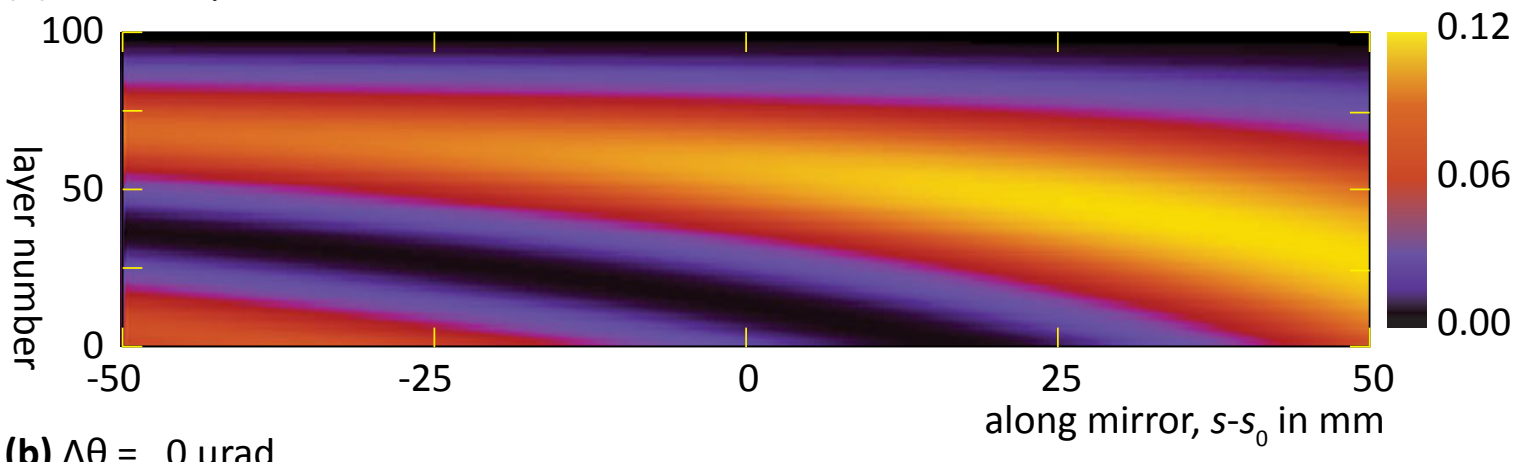

(b) $\Delta \theta=0 \mu \mathrm{rad}$

along mirror, $s-s_{0}$ in $\mathrm{mm}$

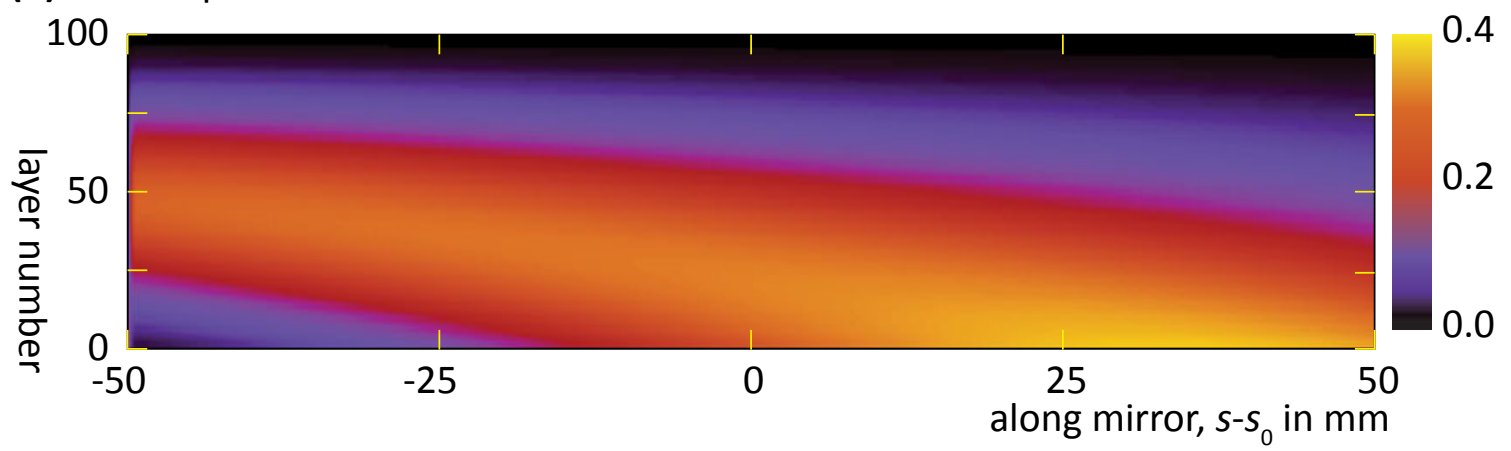

(c) $\Delta \theta=400 \mu \mathrm{rad}$

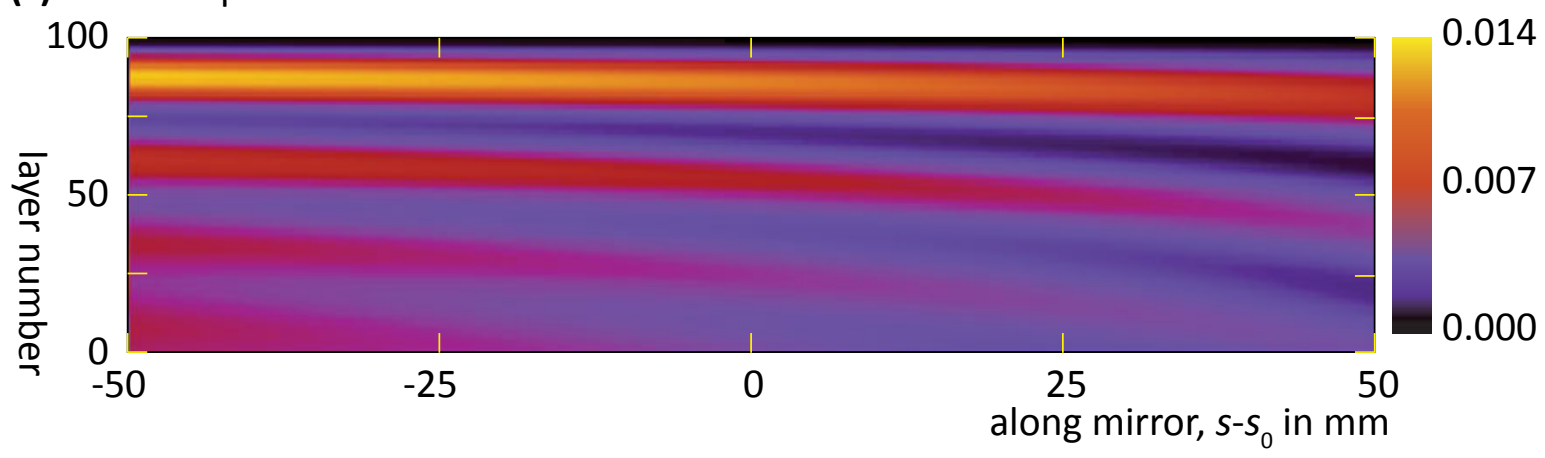

Figure 4.11: Intensity of reflected wave inside the multilayer, simulated for the parameters of table 4.2. Rocking angle $\Delta \theta$ is (a) $-50 \mu \mathrm{rad}$ and (b) $400 \mu \mathrm{rad}$. 


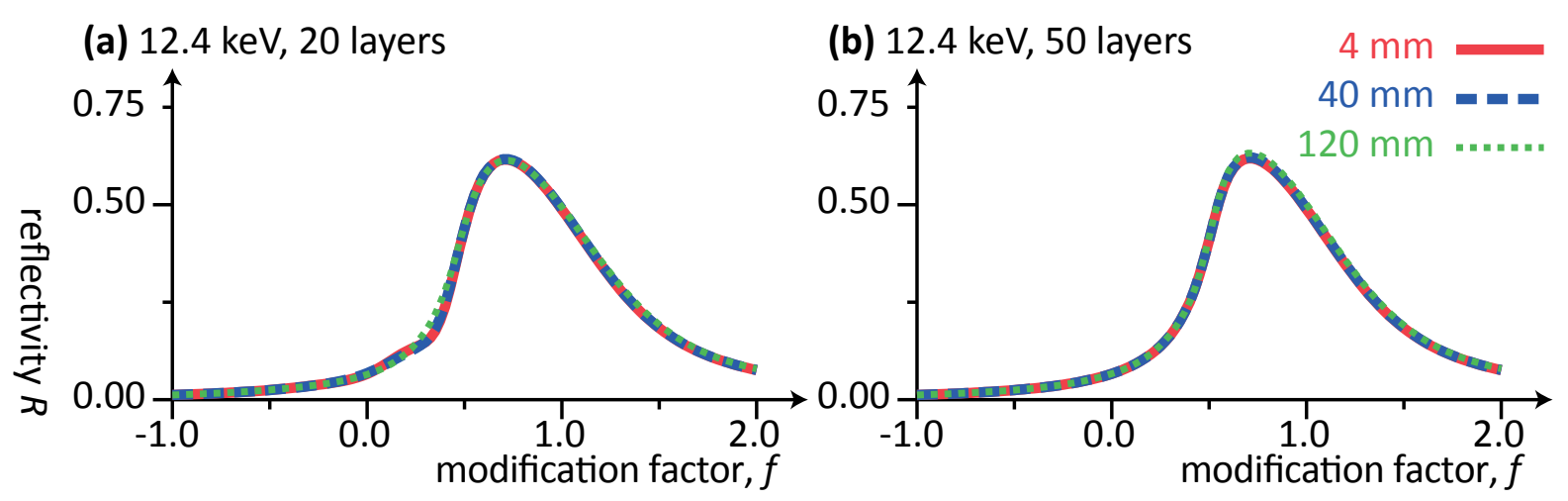

Figure 4.12: "Modified rocking curve": simulated reflectivity of curved multilayer mirrors, built using the modified Bragg condition (4.13), as a function of modification factor $f$, see (4.18). For this particular choice of parameters (given in table 4.1), mirror's length hardly influences the reflectivity.

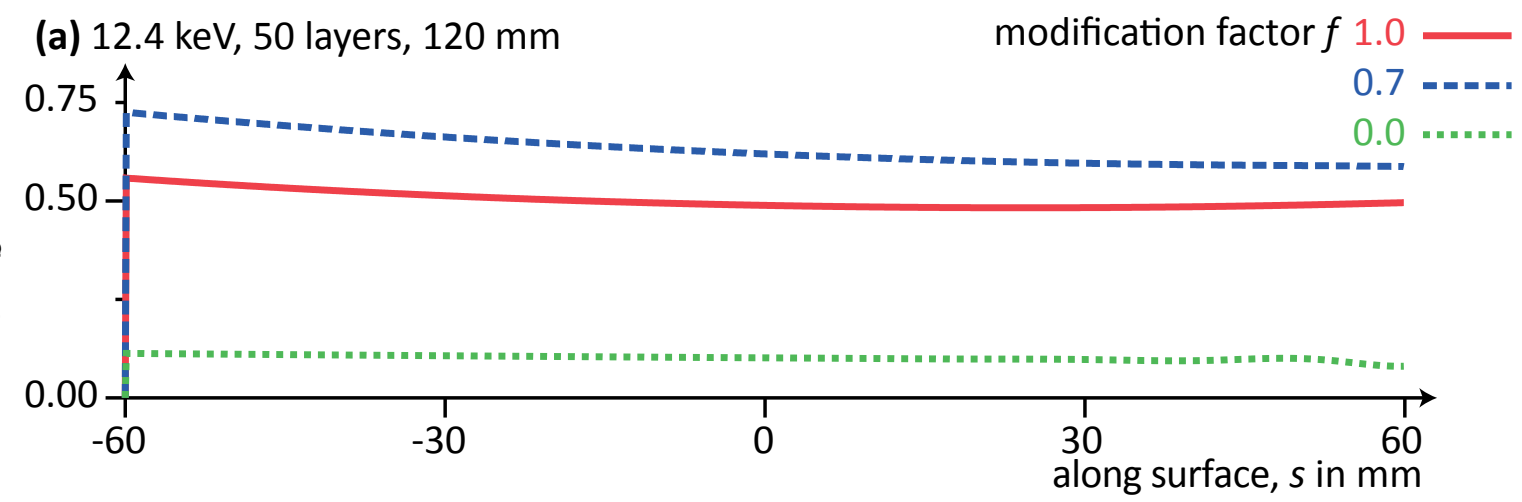

Figure 4.13: Reflectivity along multilayer surface for different modification factors $f=\{0.0,0.71 .0\}$ (green dots, blue dashes, red line). As a model system, the $L=120 \mathrm{~mm} \mathrm{ML}$ of figure 4.12 (b) with 50 layers has been chosen.

(a) $12.4 \mathrm{keV}, 50$ layers, $120 \mathrm{~mm}$ modification factor $f 1.0$

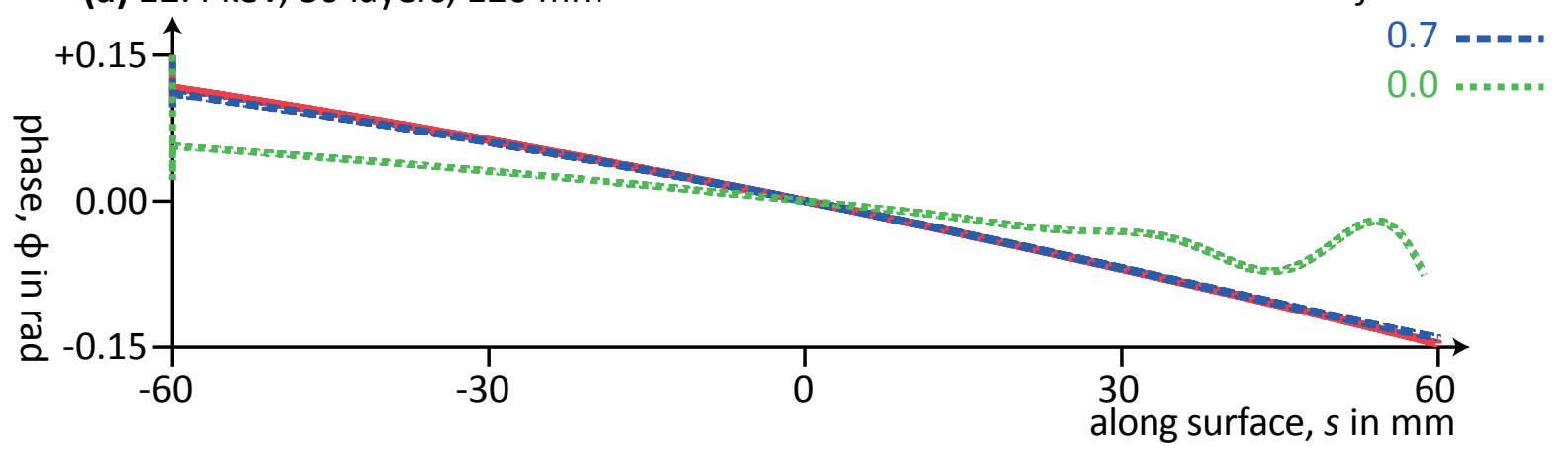

Figure 4.14: Reflected phase along multilayer surface, for the same set-up as in figure 4.13. The phase at the mirror's centre has been set to zero for clarity. 
Comparison Opposed to figure 4.10, the influence of the mirror length $L$ on the reflectivity curve of figure 4.12, simulated following the modified Bragg condition (4.13), is strongly reduced. The $L=120 \mathrm{~mm}$ mirror of figure 4.10, thin orange line, shows reduced reflectivity, since only the very centre fulfils the Bragg condition at a specific angle $\Delta \theta$. Using (4.13), the reflectivity is nearly independent of $L$. The oscillations below the "Bragg peak" have vanished.

Reflectivity and reflected phase along the mirror's surface are plotted in figure 4.13 and 4.14, for three different modification factors $f=\{1.0,0.7,0.0\}$ (red line, blue dashes, green dots), for a $L=120 \mathrm{~mm}-\mathrm{ML}$ with 50 bilayers - corresponding to the green dotted line of figure 4.12 (b). The reflectivity curves of full correction and "optimised correction", $f=\{0.7,1.0\}$, are nearly flat, despite some falling-off along the surface. This can be attributed to the fact that towards the "right" side of the $M L$, local angle of incidence increases recuding reflectivity. But in case of the "uncorrected" $M L$ (green dots, $f=0.0$ ), some waviness occurs on the "right". This is because the ML's surface cuts through the oscillations that are visible below the Bragg peaks in figures 4.9 and 4.10. Looking at 4.14, the reflected phase gets distorted, too.

\subsection{Results}

In this section, results of the presented multilayer simulation scheme are shown, covering both reflectivity and phase at the ML surface as well as focus properties. First we show results dealing with actual ML mirrors as used at the ID22NI beamline at the ESRF (subsection 4.6.1), and design parameters envisaged for a future ML KB system to be used at UPBL04 (subsection 4.6.2). In subsection 4.6.3, the physical limits of curved ML mirrors focusing are explored using parameters posing a challenge to current fabrication techniques. All results presented below are calculated for a $\mathrm{W} / \mathrm{B}_{4} \mathrm{C}$-multilayer of layer thickness ration $\Gamma=0.5$; optical constants have been taken from tabulations of bulk material [52]. See table 4.3 for geometrical parameters.

\subsubsection{ID22NI}

As first practical results simulate focusing properties of a Kirkpatrick-Baez (KB) ML mirror set-up, at present used for nano-focusing at the undulator beamline ID22NI at the ESRF has been simulated. The actual mirrors for horizontal (ML-ID22NIh) and vertical (ML-ID22NIV) focusing are adaptive mirrors on a bender, as shown in figure 4.15. The curvature of flexible

\footnotetext{
IUpgrade Programme Beamline 04
} 
mirrors can be adapted to current experimental parameters: depending on wavelength $\lambda$, the angle $\theta$ and curvature can be adjusted [101]. Source distances for the horizontally and vertically focusing mirrors differ. At the ID22NI, a horizontal slit at $27 \mathrm{~m}$ downstream from the undulator can be closed, yielding a "virtual source".

For sake of clarity, we show results calculated for only one photon energy of $E=17.5 \mathrm{keV}$ or correspondingly a wavelength of $\lambda=0.0709 \mathrm{~nm}$; the respective angles of incidence at the two mirrors' centre are then $\theta=8 \mathrm{mrad}$. For further geometrical parameters, see table 4.3. The results obtained for these two set-ups are shown in the following figures.

\begin{tabular}{r|cc|cc} 
& \multicolumn{2}{|c|}{ ID22NI } & \multicolumn{2}{c}{ UPBL04 (planned) } \\
Quantity & HFM & VFM & HFM & VFM \\
\hline distance from source & $36 \mathrm{~m}$ & $63 \mathrm{~m}$ & $144.6 \mathrm{~m}$ & $183.5 \mathrm{~m}$ \\
distance to focus & $83 \mathrm{~mm}$ & $180 \mathrm{~mm}$ & $43 \mathrm{~mm}$ & $100 \mathrm{~mm}$ \\
length of the mirror & $76 \mathrm{~mm}$ & $112 \mathrm{~mm}$ & $36 \mathrm{~mm}$ & $70 \mathrm{~mm}$ \\
angle at mirror's centre & $8 \mathrm{mrad}$ & $8 \mathrm{mrad}$ & $14.4 \mathrm{mrad}$ & $14.4 \mathrm{mrad}$ \\
$\Lambda_{1}$ & $5.35 \mathrm{~nm}$ & $5.07 \mathrm{~nm}$ & $2.93 \mathrm{~nm}$ & $2.86 \mathrm{~nm}$ \\
$\Lambda_{2}$ & $3.27 \mathrm{~nm}$ & $3.68 \mathrm{~nm}$ & $1.88 \mathrm{~nm}$ & $1.99 \mathrm{~nm}$
\end{tabular}

Table 4.3: Overview of the geometrical parameters for the current ID22NI beamline at the ESRF and a planned set-up within the ESRF upgrade programme. The angle of incidence can be changed to fit the current wavelength; the values here are stated for a photon energy of $E=17.5 \mathrm{keV}$ used for the shown simulations. Bilayer thicknesses $\Lambda_{1,2}$ are extreme values along the surfaces, assuming Bragg's law. Number of bilayers is 25 for all set-ups.

\section{Focus sizes}

In figure 4.16, simulated focus sizes for the horizontal (a) and vertical (b) mirror are shown, as a function of Bragg modification factor $f$, q.v. (4.18). The red curve shows the full width at half maximum of a $\operatorname{sinc}^{2}$-fit ( $c f$. the following Discussion) to the propagated intensity; the reddish error band indicates the error interval of the fit. The green dashed line is the standard deviation of the reflected phase envelope $\psi_{1}$ on a logarithmic scale; the blue dotted line is the peak reflectivity in the focal spot. As expected, highest reflectivity occurs for modification factors $f \sim 1$. Also, the phase flatness is enhanced by an order of magnitude or so. The fitted focus sizes are slightly above the diffraction limit (dotted constant line), given by the gradient formula (4.2).

\section{Discussion}

What is the focus size? To quantify the intensity distribution in the focal plane, three different definitions have been used; unfortunately, none seemed to give a "reasonable" 


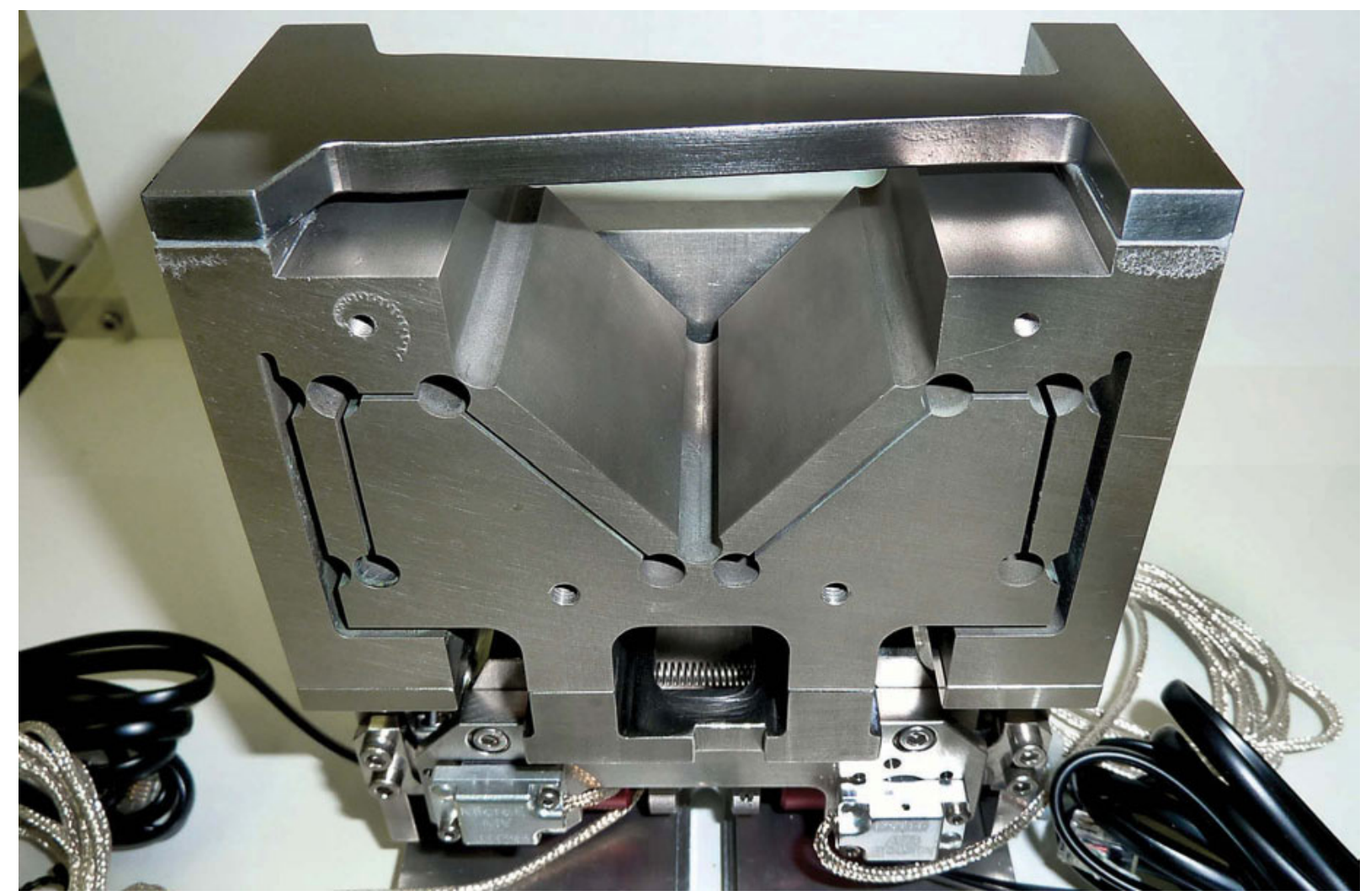

Figure 4.15: Photography of one of the two KB multilayer mirrors on top of a bender currently in use at the ID22NI beamline. Image courtesy: ESRF, Grenoble.

answer. Different definitions of a "focus size" exist, like the Rayleigh criterion (distance between first minima), the full (half) width at half maximum, the root mean square (the $1 \sigma$-value of a Gaussian), and more. For simplicity, let us take the full width at half maximum.

A simple search for fifty percent of the maximum has not proved satisfactory. For experimental data, usually a model function is fitted, and the "focus size" determined from the fit parameters. Here a Gaussian fit (it showed to be very poor) and a $\operatorname{sinc}^{2}$-fit to the propagated intensity have been used. The latter is the model function in the thin lens-approximation. But several fitting procedures each gave inconsistent results when started with different initial values.

In figure 4.17, the fitted "focus sizes", obtained as FWHM values for the sinc $^{2}$-fit, are shown as a function of modification factor $f-$ for different initial values as indicated. Clearly, the nature of the red line (initial value of $\left.1.0 \times \Delta_{0}\right)$ and the blue dotted line $\left(0.8 \times \Delta_{0}\right)$ are totally different. The many plateaus and jumps are hardly satisfactory. In lack of a more adequate mechanism to "measure" the focus, the $\operatorname{sinc}^{2}$-fit was used, with initialisation parameters of $0.94 \times \Delta_{0}$ in figure 4.16 (a) and $0.98 \times \Delta_{0}$ in (b); in figure 4.19 (a), an initialisation of $1.16 \times \Delta_{0}$ and $1.14 \times \Delta_{0}$ has been chosen in (b). These initialisations lead 


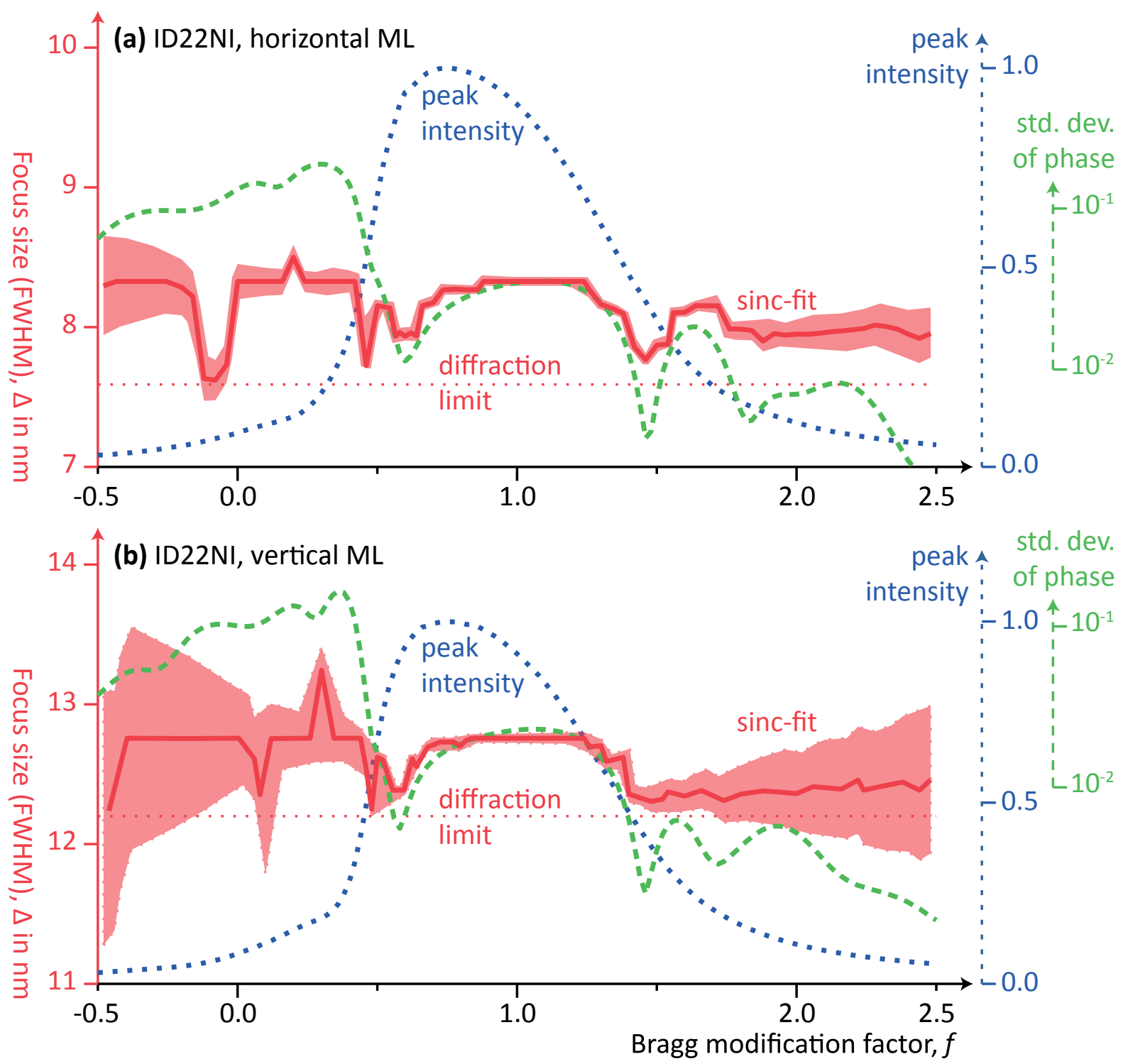

Figure 4.16: Simulated focus sizes for the ID22NI geometry, (a) horizontal ML and (b) vertical ML, as a function of Bragg modification factor $f$. The focus sizes (red curve with reddish error bands) have been obtained from $\operatorname{sinc}^{2}$-fits to the intensity in the focus. Standard deviation of reflected phase (along the ML surface) is shown in green on a logarithmic scale; peak intensity in the focus is shown in blue. Dotted line shows the diffraction limit obtained from the gradient formula, (4.2). Simulation is carried out for a point-source.

to focus sizes consistent with the reflected phase front flatness

$$
\left(\left\langle\phi(s)^{2}\right\rangle-\langle\phi(s)\rangle^{2}\right)^{1 / 2},
$$

where $\phi(s)$ denotes reflected phase along the surface $s$. 


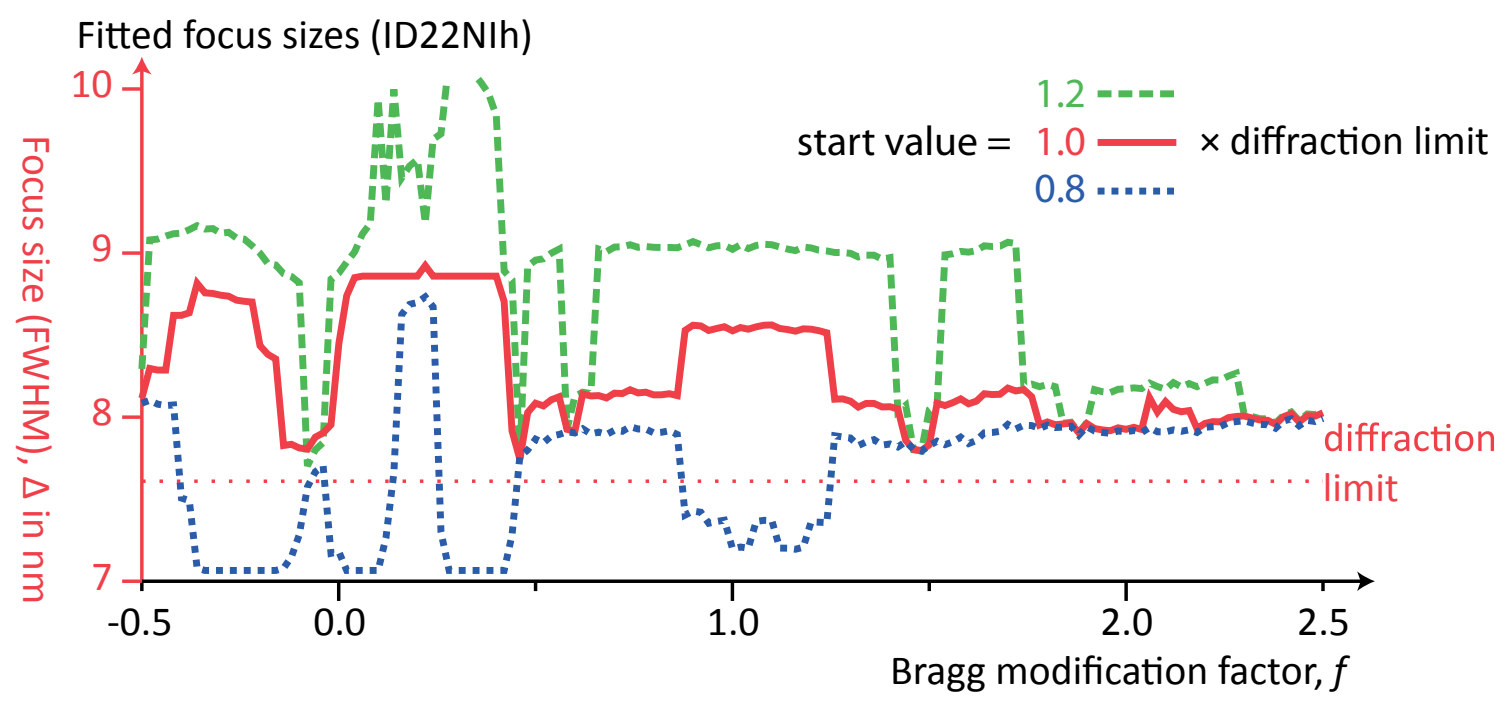

Figure 4.17: Focus sizes as in figure 4.16 (a), but obtained for different start parameters for the fitting procedure. Depending on initialisation, non-linear fitting may produce plateaus and jumps.

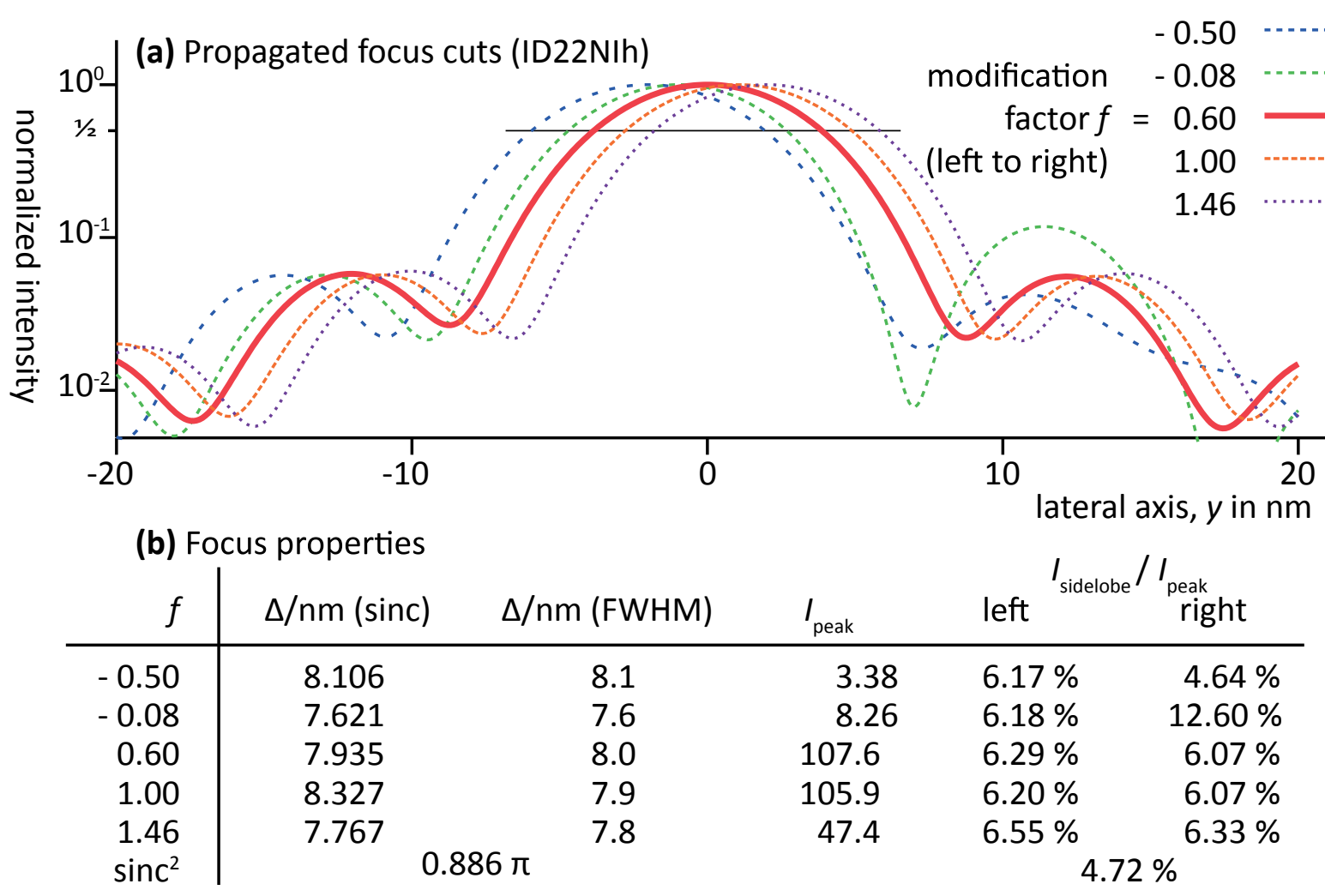

Figure 4.18: Focus cuts for the horizontal set-up in figure 4.16 (a), for different modification factors $f$. Plots in (a) are shifted horizontally and normalised for clarity. In (b), important characteristics are tabulated. Focus size $\Delta$ has been obtained from a $\operatorname{sinc}^{2}$-fit and by searching the $50 \%$-criterion (FWHM). Relative strength of the first side-lobes is given and compared to a perfect $\operatorname{sinc}^{2}$, too. 


\subsubsection{New UPBL04 (NINA-project)}

Analogue simulations as in the previous subsection have been carried out for the aforementioned planned ML KB system to be used in a planned beamline of the ESRF upgrade programme, the UPBL04. This beamline is part of the NINA project" [19].

From the simulations, focus sizes below $6 \mathrm{~nm}$ might be expected. But here it is important to note that the simulations assume a point-source. In horizontal direction, ESRF undulator sources are rather broad and spot sizes below $50 \mathrm{~nm}$ can only be reached with tremendous de-magnification (the ratio $S_{2} / S_{1}$ of distances to the focus / from the source). Additionally, at the ID22NI beamline and the planned UPBL04 it is possible to reduce the horizontal source size by closing slit gaps; however, this reduces flux. Depending on the "virtual source size", nearly diffraction limited focusing is envisioned.

\subsubsection{Exploring limits}

In this subsection we present simulations of multilayer mirrors that touch the physical limits of deposition. The simulations have been performed for four different photon energies, $12.4 \mathrm{keV}, 17.0 \mathrm{keV}, 24.8 \mathrm{keV}$, and $49.6 \mathrm{keV}$. The angles of incidence at the mirrors' centres are varied between $14 \mathrm{mrad}$ and $24 \mathrm{mrad}$, resulting in Bragg bilayer spacings of $0.5 \mathrm{~nm}$ to $3.6 \mathrm{~nm}$. This covers a good part of the range used in current ML mirror depositions, and even going to slightly smaller spacings. The mirrors have a length of $1 \mathrm{~mm}$ or $10 \mathrm{~mm}$; the number of layers varies between 50 and 500 . See table 4.4 for a brief summary of the parameters used throughout this subsection.

The simulated set-ups are defined by the photon energy or wavelength, $\lambda$, and the angle of incidence at the mirror's centre, $\theta$; from that, the angles at the "left" and "right" side of the mirror are derived, depending on mirror length $L$. The results are shown as a function of Bragg-layer spacing $\Lambda=\lambda / 2 \sin \theta$ at the mirror's centre, although simulated using the modified layer spacing (4.18). The set-up and its defining quantities are illustrated in figure 4.20 (a). In part (b), the angular range of a $L=10 \mathrm{~mm}$ mirror is shown as a function of layer spacing and photon energy. A typical pseudo-rocking curve (while an ordinary rocking curve is a function of angle $\Delta \theta$, here the modification factor $f$ is scanned, scaling the modification to Bragg's law) is shown in figure 4.20 (c). The blue line shows the integrated reflectivity of the ML mirror, whilst the green dashed line shows the standard deviation of the reflected phase, on a logarithmic scale. Four points are marked: Bragg's law at $f=0$, the modified Bragg condition at $f=1$, and the points of highest reflectivity and flattest phase; these two points differ in general.

\footnotetext{
"Nanolmaging and NanoAnalysis
} 

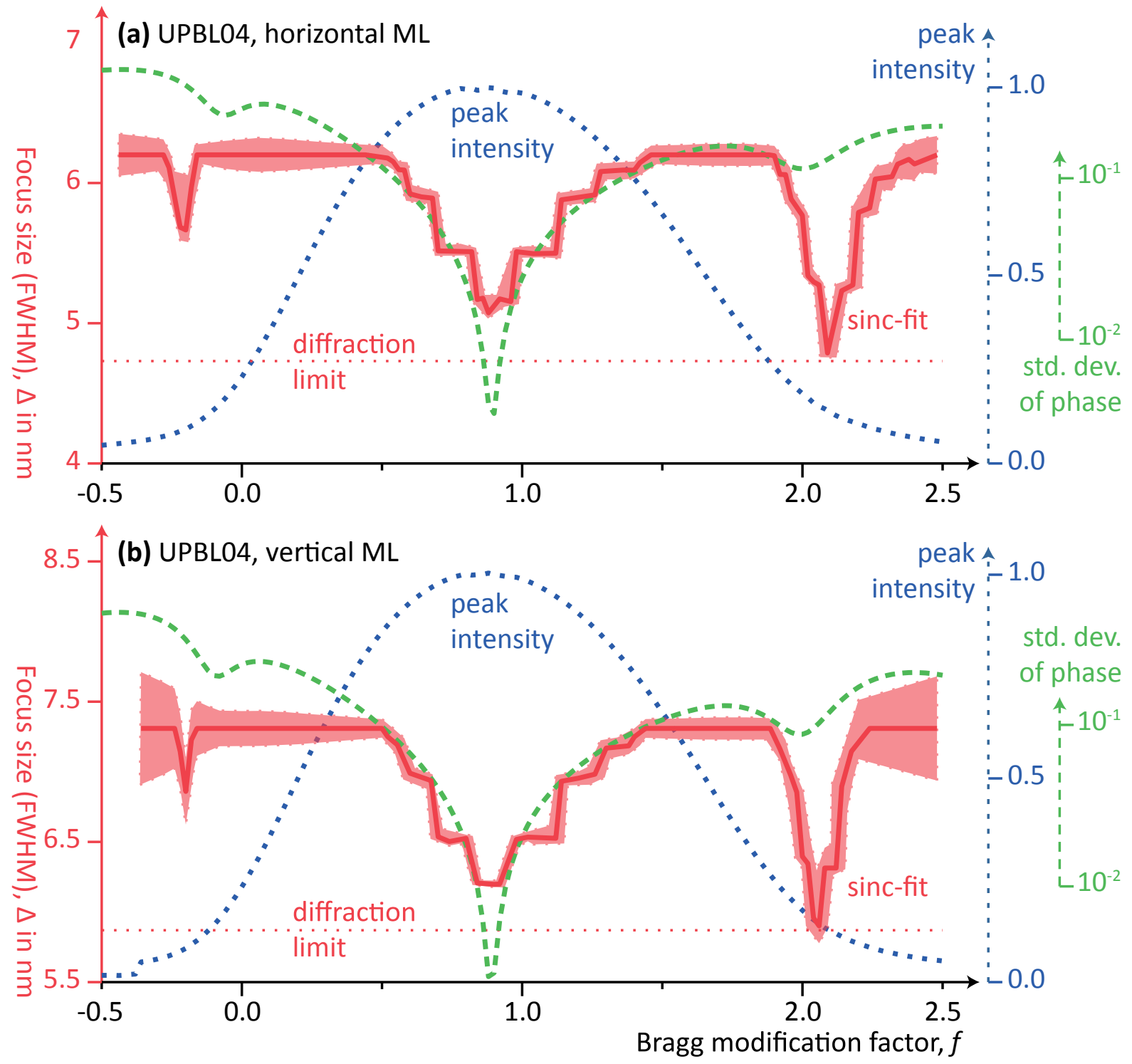

Figure 4.19: Same as figure 4.16, but for parameters of a planned ML KB set-up to be used at the upgrade beamline UPBL04. The geometrical parameters can be found in table 4.3. Simulations have been carried out assuming illumination by a point-source. 


\begin{aligned} Quantity & Value & Quantity & Value \\ \hline distance from source & $145 \mathrm{~m} &$ angle at mirror's centre & $14 \ldots 24 \mathrm{mrad} \\$ distance to focus & $10 \mathrm{~mm} &$ photon energy & $12.4 \ldots 49.8 \mathrm{keV} \\$ length of the mirror & $1 \ldots 10 \mathrm{~mm} &$ number of bilayers & $50 \ldots 500\end{aligned}$

Table 4.4: Geometrical parameters of the model system ML-500.

As already mentioned, the flattest phase in the $f$-scan does not occur for $f=1$. To study the "best" cases, first an optimisation of flattest phase has been carried out for different parameters, the modification factor $f$ was scanned to find the minimum. The result is presented in figure 4.21 (a): The points indicate the modification factor $f(\lambda, \theta)$ as a function of layer spacing $\Lambda=\lambda / 2 \sin \theta$, for the four energies and different angles. Open symbols are simulations for the short $(L=1 \mathrm{~mm})$ mirror, filled symbols those for the long $(L=10 \mathrm{~mm})$ mirror.

Increasing energy and angle of incidence (viz., going to smaller layer spacings $\Lambda$ ), the position of minimal phase-curvature moves towards $f \rightarrow 1$. According to the results, for layer spacings $\Lambda \lesssim 1.5 \mathrm{~nm}$, the modified Bragg condition $f=1$ is a reasonable approximation. At larger spacings, however, less additional material should be deposited. Also, the amount of additional deposition should be decreased for higher layer numbers. The simulations for a photon energy of $17.0 \mathrm{keV}$ (blue lines and symbols) have an overlap with those for $12.4 \mathrm{keV}$ and $24.8 \mathrm{keV}$; obviously, the optimised modified factor depends rather weakly on the photon energy.

The minimum standard deviation of the reflected phases after optimisation is shown in figure 4.21 (b). It is apparent that the shorter mirror yields a flatter phase with $\Delta \phi \lesssim 10^{-4}$, while for the longer mirror only $\Delta \phi \gtrsim 10^{-3}$ can be reached. The flatness improves with photon energy and angle of incidence (viz., towards smaller $\Lambda$ ). In the overlap region, the minimum phase curvature does not depend on photon energy, but only on layer spacing. In the case of the shorter mirror (the lower data points), several outliers are present; it seems as if the phase becomes more strongly curved for higher layer numbers or smaller angles of incidence.

In figure 4.21 (c), the integrated reflectivity along the mirrors' surfaces is shown. While the flatness of the phase does not depend on photon energy, the reflectivity does. This is due to stronger absorption at lower energies. The data shown here are consistent with predictions for the flat ML theory.

Reflectivity curves along the mirrors' surfaces for the points of figure 4.21 , indicated by arrows, are shown in figure 4.22 .

The aforementioned results only considered the reflected field at the mirror's surface. 
Using the mirror simulation programme, described in chapter 3, the field envelope has been propagated to the focal plane, and the focus size has been determined by a sinc ${ }^{2}$-fit. For a discussion of the quality of such fits, see figure 4.17 and the accompanying text.

The simulated focus sizes are shown in figure 4.23. In part (a), the results both for the short mirror of length $L=1 \mathrm{~mm}$ (upper red curve) and for the long mirror, $L=10 \mathrm{~mm}$ (lower blue curve) agree well with the expected diffraction limit using the gradient formula (4.2). The deviations in case of the long mirror become visible in part (b), where the spot size broadening $\Delta-\Delta_{0}$ has been enhanced by a factor of 20; see the "broadening axis" shown on the right. The deviations are on the order of $0.1 \mathrm{~nm}$ to $0.25 \mathrm{~nm}$, which corresponds to about five to ten percent. This broadening can be attributed mainly to two causes: (i) at high photon energies the reflectivity is not homogeneous, hence effective aperture is reduced; (ii) the $\operatorname{sinc}^{2}$-model is based on the assumption of a thin lens, which is not the case here.

It should again be emphasised that all focus simulations presented in this chapter assume a point-source, fully coherent in temporal and spatial domain. Calculations incorporating spatially partial coherence as shown for simple mirrors in chapter 3 should be possible, using the scheme from chapter 2.

Using numerical simulations, the field inside a focusing multilayer mirror has been calculated and the exiting wave envelope has been propagated to the focus. The modified Bragg condition yields enhanced reflectivity; influence on the focus size, however, is very weak. 
(a) Defining quantities $L^{*}$

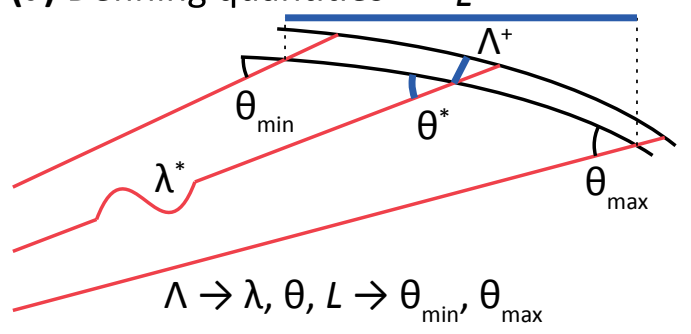

(b) Angular range of different mirrors

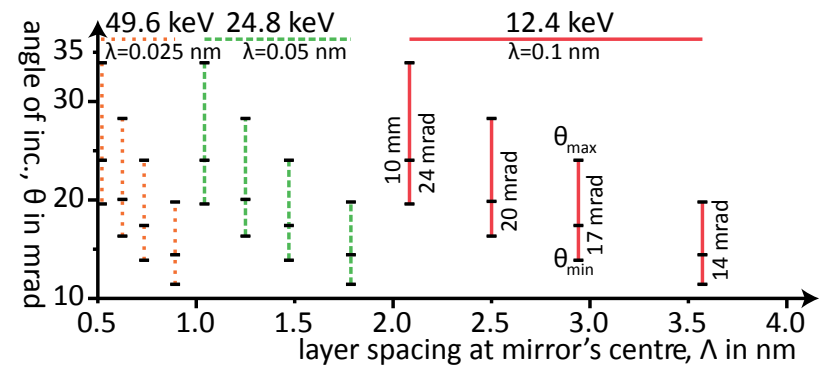

(c) Modified rocking curve

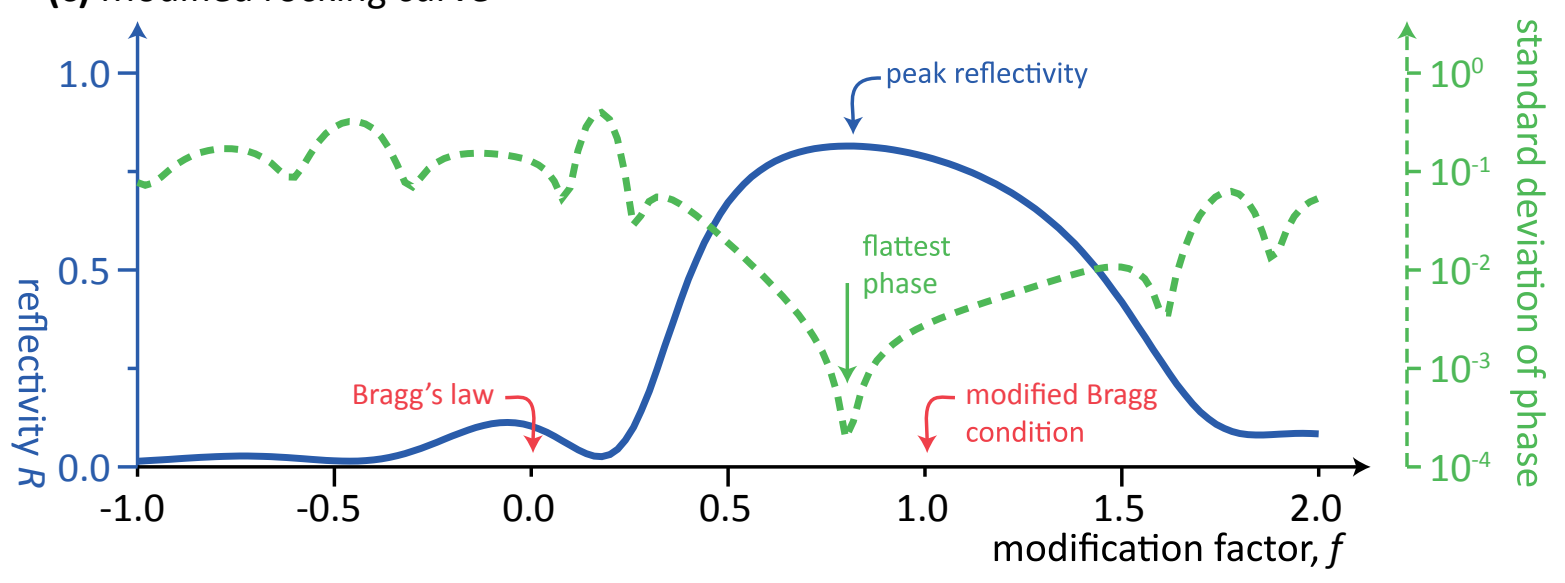

Figure 4.20: Definition of the simulation set-up for the following results. (a) Geometrical definition: for different wavelengths $\lambda$ and mirror lengths $L$, the angle of incidence at the mirror's centre, $\theta_{c}$, is varied; in (b) the resulting minimal and maximal local angles of incidence on the "left" and "right" edge are shown as a function of layer spacing at the mirror's centre. Scanning the Bragg modification factor $f$, a pseudo-rocking curve as shown in (c) can be simulated. The blue line is the integrated reflectivity of the mirror, while in green the standard deviation of the reflected phase (an estimation of the flatness of the phase) is shown on a logarithmic scale. Quantities in (a) marked by an asterisk define the geometry, while those marked with a plus sign is the abscissa of the following plots. 

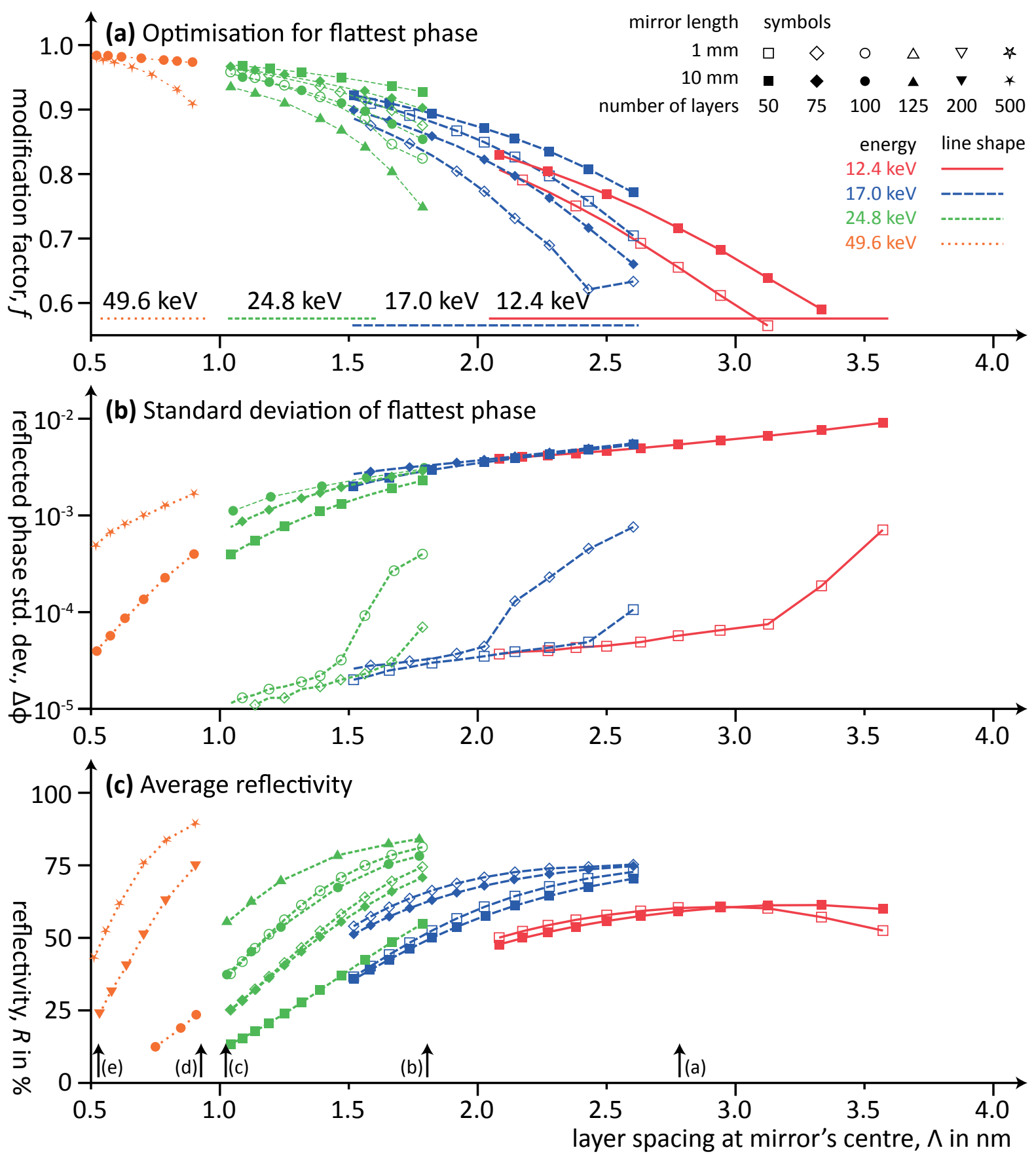

Figure 4.21: Optimisation of $M L$ mirrors for the considered geometry. In (a), the optimised modification factor $f$ as defined in (4.18) is shown as a function of Bragg layer spacing at the mirror's centre, while (b) shows the phase-flatness for the optimised $f$-values. In (c), the average reflectivity of the optimised ML mirrors is shown. Different line shapes and colours represent energy, symbols denote mirror length and layer number; the angle of incidence varies from $14 \mathrm{mrad}$ on the right to 24 mrad on the left of each curve. 


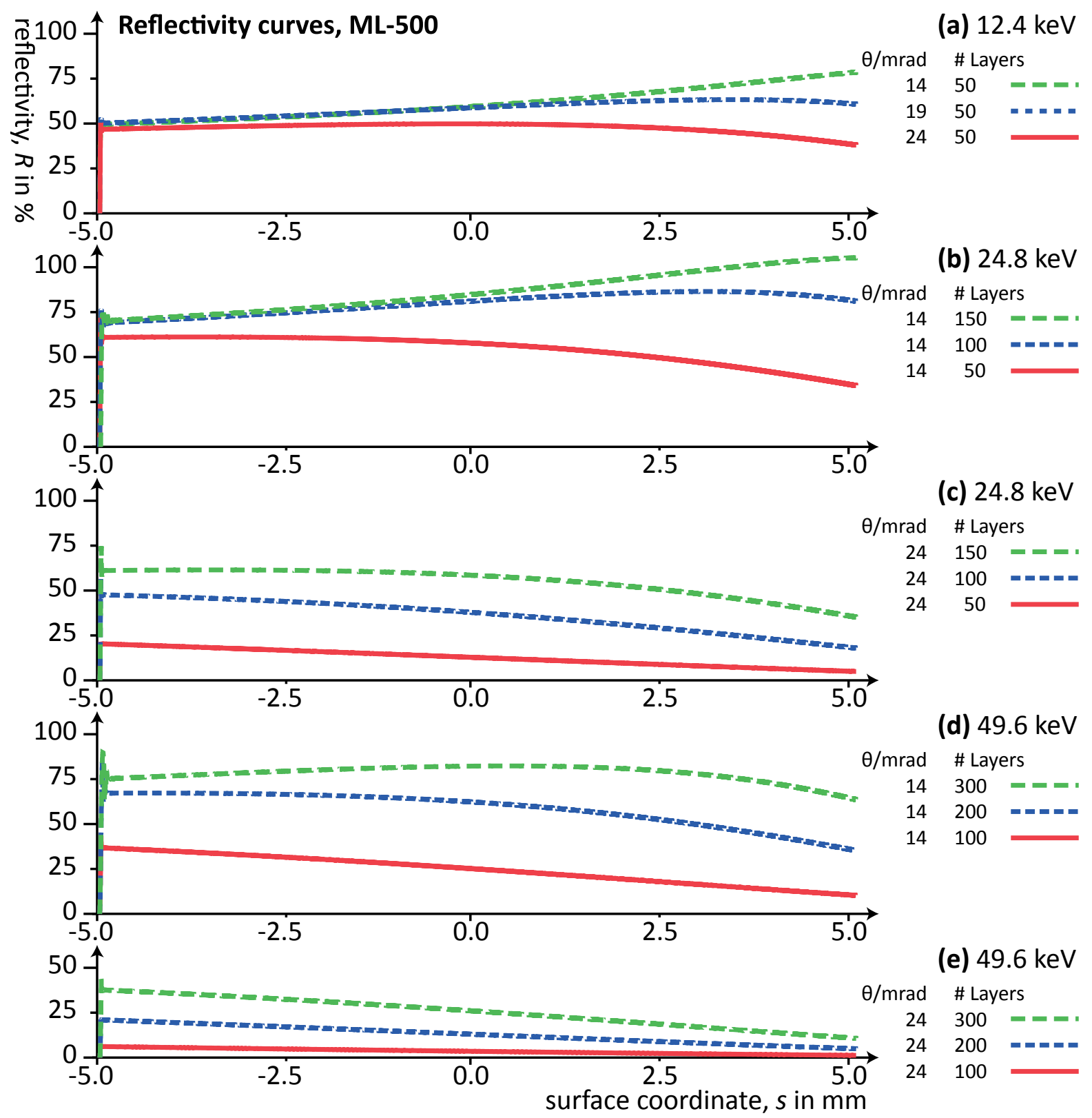

Figure 4.22: Local reflectivity curves along the multilayer surface, at the points indicated in figure 4.21. For a discussion see the text. 
(a) Simulated focus sizes

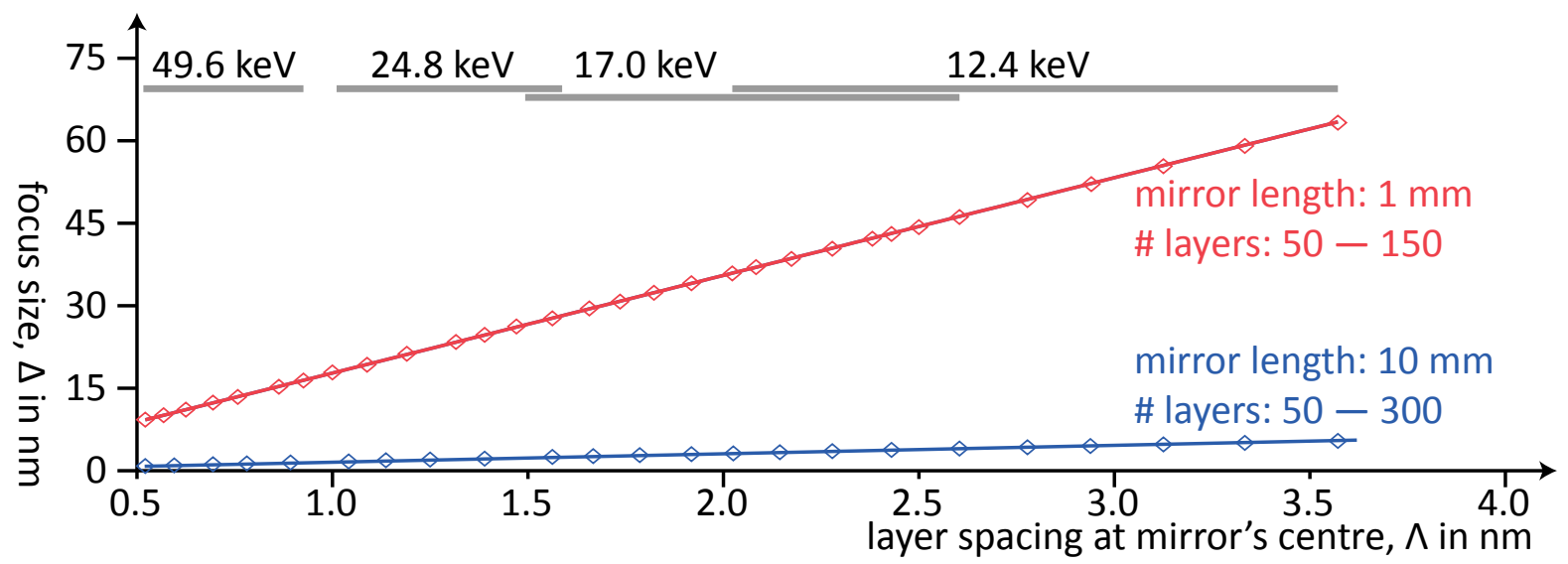

(b) Deviations from diffraction limit, mirror length: $10 \mathrm{~mm}$

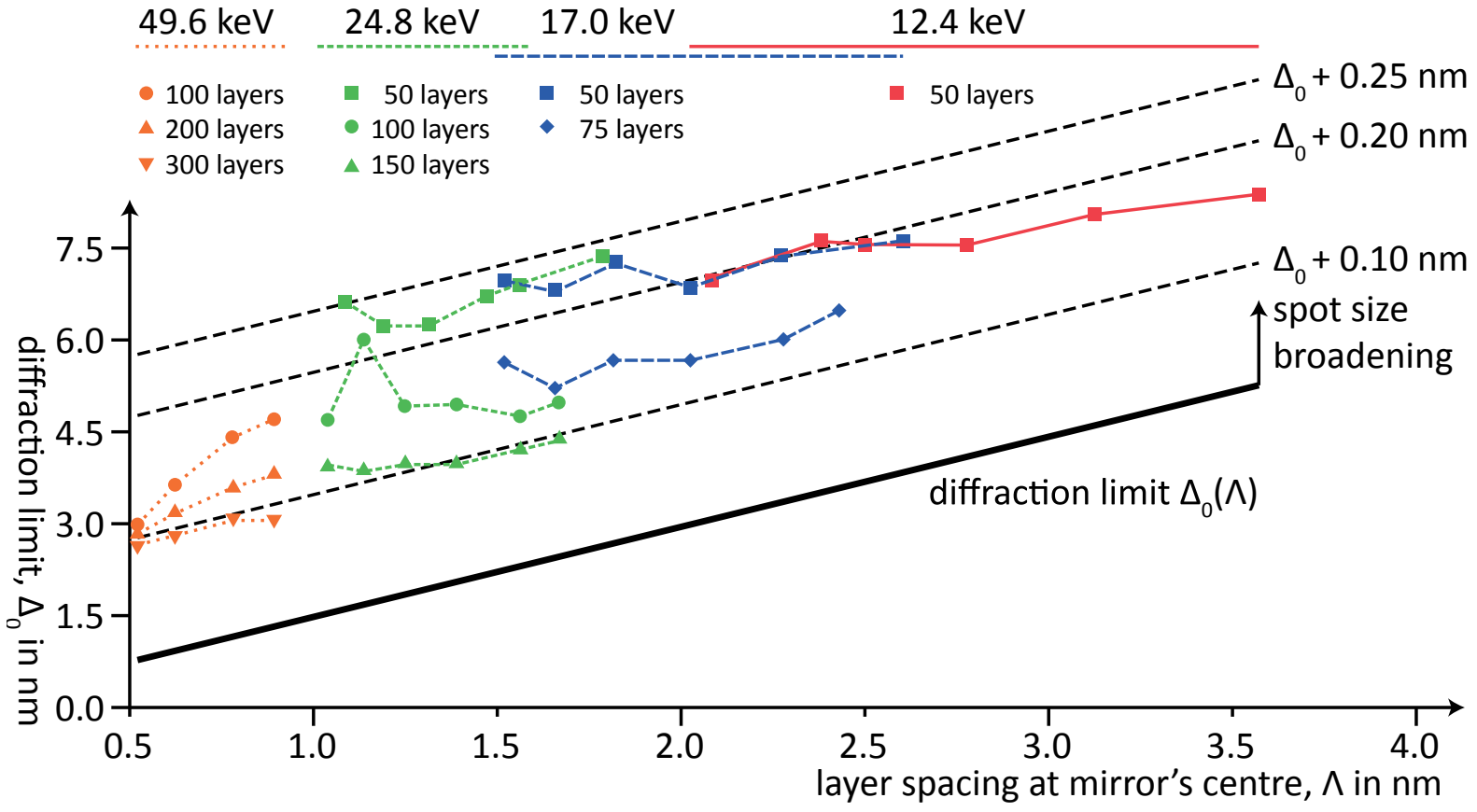

Figure 4.23: Simulated focus sizes for the ML-500 set-up, optimised for "flattest phase". In (a), the simulated focus sizes for the short mirror (red curve, $L=1 \mathrm{~mm}$ ) and the longer mirror (blue curve, $L=$ $10 \mathrm{~mm}$ ) are shown, together with lines representing the diffraction limit. In (b), the deviations from diffraction limit for the long mirror is shown. The abscissa measures the diffraction limit, while the symbols are placed according to the "spot size broadening" axis shown on the right. As can be seen, the simulated focus sizes are within $0.1 \ldots 0.25 \mathrm{~nm}$ of the diffraction limit, as predicted by the gradient formula (4.2). For details see the text. 


\section{Chapter 5}

\section{Waveguides}

\subsection{Introduction}

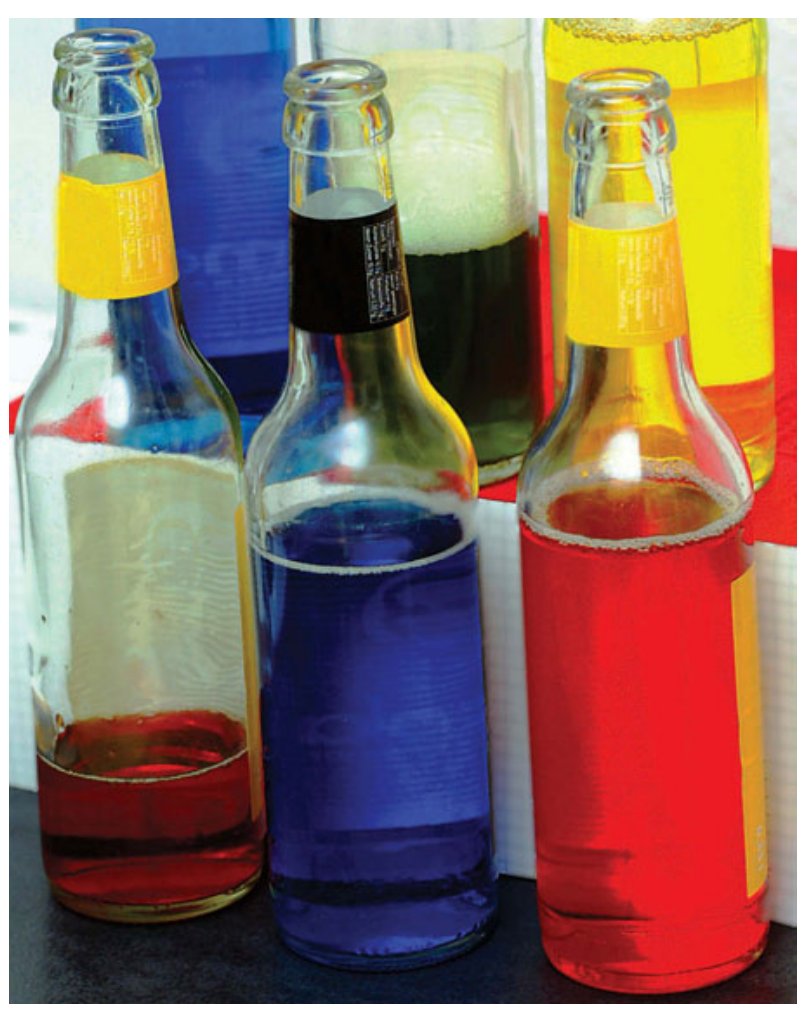

In chapter 2 both an analytical and numerical treatment for coherence propagation in x-ray waveguides has been proposed. In this chapter we present results using both approaches. Analytical results often provide qualitative insights for questions with easy geometries and using approximations. Numerical results, on the other hand, can be used more flexibly also for complex geometries and for more complicated expressions. However, numerical inaccuracies obscuring the results have to be taken into account. While setting up analytical expressions for the degree of coherence in $\mathrm{x}$-ray waveguides we only considered guided modes, neglecting radiative ones. In a numerical treatment these are accounted for. Nevertheless the analytical expressions provide a quick and comprehensive answer, for example when optimising waveguide length in terms of coherence filtering (see subsection 5.2.4).

Using the analytical framework from subsection 2.2.6, we will calculate the evolution of coherence properties inside $\mathrm{x}$-ray waveguides. We start with a toy model that shows important features of $|j|$. Solving an overlap integral of discrete modes with a model illumination function, semi-analytical results follow next. In subsection 5.2.4, the required waveguide length for a specified degree of coherence (between points on optical axis and channel boundary) is evaluated.

Results based on numerical propagation are presented in section 5.3. These calculations include effects due to radiative modes which add incoherently to the guided modes and are neglected in the analytical approximation of section 5.2. We close with section 5.4 about combined optics, that is waveguides placed in the focus of an $\mathrm{x}$-ray focusing mirror. X-ray waveguides are usually put into a pre-focused synchrotron beam, both to enhance coherence 


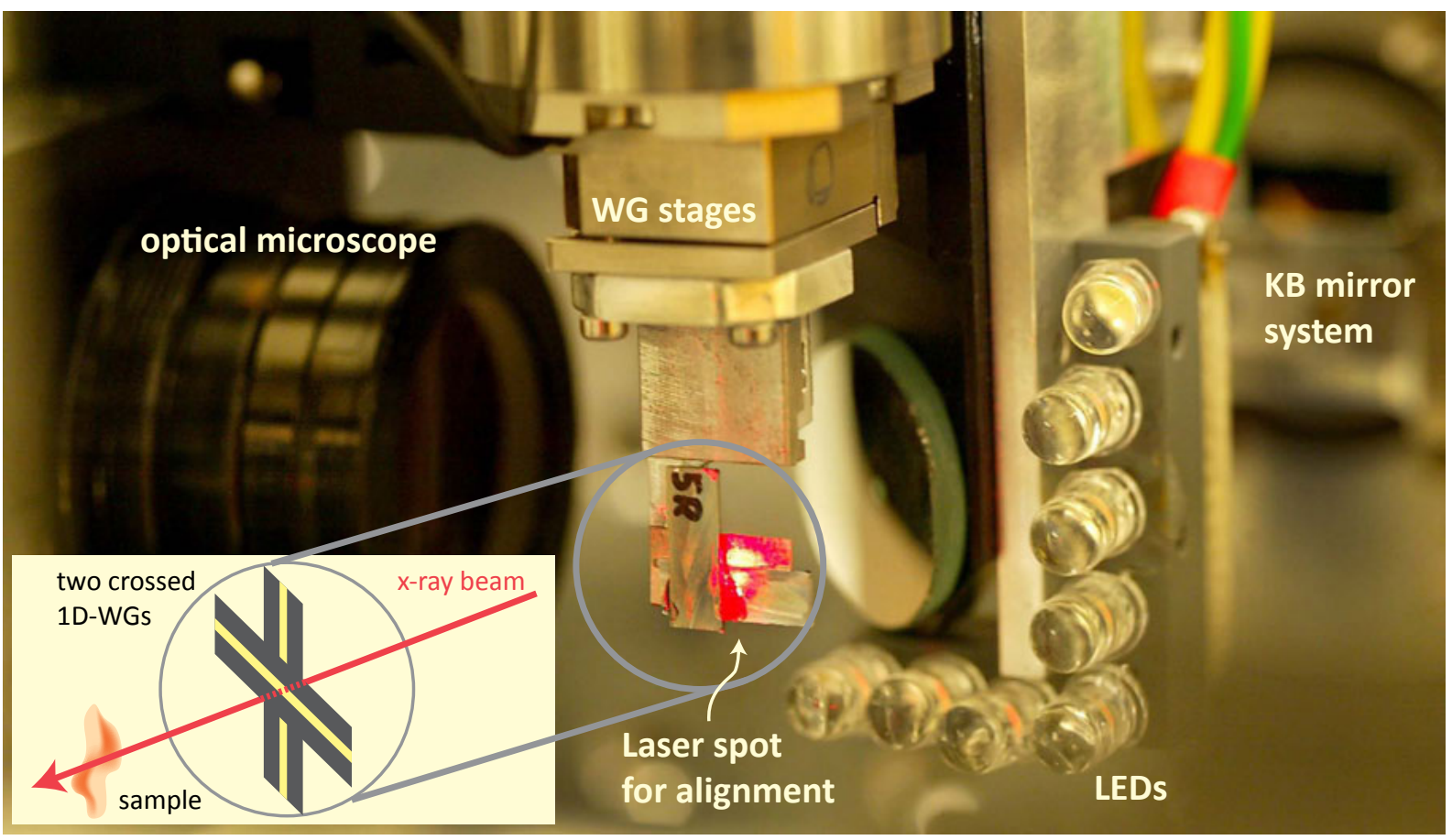

Figure 5.1: Photo of an experimental set-up for waveguide based propagation imaging at the coherence beamline P10 at PETRA III. As illustrated in the inset, two planar waveguides in crossed geometry are attached to several stages for rotation and translation. The pre-focused x-ray beam illuminates from the right. LEDs illuminating the sample (not shown) and an optical microscope in the background help in aligning of the set-up.

properties and to clean-up the beam; a photo of an experimental waveguide set-up used for imaging at the coherence beamline P10 at PETRA III is shown in figure 5.1.

The results presented in this chapter have already been published in [61].

\subsection{Analytical degree of coherence}

In subsection 2.2.6, an analytical expression for the degree of coherence $j(x, d)$ in an x-ray waveguide, after a propagation distance $x$, between the optical axis and a point within a lateral distance $d$, was derived to be

$$
j(x, d)=\frac{\sum_{n} \lambda_{n}(x) \psi_{n}^{*}(0) \psi_{n}(d)}{\sqrt{\sum_{n} \lambda_{n}(x)\left|\psi_{n}(0)\right|^{2} \cdot \lambda_{n}(x)\left|\psi_{n}(d)\right|^{2}}} .
$$

The occupation numbers $\lambda_{n}(x)$ decay exponentially due to absorption, since a fraction of the mode's energy propagates inside the cladding material. Their start values $\lambda_{n}(0)$ have to be determined by an overlap integral of the mode with an incoming field (see subsection 
5.2.2). But first, we examine $j(x, d)$ in a toy model for a set of chosen model occupation numbers in the following subsection. Numerically solving the overlap integral, propagation of the analytical degree of coherence is shown in subsection 5.2.3. In the final part of this section we address the question of minimum waveguide length if a specified degree of coherence is needed for an experiment. The following section 5.3 then presents results obtained from numerical propagation.

\subsubsection{Guided modes in a toy model}

The degree of coherence $j(x, d),(5.1)$, primarily depends on the occupation numbers $\lambda_{n}$. As a first step we just "guess" a set of $\lambda_{n}$ to get first insights of the behaviour of $j$. However, in the following examples we have the set-up of a silicon-vacuum-silicon waveguide with a guiding layer thickness of $D=70 \mathrm{~nm}$ in mind, hence up to four modes are guided.

We fix $\lambda_{1}=1.0$ and then add, step by step, the higher modes. Figure 5.2 shows $j(d)$ for two (a), three (b), and all four (c) modes excited. In each part, the highest mode's strength is increased from red over blue to green. The actual $\lambda_{n}$ are included in the key. The boundary between guiding layer and cladding material at $d=35 \mathrm{~nm}$ is indicated by the dotted line. Exciting only two modes, the degree of coherence decreases with increasing $\lambda_{2}$. In (b), the third mode is switched on, and a root of $|j|$ appears; actually, this is a zero-crossing of $j$ : the modes are anti-correlated* Turning on the fourth mode, as in (c), the finite anti-correlation for large separations vanishes.

\subsubsection{Overlap integral}

Next we calculate the occupation numbers for given waveguides geometries and illumination function. Since the degree of coherence $j$ only depends on the ratios of the $\lambda_{n}$, we can agree to use relative occupation numbers that add up to 1.0. As discussed in the literature $[31,33,102]$, the occupation numbers are given by the overlap integral of the respective modes $\psi_{n}(y)$ with the illumination function $\psi_{\text {illu }}(y)$.

Waveguides used for coherence filtering are of course illuminated by a partially coherent beam. We assume that $\psi_{\text {illu }}(y)$ is composed of independent, hence incoherent, plane waves

${ }^{*}$ Anti-correlated fields yield an interference pattern in the far-field; but compared to the case of correlated fields, minima and maxima are swapped. 


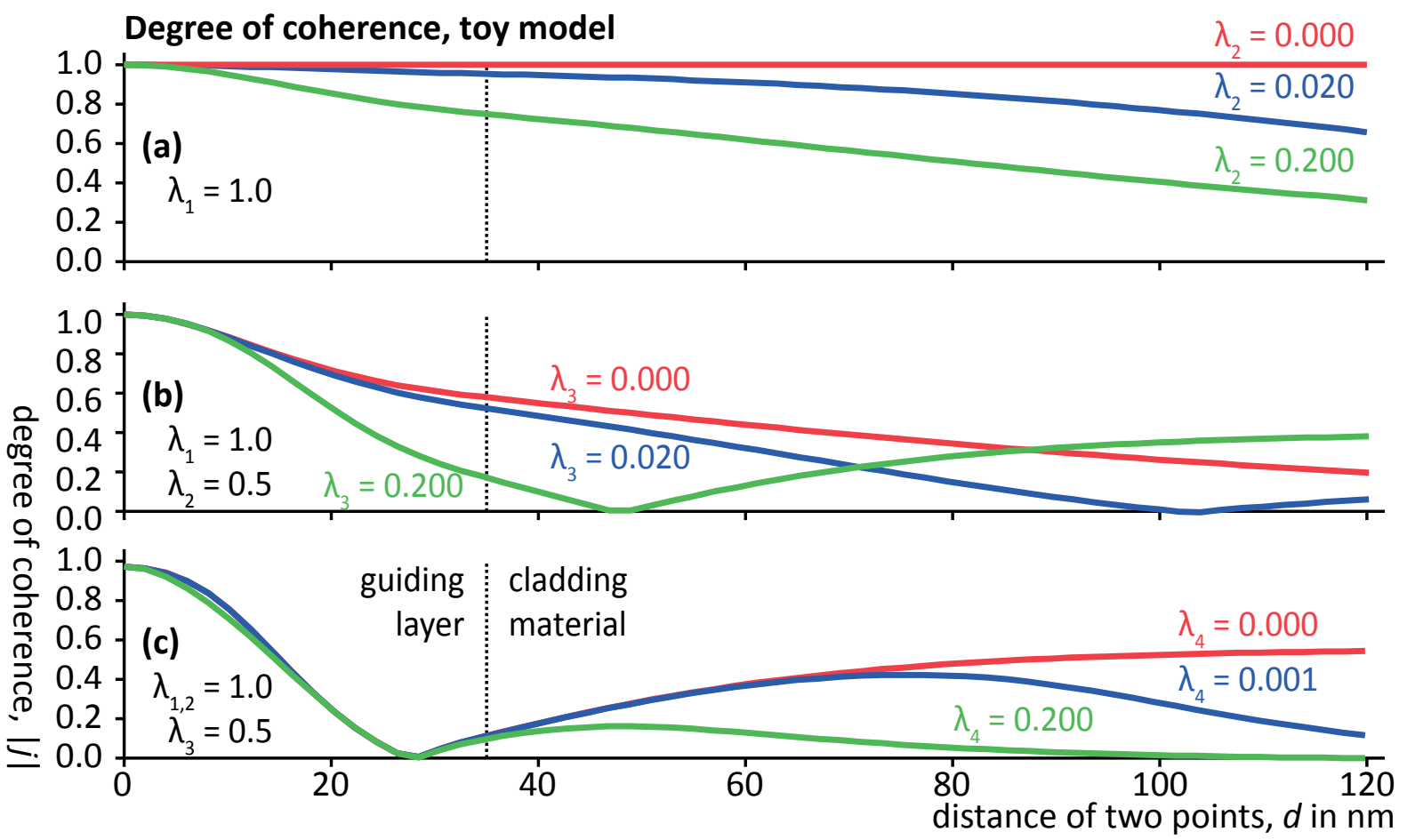

Figure 5.2: Analytical degree of coherence in a toy model $x$-ray waveguide supporting up to four modes. From (a) to (c), two, three, and four modes are excited, with increasing occupation numbers as given by the $\lambda_{n}$. The dotted line shows the interface between guiding layer and cladding; a significant fraction of intensity is located within the guiding layer.

$\psi_{\text {illu }}(y, \theta)$ impinging under an angle $\theta$ perpendicular to the entrance,

$$
\begin{aligned}
\psi_{\text {illu }}(y, \theta) & =\psi_{\text {env }}(y, \theta) \exp \left(\frac{2 \pi i}{\lambda} \sin \theta\right) \\
\psi_{\text {env }}(y, \theta) & =\psi_{\text {env }} \exp \left(-y^{2} / 2 \sigma_{y}^{2}\right) \exp \left(-\theta^{2} / 2 \sigma_{\theta}^{2}\right) .
\end{aligned}
$$

(Here, $\lambda$ is the wavelength.) The incoming angular spectrum and each plane wave is thus enveloped by Gaussians of widths $\sigma_{\theta}$ and $\sigma_{y}$. Each plane wave shall excite the modes independently, we write the overlap integral for angle $\theta$ as

$$
\lambda_{n}(\theta)=\left|\int_{-\infty}^{\infty} \mathrm{d} y \psi_{\text {illu }}(y, \theta) \cdot \psi_{n}(y, \theta)\right|^{2} .
$$

To calculate the total occupation numbers $\lambda_{n}$, we integrate over angles:

$$
\lambda_{n}=\int \mathrm{d} \theta \lambda_{n}(\theta)
$$

For finite integral limits and a more realistic model, we limit the illumination envelopes by setting $\sigma_{y}=5 D$ and $\sigma_{\theta}=5 \mathrm{mrad}$ ( $D$ is the guiding layer thickness). The former 
yields a finite illumination beam, while the latter restricts the angular spectrum. The small value of $5 \mathrm{mrad}$ is reasonable since the angular acceptance of $\mathrm{x}$-ray WGs is limited by the critical angle $\vartheta_{c}$. For silicon at photon energies of $E=12.4 \mathrm{keV}$, we have $\vartheta_{c}=2.52 \mathrm{mrad}$. The lateral beam size $\sigma_{y}=5 D$ corresponds to about $100 \mathrm{~nm}$ to $1 \mu \mathrm{m}$ (full width at half maximum, FWHM), which is a reasonable model for good or moderate pre-focusing available at synchrotron radiation sources.

The integrations are carried out numerically by Riemann sums. As integration limits, $\pm 5 \sigma_{y}$ and $\pm 2 \sigma_{\theta}$ have been chosen; the integration domain was divided into $1000 \times 500$ (in $y$ and $\theta)$ points. Solving the overlap integral, occupation numbers $\lambda_{n}(x=0)$ are obtained.

The modes are subject to absorption, since a finite fraction of energy is transported inside the cladding material. If the index of refraction is written as $n=1-\delta+i \beta$, the linear absorption coefficient for Beer-Lambert's law is $\mu=2 k \beta$ [8], and the propagated occupation numbers $\lambda_{n}(x)$ are

$$
\lambda_{n}(x)=\lambda_{n} e^{-\mu_{n} x}
$$

Here the effective absorption per mode is given by the fraction of intensity inside the cladding,

$$
\mu_{n}=\mu_{\mathrm{cl}}(E)\left(\int_{y \in \text { cladding }} \mathrm{d} y\left|\psi_{n}(y)\right|^{2}\right) /\left(\int \mathrm{d} y\left|\psi_{n}(y)\right|^{2}\right),
$$

and $\mu_{c l}(E)$ is a tabulated value depending on photon energy and cladding material. In figure 5.3 the relative occupation numbers $\lambda_{1 \ldots 4}$ for WG guiding layer thickness $D=10 \ldots 70 \mathrm{~nm}$ (i.e. one to four modes) are shown (a) right at the entrance, and after (b) $500 \mu \mathrm{m}$, and (c) $1500 \mu \mathrm{m}$ of propagation, for $E=12.4 \mathrm{keV}$ and a silicon cladding. Limiting values for $\sigma_{y}, \sigma_{\theta} \rightarrow \infty$ are indicated by the dotted lines.

Obviously, right at the entrance the occupation numbers resemble their limiting values for $\sigma \rightarrow \infty$ quite well; as soon as a specific mode $n$ is allowed to propagate, relative occupation numbers go to $1 / n$. Due to finite beam size and reduced angular spectrum the steps are smoothed. After some propagation - $500 \mu \mathrm{m}$ in (b) and $1500 \mu \mathrm{m}$ in (c) - the highest modes are absorbed quickly and hence the low modes dominate. From this one expects an increase in degree of coherence due to mode filtering.

The following subsections will give more quantitative testament of this expectation. 

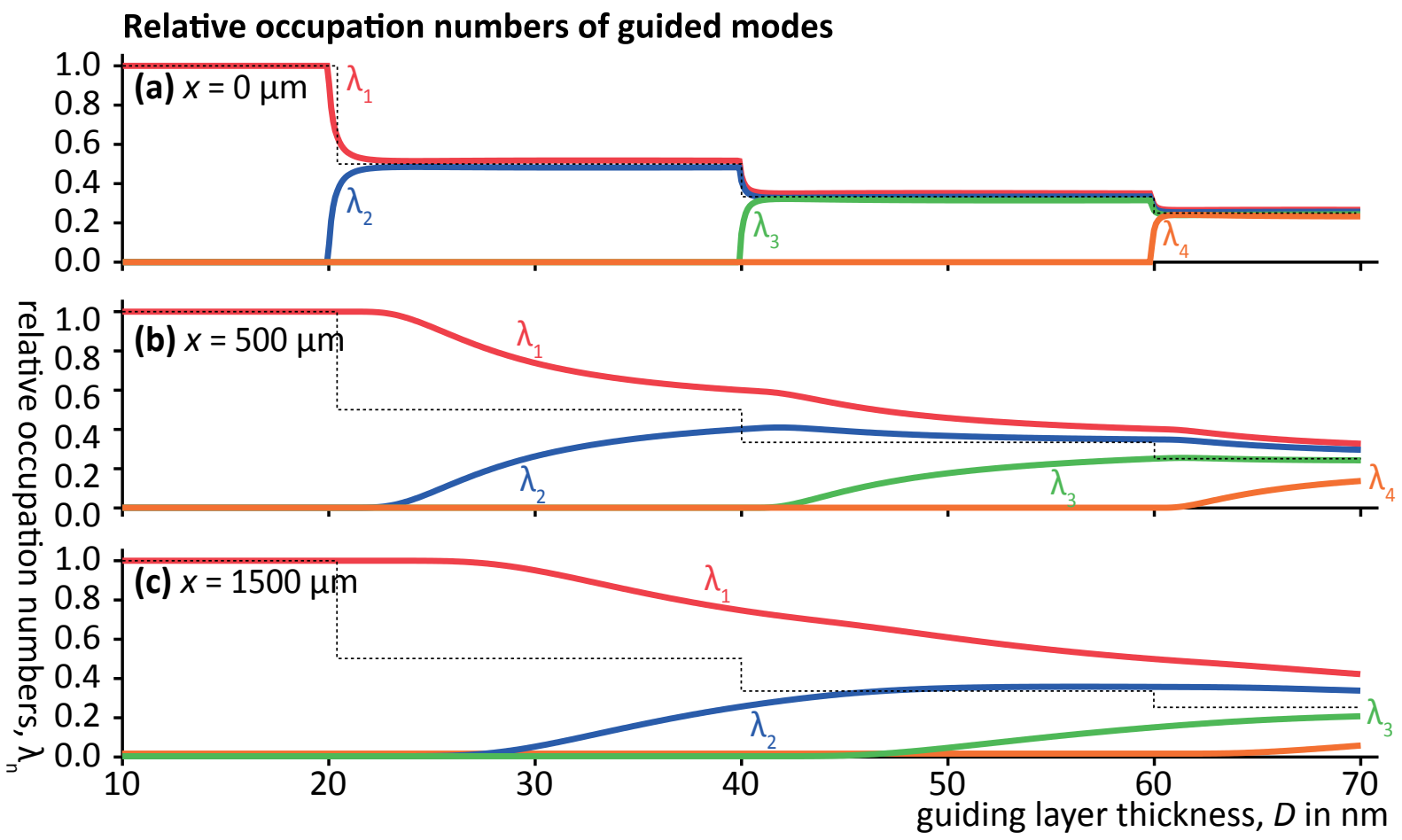

Figure 5.3: Relative occupation numbers $\lambda_{n}$ as a function of WG guiding layer thickness $D$, and for different propagation lengths, (a) to (c). The parameters of the illumination are given in the text.

\subsubsection{Degree of coherence}

Based on the occupation numbers calculated in the previous subsection, we now present the resulting degree of coherence, $j(x, d)$. The coordinate $x$ measures distance from the waveguide's entrance, while $d$ is the distance from the probe point to the optical axis.

Figure 5.4 shows the degree of coherence for three WGs with $D=\{30,50,70\} \mathrm{nm}$ and propagation lengths $x=\{0,500,1500,6000\} \mu \mathrm{m}$. As can be seen, the degree of coherence increases considerably with propagation distance, resulting from the stronger absorption of higher modes, and the resulting smaller spectrum of mode occupation numbers.

Compared to figure 5.2 and our toy model, the guessed occupation numbers describe the actual curves quite well. See, for example, at the green curve in figure 5.4 (a), with guiding layer thickness $D=30 \mathrm{~nm}$ and propagation distance $x=1500 \mu \mathrm{m}$ : since the second mode is absorbed stronger than the first mode, the degree of coherence increases as a function of propagation distance $x$. After a few millimetres, the beam is nearly fully coherent.

In the case of a three-mode-WG, figure 5.4 (b), with $D=50 \mathrm{~nm}$, coherence filtering by mode damping takes longer. First, the anti-correlation far in the cladding is reduced. 
Degree of coherence, guided modes

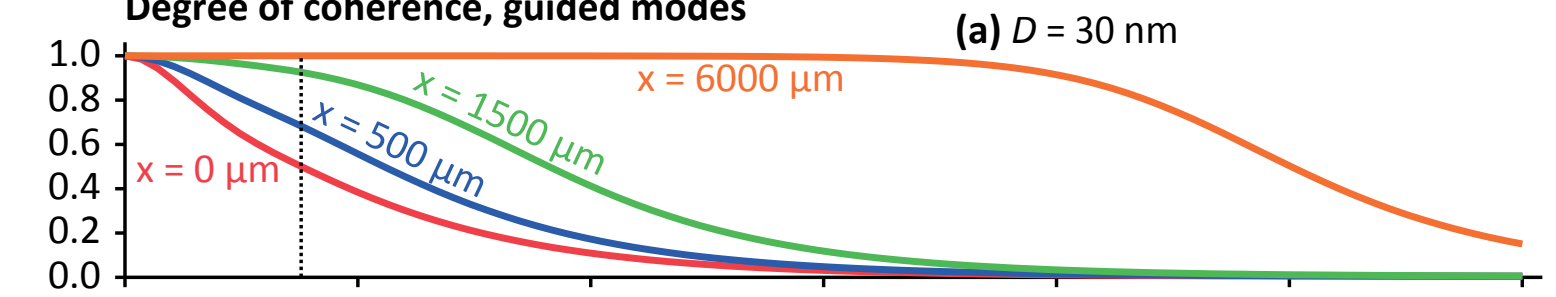

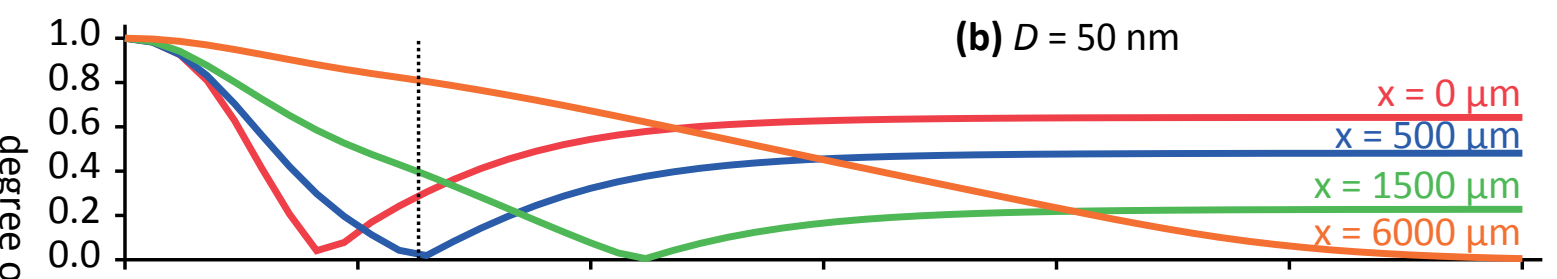

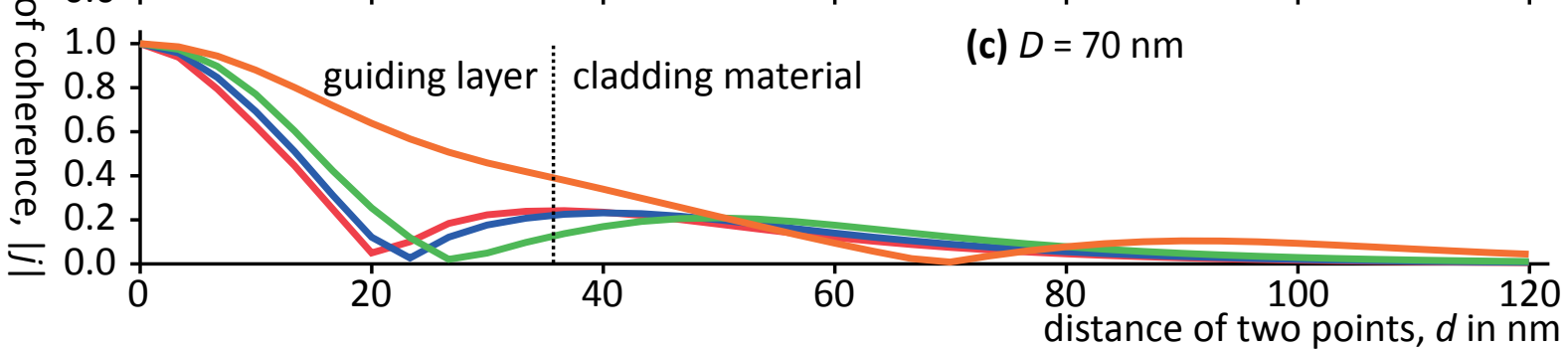

Figure 5.4: Degree of coherence $|j|$ for three waveguides of different guiding layer thickness $D$, (a) to (c), and different propagation lengths $x$.

Near the interface between guiding layer and cladding, a finite correlation at the entrance vanishes for some hundred micrometres of propagation, while the zero-crossing moves to larger separations. After propagation lengths of about $6 \mathrm{~mm}$, the confined beam is highly correlated.

For a four-mode-WG as in figure 5.4 (c), $D=70 \mathrm{~nm}$, propagation lengths of $x=6 \mathrm{~mm}$ are not sufficient to filter photon energies of $E>12.4 \mathrm{keV}$. The required WG length for a specified degree of coherence threshold is the topic of the following subsection.

\subsubsection{Optimised waveguide length}

Due to absorption of higher modes, the degree of coherence is enhanced with increasing propagation distance $x$ in $x$-ray waveguides. In this subsection we address the question of minimum WG length, if a certain degree of coherence $j(x, d=D / 2)$ is required between optical axis and interface. Figure 5.5 gives two answers: in (a) the energy is fixed, and required WG length $L$ is shown as a function of guiding layer thickness for different thresholds, 


\section{Required waveguide length for threshold degree of coherence}

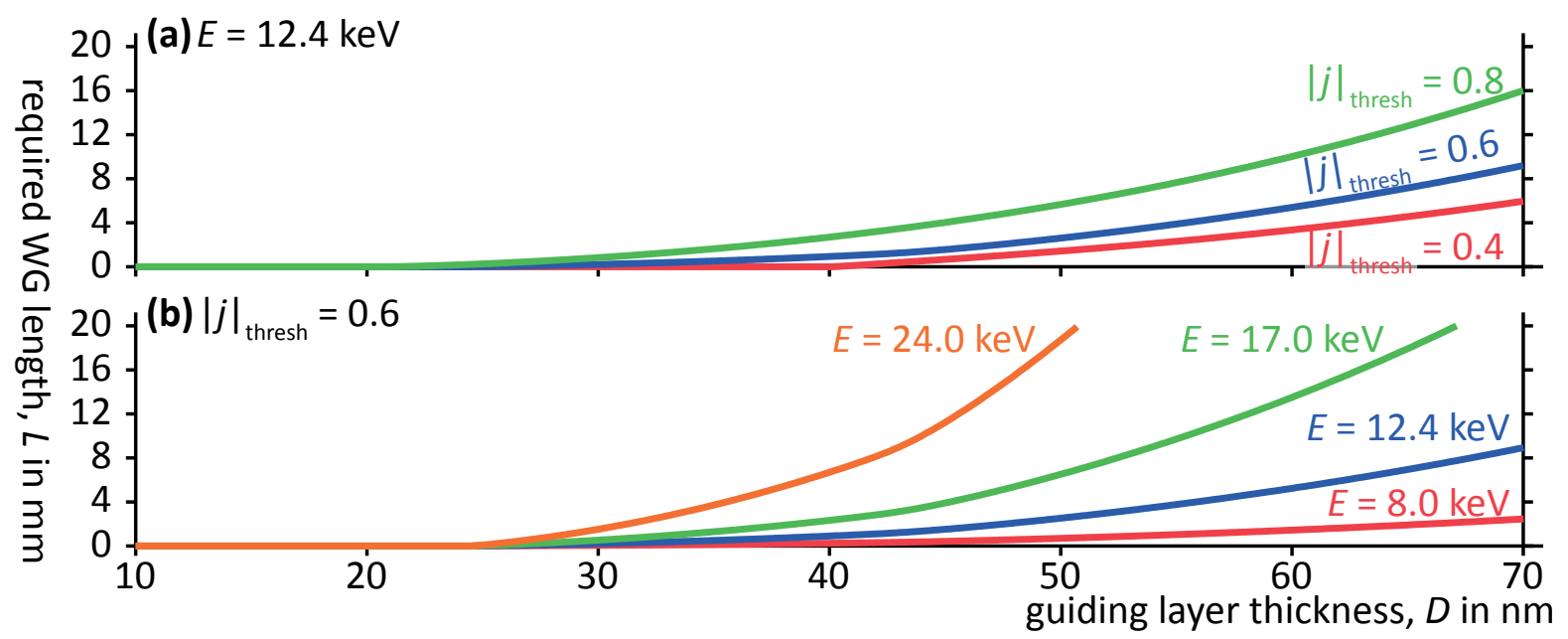

Figure 5.5: Required waveguide length $L$ as a function of guiding layer thickness $D$, for different energies $E$ and coherence thresholds $j_{\text {thresh }}$ between the optical axis and the interface.

i.e.

$$
L\left(D, j_{\text {thresh }}\right)=\operatorname{argmin}_{x}\left(j(x, d=D / 2) \geq j_{\text {thresh }}\right)
$$

for (b), on the other hand, a constant threshold $j_{\text {thresh }}=0.6$ was chosen and the required length calculated for different photon energies, hence

$$
L(D, E)=\operatorname{argmin}_{x}\left(j(x, d=D / 2)_{E} \geq 0.6\right) .
$$

At a fixed energy, $E=12.4 \mathrm{keV}$, required length scales nearly quadratically with guiding layer thickness. This is a result of the modes' extension into the cladding quickly being reduced, and thus the effective absorption $\mu_{n}$. For this moderate energy, the four-mode WGs up to $D=70 \mathrm{~nm}$ can be filtered efficiently with WG lengths in the millimetre range $\left(L \approx 9 \mathrm{~mm}\right.$ for $\left.j_{\text {thresh }}=0.6\right)$. If a higher degree of coherence is necessary, the guiding layer thickness should be reduced to $D \lesssim 55 \mathrm{~nm}$ for comparable lengths.

Figure 5.5 (b) shows the results for a fixed, medium threshold $j_{\text {thresh }}=0.6$, at different energies up to $E=24 \mathrm{keV}$. As can be seen, WGs with $D \gtrsim 50 \mathrm{~nm}$ are not favourable if $E \gtrsim 17 \mathrm{keV}$, because very long WGs would be required for coherence filtering.

It has to be noted that due to radiative modes not being considered here actual degrees of coherence are smaller; this will be shown in the next section by numerical propagation. Coherence properties increase for reduced angular spectra, however. 
Based on analytical theory, the degree of coherence has been propagated through $\mathrm{x}$-ray waveguides. With the described framework it is now possible to optimise WG design in terms of coherence. However, radiative modes and real structure effects are beyond this simple analytical approach. Numerical propagation as described in the following section is able to account for these effects. 


\subsection{Numerically propagated degree of coherence}

In this section, results from numerical propagation including radiative modes are presented. The code is based on the programme PWG (parabolic wave equation GUI) by Christian Fuhse [33] that propagates a single plane wave along an x-ray waveguide and uses the parabolic wave equation, an approximation of the Helmholtz equation. Some improvements have been made:

1. the code was ported from $\mathrm{IDL}^{\dagger}$ to $\mathrm{C}$-code and optimised for speed,

2. free-space propagation before and after the WG is included.

With this new version and the tools for stochastic superpositions (see chapter 2), fields can be propagated and superposed much faster than before; the typical time-scale for Si-WGs with lengths of a few millimetres is less than a minute for each incident wave. In figure 5.6 (a), the intensity distribution inside a Si-WG of guiding layer thickness $D=50 \mathrm{~nm}$ is shown for a single plane wave under perpendicular angle. The partially coherent superposition with the methods of chapter 2 is shown in (b), where 2401 waves of different angles have been propagated. The photon energy was chosen to be $E=12.4 \mathrm{keV}$. The angular spectrum of the illumination was \pm 3 mrad.

The results of calculations similar to those in figure 5.6 (b) are shown in parts (c-e). Compared to the analytical approach with neglected radiative modes, the degree of coherence is lower. The red curves, that give $j(x=0, y)$ right at the entrance correspond to a sinc-function that describes the degree of coherence for the incoming angular spectrum. Clearly, several hundred micrometres of propagation are necessary until $j$ is dominated by guided modes. After several millimetres, the numerical results agree well with analytical theory. In performing such analyses, WG design can be optimised in terms of coherence; up until now, the design goal was damping of radiative modes. In ( $f$ ), iso-lines of intensity and coherence are shown for large propagation lengths behind the WG.

The analytical calculations of section 5.2 have been generalised by numerical propagation to account for radiative modes. This set-up allows for optimisation of WG length in terms of coherence filtering; including real structure effects as in $[31,38]$ or tapered WGs as analysed in [103] is also possible but beyond the scope of this thesis.

\footnotetext{
†"Interactive Data Language”, software package from Exelis Visual Information Solutions (formerly ITT VIS and RSI Research Systems, Inc.)
} 
(a) Intensity distribution, $\theta=0$
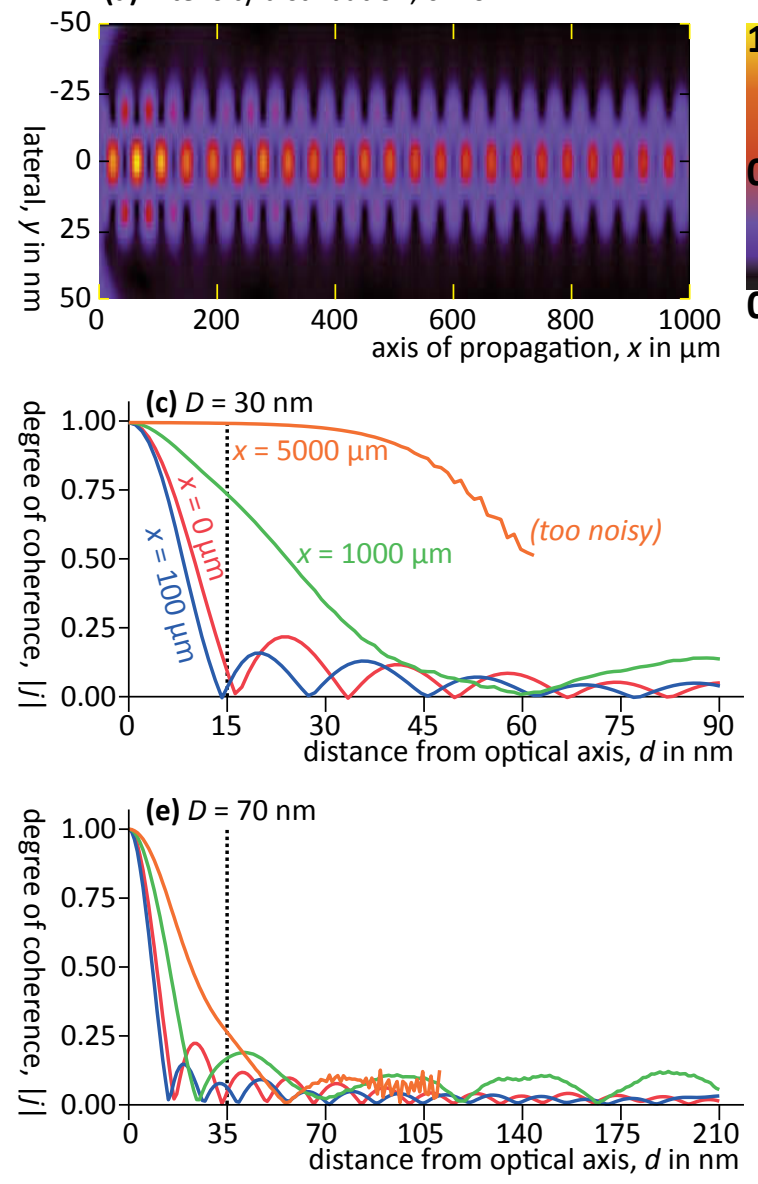

(b) Partially coherent intensity
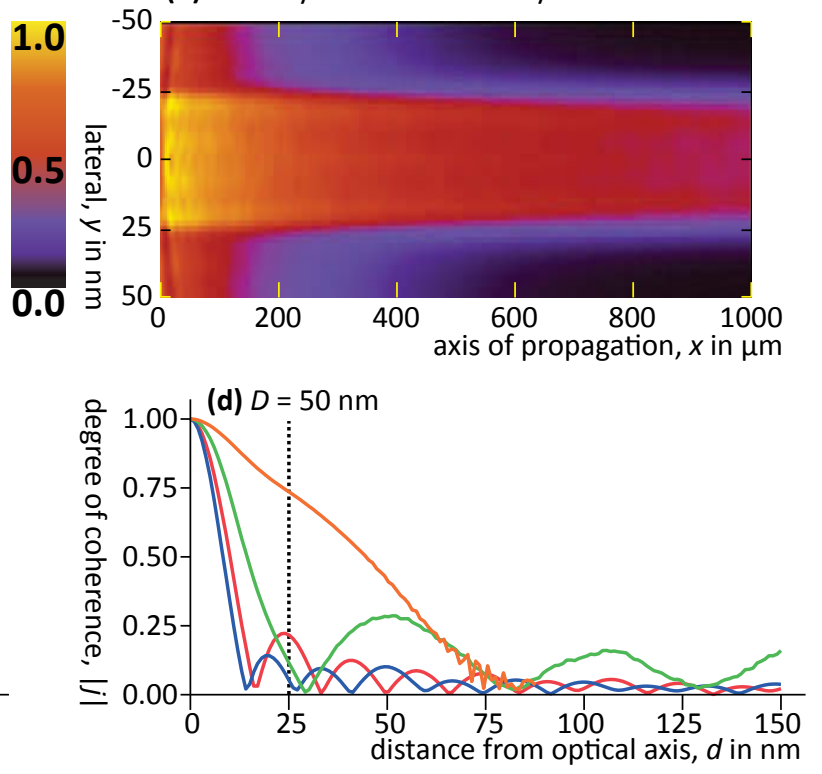

(f) Iso-lines of intensity and coherence behind the WG exit

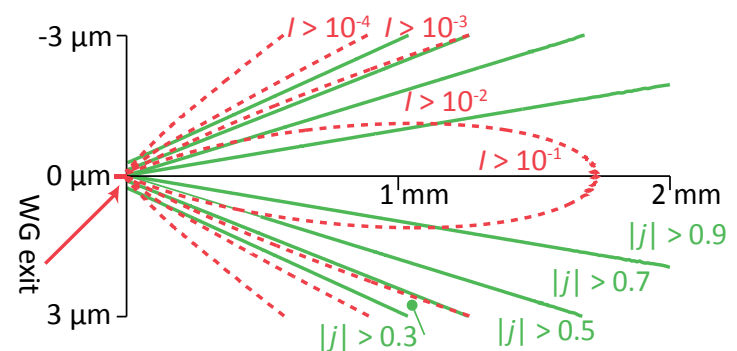

Figure 5.6: Numerically propagated degree of coherence in x-ray waveguides. In (a), coherent propagation of a single plane-wave is shown, while $(b)$ is the partially coherent intensity of 2401 waves. (c-e) show the degree of coherence in waveguides of indicated guiding layer thickness $D$ for different propagation lengths $x$. The dotted line indicates the interface between guiding layer and cladding. In ( $f$ ), iso-lines of intensity (red, dashed) and coherence (green) are shown for free-space propagation behind the WG exit. 


\subsection{Combined optics}

Up until now, the waveguide was illuminated by an angular spectrum of plane waves. In experiments, a focused $\mathrm{x}$-ray beam is used. In this section, mirror simulations taken from chapter 3 are used as an illumination of the waveguide. A Si-WG with $D=50 \mathrm{~nm}$ is placed right in the focal plane of P10-HFM that focuses x-rays of photon energy $E=7.9 \mathrm{keV}$ (or a wavelength of $\lambda=0.157 \mathrm{~nm}$ ) to a spot size of approximately $220 \mathrm{~nm}$. As can be seen from figure 5.7 (a,c, and d), the focused beam is only partially coherent. Inserting a WG (figure $5.7, \mathrm{~b}$ and e) the beam size is further reduced below $50 \mathrm{~nm}$; only after one millimetre of filtering, the beam is nearly perfectly coherent.

For propagation imaging, not only coherence is important, also beam divergence plays a crucial role to improve resolution due to larger magnifications. In figure $5.7(\mathrm{f})$ the filtered intensity and degree of coherence is shown after free-space propagation of only $100 \mu \mathrm{m}$ behind the WG's exit. Due to the strong beam confinement, a beam divergence on the order of $\sim 4$ mrad is predicted for this particular set-up, three time as high as for the focused beam.

The numerical propagation of coherence through $\mathrm{x}$-ray waveguides has been generalised to combined set-ups, where the WG is placed in the focal plane of an x-ray focusing mirror. It has been demonstrated numerically that indeed a significant beam confinement as well as nearly perfect coherence filtering is feasible with this approach. 


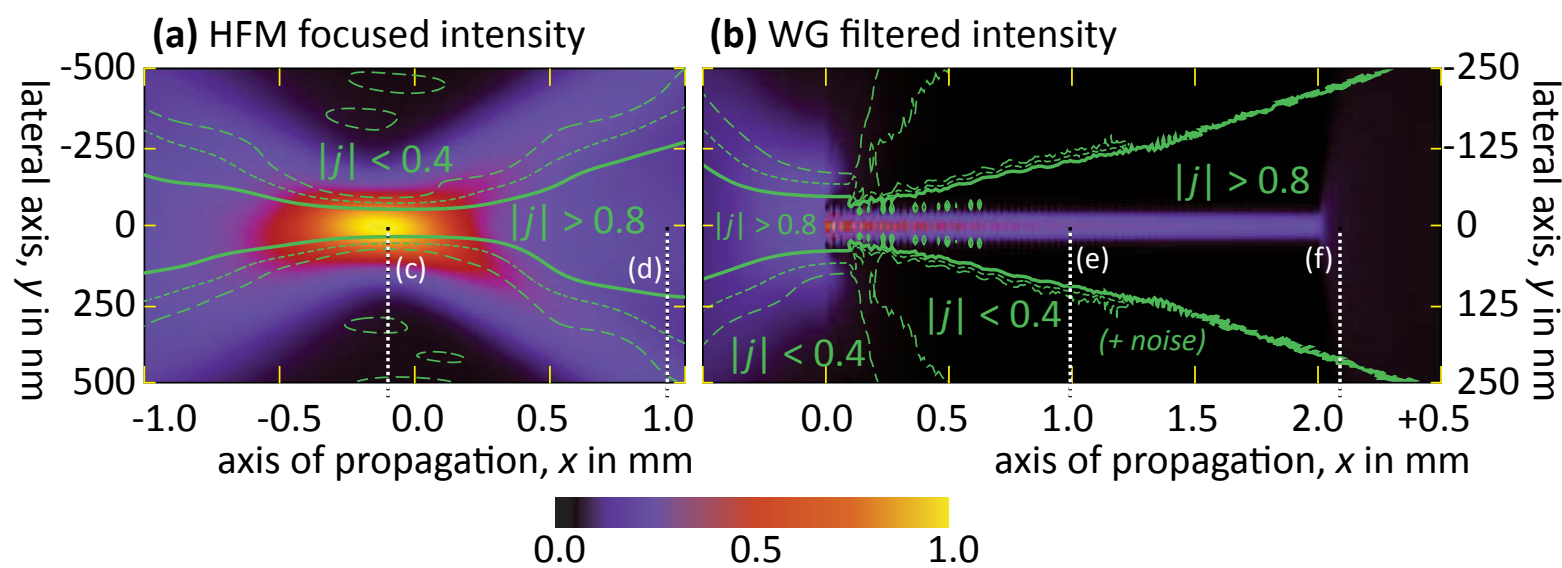

(c) Focal plane

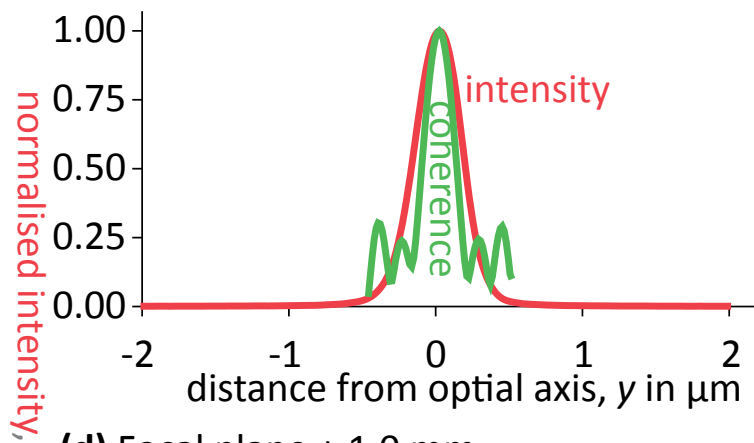

(d) Focal plane $+1.0 \mathrm{~mm}$

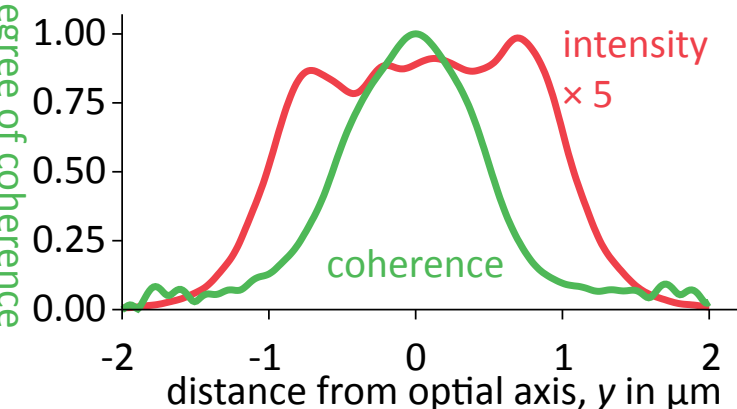

(e) Inside WG

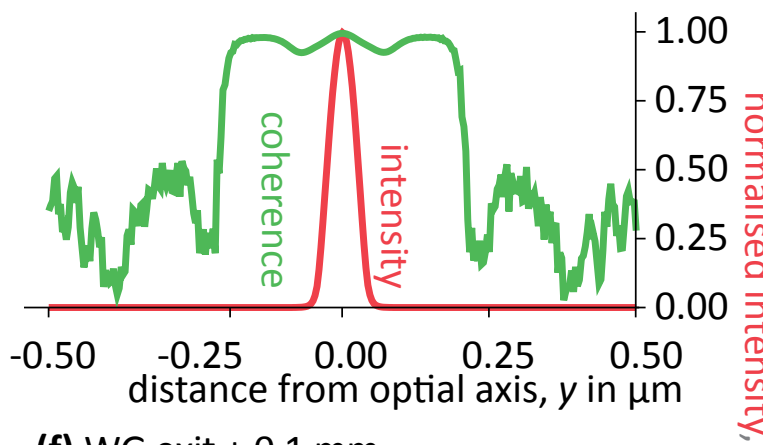

(f) WG exit $+0.1 \mathrm{~mm}$

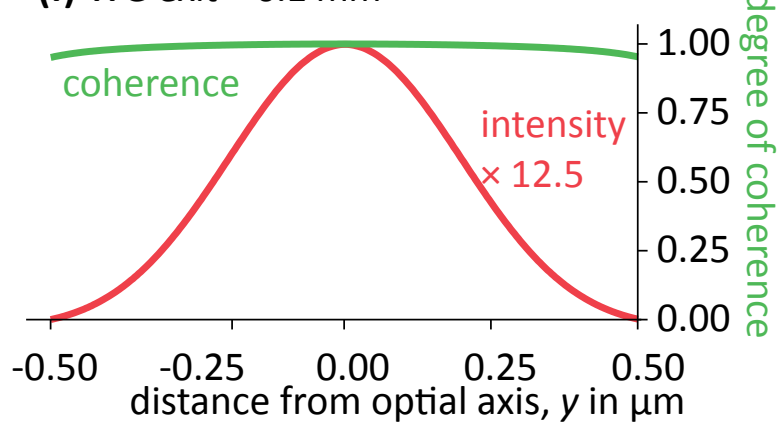

Figure 5.7: Combined optics: a waveguide placed in P10-HFM's focus plane. In (a, KB focus) and (b, WG in KB focus), the partially coherent intensity distribution (linear colour map) and iso-lines of the degree of coherence are shown. Cuts of intensity and degree of coherence along the indicated lines are drawn in (c-f). 
CHAPTER 5. WAVEGUIDES

106 


\section{Chapter 6}

\section{Conclusion}

The main goal of this thesis was to develop a wave-optical description of curved multilayer mirrors used for nano-focusing of $\mathrm{x}$-ray beams. An analytical framework has been derived, a numerical simulation programme has been written and used for optimisation. The computer codes are deployed at the European Synchrotron Radiation Facility (ESRF, Grenoble) and the Institut für Röntgenphysik, Göttingen, to assist in the design of future experiments.

More incidentally, a stochastic description of random optical fields has been used to model spatial coherence properties. Combined with related techniques, this approach has helped in the understanding of $x$-ray propagation imaging experiments. The method of stochastic fields is very flexible and can be used on top of existing software that propagates deterministic wave-fields.

\subsection{Summary}

The following subsections give a short summary of the main results found in this thesis regarding coherence and multilayer mirrors (ML mirrors). Open questions for further research and programming tasks are stated in the following section.

\subsubsection{Coherence}

Partial coherence properties of optical fields are described by their stochastic properties. Most sources, including current $x$-ray laboratory or synchrotron radiation sources, show rather strong random variations in the time- and space-domain. Random superpositions of 
pre-calculated complex valued fields, as described in chapter 2, give a descriptive and vivid representation of such random fields.

This method has been used to better understand the properties of a focusing set-up at the synchrotron source PETRA III, see subsection 3.3.4. The simulated prediction complies well with experimental data of focus size scans. Good agreement with analytical theory has been found quantitatively in section 5.3. Using this approach, coherence filtering by $x$-ray waveguides has be demonstrated theoretically.

\subsubsection{Multilayer mirrors}

A wave-optical theory describing propagation of an incoming and a reflected wave inside (nearly) elliptically curved focusing multilayer mirrors has been developed in chapter 4 . The analytical treatment in subsection 4.3.2 provides confidence in the modified Bragg condition, so far only derived on geometrical grounds. The numerical treatment of the Takagi-Taupin equations (4.7) allows for further optimisation, in terms of reflectivity and focus spot size.

From first-order approximations, diffraction limited focusing may be expected. The numerical propagation and focusing yet show deviations (see, for example, figure 4.23). But statements of focus size $\Delta$ heavily depend on the definition of $\Delta$. In this work, the full width at half maximum of a fitted $\operatorname{sinc}^{2}$-function has been chosen; the quality of the fit was not as high as expected, however.

\subsection{Outlook}

\subsubsection{Coherence}

Based on random superpositions of basic-fields, a stochastic description of partial coherence has been described in chapter 2 . So far, the equal-time spatial coherence $j\left(\vec{x}_{1}, \vec{x}_{2}\right)$ has been addressed. Incorporating the time-domain is desirable, regarding the emerging availability of short-pulse sources such as free electron lasers or plasma sources.

For simplicity, the source plane itself has been assumed to be free of any correlations. It is well-known that such correlations exist, and show a great influence, at least in the vertical direction. These might be included in the modelling scheme using non-flat distributions for the random coefficients $c_{n}$. Further generalisations might model long undulators or coupled undulator sources. 


\subsubsection{Multilayer mirrors}

As a first model, we have derived Takagi-Taupin-like equations based on the assumption of the two-beam approximation. Further effort might be reasonable, since higher-order reflections allow for larger layer spacings and smaller spot sizes.

For the numerical solution, a simple algorithm based on the finite difference method has been used. The current implementation does not allow for an adaptive step size and turns out to be rather inefficient in case of large layer spacing gradients. Appropriate step sizes have been found to depend on the largest local angle of incidence; while the transformation to elliptical coordinates is very reasonable on analytical grounds, a description based on an angular coordinate might show better numerical performance.

Without a proper description of radiation conditions at the boundary, the finite element method does not seem to be usable. It would intrinsically lead to more efficient implementations.

Some approximations used in the current approach should be questioned in further works on this topic. We list some open discussions and implementation tasks; see also subsection 4.4.3.

- vector versus scalar theory,

- boundary condition of the incoming wave-field,

- real-structure effects due to non-perfect layer deposition,
- effects due to diffusion or chemical reactions

- merging the ML code with the coherence code,

- a modified Bragg condition correcting for change of reflection angle. 
I want to close with Robert Hanbury Brown [104], describing

"the features of modern science.

It starts, like so many other branches of science, with an accidental discovery and then shows how the construction of new tools [...] brings us entirely new knowledge of the world, knowledge which is so strange that it could never have been foreseen.

It shows how our knowledge of the real world is limited by the tools which are available at the time, and goes on to show how each step forward in science depends on the interplay of imaginative theory $[\ldots]$ and experiment;

furthermore it illustrates how each discovery in an active science raises more questions than it answers." 


\section{Appendix A}

\section{Tabular derivation}

In subsection 4.2.2, Takagi-Taupin equations have been written for non-elliptical multilayers, which are described by a deviation function $\varphi(s, t)$. Elliptical MLs fulfil the Bragg condition for an incoming cylindrical wave, and can be described by a Fourier series expansion in $\exp (i 2 k t)$, q.v. (4.5) and its following paragraph. Non-elliptical MLs may be approximated using a pseudo Fourier series in $\exp (i 2 k t-i k \varphi(s, t))$. Thence the phase factor $\exp (i k \varphi(s, t))$ is introduced into the reflected wave:

$$
P_{1}^{\prime}:=P_{1} e^{i k \varphi(s, t)}
$$

A parallel derivation of the Takagi-Taupin equations for the incoming wave, the Braggreflected wave, and the modified Bragg-reflected wave is shown on the following page, along with some notes describing the steps and some terms. 

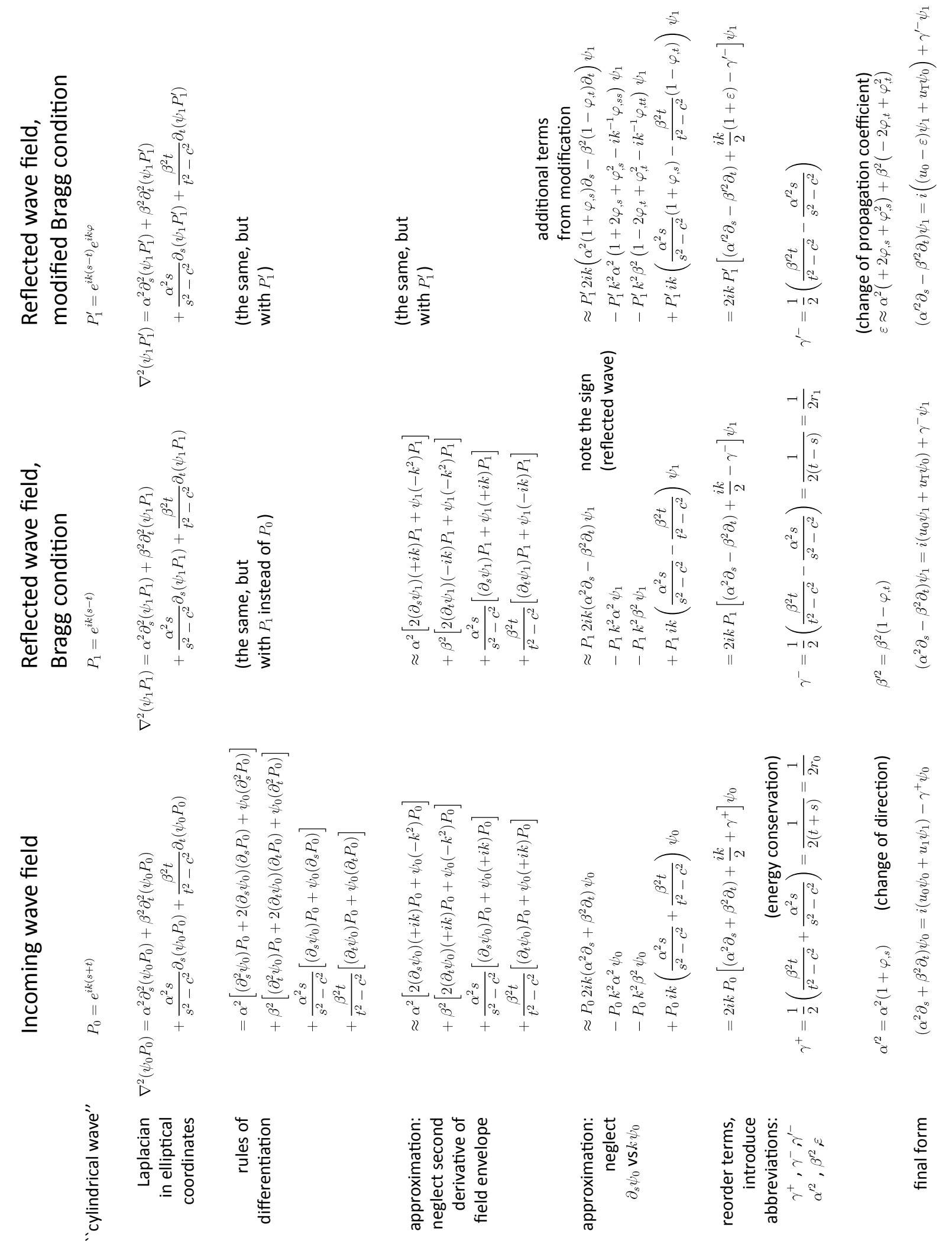


\section{Appendix B}

\section{Families of rays}

Here we give a short recursive algorithm, written in ANSI $C$, that prints families of rays, propagating inside a multilayer. The families are characterised by the number $2 n-1, n \geq 1$ of transmissions and reflections at the ML interfaces. The discrete length, the number of layers passed, of such a ray is $2 n$. The rays of family $2 n-1$ are supposed to leave the ML in step $2 n-1$ through the surface, denoted as interface 0 .

The following $\mathrm{C}$-code enumerates all rays of a given family $2 n-1$, see the example output and table B.1 for the first families. The number of rays in family $2 n-1$ is given by the Catalan number $c_{n}$.

In theoretical computer sciences and graph theory, the rays in these families are known as monotonic paths $[84,85]$. For an exhaustive discussion of monotonic paths, cf. subsubsection 2.3.4.4 in [86].

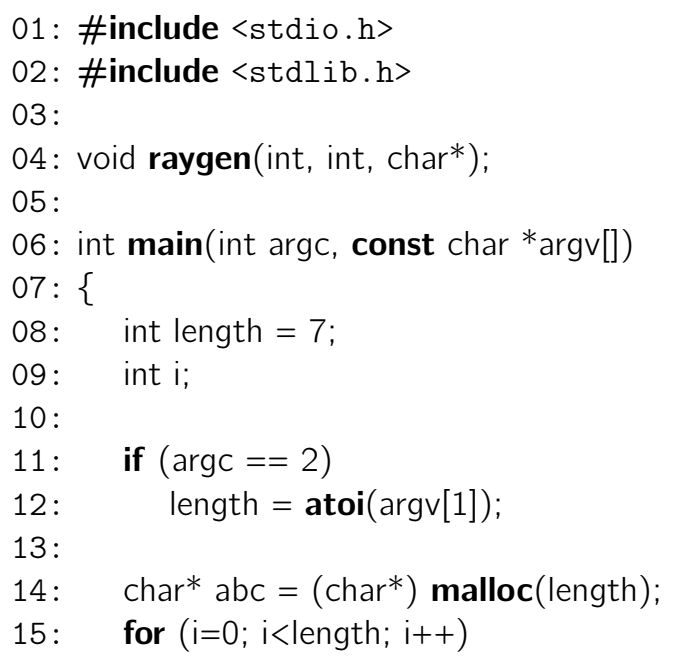

16:

17 :

18: $\quad$ raygen(length, 1, abc);

19:

20:

21:

22: $\}$

23:

24: void raygen(int length, int position, char* abc) 25: \{

26:

27:

28:

29:

30 : free $(a b c)$; return 0 ; char* abc1, *abc2; int i;

if (position $==$ length)

\{ 

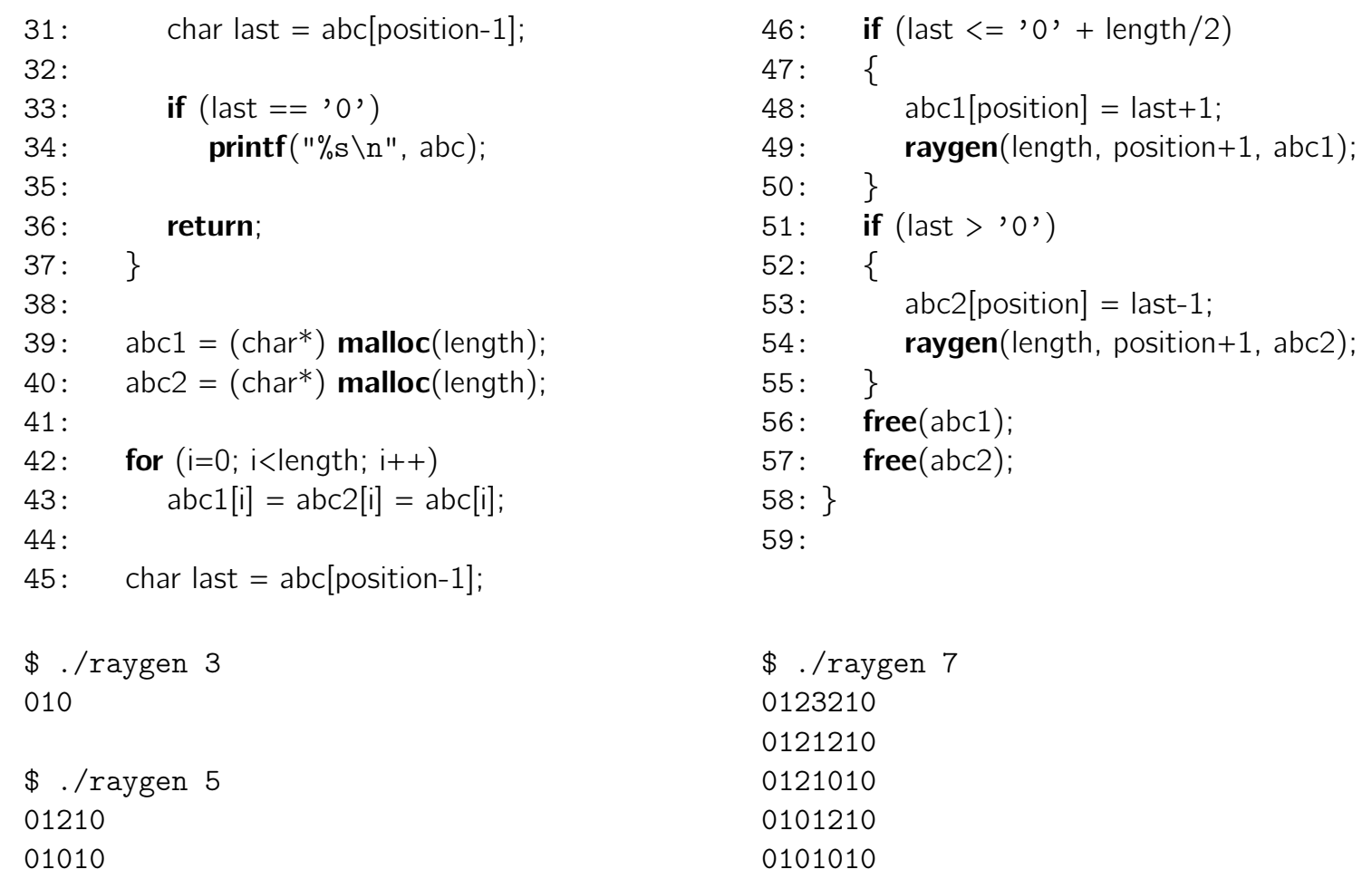

\$./raygen 7
0123210
0121210
0121010
0101210
0101010

\begin{tabular}{rrrr}
$2 n-1$ & $c_{n}$ & $2 n-1$ & $c_{n}$ \\
\hline 1 & 1 & 15 & 429 \\
3 & 1 & 17 & 1430 \\
5 & 2 & 19 & 4862 \\
7 & 5 & 21 & 16796 \\
9 & 14 & 23 & 58786 \\
11 & 42 & 25 & 208012 \\
13 & 132 & 27 & 742900
\end{tabular}

Table B.1: The number of rays within family $2 n-1$ is given as the Catalan number $c_{n}$.

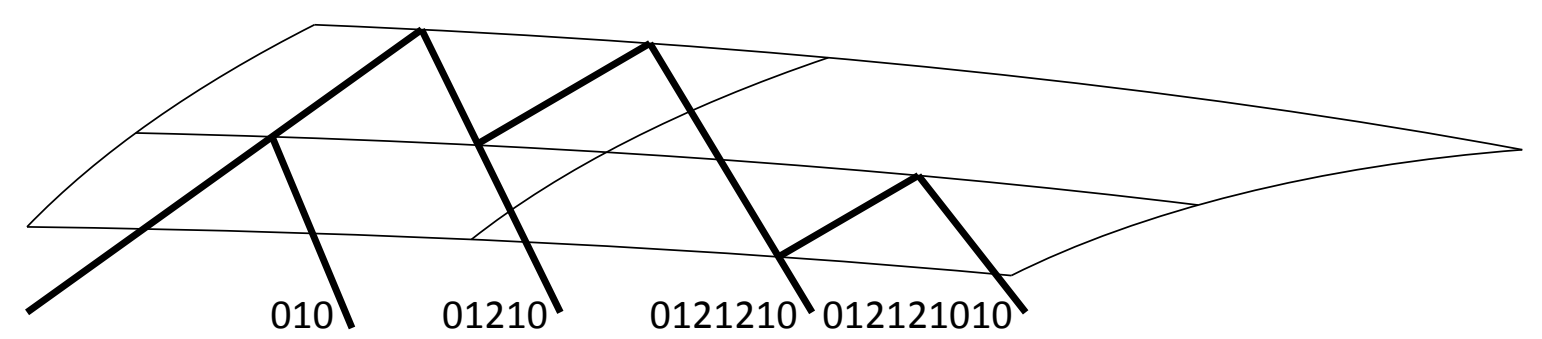

Figure B.1: Sketch to illustrate naming convention of multiple-reflections. 


\section{Appendix C}

\section{Implementation details of XMLS}

An overview of the simulation programme XMLS (x-ray multilayer mirror simulations) is given in this appendix. The following sections show an example user session, the corresponding call graph of the $\mathrm{C}++$-code, and a brief summary of the most important classes. Unfortunately, we cannot go into detail.

\section{C.1 Example session}

In this section we present an example session of running the Command Line Interface (CLI) of XMLS. Actually, a multilayer mirror is simulated; analogue output of the takagi-taupin simulation programme is skipped and shown later in subsection D.1.

User input is marked in boldface. See notes below for explanations.

This is kernel v.098e running

on htc5202.nice, Linux-2.6.18-238.9.1.el5

debug level is 1; logging

[ PROGRESS MESSAGE ERROR WARN TIMING ]

xmls \$ get geometry.s1

geometry.s1 is $1.45 e+08$

$\mathrm{xmls} \$$ get geometry.s2

geometry.s2 is 10000

$\mathrm{xmls}$ \$ get geometry.theta

geometry.theta is 0.014

xmls \$ set geometry.s1 80e6

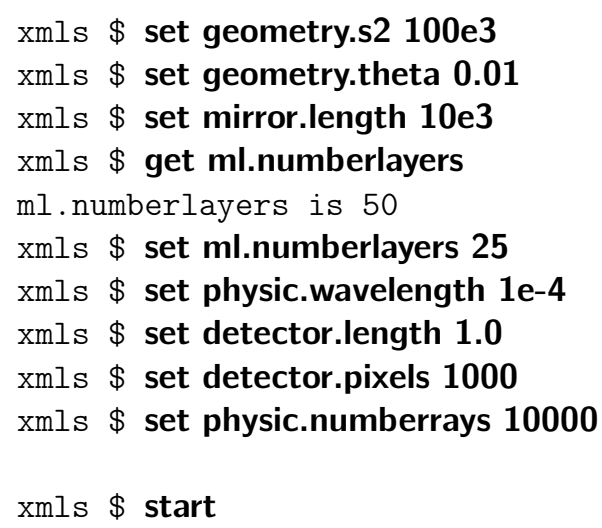


(see subsection D.1)

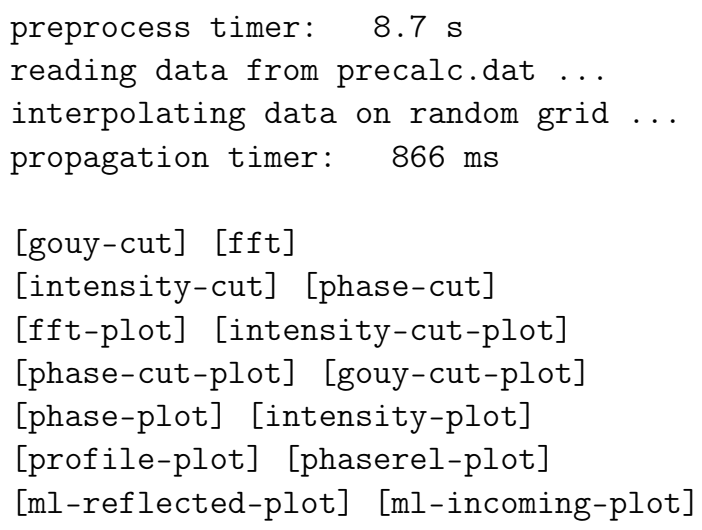

1. This is the greeting of XMLS, telling the version number, host name and Linux kernel version. The debug level is set to 1 , hence only part of possible messages are shown.

2. get <para> is the CLI command to ask for the current value of parameter $<$ para $>$. XMLS shows the value on the next line.

3. set $\langle$ para $\rangle\langle$ val $\rangle$ is the $\mathrm{CLI}$ command to set the parameter <para $>$ to the new value $<$ val $>$.

4. start is the CLI command to start a single simulation run with the current set of parameters. See section C.2 for a list of commands used to define multiple runs using loops.

5. Output of this multilayer simulation is shown and annotated in subsection D.1. The propagation timer shows the time needed for the propagation from the mirror surface to the focal region.

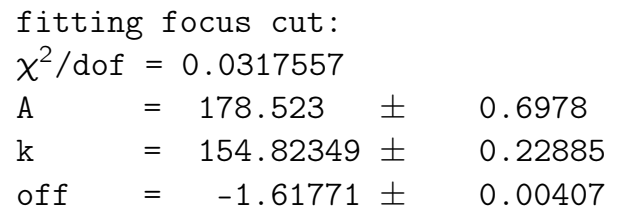

6. The class CPostprocessor creates a number of line-plots using gnuplot*, and some colour map plots with a built-in programme (but using the typical gnuplot colour maps).

7. At the end, the focus size is obtained from a $\sin c^{2}$-fit to the intensity data. First the fit parameters $A, k$, off are printed, subsequently the focus size as given by the Rayleigh criterion (distance between first minima) and as a full width at half maximum (FWHM); units are in nanometres, including error estimates as given by the fit function. As a backend for the fit algorithm, the non-linear multi-fit routines from the GNU Scientific Library ${ }^{\dagger}$ are used.

8. exit is the CLI command to end the simulation programme.

More commands available in the CLI are explained in the following section.

\section{C.2 CLI commands}

The XMLS CLI offers several commands to interactively control the simulations. The get, set, and loop commands act on the parameter set to be described in section C.3.

\footnotetext{
*http://www . gnuplot.info

†http://www.gnu.org/s/gsl
} 
$\$$ get <para> - prints the current value of the specified parameter on the next output line

$\$$ set <para> <value> - sets the given new value to the specified parameter; a loop (see next point) would be overwritten

$\$$ loop <para $><$ from $><$ till $><$ steps $>-$ defines a loop, usable in a multirun (using the command do); the given parameter will be varied in the given interval, resulting in steps+1 simulation runs; nested loops are possible

\$ start - starts a singlerun with the current set of parameters

\$ do - starts a multirun with the current set of parameters and loops; nested loops are possible; note: output files (see section C.4) may be overwritten, use hooks as described in section C.5 to rename them

$\$$ mlscan <para $><$ from $><$ till $><$ steps $>-$ is the command for a multilayer scan; here, <para> might be one of theta, rot.theta, rotation.theta, or modbragg.factor; see section C.6 for details; the parameter is looped over as in the loop command; for some parameters, simulations are started in parallel, so take care of memory and CPU load
$\$$ exit and quit - ends the programme

$\$$ run $<$ prog $>$ - starts the given shell command via the system(3) syscall

\$ restart - restarts the XMLS kernel, re-reading the xmls-defaults. conf file

\$ recompile - recompiles the XMLS source code, and if successful, restarts the kernel

$\$$ estimate - calculates a rough estimate of the focus size, using a thin lens approximation; this information may give good values for the parameter detector. length described in section C.3; also this estimate is used as a first guess for the $\operatorname{sinc}^{2}$-fit

$\$$ fit - performs a sinc ${ }^{2}$-fit to the intensity cut in the focal plane; the fit is done by the non-linear multi-fit routines form the GNU Scientific Library $\ddagger$ the initialisation is done with values from the estimate command shown above

\$ ana - calculates some experimental and rather inaccurate focus properties

\$ thetac - prints the critical angle of total reflection, $\theta_{c}$, for the current multilayer materials and the current photon energy

\section{C.3 Parameters of XMLS}

The current XMLS version knows about three dozens of parameters, that are organised hierarchically. All parameters can be set, get, and looped over, as described in section C.2. When started, XMLS reads the default parameter set from the file xmls-defaults.conf, which uses the set-syntax. We now describe the current set of parameters, sorted within eight groups.

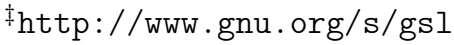




\begin{tabular}{|c|c|c|c|c|}
\hline \multirow[t]{8}{*}{ detector } & angle & & a & $\begin{array}{l}\text { angle of rotation for the detector, if optical axis is } \\
\text { misaligned }\end{array}$ \\
\hline & length & & 1 & the length of each $1 \mathrm{~d}$ detector \\
\hline & line & first & 1 & distance of first $1 \mathrm{~d}$ detector from nominal focal plane \\
\hline & & last & 1 & distance of last $1 \mathrm{~d}$ detector from nominal focal plane \\
\hline & & number & $\mathrm{n}$ & $\begin{array}{l}\text { the number of } 1 \mathrm{~d} \text { detectors, i.e. resolution along opti- } \\
\text { cal axis }\end{array}$ \\
\hline & offset & A & 1 & $\begin{array}{l}\text { offset of detector centre from nominal focal plane, } \\
\text { along } A \text {-axis }\end{array}$ \\
\hline & & $\mathrm{B}$ & 1 & $\begin{array}{l}\text { offset of detector centre from nominal focal plane, } \\
\text { along } B \text {-axis }\end{array}$ \\
\hline & pixels & & $\mathrm{n}$ & number of pixels of the $1 \mathrm{~d}$ detector \\
\hline \multirow[t]{3}{*}{ geometry } & s1 & & 1 & distance from the source to mirror's centre \\
\hline & $s 2$ & & 1 & focal distance, measured from mirror's centre \\
\hline & theta & & a & gracing incidence angle at the mirror's centre \\
\hline \multirow[t]{3}{*}{ mirror } & length & & 1 & projection of mirror length onto $A$-axis \\
\hline & reality & & f & factor scaling the height deviation profile \\
\hline & slits & & $f$ & factor to the length, to scan the effective length \\
\hline \multirow[t]{10}{*}{ ml } & rot & theta & a & angle of ML modification "rot" (see section D.3) \\
\hline & rotation & theta & a & angle of ML modification "rotation" (see section D.3) \\
\hline & modbragg & factor & f & $\begin{array}{l}\text { factor of ML modification "modbragg" (see section } \\
\text { D.3) }\end{array}$ \\
\hline & numberlayers & & $\mathrm{n}$ & nber of ML bilayers \\
\hline & layerratio & & f & ratio of the widths of the mono-layers in a bilayer \\
\hline & gridratio & & $f$ & $\begin{array}{l}\text { ratio of grid discretisation points in } s \text { - and } t \text {-direction } \\
\text { (see section D.4) }\end{array}$ \\
\hline & gridfactor & & f & $\begin{array}{l}\text { number of discretisation points in } t \text {-direction, factor } \\
\text { of numberlayers }\end{array}$ \\
\hline & factordelta & & f & factor of optical index, delta \\
\hline & factorbeta & & f & factor of optical index, beta \\
\hline & maxmem & & $\mathrm{n}$ & memory limit, in $\mathrm{MB}$ \\
\hline \multirow[t]{2}{*}{ physic } & numberrays & & $\mathrm{n}$ & $\begin{array}{l}\text { number of points on the mirror's surface to be propa- } \\
\text { gated to focal region }\end{array}$ \\
\hline & wavelength & & 1 & wavelength \\
\hline \multirow[t]{2}{*}{ postproc } & plotting & & $\mathrm{n}$ & flag, if results should be plotted \\
\hline & fit & & $\mathrm{n}$ & flag, if focal cut is to be fitted \\
\hline \multirow[t]{2}{*}{ simu } & autorun & & $\mathrm{n}$ & flag, if simulation starts after set command \\
\hline & numberthreads & & $\mathrm{n}$ & $\begin{array}{l}\text { number of threads to be used in propagation (and sev- } \\
\text { eral mlscans) }\end{array}$ \\
\hline \multirow[t]{3}{*}{ source } & divergence & & $a$ & angle of divergence of the source \\
\hline & offset & A & 1 & offset of the source, along $A$-axis \\
\hline & & $\mathrm{B}$ & 1 & offset of the source, along $B$-axis \\
\hline
\end{tabular}

The unit of lengths (indicated by an 1 in the fourth column) is micrometre, unit of angles (indicated by an a) is milliradians; factors (indicated by an $f$ ) are real numbers, whilst numbers ( $\mathrm{n}$ ) are treated as integers. For simplicity, the source code treats all parameters as double precision floating point numbers. Therefore, numbers are rounded to next integers, flags are compared to 0.5 . 


\section{C.4 Output files}

The XMLS programme writes its simulation output to several files in the fold data/; plot image files are copied into the webgui-folder, where also the HTML and related files reside.

\section{C.4.1 Data files}

The numerical data are written as ASCII coded numbers (usually floating point numbers in decimal representation, equivalent to the $C$ formatted output modifiers $\% f$ or $\% e$ ). We distinguish one-dimensional (1D) and two-dimensional (2D) data. The most important file is

- intensity.dat (2D). Here, the intensity and phase (not: amplitude and phase) is stored, for each pixel of the 2D-detector. The data are organised as a four-column table, representing (i) the optical axis, (ii) the lateral axis (both in micro metre), (iii) the intensity (in arbitrary units) and (iv) the phase (in radian; domain: $[-\pi, \pi]$ ).

- intensity-cut.dat (1D) is a cut of intensity. dat in the focal plane; it contains two columns: (i) the lateral axis (in micro metre) and (ii) the intensity.

- phase-cut.dat (1D) is similar to intensity-cut.dat, but it holds the phase in the focal plane. This file contains two columns: (i) the lateral axis (in micro metre) and (ii) the phase (in radian).

- phase-cut-unmod.dat (1D) is the same, but acted with a phase-unwrapping algorithm upon.
- gouy-cut dat (1D) is the phase along the optical axis, hence it contains two columns: (i) the optical axis (in micro metre) and (ii) the phase (in radian, where a plane-wave has been subtracted).

- farfield.dat (1D) saves the intensity for the far-field, as predicted by an FFT for, basically, intensity-cut.dat and phase-cut.dat. Note that the data is subject to large noise, even for high numbers of points propagated from the mirror's surface (physics.numberofrays should be at least $\left.10^{5}\right)$.

- scanfile.dat (1D) is the result of the mlscan command, i.e. it holds the average intensity, phase at the ML mirror's centre, and the reflected phase's standard deviation in columns (ii) to (iv); the meaning of first column (i) depends on the mlscan type, e.g. rot.theta, rotation. theta, or modbragg.factor.

\section{C.4.2 Image files}

If the parameter postproc.plotting is larger that 0.5 , at the end of each simulation run the data will be plotted. Like for the data files, we distinguish between one-dimensional (1D) and two-dimensional (2D) plots. All plots are output in Scalable Vector Graphics format 
(SVG). In the case of 2D-plots, only the axes and labels are saved as vectors, while the actual data are plotted to linked Portable Network Graphics (png) files, hence bitmaps.

The 1D-plots and the SVG-skeleton including axes of the 2D-plots are generated by Gnuplot, while the 2D colour maps are produced by the programme plot.cpp. The plots can be found in the webgui/folder.

- intensity-large.svg (2D, +.png) represents the intensity distribution in the focal region.

- phasemap-large.svg (2D, +.png) represents the phase in the focal region.

- phaserel-large.svg (2D, +.png) is the same, but with a plane-wave subtracted.

- plot-mini.png (2D) is a low resolution version of intensity-large.png, used as a preview in the web GUI.

- phasemap.png (2D) is a preview of phasemap-large.png.

- phaserel.png (2D) is a preview of phaserel-large.png.

- focuscut_int.svg (1D) is a plot of intensity-cut.dat in the focal plane.

- focuscut_phs.svg (1D) is a plot of phase-cut.dat in the focal plane.
- focuscut_phs_unmod.svg (1D) is a plot of phase-cut-unmod.dat, the unwrapped phase-cut.dat.

- gouy.svg (1D) shows the Gouy phase of gouy-cut.dat.

- farfield.svg (1D) shows the far-field as obtained by a simple FFT§.

- ml_incoming_int.svg, ..._phs.svg (1D) show the intensity and phase of the incoming wave-field onto the ML mirror, while

- ml_reflected_int.svg, ..._phs.svg (1D) are for the reflected wave-field. Furthermore,

- scan_1.svg, scan_2.svg, scan_3.svg (1D) show the reflectivity, phase, and standard deviation of the reflected phase, of the last mlscan run.

\section{C.5 Hooks}

In section C.2, the CLI command loop was described; it allows for a multirun over (possibly nested) loops of large parameter spaces. But each single simulation would override former data and plot files. Using hooks, it is possible to run bash scripts after different steps of the simulation programme. There, environment variables can be accessed to move or rename files according to the parameter values. Hooks also allow for a flexible junction to other numerical tools, like more sophisticated plotting or fitting routines. The following hooks are available; if files of those names exist and are executable, they will be executed at the indicated steps within the simulation programme:

\footnotetext{
$\S$ http://www.fftw.org/
} 
- theta scans the gracing angle of incidence on the ML mirror's centre; note, that each simulation is described by a different ellipse.

- rot.theta is a scan of the modification function rot (see subsection D.3.1), representing a simple rocking curve of the $\mathrm{ML}$ mirror.
- rotation. theta does the same, but using a more complicated description of the rotation, see subsection D.3.2).

- modbragg is a pseudo-rocking curve, scaling the modification of Bragg's law for refraction; see subsection D.3.3.

All these commands write their output to the file scanfile.dat with an appropriate heading. There is one column for the scan value, and three for data: the integrated or average reflectivity of the ML mirror, the phase at the centre, and the standard deviation of the phase along the surface. Plots are generated to scan_1.svg, scan_2.svg, and scan_3.svg, corresponding to the three data columns of scanfile.dat.

\section{C.7 Call graph}

The whole XMLS suite consists of more than two hundred $\mathrm{C}++$ and $\mathrm{C}$ code functions. Obviously, we only can focus on a small number in this thesis; the main topic is physical interpretations of the simulations. To assist future updates of the code base, we give a very brief overview the XMLS call graph - what happens, if the user wishes new results. This is sketched in figure C.1.

The main (...) function resides in kernel.cpp and spawns several interfaces; a new simulation is started inside the callback (...) function. There an instance of CKernel is created and control delegated via $\operatorname{simulate}(\ldots)$ and postprocess (...) functions"l.

CKernel creates instances of phyiscal objects, like CPSource that models a monochromatic point-source, CPMFresnelReflectivity for a simple mirror described by a tabulated index of refraction, and CPDLine, a 1D-detector. Some steps are influenced by the class CSimulation. After calculating the illumination function and applying Fresnel's coefficient of reflectivity, the line detector evaluates Kirchhoff's diffraction formula and writes the propagated field to disk. An instance of CPostprocessor then generates line cuts of the two-dimensional data and runs external programmes for plotting.

\footnotetext{
$\|$ In case of the multilayer simulation running as a separate programme, there is also a preprocess (...) function that starts the Takagi-Taupin code described later.
} 


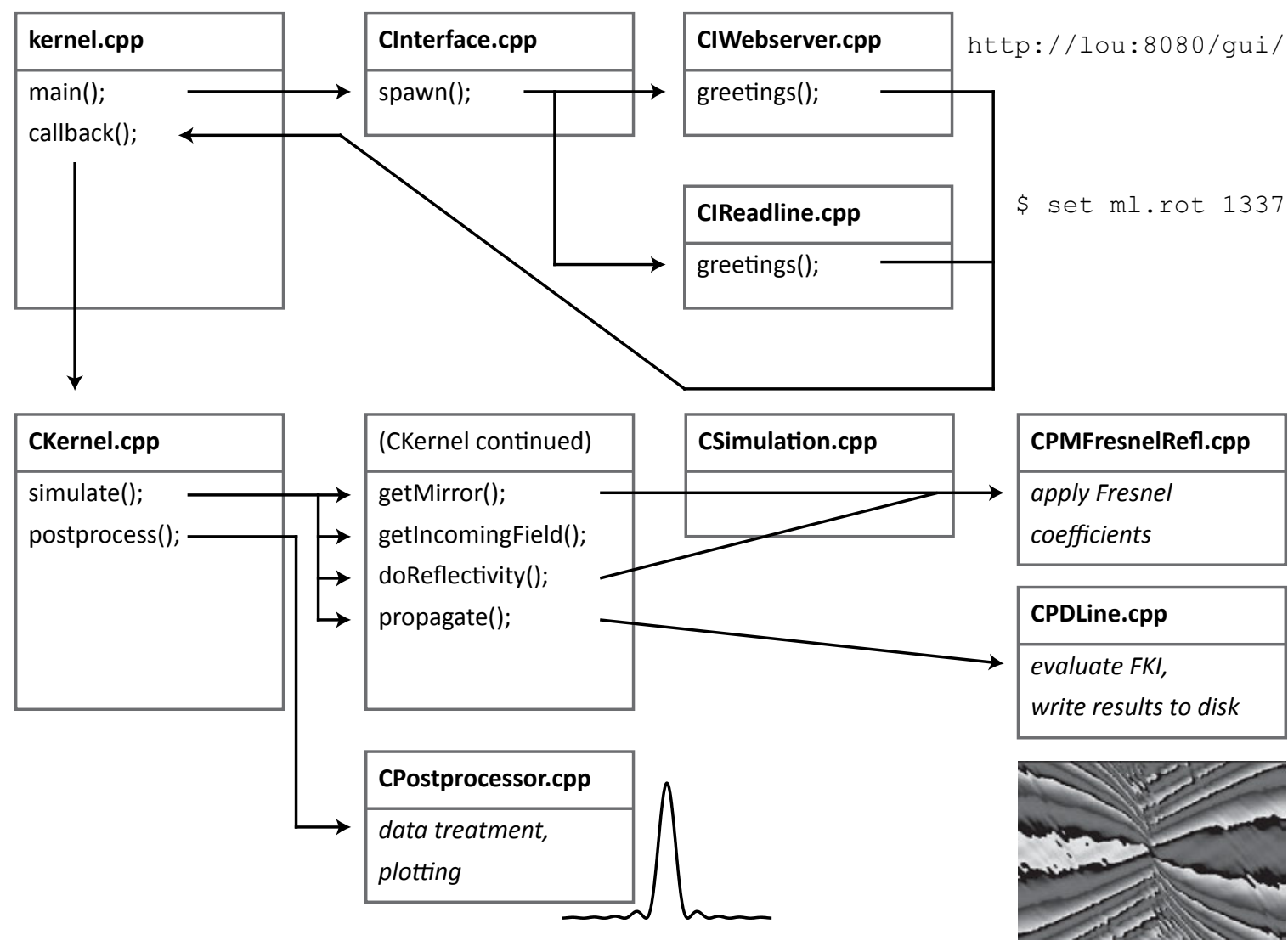

Figure C.1: Very incomplete illustration of the call graph within XMLS; this is just an overview due to space constraints.

\section{C.8 Physical classes}

In this section we have a look at important classes of the XMLS source base that model physical objects like a Fresnel mirror and a detector (that does the actual free-space propagation). The following section C.9 then describes additional helper classes extensively used within XMLS.

\section{C.8.1 CPMFresnelReflectivity}

This class models a Fresnel mirror; it gets the complex valued illumination function from a source and applies Fresnel's coefficient of reflection. Although elliptically curved, the mirror is assumed to be locally flat, so the Fresnel coefficients for plane-waves and flat surfaces are 
used. Currently, the coefficient for the $\sigma$-component (the electrical field vector oscillates perpendicular to the surface) is used. Comparing (3.2) and (3.3), this is justified: For gracing incidence angles below some hundred milliradians the reflectivities are virtually the same.

Fresnel's coefficient (3.2) is written in terms of incoming $\theta_{1}$ and refracted $\theta_{2}$; the latter therefore is calculated from Snell's law (3.1). Using complex variables, the phase change (3.4) is accounted for. Since $\mathrm{C}++$ does not come with a complex variant of the inverse cosine, this is represented using a logarithm:

$$
\cos ^{-1}(\phi) \equiv-i \log \left(\phi+i \sqrt{1-\phi^{2}}\right), \quad \phi \in \mathbb{C}
$$

For a discussion of the local flux density, see subsection C.8.3.

\section{C.8.2 CPMFromFile}

The pseudo mirror CPMFromFile reads the complex valued reflected field from a file called precalc.dat, consisting of three columns: (i) the position along the mirror surface, measured in micro metre; (ii) the reflected intensity; (iii) the reflected phase in radian. The data are read and extrapolated onto a non-regular grid to avoid artefacts.

The file precalc. dat is assumed to stem from the Takagi-Taupin simulation programme discussed in appendix D. Because the field envelopes there are calculated with a cylindrical wave term omitted, the spherical wave converging to the focus is projected onto the elliptically curved mirror surface and then added to the field. This is done in the function CPMFromFile: :doReflectivity().

\section{C.8.3 CPDLine}

The "line detector" propagates the complex valued field from the mirror surface onto a one-dimensional line in the focal region. The free-space propagation is modelled using the diffraction integral of Fresnel and Kirchhoff, (3.6); this integral is evaluated using Riemann sums. The 1D-detector can be moved along the optical axis; indeed, respecting the parameters detector.line.first, detector.line.last, and detector.line.number, a parallel loop using openMP is carried out. Afterwards, the intensity and phase are written to the file intensity. dat.

For optimisation, the code in this class not only uses the openMP standard to be run in parallel, but also makes use of SSE intrinsics (if built with macro SSE defined, as in $\mathrm{g}^{++}$ 
-DSSE). These intrinsics allow usage of specialised SIMD registers in the CPU: SIMD stands for single instruction, multiple data. Hence it is possible, i.e., to calculate the square root of two numbers in parallel.

We will report on tests with different compiler versions, GCC 4.1 and GCC 4.3, in appendix J. Experience strongly suggests to use GCC 4.3 or newer.

A remark on best practices using openMP: It is recommended to parallelise the outer loop, here the loop over different line detectors; if only a single cut ("only the focal plane") is to be simulated, the corresponding pragma directive should be placed in front of the "middle loop" (the loop over detector pixels).

The propagation is modelled using the scalar Fresnel-Kirchhoff diffraction integral (FKI) (3.6). Its physical foundation is the principle of diffracted elementary spherical waves following Huygens; due to its descriptive interpretation and fast numerical implementations, it is one of the standard tools in computational optics. A lot of textbook space is reserved to the discussion of the FKI - but also a lot of criticism has been raised. In the derivation, Kirchhoff had to introduce somehow disputable boundary conditions for the light field behind a diffracting screen $[3,105]$; this question has been addressed by Arnold Sommerfeld [58]. Moreover, the $\mathrm{FKI}$ is derived as a solution to the wave equation (WE); but a solution to the WE is not necessarily a solution to Maxwell's equation, as pointed out in [59].

For simplicity, an obliquity factor to be found in the FKI often is dropped, "because it is unity". This term can be interpreted as ensuring the local flux density on the curved mirror's surface: Since the angle of incidence varies along the surface, the local "strength" of both illumination and reflection changes. In CPDLine this has been accounted for by a correction factor; a similar expression, discussed for the case of parabolic mirrors, can be found in [83].

\section{C.9 Helper classes}

Much functionality, mainly for managing information and processing data, has been put into helper classes; some of them are described now.

\section{C.9.1 CKernel}

The CKernel is like the control centre of the simulation programme, where single pieces are combined together. Some of these single pieces are the illuminated mirror and the CPDLine detector carrying out the propagation; plus routines for pre- and post-processing 
like plotting.

\section{C.9.2 CSimulation}

The class CSimulation hides some aspects from CKernel, like the type of mirror to be simulated or the type of detector (if there would have been more than only CPDLine). While it is the task of CKernel to control the instances of different classes (illuminate the mirror, do the reflection, start propagation to the detector), CSimulation creates the instances.

\section{C.9.3 CPostprocessor}

The helper class CPostprocessor is mainly used as a generalised interface to call the actual plotting routines. Most of the functions just directly call Gnuplot or the plot.cpp programme; sometimes simulation parameters are incorporated in the calls to external programmes, like the number of pixels. The plotting functions are called from CKernel:: postprocess (), for efficiency made parallel with openMP.

\section{C.9.4 CFos}

This class stores the Field on the surface of the mirror. It is a container class saving the position in different coordinate systems (along the mirror, the $A$ - $B$-coordinates of the ellipse), and the amplitude in different representations. Objects of type CFos are propagated from the source to the mirror's surface.

\section{C.10 Interface classes}

In this section we describe three classes to store and handle simulation parameters. Here, interface class does not refer to the concept of abstract classes, but is meant as a class implementing a user interface. 


\section{C.10.1 CInterface}

The class CInterface stores the current values of all parameters used by XMLS. Other classes obtain their values via double CInterface: : getValue (std: :string). This class also implements the logic to traverse the nested loops for a multirun. The user interfaces, however, are not implemented here, but in the following classes CIReadline and CIWebserver.

\section{C.10.2 CIReadline}

The most robust user interface of XMLS is implemented using the GNU readline library, featuring a command line prompt with tab-completion. Here, using the commands summarised in section C.2, all parameters of section C.3 can be changed, and (possibly nested) loops can be defined, as well as external programmes started via run <prog>. There is, however, not much visualisation possible, except for some rudimentary terminal based Gnuplot output.

It is reasonable to start the XMLS programme within a GNU screen environment. That way it is possible to detach from a running session and reattach later to change parameters. Using screen $-\mathrm{xR}$, the same instance can also be controlled from different users and terminals in parallel.

\section{C.10.3 CIWebserver}

As a graphical frontend to XMLS, a novel approach featuring a web interface has been chosen. Within CIWebserver, the HTTP web server library mongoose ${ }^{* *}$ is used to communicate with other tools. This allows for a unified interface, in principle with world-wide access, to (i) set parameters, (ii) start the simulation, and (iii) obtain plots and data for further analysis. For convenience, a graphical user interface running in a web browser has been set-up using HTML, CSS and Javascript/AJAX. For more details and advantages of decoupling the simulation programme from the GUI, see appendix F. Example code fragments that allow different third-party software to control XMLS are shown in appendix G.

\footnotetext{
${ }^{* *}$ http://code.google.com/p/mongoose/
} 


\section{C.11 Current limitations of XMLS}

Here we list some limitations and problems of XMLS; those marked with a dagger $\left({ }^{\dagger}\right)$ only apply for the multilayer version.

- Number of points to be propagated: may not exceed some $10^{5}$ due to memory problems (physics. numberofrays).

- Elliptical parameters, $S_{1}, S_{2}$, and $\theta$, are not updated after first simulation run; xmls has to be restarted.

- ${ }^{\dagger}$ Height deviation profile not respected for multilayers.

- $\dagger$ Source offset not respected for multilayers.

- $\dagger$ Webgui does not report on progress for Takagi-Taupin simulation.

- ${ }^{\dagger}$ Errors in Takagi-Taupin simulation are not reported, instead mirror simulation uses old data to propagate. 
1. In the first block, optical components and constants of the ML mirror are printed. The optical constants are taken from a database, see section I.

2. The second block summarises the geometrical parameters. Values in boldface are input obtained from the calling XMLS instance via environment variables or command line arguments. For a discussion of the different modification functions rot, rotation, and modbragg, see section D.3. Important output values are the local angles of incidence at the "left" and "right" edge of the ML mirror.

3. In the layer information, the bilayer thickness at the mirror's centre, using Bragg's law (4.12), is shown, together with the thickness of the whole structure.
4. The simulation information block tells the user how many grid points are used in the discretisation of the computational domain, and how much virtual memory is occupied by the programme. A limit on the allowed memory usage may be introduced by the command line argument - -maxmem $=\langle$ value $>$.

5. The progress bar is updated while the integration is running.

6. After the integration is carried out, a small but informative on-line analysis subroutine gives a brief overview of the reflectivity, reflected phase, and the standard deviation of the phase along the surface.

7. At the end, the user time needed for the simulation run is printed.

\section{D.2 Integration routines}

Here we discuss the integration routines used to solve the Takagi-Taupin equation system (4.7). The partial differential equations are solved using a finite difference scheme and a Runge-Kutta-algorithm (RK) of second order. The algorithm is implemented in the GNU Scientific Library (GSL)* Tests with RK of fourth order and a Runge-Kutta-Fehlberg method did not show more accurate results, but were slower by a factor of three to four.

The Takagi-Taupin programme can be divided into three steps: (i) initialisation and input, (ii) solution of the TT system, and (iii) output and clean-up. In the following we summarise the second part, represented by the function int ttsolver(). But first we discuss the principle of operation.

\section{D.2.1 Principle of operation}

The TT system (4.7) consists of two coupled partial differential equations for two complexvalued envelope functions $\psi_{0}$ and $\psi_{1}$ in two dimensions, $s$ and $t$. The equations are homogeneous, but coupled via the coefficients $u_{ \pm 1}$, and show a propagation term $u_{0}$. From the physical set-up and the modelling of the two beams it is clear that the incoming beam $\psi_{0}$ is supposed to propagate in the "top-right" direction (increasing $s$ and $t$ ), while the reflected

\footnotetext{
*http://www.gnu.org/s/gsl
} 
(a) Discrete computational grid

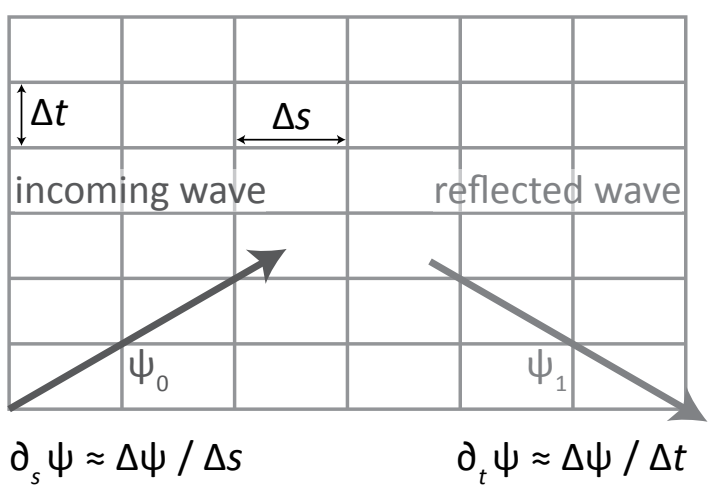

(b)
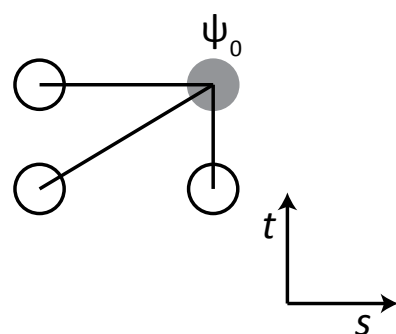

(c)

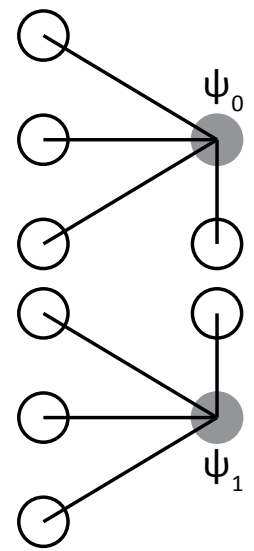

Figure D.1: Illustration of the integration scheme for the Takagi-Taupin equation system (4.7). In (a), the principle of the used model is shown. The partial derivatives are approximated by finite differences of the four-point stencil in (b). Earlier versions used the five-point stencil from (c), but this violates energy conservation, since information can travel "backwards".

beam $\psi_{1}$ is supposed to travel to "bottom-right" (that is, increasing $s$, decreasing $t$ ). As outlined in section 4.4 , the values of $\psi_{0,1}$ are known to be zero on the "left" surface, the reflected beam $\psi_{1}$ vanishes on the "top" boundary, the incoming beam $\psi_{0}$ is given by the illumination on the "bottom" boundary.

The computational domain, transformed to elliptical-hyperbolic coordinates, is divided into a "rectangular" grid of step size $\Delta s \times \Delta t$. Now we approximate the partial derivatives $\partial_{t} \psi$ of (4.7) as finite differences:

$$
\partial_{t} \psi_{0}(s, t) \approx \frac{\psi_{0}(s, t)-\psi_{0}(s, t-\Delta t)}{\Delta t}, \quad \partial_{t} \psi_{1}(s, t) \approx \frac{\psi_{1}(s, t+\Delta t)-\psi_{1}(s, t)}{\Delta t} .
$$

Note the asymmetrically chosen differences, discussed later.

If we know $\psi(s)$ on one vertical cut in the domain, both the right-hand side of (4.7) and, using the aforementioned finite difference approximation, half of the left-hand side is known. Solving for $\partial_{s} \psi$, the two envelope functions can be propagated "to the right". This scheme is illustrated in figure D.1 $(a, b)$. The integration along s-direction is carried out using the GSL RK-method.

The integration method is based on the four-point stencil shown in figure D.1 (b). In a first version, a symmetric difference of step size $2 \Delta t$ had been chosen; but this sometimes resulted in reflectivities greater than unity, hence energy was not conserved. The corresponding five-point stencil in figure D.1 (c) shows that in this case information (and hence energy) can travel "backwards" relative to the propagation direction of the two beams.

The implementation of the integration method is discussed in the following subsection. 


\section{D.2.2 Physics in source code}

In this subsection we describe the solution scheme in terms of involved C-code functions. After some initialisation steps invoked from the int main(int, char**) function, the solution starts by calling the int ttsovler() function.

int ttsolver() This function initialises the GSL RK library and carries out the loop in the propagation direction s. For each vertical slice in the computational domain, an array double $\mathrm{y}[]$ is prepared, holding the data of the previous slice. The GSL RK library then calls the function int func_P(double, double, double, void*) that provides the finite difference of the RK step in s-direction. These finite differences are copied to the array double $f[]$. The calculation of these differences is delegated to the functions setDpsi0 and setDpsi 1 which hold the proper constants for the finite difference scheme.

int func_P (...) is called from the GSL RK library to calculate the finite difference in the $s$-direction. A call to int copy_amplitudes_from_y (...) first reassembles the complex values of $\psi_{0}$ and $\psi_{1}$ from the double y [] array (this is necessary due to the GSL library). The differences are calculated by the function int get_coefficient___for_Dpsi(...), that basically implements the four-point stencil from figure D.1 (b). The stencil values are used in the functions int $\operatorname{setDpsi0}(\ldots)$ and int $\operatorname{setDpsi1}(\ldots)$.

int setDpsi0,1(..) obtain the stencil values of figure D.1 from the function int get_coefficients_for_Dpsi(...) and combine them with the right-hand-side of the TT equations to approximate the finite differences in s-direction. Special care is taken at the "top" and "bottom" boundary, where the stencil and hence the coefficients differ.

int copy_derivatives_to_f(...) Finally, this function disassembles real and imaginary part of the s-differences and puts them into the double $f[]$-array, as needed by GSL. The function int func_P ( . . . ) returns the constant GSL_SUCCESS to the GSL RK stepper function.

int ttsolver() saves the calculated values of the current slice of $\psi_{0}$ and $\psi_{1}$ to memory and continues with the next slice. A progress bar is implemented. 


\section{D.3 Modification functions}

Modification functions model geometrical strains in the $\mathrm{ML}$ mirror, introducing additional phase factors in the TT system (4.7) as described in subsection 4.2.2 and section 4.3. In XMLS, two kinds of modifications are implemented: rotations of the $M L$, resulting in a rocking-curve, and the modified Bragg condition of (4.13). The rotation is modelled using two different approximations and is described by the model functions "rot" and "rotation". The model function for the modified Bragg condition is "modbragg".

The model functions are defined as $\mathrm{C}$ macros in the file params. $\mathrm{h}$; the macros are called PHI_[ST]_[123], where $S$ and $T$ stand for the coordinates $s$ and $t$, whilst $1,2,3$ number the different models. The macros PHI_ [ST] then are defined as sums over the three model functions; these macros are used in the Takagi-Taupin integration scheme.

In subsection 4.2.2, the modified TT equations (4.9) is derived, introducing a deviation parameter $\varepsilon=\varepsilon\left(\varphi_{, s}, \varphi_{, t}\right)$; the quantities $\varphi_{, s}$ and $\varphi_{, t}$ are derivatives of the transformation function $\varphi(s, t)$ with respect to the coordinates $s$ and $t$. This $\varphi(s, t)$ is introduced to generalise the Fourier series expansion of the susceptibility $\chi(s, t)$. As shown, its derivatives contribute to the deviation parameter $\varepsilon$, which appears in the second equation of the TT system.

In the following subsections, the implementations of rotations (for rocking curves) and the modified Bragg condition (for optimisation of reflectivity and focus size) are described.

\section{D.3.1 rot}

As illustrated in figure 4.6, a rotation of the $M L$ structure relative to the incoming beam can be modelled as the original multilayer with increased layer thickness; this, on the other hand, can be modelled using a transformation of the $t$-coordinate. Depending on the direction of $M L$ rotation, the $t$-coordinate is either stretched (if the new angle of incidence is smaller than the original angle) or shortened. This transformation, that depends on coordinates and local angle of incidence, gives rather complicated terms for $\varphi_{, s}$ and $\varphi_{, t}$. In the model rot, only the most important term is used:

$$
\varphi_{, s}=0, \quad \varphi_{, t}=\Delta \theta / \beta(s, t),
$$

where $\Delta \theta$ is the rocking angle and $\beta(s, t)=\sin \theta(s, t)$ a scaling factor originating in the Jacobian of elliptical-hyperbolic coordinates. In the derivation of this form, the dependence of $\beta(s, t)$ on the coordinates has been neglected. 


\section{D.3.2 rotation}

Derivatives of $\beta(s, t)$ enter in the derivatives of $\varphi(s, t)$ by application of the chain rule. The next leading terms are included in the model function rotation, defined as

$$
\begin{aligned}
\varphi_{, s} & =-\frac{\left(t-t_{0}\right) \Delta \theta s}{\left(t^{2}-s^{2}\right) \beta}, \\
\varphi_{, t} & =\frac{\Delta \theta}{\beta(s, t)}+\frac{\left(t-t_{0}\right) \Delta \theta \alpha^{2} t}{\left(t^{2}-s^{2}\right) \beta^{3}} .
\end{aligned}
$$

Again, $\Delta \theta$ is the (global) angle of rotation, $\alpha$ and $\beta$ stem from the Jacobian and depend on position.

\section{D.3.3 modbragg}

The modified Bragg condition (4.13) changes the layer spacing to correct for refraction. Again, this modification can be interpreted as an additional transformation of coordinates (from rectangular to elliptical to "modified elliptical"). As shown in subsection 4.3.2, this transformation can be modelled as a "local rotation" $\Delta \theta(s, t)$. This local angle is given as $\delta / \beta(s, t)$, with $\delta$ as the deviation from unity of the index of refraction, $n=1-\delta$. The model function modbragg is defined like rotation, setting

$$
\Delta \theta=f \delta / \beta
$$

where the factor $f$ is a scaling parameter that can be changed for optimisation. Since now $\Delta \theta(s, t)$ also depends on position, additional terms occur due to the chain rule. A numerical estimation showed them to be small, hence they have been neglected.

\section{D.4 Calculation grid}

The computational domain inside the ML structure is discretised according to figure D.1. But how to choose the grid sizes $\Delta s \times \Delta t$ ? Usually, a fine grid is needed to approximate infinitely small derivatives well by finite differences; but this enhances numerical problems (ratio of very small numbers) and increases computational time significantly. For $\Delta t$, several tests have been done to characterise the algorithm's needs. An example is shown in figure D.2, for analogue parameters as in figure 4.10, but 30 layers. In part (b), a zoom-in to the peak of the reflectivity curve (a) is shown. 
(a) $12.4 \mathrm{keV}, 30$ layers

(b) Zoom-in
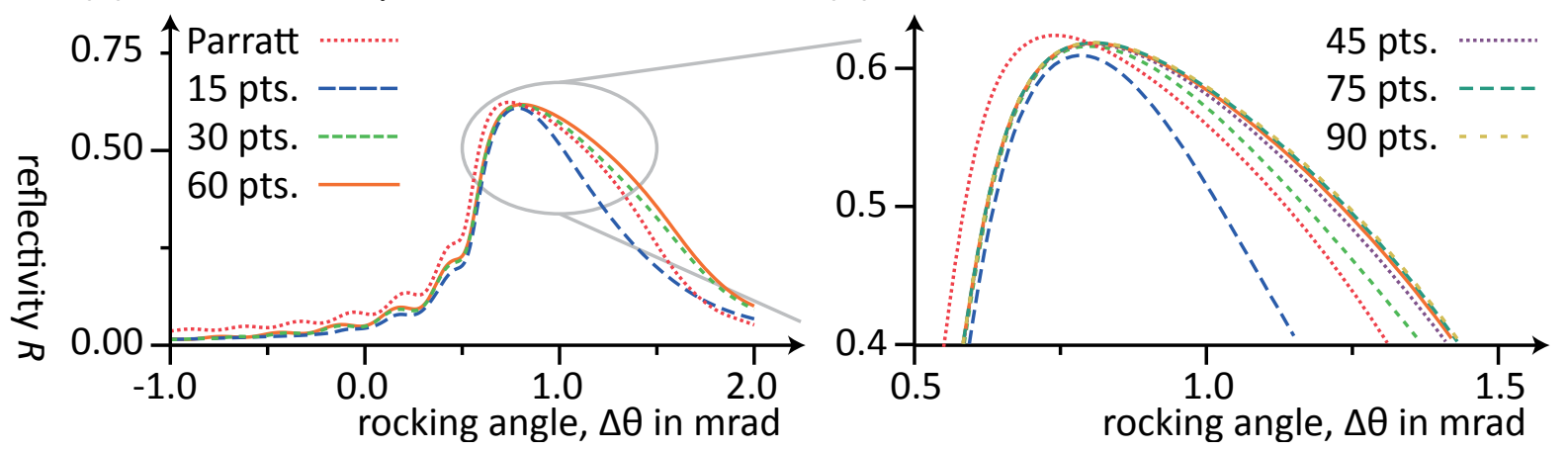

Figure D.2: Reflectivity curve analogue to figure 4.10, but with 30 layers and differing numbers of discretisation grid points in t-direction.

The curves have been obtained for different numbers of computational "layers", from half the number of actual bilayers to thrice as many. The red dots are calculated using IMD's implementation of Parratt's algorithm.

The reflectivity curves seem to converge with increasing number of grid-points; this and similar simulations suggest that one grid point per mono-layer gives reasonable results.

For the s-direction, the situation is more complicated. Tests have shown that the grid size has to be small enough, otherwise the computation results in "exploding" amplitudes. A quantity called "grid ratio" has been defined in (4.21):

$$
\mathrm{GR}=\text { gridratio }:=\theta \times \Delta s / \Delta t
$$

Tests have shown that GR $\lesssim 100$ should be used for moderate angles of incidence, $\theta \sim$ 10 mrad; but much smaller values (and hence smaller grid sizes in s-direction) are necessary for larger angles. Note that this grid ratio is defined for the ML mirror's centre, and a static grid is used throughout the structure. Since the local angle of incidence may vary significantly, a local grid spacing might improve performance. But this introduces the need of interpolation. Further studies on the feasibility of a finite element method are wishful.

\section{D.5 Environment variables}

The Takagi-Taupin simulation programme for curved focusing ML mirrors obtains geometrical and physical parameters via POSIX environment variables. These can be set by a host programme (or simply your favourite shell); for convenience, a shell script setup. sh is available that exports default values. The variables and their meaning are summarised in table D.1. From the BASH shell, environment variables are set using the 
$\$$ export config_layers_number=50

$\$$./takagi-taupin

syntax; in $\mathrm{C} / \mathrm{C}++$, use the $\operatorname{setenv}(\ldots)$ function.

\section{D.6 Command line arguments}

In addition to the mandatory environment variables described in the previous section, optional parameters and flags may be set using command line arguments of the form

\$./takagi-taupin -acp --beta1=0 --beta2=0

Flags are denoted by single letters that can be combined, and are written behind a single hyphen-minus; options are written in full behind two hyphen-minus, their value is added behind an equality sign. Possible flags and options are shown in table D.2. They are parsed

\begin{tabular}{ll} 
Environment variable & Meaning \\
\hline config_geometry_s1 & $\begin{array}{l}\text { distance from source to mirror's centre } \\
\text { distance from there to focus }\end{array}$ \\
config_geometry_s2 & gracing angle of incidence at mirror's centre \\
config_illumination_center & $\begin{array}{l}\text { centre of illumination, inverse mirror length } \\
\text { spacing of multiple incoming beams, inverse mirror length } \\
\text { config_illumination_factor }\end{array}$ \\
config_illumination_file & (unused) \\
config_illumination_intens & intensity of illumination \\
config_illumination_method & (unused, should be self) \\
config_illumination_number & number of incoming beams (for testing) \\
config_illumination_shm & (unused) \\
config_illumination_width & width of incoming beam, inverse mirror length \\
config_layers_material1 & file name of tabulated optical index, first material \\
config_layers_material2 & file name of tabulated optical index, second material \\
config_layers_number & number of bilayers \\
config_layers_ratio & r-ratio of both layers \\
config_mirror_length & length of the ML mirror \\
config_physic_wavelength & vacuum-wavelength of illumination \\
config_shm_fieldout & key to shm segment for reflected amplitude \\
config_shm_plotdata & key to shm segment for intensity inside ML \\
config_simulation_gridpointss & number of discretisation points along the surface \\
config_simulation_gridpointst & number of discretisation points inside the structure \\
config_xocd_hostname & hostname of optical constants daemon \\
config_xocd_port & port number of optical constants daemon
\end{tabular}

Table D.1: List of environment variables and their meaning to set simulation parameters for Takagi-Taupin. 
using GNU's getopt and getopt_long functions ${ }^{\dagger}$. Flags can also be given with two hyphenminus signs and the full name listed in the table.

\footnotetext{
†http://www.gnu.org/s/hello/manual/libc/Getopt.html
}

\begin{tabular}{|c|c|}
\hline Flag/Option & Meaning \\
\hline$-a /--a s c i i$ & do not use unicode characters for output \\
\hline$-c /--\operatorname{colour}$ & use TERM escape sequences for colourful output \\
\hline$-d /--d r y$ & do (nearly) nothing, only print information \\
\hline$-f /--f l a t$ & simulate a flat $\mathrm{ML}$, illuminated with a plane wave \\
\hline -n/--nowrite & do not write to files or shm; useful for starting in parallel \\
\hline -p/--progressbar & enable progress bar during actual calculation \\
\hline -s/--smallfiles & $\begin{array}{l}\text { only create files of field amplitude at surface, not intensity within } \\
M L\end{array}$ \\
\hline - -factordelta $=\langle$ value $>$ & multiply optical constants of both materials \\
\hline - -factorbeta=<value $>$ & multiply optical constants of both materials \\
\hline$--\operatorname{delta1}=<$ value $>$ & overwrite optical constants of first material; real part \\
\hline-- delta $2=<$ value $>$ & overwrite optical constants of second material; real part \\
\hline - -beta1=<value $>$ & overwrite optical constants of first material; imaginary part \\
\hline- -beta $2=<$ value $>$ & overwrite optical constants of second material; imaginary part \\
\hline$--f i e l d-i n=<$ name $>$ & overwrite file name for incoming amplitude \\
\hline - field-out $=<$ name $>$ & overwrite file name for reflected amplitude \\
\hline$--f i e l d 0=<$ name $>$ & overwrite file name for incoming field inside the structure \\
\hline$--f i e l d 1=<$ name $>$ & overwrite file name for reflected field inside the structure \\
\hline-- maxmem $=\langle$ value $>$ & never use (much) more memory than given, in megabytes \\
\hline-- rot - thet $a=\langle v>$ & $\begin{array}{l}\text { use modification function of subsection D.3.1; value is rocking an- } \\
\text { gle in microradian }\end{array}$ \\
\hline- -rotation-theta $=<\mathrm{v}>$ & $\begin{array}{l}\text { use modification function of subsection D.3.2; value is rocking an- } \\
\text { gle in microradian }\end{array}$ \\
\hline - -modbragg-factor $=\langle\mathrm{v}\rangle$ & $\begin{array}{l}\text { use modification function of subsection D.3.3; value is modification } \\
\text { factor } f \text { in (4.18) }\end{array}$ \\
\hline
\end{tabular}

Table D.2: Flags and options for the Takagi-Taupin programme. Flags are basically used to change the output of the programme, whilst options change values and filenames. 
APPENDIX D. IMPLEMENTATION DETAILS OF TT 


\section{Appendix E}

\section{Command-line interface}

The $x$-ray multilayer mirror simulations (XMLS) offers a command-line based on the GNU readline library as a basic interface to the user. In addition, a web-based graphical user interface (GUI) has been implemented that is described in the following appendix. An example session of the command-line interface (CLI) has been given in appendix C.1; the commands and their parameter have been introduced in appendix C.2. The present appendix is devoted to the readline interface itself. The GNU readline library is known from a lot of Linux programmes, like bash, gnuplot, and others. It offers more than just a simple command line; in XMLS, history function and tabular completion assist the user.

History With the cursor keys "up" and "down", the user may recall previous commands, edit, and resubmit them to XMLS. Hitting the "page up" key searches the history for lines with the same start pattern as the currently typed input. Pressing "ctrl-r" allows for searching at arbitrary positions in the older lines.

Tabulator completion Pressing the "tabulator" key, readline tries to auto-complete the current start of a word; possible completions are the XMLS commands (as get, set etc.) at the start of a command, or the parameters (like mirror. length or physics. wavelength). If none matches, file names are completed. This is handy combined with ...

Shell escape The xmls command run means that the rest of the line shall be executed using the system (3) command that calls / bin/sh. This command proved useful for quickly navigating and manipulating the file system, calling external programmes such as the vim editor, or gnuplot. 
APPENDIX E. COMMAND-LINE INTERFACE 


\section{Appendix $F$}

\section{Web-based interface}

In addition to the GNU readline based command line interface (CLI), a web based graphical user interface (GUI) has been implemented in XMLS. Although control is a little slower, this allows for fast access to important plots.

The web GUI is served from the built-in HTTP server (see the following appendix for more details) as a set of HTML, CSS, and Javascript files that can easily be adapted. This approach seems more flexible than traditional GUI software. In fact, GUI and XMLS core are separate: simulations do continue if the client is no longer connected; the web GUI can be re-opened later from a different terminal, or multiple GUIs can control the same simulation.

A screenshot of XMLS' web-based GUI is reproduced in figures F.1 - F.3.

The simple layout is composed of three parts: a large plot on top, an overview of different plots below; on the right hand side, the current set of parameters is given. Clicking on a parameter asks the user for a new value; clicking "start" starts one simulation run (it is not yet possible to start loops from the GUI). From the overview of small plots, the top plot can be selected; often, the corresponding data file is linked.

There are several informative boxes placed between the "start" link and the parameter box. The current state of the simulation ("idle", "singlerun" etc.) is given in boldface; a progress counter (in percent) of the mirror simulation is placed next to it. If XMLS runs on a multi-user system, the output of the uptime(1) command found below is useful not to overload the system. The numbers estimate the average number of running jobs over the last $\{1,5,15\}$ minutes. 


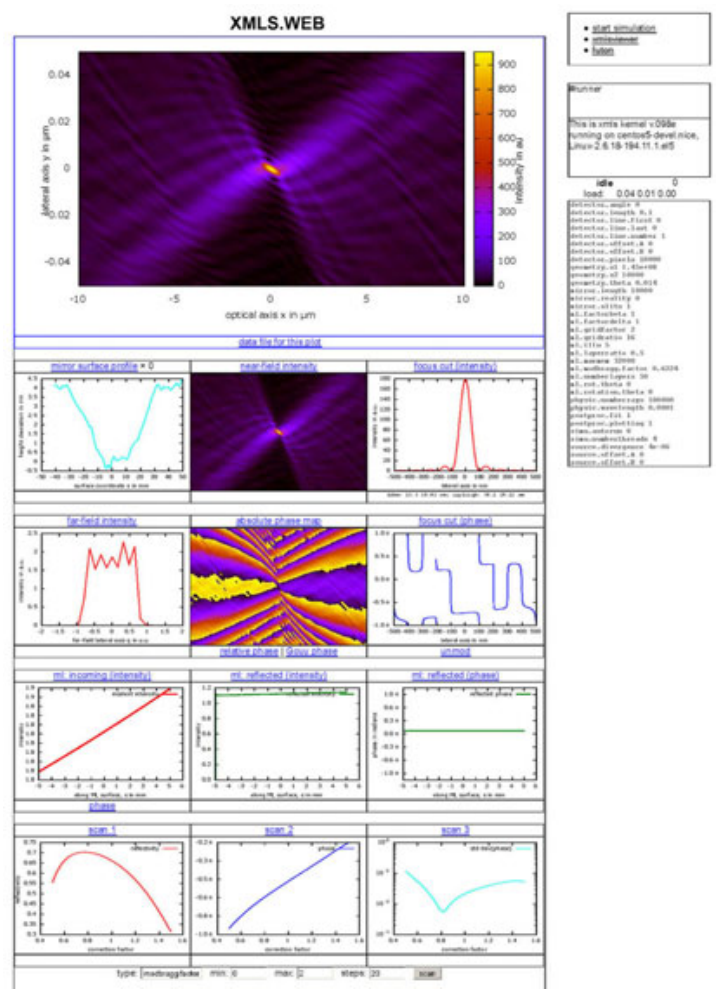

Figure F.1: Screenshot of XMLS' web GUI. A detailed reproduction is shown in figures F.2 - F.3.

(a)

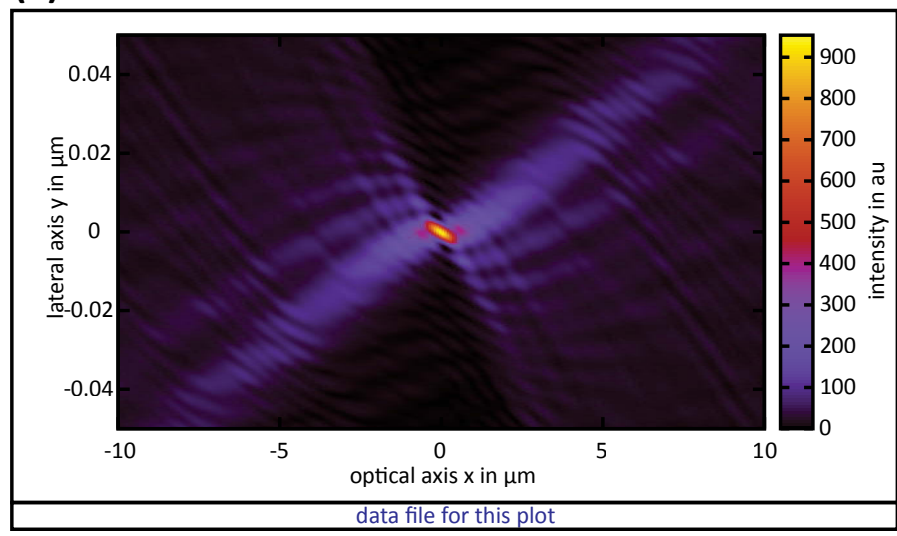

\begin{tabular}{|c|c|}
\hline \multicolumn{2}{|l|}{$\begin{array}{ll}\text { start simulation } & \text { (b) }\end{array}$} \\
\hline \multicolumn{2}{|l|}{ \#runner } \\
\hline \multicolumn{2}{|c|}{$\begin{array}{l}\text { This is xmls kernel v.098 running on } \\
\text { ruprecht, Linux-2.6.18-194.11.1.el5 }\end{array}$} \\
\hline $\begin{array}{ll}\text { idle } & 0 \\
\text { load: } 0.040 .010 .00 & \end{array}$ & \\
\hline $\begin{array}{l}\cdots \\
\text { geometry.s1 (C) } \\
\text { geometry.s2 } \\
\text { geometry.theta } \\
\text { mirror.length } \\
\ldots \\
\text { ml. numberlayers } \\
\ldots \text {. } \\
\text { physic.wavelength } \\
\ldots\end{array}$ & $\begin{array}{c}1.45 e+08 \\
10000 \\
0.014 \\
10000 \\
50 \\
0.0001\end{array}$ \\
\hline
\end{tabular}

Figure F.2: Detailed reproduction of XMLS' web GUI, continued in figure F.3. (a) enlarged plot, one selected from (aa) to (dc); here: intensity distribution in the focal region; (b) link to start a single simulation, with host information below; (c) list of all parameters; these are links to a Javascript query. 


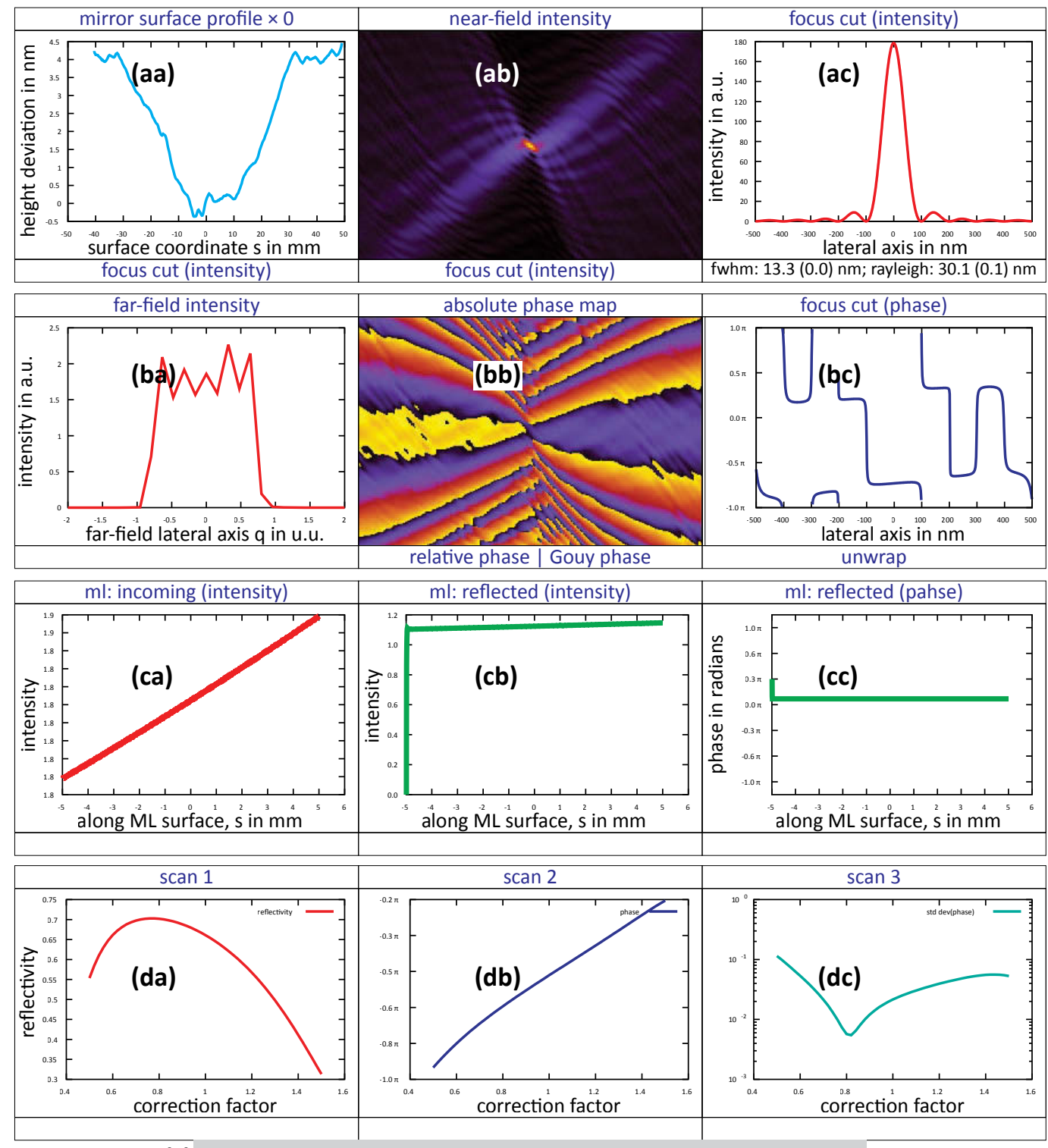

(s) type: modbragg.factor min: $0 \quad$ max: 2 steps: 20 scan

Figure F.3: Continuation of figure F.2: (aa) mirror height deviation profile; (ab) intensity distribution in the focal region; (ac) intensity cut in the focal plane; (ba) far-field obtained from a simple FFT (often very noisy); (bb) phase distribution in the focal region: "relative phase map" is with plane wave subtracted, "Gouy phase" is a cut along the optical axis; (bc) focus cut in the focal plane; (ca) incoming intensity on a ML mirror; (cb) reflected intensity along ML's surface; (cc) phase of the reflected wave along $M L$ 's surface; (da) reflectivity curve of a ML rocking scan; ( $d b$ ) reflected phase at ML's centre for a rocking scan; (dc) standard deviation of reflected phase, evaluated along ML's surface; (s) control form for rocking scan. 
APPENDIX F. WEB-BASED INTERFACE 


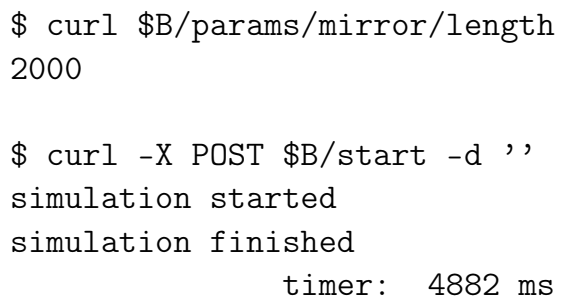

\$ curl \$B/data/intensity-cut.dat I head -3

$-0.1000004 .69926$

$-0.0980006 .81641$

$-0.0960009 .01234$

$\$$ curl $\$ B /$ fit/focus/fwhm 31.234

So the basic syntax is

\$ curl -X METHOD \$BASE/URL -d DATA,

where METHOD is either GET (the default, for retrieving information from XMLS) or POST (used for "writing", like changing a parameter or starting a new simulation), BASE is the base part of the address (containing the computer name and port number), URL is the path to the "information" (like a parameter, or start, or a data file name), and DATA may be the new value of a parameter (in case of start, it may be empty).

In the following sections, examples show how to remote control XMLS from other programmes like Gnuplot, MATLAB, or Mathematica. We also reproduce parts of a bash script that controls XMLS based on a file containing parameter sets.

\section{G.2 Obtaining data and plots}

Data files are accessible via the URL \$BASE/data/, where \$BASE is the base part of the URL, connecting to the XMLS programme. Possible data files are those listed in subsection C.4, like \$BASE/data/intensity-cut.dat or \$BASE/data/scanfile.dat. They can easily be accessed from within Gnuplot using curl:

gnuplot> plot '<curl \$BASE/data/scanfile.dat' with lines title 'scanfile'

\section{G.3 Example: MATLAB}

Remote control of XMLS via HTTP from Matlab is somewhat tricky, since Matlab's urlread function does not seem to allow for standard compliant POST. The following script uses a workaround using curl to set a new mirror length and start the simulation. 
01: base='http://centos5-devel :8080';

02: para='params/mirror/length';

03:

04: url=sprintf $\left(\% \mathrm{~s} / \% \mathrm{~s}^{\prime}\right.$, base, para $)$;

05: urlread (url)

06:

07: length=80e3;

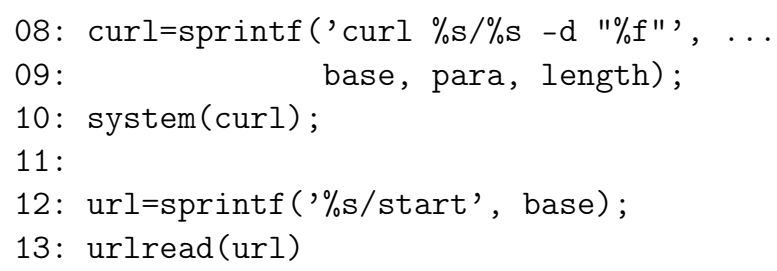

\section{G.4 Example: Mathematica}

In figure G.1 we give a short example how to start a simulation and plot the result from within Mathematica.

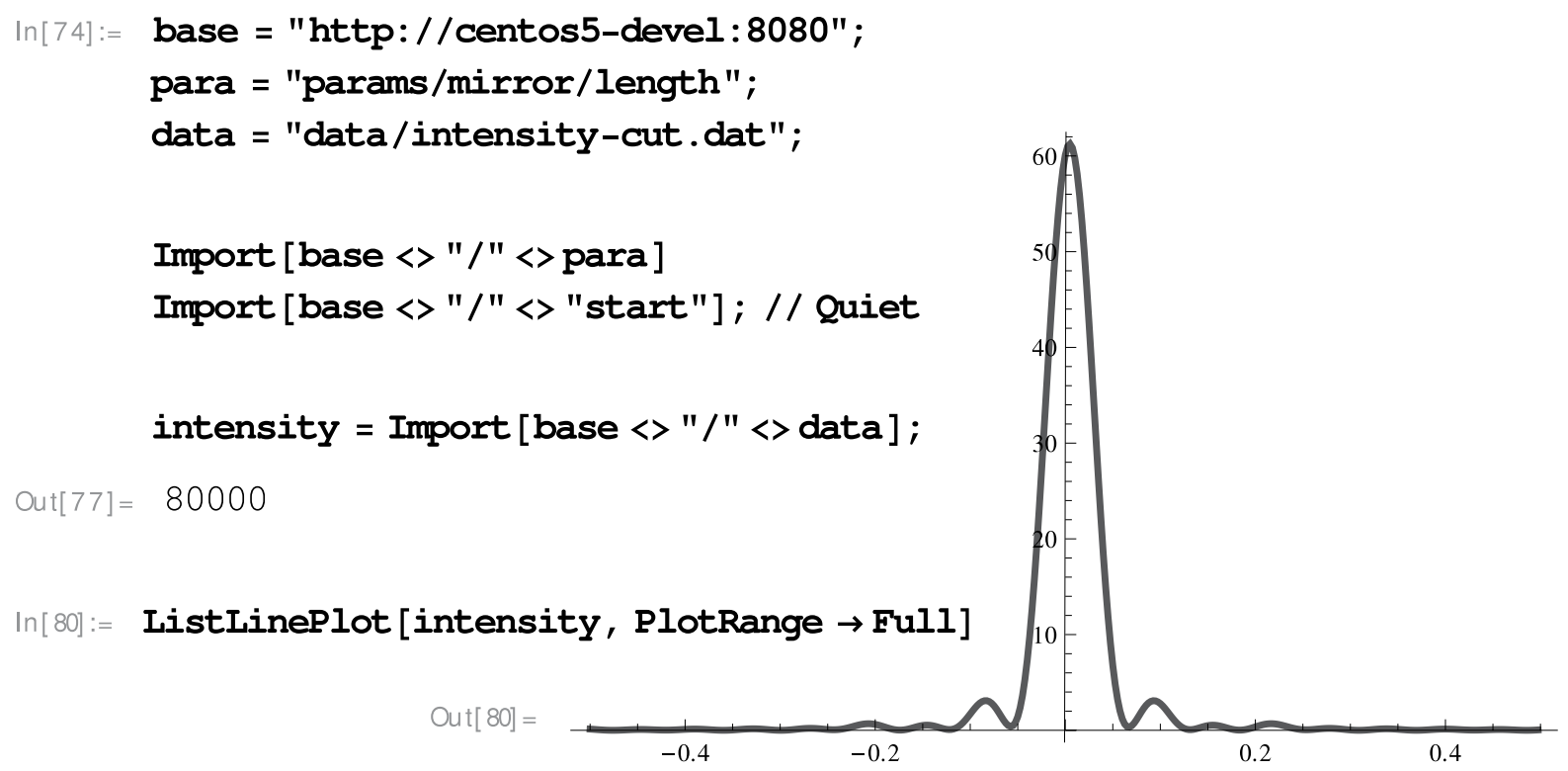

Figure G.1: Reproduction of a Mathematica notebook showing how to start an XMLS simulation, load the result, and plot it.

\section{G.5 Example: bash script}

In this section we reproduce parts of a bash script used to automate the calculations for figure 4.23. Given an input file containing parameter sets as shown below, this script controls XMLS and aggregates the results. 
The script scan.sh:

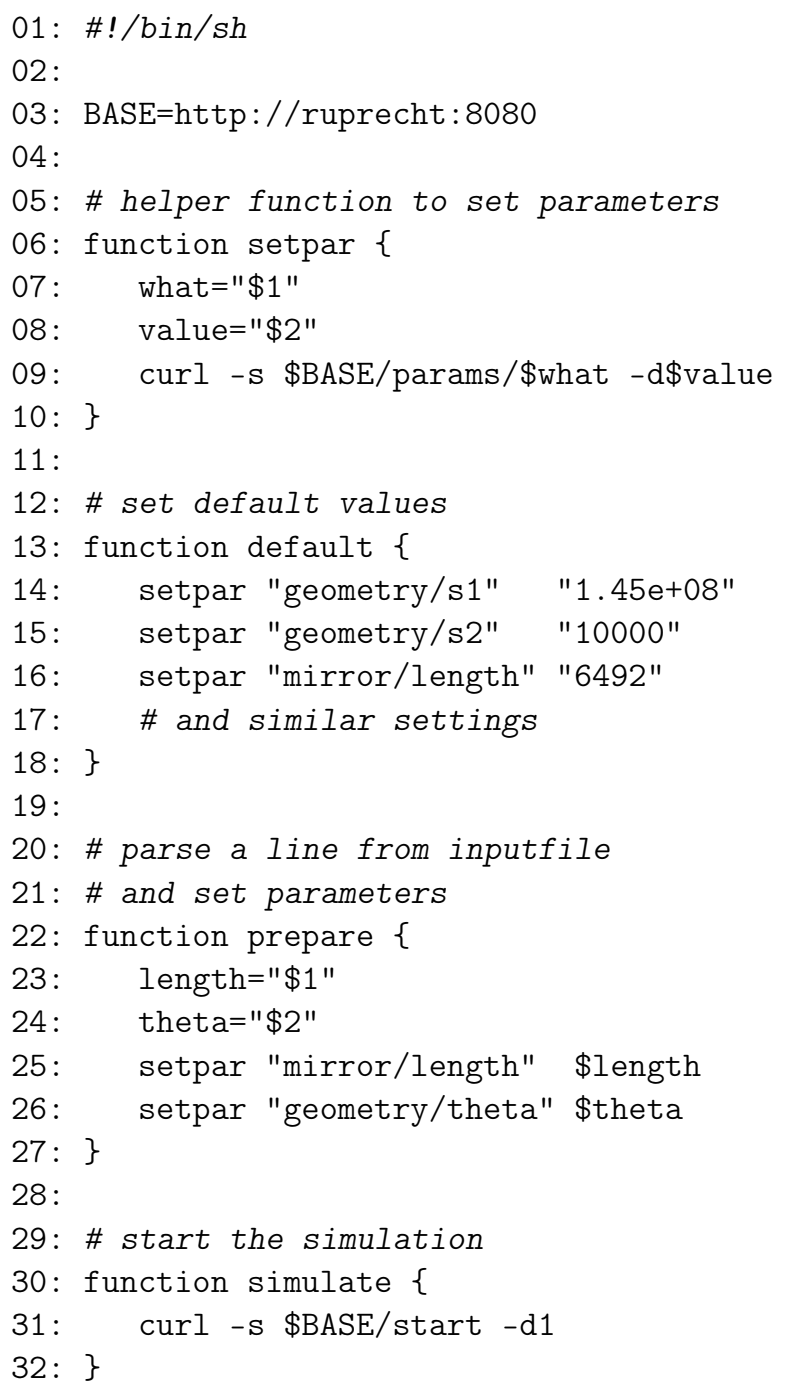

The input and output files:

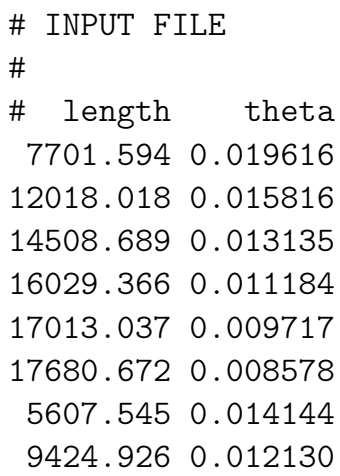

33:

34: \# obtain focus sizes and data files

35: function getdata \{

36: fwhm='curl -s \$BASE/fit/focus/fwhm'

37: file $=$ 'date $+\% \mathrm{~s}^{\text {' }}$

38: echo -e "\t\$@ \$fwhm \$file"

39: curl \$BASE/data/ml_reflected.dat >

40: reflected/\$file.dat

41: curl \$BASE/data/intensity.dat >

42: $\quad$ intensities/\$file.dat

43: $\}$

44:

45: \# set default parameters

46: default

47: \# clear outputfile

48: : >outputfile

49:

50: \# read inputfile

51: while read line

52: do

53: first $=\$\{$ line: $0: 1\}$

54: [ \$first $=$ "\#" ] \&\& continue

55: echo -e "\t\$line"

$56:$

57: prepare \$line

58: simulate

59: getdata $\$$ line | tee -a outputfile

60:

61: done < inputfile

62: echo | tee -a outputfile 63:

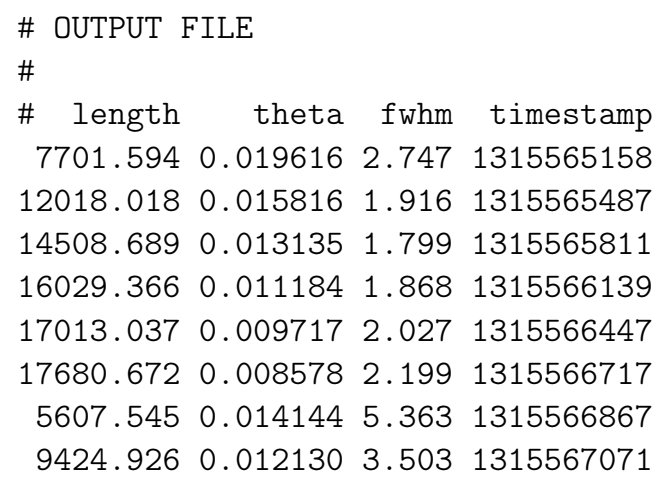




\section{Appendix $\mathrm{H}$}

\section{Modified Bragg condition}

In subsection 4.3.2, an approximative expression of the deviation $\varepsilon \approx-4 \delta$ has been derived from a model function $\varphi(s, t)$ to describe $M L$ mirrors constructed following the modified Bragg condition (4.13). Using the Mathematica software by Wolfram Research, version 8.0, we obtain numerical values of $\varepsilon(s, t)$ that are in good agreement to this approximation.

Figure H.1 shows the Mathematica source code used to produce the plots in H.2. In the left column, first some functions are defined, see the derivations of chapter 4 . Then the Bragg layer spacing $\Lambda 1$ and the modified version $\Lambda 2$ are defined, and a "stretching" of the $t$-coordinate is constructed from their ratio. The deviation $\varepsilon$ is given as a sum of derivatives, see subsection 4.2.2. The code in the right column are plot commands and define the geometrical parameters, as summarised in table H.1. The plots are two line cuts $\varepsilon(s)$ (figure H.2 (a)) and $\varepsilon(t)$ (figure H.2 (b)); in (b), the cut is at the mirror's centre, $s=s_{0}$; for (a), $t$ has been put to three values: $t-t_{0}=\{0.0,0.5,1.0\} \mu \mathrm{m}$.

According to figure H.2 (a), the deviation function $\varepsilon(s, t) \approx-4 \delta$, as derived in subsection 4.3.2. At small angles (on the "left" side of the ML mirror), actual values are larger (in absolute value) by about two per cent in this model case. Also a small gradient in $t$-direction is visible; this can be better seen in the cut $\varepsilon\left(s=s_{0}, t\right)$ of figure H.2 (b). Estimating the derivatives of $\varphi(s, t)$, at first it seems that $\partial_{t} \varphi \gg \partial_{s} \varphi$. But usual ML mirrors are rather thin $-T \lesssim 1 \mu \mathrm{m}-$ and long $-S \sim 10 \mathrm{~mm}$, so $\varepsilon$ changes more with $s$ than with $t$. 


\begin{aligned} Quantity & Value \\ \hline distance from source & $50.0 \mathrm{~m} \\$ distance to focus & $0.1 \mathrm{~m} \\$ length of mirror & $16.0 \mathrm{~mm} \\$ angle at mirror's centre & $10.0 \mathrm{mrad} \\$ average index of refraction & $n=1-1 \times 10^{-6}\end{aligned}$

Table H.1: Geometrical parameters for figure H.2.

$$
\begin{aligned}
& \alpha\left[s_{-}, t_{-}\right]:=\frac{c^{2}-s^{2}}{t^{2}-s^{2}} ; \quad \quad \alpha^{2}(s, t) \\
& \beta\left[s_{-}, t_{-}\right]:=\frac{t^{2}-c^{2}}{t^{2}-s^{2}} ; \quad B^{2}(s, t) \\
& \theta\left[s_{-}, t_{-}\right]=\sqrt{\beta[s, t]} ; \quad \text { local angle } \vartheta(s, t) \\
& \Lambda 1=\frac{\lambda}{2 \operatorname{Sin}[\theta[s, t]]} ; \quad \text { Bragg's law } \\
& \Lambda 2=\frac{\lambda}{2 \sqrt{(1-\delta)^{2}-\operatorname{Cos}[\theta[s, t]]^{2}}} ; \quad \begin{array}{l}
\text { modified Bragg } \\
\text { condition }
\end{array} \\
& \text { phi }\left[s_{-}, t_{-}\right]:=(t-t 0)\left(\frac{\Lambda 2}{\Lambda 1}-1\right) / . \lambda \rightarrow \begin{array}{l}
10^{-10} ; \\
\text { stretching factor }
\end{array} \\
& \alpha[s, t]\left(+2 D[p h i[s, t], s]+D[p h i[s, t], s]^{2}\right) \text {; } \\
& \beta[s, t]\left(-2 D[p h i[s, t], t]+D[p h i[s, t], t]^{2}\right) \text {; } \\
& \text { fun }=2(\%+\%) \text {; } \\
& \text { deviation function, } \\
& \text { see subsection 4.2.2 }
\end{aligned}
$$

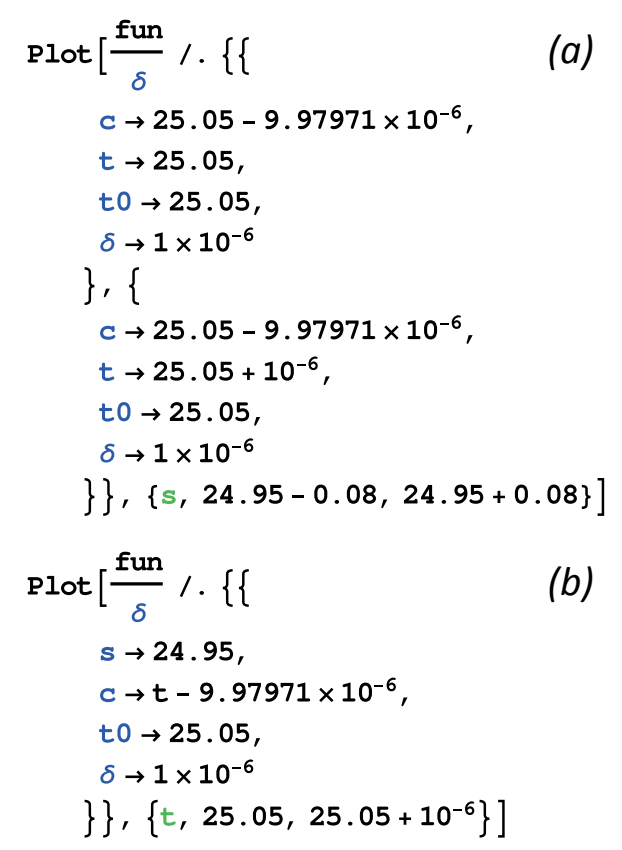

Figure H.1: Mathematica notebook to produce plots (a) and (b) in the following figure.

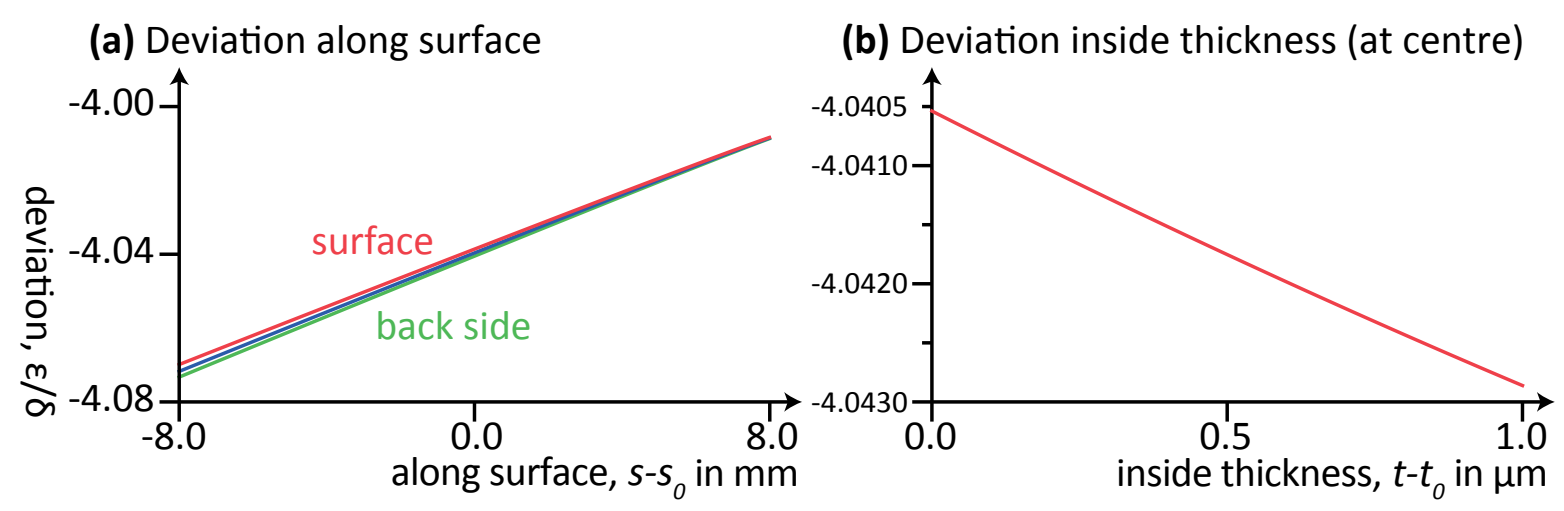

Figure H.2: Deviation function $\varepsilon(s, t)$, along surface $s(a)$ and along thickness $t(b)$. The value is approximately $-4 \delta$, as derived in (4.15). 


\section{Appendix I}

\section{X-ray optical constants daemon}

The simulation programmes written and used for this thesis rely on optical constants that describe matter's interaction with $\mathrm{x}$-ray photons. Often it is appropriate to assume homogeneous media described by a complex valued index of refraction, $n=1-\delta+i \beta$. The coefficients $\delta$ and $\beta$ depend on material and photon energy. For a large variety of elements and compounds, and for large energy ranges, these values have been determined experimentally and tabulated. For this thesis, the database of the IMD software suite, written by David Windt, has been used [52]. Interpolation of data in between measured values has been done linearly.

A stand-alone web server XOCD ( $\mathrm{x}$-ray optical constants daemon) has been programmed as a wrapper to access IMD's files from remote, including linear interpolation. For convenience, the core part has also been included in the XMLS programme.

From the index of refraction $n$ the susceptibility $\chi$ is given as

$$
\chi=n^{2}-1 .
$$

Multilayers considered in this work consist of two materials, described by their indexes of refraction $n_{1}$ and $n_{2}$; the layer thickness ratio is denoted by $\Gamma$. Assuming now a square wave with wave number $\tilde{h}$, the Fourier series expansion in first order is given as

$$
\chi(t)=\Gamma \chi_{\text {mat } 1}+(1-\Gamma) \chi_{\text {mat } 2}+\frac{2}{\pi}\left(\chi_{\text {mat } 1}-\chi_{\text {mat } 2}\right) \sin (\tilde{h} t)-\frac{2}{\pi}\left(\chi_{\text {mat } 1}-\chi_{\text {mat } 2}\right) \sin (-\tilde{h} t),
$$

see figure I.1. In first order, layer ratio $\Gamma$ only contributes to average susceptibility $\chi_{0}$ and does not influence Fourier components $\chi_{ \pm 1}$. In this form we assume that the first layer is described by $\chi_{\text {mat }}$. Numerical values of the coefficients for different photon energies and a $\mathrm{W} / \mathrm{B}_{4} \mathrm{C}$-multilayer are given in table I.1. 


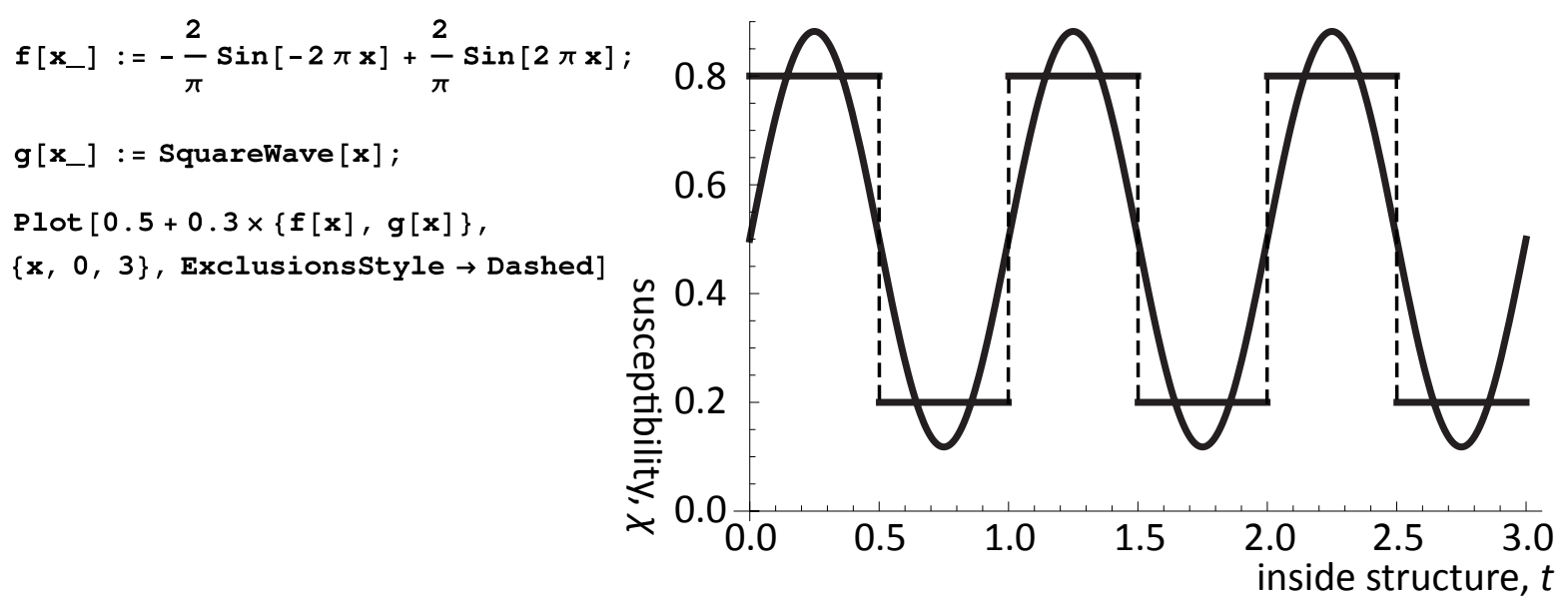

Figure I.1: First order Fourier representation of a square wave by a superposition of two sine waves with wave numbers $\pm \tilde{h}$.

\begin{tabular}{rll} 
Photon energy & index of refraction, $W$ & index of refraction, $\mathrm{B}_{4} \mathrm{C}$ \\
\hline $12.4 \mathrm{keV}$ & $1-1.951 \times 10^{-5}+3.305 \times 10^{-6} i$ & $1-3.224 \times 10^{-6}+1.187 \times 10^{-9} i$ \\
$17.5 \mathrm{keV}$ & $1-1.045 \times 10^{-5}+9.601 \times 10^{-7} i$ & $1-1.609 \times 10^{-6}+2.643 \times 10^{-0} i$ \\
$24.8 \mathrm{keV}$ & $1-5.228 \times 10^{-6}+2.668 \times 10^{-7} i$ & $1-7.749 \times 10^{-7}+5.755 \times 10^{-1} i$ \\
$49.6 \mathrm{keV}$ & $1-1.273 \times 10^{-6}+2.072 \times 10^{-8} ;$ & $1-1.788 \times 10^{-7}+3.098 \times 10^{-2} i$
\end{tabular}

\begin{tabular}{rll} 
Photon energy & $\chi_{0}$ & $\chi_{1}$ \\
\hline $12.4 \mathrm{keV}$ & $-2.273 \times 10^{-5}+3.306 \times 10^{-6} i$ & $-2.074 \times 10^{-5}+4.206 \times 10^{-6} i$ \\
$17.5 \mathrm{keV}$ & $-1.206 \times 10^{-5}+9.604 \times 10^{-7} i$ & $-1.126 \times 10^{-5}+1.222 \times 10^{-6} i$ \\
$24.8 \mathrm{keV}$ & $-6.003 \times 10^{-6}+2.669 \times 10^{-7} i$ & $-5.670 \times 10^{-6}+3.396 \times 10^{-7} i$ \\
$49.6 \mathrm{keV}$ & $-1.452 \times 10^{-6}+2.072 \times 10^{-8} i$ & $-1.393 \times 10^{-6}+2.638 \times 10^{-8} i$
\end{tabular}

Table I.1: Index of refraction and Fourier coefficients of the susceptibility for $W / B_{4} C$ multilayers at different photon energies, as used within this thesis. Values for $n$ are taken from the IMD database [52]. Not given are $\chi_{\overline{1}}=-\chi_{1}$. 


\section{Appendix J}

\section{Comparison: GCC 4.3 vs. 4.1}

In appendix $C$ it is pointed out that the GNU compiler collection, version 4.3, produces faster code for the Fresnel-Kirchhoff integral used in the class CPDLine that GCC 4.1. This integral needs to calculate phase factors $\exp (i k x)$; in the $\mathrm{C}++$-code, this complex factor is rewritten using Euler's identity, so the sine and cosine are calculated; before, the remainder of $k x$ with $2 \pi$ is obtained using fmod. On the following page, fragments of assembler code generated by GCC 4.1.2 (Red Hat 4.1.2-48) (left column) and GCC 4.3.4 (built from source) (right column), unoptimised, for a 64 bit Intel Xeon CPU (X5472, running at $3 \mathrm{GHz}$ ), are compared. Differing lines have are indicated by a boldface line number. The translated C-code function is printed on top. Timing results of $100 \times 10^{6}$ evaluations with different optimisation flags are shown in table J.1.

The two compiler versions generate very different (unoptimised) code. GCC 4.3 uses Intel's FPREM (floating point, partial remainder) instruction to calculate the remainder of ST (0)/ST (1) by iterative subtraction. This remainder differs from IEEE 754. The exponent of ST (0) is reduced by no more than 63 in one execution, which is the reason for the loop with address .L9. With optimisation enabled, GCC 4.3 improves the loop around FPREM with a tremendous speed-up.

\begin{tabular}{rrl} 
Optimisation & GCC 4.1 & GCC 4.3 \\
\hline$-\mathrm{O} 0$ & $12.835(32)$ & $6.748(67)$ \\
$-\mathrm{O} 1$ & $7.787(12)$ & $0.809(2)$ \\
$-\mathrm{O} 2$ & $7.862(9)$ & $0.777(1)$ \\
$-\mathrm{O} 3$ & $7.861(11)$ & $0.781(15)$
\end{tabular}

Table J.1: Run-time comparison for $10^{8}$ evaluations of the doit-function, compiled with GCC 4.1 and GCC 4.3, with indicated optimisation flags. Times are in seconds. Error estimations are standard deviation of twenty consecutive runs. 
C-code: double doit(int $i$, int $j)\left\{\operatorname{return} \sin \left(\operatorname{fmod}\left(i+j, 2 * M_{-} P I\right)\right) ;\right\}$

\begin{tabular}{|c|c|c|}
\hline $\begin{array}{r}\text { Assembler } \\
\text { GCC } 4 .\end{array}$ & ode (gcc & $-\mathrm{S}-00)$ \\
\hline 01: doit: & pushq & $\%$ rbp \\
\hline 02: & movq & $\%$ rsp, $\%$ rbp \\
\hline 03: & subq & $\$ 16, \%$ rsp \\
\hline 04: & movl & $\%$ edi, $-4(\%$ rbp $)$ \\
\hline 05: & movl & $\%$ esi, $-8(\%$ rbp $)$ \\
\hline 06: & movl & $-4(\%$ rbp $), \%$ eax \\
\hline $07:$ & addl & $-8(\% \mathrm{rbp}), \%$ eax \\
\hline 08 : & & \\
\hline 09 : & cvtsi2sd & d $\quad \%$ eax,$\% x m m 0$ \\
\hline 10: & movabsq & $\$ 4618760256179416344, \% \operatorname{rax}$ \\
\hline 11: & movq & $\% \operatorname{rax}, \quad-16(\%$ rbp $)$ \\
\hline 12: & movsd & $-16(\%$ rbp $), \% x m m 1$ \\
\hline
\end{tabular}

GCC 4.3

$\begin{array}{ll}\text { doit: } & \text { pushq } \% \text { rbp } \\ \text { movq } & \% \text { rsp, } \% \text { rbp } \\ \text { subq } & \$ 64, \% \text { rsp } \\ \text { movl } & \% \text { edi, }-4(\% \text { rbp }) \\ \text { movl } & \% \text { esi },-8(\% \text { rbp }) \\ \text { movl } & -4(\% \text { rbp }), \% \text { eax } \\ \text { movl } & -8(\% \text { rbp }), \% \text { edx } \\ \text { leal } & (\% r d x, \% \text { rax }), \% \text { eax }\end{array}$

cvtsi2sd $\%$ eax, $\% x m m 0$

movsd $\% x m m 0,-24(\%$ rbp $)$

fldl .LC5 (\% rip)

fstpt $-64(\%$ rbp $)$

fldl $-24(\%$ rbp $)$

fstpt $-48(\%$ rbp $)$

.L9: fldt $-48(\% \mathrm{rbp})$

fldt $\quad-64(\%$ rbp $)$

fxch $\%$ st (1)

fprem

fxch $\%$ st (1)

fstpt $-64(\%$ rbp $)$

fstpt $-48(\% \mathrm{rbp})$

fnstsw $\%$ ax

testb $\$ 4, \%$ ah

jne .L9

fldt $\quad-48(\%$ rbp $)$

fstpl $-32(\%$ rbp $)$

movsd $-32(\% \mathrm{rbp}), \% \mathrm{xmm0}$

ucomisd $-32(\%$ rbp $), \% x m m 0$

jp .L12

je $\quad$.L10

L12: movsd .LC5( $\%$ rip), \%xmm0

movapd $\% x m m 0, \% x m m 1$

movsd $-24(\% \mathrm{rbp}), \% \mathrm{xmm0}$

call fmod

movsd $\% x m m 0,-32(\%$ rbp $)$

.L10: movsd $-32(\% \mathrm{rbp}), \% \mathrm{xmm0}$

call sin leave

ret 


\section{Bibliography}

[1] Wilhelm Conrad Röntgen. Über eine neue Art von Strahlen. Sitzungsberichte der physikal.-medizin. Gesellschaft, page 123, 1895.

[2] Walter Friedrich, Paul Knipping, and Max von Laue. Interferenz-Erscheinungen bei Röntgenstrahlen. Sitzungsberichte der Mathematisch-Physikalischen Classe der Königlich-Bayerischen Akademie der Wissenschaften zu München, 303, 1912.

[3] Max Born. Optik. Springer-Verlag, Berlin, Heidelberg, 1965.

[4] Wolfgang Demtröder. Elektrizität und Optik (Experimentalphysik, Bd.2). Springer, Berlin, 2002.

[5] Dieter Meschede, editor. Gerthsen Physik. Springer, Berlin, 2010.

[6] Leonard Mandel and Emil Wolf. Optical Coherence and Quantum Optics. Cambridge University Press, Cambridge, 1995.

[7] Emil Wolf. Introduction to the theory of coherence and polarization of light. Cambridge University Press, Cambridge, 2007.

[8] Jens Als-Nielsen and D. McMorrow. Elements of Modern X-ray Physics. John Wiley \& Sons, Chichester, 2011.

[9] Gianluca Geloni, E. Saldin, L. Samoylova, E. Schneidmiller, H. Sinn, Th. Tschentscher, and M. Yurkov. Coherence properties of the European XFEL. New Journal of Physics, 12(3):035021, 2010.

[10] K. Balewski, W. Brefeld, W. Design, H. Franz, R. Röhlsberger, and Edgar Weckert. PETRA III: A Low Emittance Synchrotron Radiation Source. Technical Design Report. DESY, Hamburg, 2004.

[11] Ivan A. Vartanyants and Andrei Singer. Coherence properties of hard x-ray synchrotron sources and x-ray free-electron lasers. New Journal of Physics, 12(3):035004, 2010.

[12] Tim Salditt, Sebastian Kalbfleisch, Markus Osterhoff, Sven Phillip Krüger, Matthias Bartels, Klaus Giewekemeyer, Henrike Neubauer, and Michael Sprung. Partially coherent nano-focused x-ray radiation characterized by Talbot interferometry. Optics Express, 19(10):9656, 2011. 
[13] Raphael Klünder, Fabia Masiello, Pierre van Vaerenbergh, and Jürgen Härtwig. Measurement of the spatial coherence of synchrotron beams using the Talbot effect. Phys. Status Solidi A, 206:1842, 2009.

[14] Alexander Rack, Timm Weitkamp, T. Rack, A. Cecilia, P. Vagovič, E. Harmann, Rainer Dietsch, and $\mathrm{H}$. Riesemeier. Coherence preservation and beam flatness of a single-bounce multilayer monochromator (beamline ID19- ESRF). Nuclear Instruments and Methods in Physics Research Section A, 2011.

[15] Jean-Pierre Guigay, Simon Zabler, Peter Cloetens, Christian David, Rajmund Mokso, and Michel Schlenker. The partial Talbot effect and its use in measuring the coherence of synchrotron X-rays. J. Synchrotron Radiation, 11(6):476-482, 2004.

[16] Henry Fox Talbot. Facts relating to optical science. Philos. Mag., 9:401, 1836.

[17] Emil Wolf and Taco D. Visser. The origin of the Gouy phase anomaly and its generalization to astigmatic wavefields. Optics Communications, 283:3371-3375, 2010.

[18] Mark Fox. Quantum Optics: An introduction. Oxford University Press, Oxford, 2006.

[19] Trina Bouvet, Carsten Detlefs, Edward Mitchell, and Jean-Luc Revol, editors. The Science and Technology Programme 2008-2017. ESRF, Grenoble, 2007.

[20] K. Yamauchi, K. Yamamura, H. Mimura, Y. Sano, A. Saito, A. Souvorov, M. Yabashi, K. Tamasaku, T. Ishikawa, and Y. Mori. Nearly diffraction-limited line focusing of a hard-x-ray beam with an elliptically figured mirror. Journal of Synchrotron Radiation, 9:313-316, 2002.

[21] Tetsuya Ishikawa, Kenji Tamasaku, Makina Yabashi, Shunji Goto, Y. Tanaka, Hiroshi Yamazaki, Kunikazu T. Takeshita, Hidekazu Kimura, H. Ohashi, T. Matsushita, and T. Ohata. 1-km beamline at SPring-8. SPIE proceedings, 4145, 2001.

[22] A. C. Schell. Multiple Plate Antenna. PhD thesis, Massachusetts Institute of Technology, 1961.

[23] Eugene Hecht. Optik. Oldenbourg, München, 2001.

[24] P. H. van Cittert. Die wahrscheinliche Schwingungsverteilung in einer von einer Lichtquelle direkt oder mittels einer Linse beleuchteten Ebene. Physica, 1:201-210, 1934.

[25] F. Zernike. The concept of degree of coherence and its application to optical problems. Physica, 5(8):785-795, 1938.

[26] E. Tervonen, Jari Turunen, and Ari T. Friberg. Transverse laser-mode structure determination from spatial coherence measurements: Experimental results. Applied Physics B: Lasers and Optics, 49(5):409-414, 1989.

[27] J. V. Cornacchio and K. A. Farnham. On the measurement of the complex spatial coherence of a He-Ne laser beam. II Nuovo Cimento B, 42(1):108-120, 1966.

[28] Joseph W. Goodman. Statistical Optics. John Wiley \& Sons, Chichester, 2000. 
[29] Sven Philip Krüger, Klaus Giewekemeyer, Sebastian Kalbfleisch, Matthias Bartels, Henrike Neubauer, and Tim Salditt. Sub-15 nm beam confinement by two crossed $x$-ray waveguides. Optics Express, 18(13):13492-13501, 2010.

[30] Klaus Giewekemeyer, Henrike Neubauer, Sebastian Kalbfleisch, Sven Philip Krüger, and Tim Salditt. Holographic and diffractive $x$-ray imaging using waveguides as quasi-point sources. New Journal of Physics, 12(3):035008, 2010.

[31] Markus Osterhoff and Tim Salditt. Real structure effects in x-ray waveguide optics: The influence of interfacial roughness and refractive index profile on the near-field and far-field distribution. Optics Communications, 282(16):3250-3256, 2009.

[32] Anika Kohlstedt, Sebastian Kalbfleisch, Tim Salditt, M. Reiche, U. Gösele, E. Lima, and P. Willmott. Two-dimensional $x$-ray waveguides: fabrication by wafer-bonding process and characterization. Applied Physics A, 91:7-12, 2008.

[33] Christian Fuhse, Christoph Ollinger, and Tim Salditt. Waveguide-based off-axis holography with hard x-rays. Physical Review Letters, 97(25):254801, 2006.

[34] Inna Bukreeva, Alexander Popov, Daniele Pelliccia, Alessia Cedola, S. B. Dabagov, and Stefano Lagomarsino. Wave-field formation in a hollow x-ray waveguide. Physical Review Letters, 97(18):184801, 2006.

[35] Franz Pfeiffer, Christian David, Manfred Burghammer, Christian Riekel, and Tim Salditt. Two-dimensional x-ray waveguides and point sources. Science, 297:230, 2002.

[36] Sebastian Kalbfleisch, PhD thesis. Georg-August-Universität Göttingen, in preparation.

[37] Henrike Neubauer, PhD thesis. Georg-August-Universität Göttingen, in preparation.

[38] Markus Osterhoff. Numerische Modellierungen von Röntgenwellenleitern unter Berücksichtigung von Realstruktureffekten. Diplomarbeit, Georg-August-Universität Göttingen, 2008.

[39] Anatoly Snigirev, Victor Kohn, Irina Snigireva, and Bruno Lengeler. A compound refractive lens for focusing high-energy x-rays. Nature, 384(6604):49-51, 1996.

[40] Christian G. Schroer and Bruno Lengeler. Focusing hard x-rays to nanometer dimensions by adiabatically focusing lenses. Phys. Rev. Lett., 94(5):054802, 2005.

[41] Anatoly Snigirev, Irina Snigireva, Gavin Vaughan, Jonathan Wright, Michel Rossat, A. Bytchkov, and Caroline Curfs. High energy x-ray transfocator based on Al parabolic refractive lenses for focusing and collimation. J. Phys.: Conf. Ser., 186:012073, 2009.

[42] Bastian Niemann, Dietbert Rudolph, and Günter Schmahl. Soft x-ray imaging zone plates with large zone numbers for microscopic and spectroscopic applications. Optics Communications, 12(160), 1974.

[43] Hanfei Yan, Jörg Maser, Albert T. Macrander, Qun Shen, Stefan Vogt, G. Brian Stephenson, and Hyon Chol Kang. Takagi-Taupin description of X-ray dynamical diffraction from diffractive optics with large numerical aperture. Physical Review B, 76:115438, 2007. 
[44] Tobias Liese, Volker Radisch, Inga Knorr, Michael Reese, Peter Großmann, Klaus Mann, and Hans-Ulrich Krebs. Development of laser deposited multilayer zone plate structures for soft x-ray radiation. Applied Surface Science, 257(12):5138-5141, 2011.

[45] Aike Ruhlandt, Tobias Liese, Sven Philip Krüger, Markus Osterhoff, Klaus Giewekemeyer, Hans Ulrich Krebs, and Tim Salditt. Sub-10nm x-ray focusing with a combined KB mirror and multilayer Laue lens. in preparation, 2011.

[46] Christian Morawe and Markus Osterhoff. Hard x-ray focusing with curved reflective multilayers. X-Ray Optics and Instrumentation, 2010:479631, 2010.

[47] Christian Morawe and Markus Osterhoff. Curved graded multilayers for x-ray nano-focusing optics. Nuclear Instruments and Methods in Physics Research Section A, 616:98-104, 2009.

[48] Paul Kirkpatrick and Albert Vincio Baez. Formation of optical images by x-rays. J. Opt. Soc. Am, 38:766-773, 1948

[49] K. Yamamura, H. Mimura, K. Yamauchi, Y. Sano, A. Saito, T. Kinoshita, K. Endo, Y. Mori, A. Souvorov, M. Yabashi, K. Tamasaku, and T. Ishikawa. Aspheric surface fabrication in nm-level accuracy by numerically controlled plasma chemical vaporization machining (CVM) and elastic emission machining (EEM). SPIE proceedings, 4782:265-270, 2002.

[50] Cameron M. Kewish, Lahsen Assoufid, Albert T. Macrander, and Jun Qian. Wave-optical simulation of hard-x-ray nanofocusing by precisely figured elliptical mirrors. Applied Optics, 46(11):2010-2021, 2007.

[51] Fritz Goos and Hilda von Hänchen. Ein neuer und fundamentaler Versuch zur Totalreflexion. Annalen der Physik, 436:333-346, 1947.

[52] David L. Windt. IMD - Software for modeling the optical properties of multilayer films. Computers in Physics, 12(4):360-370, 1998.

[53] Sebastian Kalbfleisch, Markus Osterhoff, Klaus Giewekemeyer, Henrike Neubauer, Sven Philip Krüger, Bastian Hartmann, Matthias Bartels, Michael Sprung, Olaf Leupold, Frank Siewert, and Tim Salditt. The holography endstation of beamline P10 at PETRA III. SRI 2009, 10th International Conference on Radiation Instrumentation, 1234(1):433-436, 2010.

[54] Max Born and Emil Wolf. Principles of optics: 7th (expanded) edition. Cambridge University Press, Cambridge, 1999.

[55] M.J. Bedzyk, D.H. Bilderback, G.M. Bommarito, M. Caffrey, and J.S. Schildkraut. X-ray standing waves: A molecular yardstick for biological membranes. Science, 241:1788, 1988.

[56] Fritz Goos and Hilda von Hänchen. Über das Eindringen des totalreflektierten Lichtes in das dünnere Medium. Annalen der Physik, 435:383-392, 1943.

[57] Gustav Robert Kirchhoff. Zur Theorie der Lichtstrahlen. Annalen der Physik, 2(18):663, 1883. 
[58] Arnold Sommerfeld. Mathematische Theorie der Diffraction. Mathematische Annalen, 21, 1895.

[59] P. Varga and Peter Török. Exact and approximate solutions of Maxwell's equations for a confocal cavity. Optics Letters, 21(19):1523, 1996.

[60] Donald Erwin Knuth. The Art of Computer Programming, Volume 2, 3rd edition. Addison-Wesley, Boston, 1997.

[61] Markus Osterhoff and Tim Salditt. Coherence filtering of $x$-ray waveguides: analytical and numerical approach. New Journal of Physics, 13(10):103026, 2011.

[62] Frank Siewert, Tino Noll, Thomas Schlegel, Thomas Zeschke, and Heiner Lammert. The nanometer optical component measuring machine: a new sub-nm topography measuring device for x-ray optics at BESSY. AIP Conf. Proc., 705:847-850, 2004.

[63] Emil Wolf and Edward Hubert Linfoort. Phase distribution near focus in an aberration-free diffraction image. Proc. Phys. Soc. B, 69(8):823, 1956.

[64] Fritz Zernike and Bernard Nijboer. Théorie des Images Optiques. Éditions de la Revue d'Optique, Paris, 1949.

[65] Eugen von Lommel. Abh. Bayer Akad. Wiss., 53:233, 1885.

[66] Cameron M. Kewish, Pierre Thibault, Martin Dierolf, Oliver Bunk, A. Menzel, J. Vila-Comamala, K. Jefimovs, and Franz Pfeiffer. Ptychographic characterization of the wavefield in the focus of reflective hard x-ray optics. Ultramicroscopy, 110(4):325-320, 2010.

[67] Youli Li, Roy Beck, Myung Chul Choi Tuo Huang, and Morito Divinagracia. Scatterless metal-single-crystal slit for small-angle $\mathrm{x}$-ray scattering and high-resolution $\mathrm{x}$-ray diffraction. J. Appl. Cryst., 41, 2008.

[68] Thomas M. Cover and Joy A. Thomas. Elements of Information Theory. John Wiley \& Sons, Chichester, 2006.

[69] David Albert Huffman. A method for the construction of minimum-redundancy codes. Proceedings of the I.R.E., September:1098, 1952.

[70] Aike Ruhlandt. Design und Berechnung eines kohärenten sub-20 nm fokus für das Strahlrohr P10 an PETRA III. Bachelorarbeit, Georg-August-Universität Göttingen, 2011.

[71] Pierre Thibault. Algorithmic methods in diffraction microscopy. PhD thesis, Cornell University, 2007.

[72] Martin Dierolf, A. Menzel, Pierre Thibault, P. Schneider, Cameron M. Kewish, R. Wepf, Oliver Bunk, and Franz Pfeiffer. Ptychographic x-ray computed tomography at the nanoscale. Nature, 467(7314):436-439, 2010. 
[73] Martin Dierolf, Pierre Thibault, A. Menzel, Cameron M. Kewish, K. Jefimovs, I. Schlichting, K. Von Koenig, Oliver Bunk, and Franz Pfeiffer. Ptychographic coherent diffractive imaging of weakly scattering specimens. New Journal of Physics, 12:035017, 2010.

[74] Björn Enders, Klaus Giewekemeyer, Thomas Kurz, Sergey Podorov, and Tim Salditt. Non-iterative coherent diffractive imaging using a phase-shifting reference frame. New Journal of Physics, 11:043021, 2009.

[75] Klaus Giewekemeyer. A study on new approaches in coherent x-ray microscopy of biological specimens. PhD thesis, Georg-August-Universität Göttingen, 2011.

[76] B. Abbey, L. W. Whitehead, H. M. Quiney, D. J. Vine, G. A. Cadenazzi, C. A. Henderson, Keith A. Nugent, E. Balaur, C. T. Putkunz, A. G. Peele, G. J. Williams, and lan McNulty. Lensless imaging using broadband x-ray sources. Nature Photonics, 5:420-424, 2011.

[77] Christian Morawe, Kathrin Friedrich, Markus Osterhoff, and Jean-Christophe Peffen. The new esrf multilayer facility: Progress and perspectives. SRI 2009, 10th International Conference on Radiation Instrumentation, 1234(1):720-723, 2010.

[78] Christian Morawe, Olivier Hignette, Peter Cloetens, W. Ludwig, Ch. Borel, P. Bernard, and Amparo Rommeveaux. Graded multilayers for focusing hard $x$ rays below $50 \mathrm{~nm}$. SPIE proceedings, 6317, 2006.

[79] Satio Takagi. A dynamical theory of diffraction for a distorted crystal. J. Phys. Soc. Jap., 26:1239-1253, 1969.

[80] Satio Takagi. Dynamical theory of diffraction applicable to crystals with any kind of small distortion. Acta Cryst., 15:1311-1312, 1962.

[81] Daniel Taupin. Prévision de quelques images de dislocations par transmission des rayons $X$ (cas de Laue symétrique). Acta Cryst., 23:25-35, 1967.

[82] Vito Mocella, Yves Epelboin, and Jean-Pierre Guigay. X-ray dynamical diffraction: the concept of a locally plane wave. Acta Crystallographica Section A, 56(3):308-316, 2000.

[83] Christian Morawe, Jean-Christoph Peffen, Eric Dufresne, Yong Chu, and Albert T. Macrander. Double gradient multilayers for broadband focusing. SPIE proceedings, 5195, 2003.

[84] Donald Erwin Knuth. The toilet paper problem. The American Mathematical Monthly, 91(8):465-470, 1984.

[85] Daniel H. Greene and Donald Erwin Knuth. Mathematics for the Analysis of Algorithms. Birkhäuser, Boston, 1990.

[86] Donald Erwin Knuth. The Art of Computer Programming, Volume 1, 3rd edition. Addison-Wesley, Boston, 1997.

[87] Charles Galton Darwin. The theory of x-ray reflection, part ii. Phil. Mag., 27:675-690, 1914. 
[88] Norio Kato. Pendellösung fringes in distorted crystals II. Application to two-beam cases. J. Phys. Soc. Jap., 19(1):67, 1964.

[89] William H. Zachariasen. Theory of X-Ray Diffraction in Crystals. Dover Publications, New York, 1945.

[90] André Authier. Dynamical theory of x-ray diffraction. Oxford University Press, Oxford, 2001.

[91] Zinovĭ Grigor'evich Pinsker. Dynamical Scattering of X-Rays in Crystals. Springer-Verlag, Berlin, 1978.

[92] Jürgen Härtwig. Hierarchy of dynamical theories of $x$-ray diffraction for deformed and perfect crystals. J. Phys. D: Appl. Phys., 34(10A), 2001.

[93] Heinrich Schlangenotto. Dynamische Theorie der Röntgenbeugung für deformierte Kristalle. Zeitschrift für Physik, 203(1):17-36, 1967.

[94] Yves Epelboin. Simulation of x-ray topographs. Materials Science and Engineering, 73:1-43, 1985.

[95] Yves Epelboin. A varying-step algorithm for numerical integration of Takagi-Taupin equations. Acta Crystallography Section A, 39(5):761-767, 1983.

[96] Jerzy Gronkowski. Propagation of x-rays in distorted crystals under dynamical diffraction. Physics Reports, 206(1):1-41, 1991.

[97] Vito Mocella, W. K. Lee., G. Tajiri, D. Mills, Claudio Ferrero, and Yves Epelboin. A new approach to the solution of the Takagi-Taupin equations for $\mathrm{x}$-ray optics: application to a thermally deformed crystal monochromator. Journal of Applied Crystallography, 36(1):129-136, 2003.

[98] Milton Abramowitz and Irene A. Stegun. Handbook of Mathematical Functions. Dover Publications, New York, 1972.

[99] Hanfei Yan. personal communication, 2009.

[100] Christian Morawe. personal communication, 2011.

[101] Raymond Barrett, Robert Baker, Peter Cloetens, Yves Dabin, Christian Morawe, Heikki Suhonen, Remi Tucoulou, Amparo Vivo, and Lin Zhang. Dynamically-figured mirror system for high-energy nanofocusing at the esrf. SPIE proceedings, 8139, 2011, in press.

[102] Dietrich Marcuse. Theory of dielectric optical waveguides. Academic Press, New York, 1974.

[103] Sebastian Panknin, Alexander K. Hartmann, and Tim Salditt. X-ray propagation in tapered waveguides: Simulation and optimization. Optics Communications, 281:2779-2783, 2008.

[104] Robert Hanbury Brown. The Wisdom of Science. Cambridge University Press, Cambridge, 1986.

[105] Joseph W. Goodman. Fourier Optics. Roberts \& Company Publishers, Greenwood Village, 2005. 
Sketch on page I: own work, based on painting by Balthasar Moncornet.

Photographies on pages 3,47, 49, and 93: own works.

Photography on page 19: own work; building design: Staatliches Baumanagement Niedersachsen.

Figure 2.2 own work, based on [53].

Figure 2.4 based on [61] (own publication).

Figure 3.5: measurements by Frank Siewert (BESSY, Berlin) and WinlightX (Pertuis).

Photography of figure 4.15: courtesy ESRF Grenoble.

Photography of figure 5.1: own work, experimental set-up: nano-focus end-station, P10.

IDL: trademark by Exelis Visual Information Solutions.

Intel, Xeon: trademarks by Intel Corporation.

Mathematica: trademark by Wolfram Research, Inc.

Matlab: trademark by The MathWorks, Inc. 


\section{Index}

adiabatic lenses, 20

anti-phase shift, 65, 66

average relative phase, 17

bender, 80,82

Born approximation, 47, 48, 51

boundary conditions, 66-68, 71, 73, 125, 131,132

Bragg's law, 47, 50, 55, 59, 62, 63, 65

Catalan number, 51, 113, 114

chaotic light source, 6, 9, 12

diffraction limit, 30, 34, 50, 81, 88, 92

distortion, 55, 56, 59, 61, 62, 64, 65

double-slit, 3-5

dynamical theory, 47, 48, 50, 51

elastic emission machining, 21

electron orbit, 4, 6, 7, 20

elliptical coordinates, 52, 53, 55, 59, 61, 66

entropy, 39

ergodic system, 9

ESRF, 49, 80, 81, 85

evanescent wave, 14, 21, 22, 24

fluctuation, $6,10,16,17$

fluctuations, 3, 6, 44

focus of a point-source, 29, 85, 88, 92

focus of extended source, 31, 34

Fresnel's coefficients, 22, 23, 71

Fresnel-Kirchhoff diffraction integral, 25, 125

fringes, 3, 5, 43, 46
Gaussian beam, 26

Gaussian-Schell model, 34, 35

geometrical optic, 3

GMP, 27

Goos-von Hänchen effect, 21, 22, 24, 25

Gouy phase, 5, 17, 22, 26, 43-45

GPGPU, 13

guided mode, 93, 95, 102

holography end-station, 28, 29

homogeneous medium, 58, 151

HTTP, 27, 127, 141, 145, 146

Huffman code, 39

Huygens' elementary sources, 25

ID22NI, 80-83, 85

improved slit model, 36-38, 40

integrated reflectivity, $73,85,87,89,122$, $124,130,133-135$

interference, $3,6,10,11,28,38,41,44$, 58

Kirkpatrick-Baez mirror, 14, 20, 42, 80, 82, 85

Kullback-Leibler divergence, 38-40

Laplacian, 54, 56, 57, 59

light ray, 3

linear absorption coefficient, 23, 97

local angle of incidence, 47, 61, 70, 130, 133, 135

local lattice vector, 54, 59

local rotation, $60,64,66,77,134$

magnetron sputtering, 49 
ML-500, 85, 87, 92

mode, $8,11,13,14,16,93-98$

modification factor, 65, 77, 79-83, 85, 87, $89,90,118,137$

modified Bragg condition, 49, 50, 52, 55, $60,62-66,77,80,87,133,134$, 137,149

MPI, 27

multilayer Laue lens, 40, 41, 51, 52

naïve slit model, 36,40

numerical aperture, 19, 30, 47, 50

Nyquist frequency, 17

obliquity factor, 26,125

occupation number, 16, 94-98

openMP, 27

oscillations, $75,77,80$

$P 10,22,24,25,28,29,31-35,42,43,46$, 48, 94

parameter scaling, 30, 34

Parratt's algorithm, 62, 70, 74-76, 135

partial coherence, 4-7, 11, 12, 16, 17, 21, $22,28,31,34-37,40-46,95,102-$ 105,121

PETRA III, 22, 28, 29, 34, 35, 42, 48, 94

phase trajectories, 44,45

phase unwrapping, 17, 44

physical limits, 85

propagation imaging, $14,15,42,104$

pseudo-rocking curve, 77, 79, 85, 89, 122

radiative mode, 93, 100-102

ray-tracing, 47, 48, 51, 113

readline, $27,127,139,141$

reciprocal lattice vector, 55

relative phase, 17

scatterless slits, 36

Snell's law, 23, 63, 124

spatial coherence, 4, 5, 7, 17, 44

speckle pattern, 9, 11
SSE, 27, 124

stationary field, 5

stencil, 69, 73, 131, 132

susceptibility, 53-55, 59, 71, 72, 133

synchrotron source, 4, 6, 7, 20, 97

Talbot interferometry, 4

temporal coherence, 4

toy model, 93, 95, 96, 98

transfocator, 20

two-beam approximation, 50, 51, 54, 55, 71

UPBL04, 80, 85, 86

van Cittert-Zernike theorem, 11, 12

visibility, 3-5, 8, 40, 41

volume diffraction, 47

wave-packet, 3, 6

waveguide, 4, 5, 11-17, 93-96, 98-105

weak conditions, 68

XMLS, 27, 115-117, 119, 122, 123, 127, $128,130,133,139,141,142,145-$ 147,151 


\section{List of Publications}

- Markus Osterhoff and Tim Salditt: Coherence filtering of $x$-ray waveguides: analytical and numerical approach, New Journal of Physics, 13(10):103026, 2011.

- Tim Salditt, Sebastian Kalbfleisch, Markus Osterhoff, Sven Philip Krüger, Matthias Bartels, Klaus Giewekemeyer, Henrike Neubauer, and Michael Sprung: Partially coherent nano-focused $x$-ray radiation characterized by Talbot interferometry, Optics Express, 19(10): 9656-9675, 2011.

- Sebastian Kalbfleisch, Henrike Neubauer, Sven Philip Krüger, Matthias Bartels, Markus Osterhoff, Dong-Du Mai, Klaus Giewekemeyer, Bastian Hartmann, Michael Sprung, and Tim Salditt: The Göttingen Holography Endstation of Beamline P10 at PETRA III/DESY, AIP Conf. Proc., 1365, 96-99, 2011.

- Markus Osterhoff and Tim Salditt: Partially coherent x-ray beam simulations: mirrors and more, Proc. SPIE Vol. 8141, Advances in Computational Methods for X-Ray Optics II, 2011.

- Christian Morawe and Markus Osterhoff: Hard X-Ray Focusing with Curved Reflective Multilayers, X-Ray Optics and Instrumentation, 2010:479631, 2010.

- Sebastian Kalbfleisch, Markus Osterhoff, Klaus Giewekemeyer, Henrike Neubauer, Sven Philip Krüger, Bastian Hartmann, Matthias Bartels, Michael Sprung, Olaf Leupold, Frank Siewert, and Tim Salditt: The holography endstation of beamline P10 at PETRA III, AIP. Conf. Proc., 1234, 433-436, 2010.

- Christian Morawe and Markus Osterhoff: Curved graded multilayers for X-ray nano-focusing optics, Nuclear Instruments and Methods in Physics Research Section A, 616:98-104, 2010.

- Markus Osterhoff and Tim Salditt: Real structure effects in X-ray waveguide optics: The influence of interfacial roughness and refractive index profile on the near-field and far-field distribution, Optics Communications 282(16):3250-3256, 2009. 


\section{Acknowledgement}

\section{Danksagung}

\section{Remerciements}

This thesis would not have been possible without close collaborations. At first, I acknowledge encouraging discussions with and stipulating questions from Prof. Dr. Tim Salditt. Undoubtful, Dr. Jean-Pierre Guigay contributed a great lot sharing his insights into the Takagi-Taupin theory. Merci beaucoup! Unforgotten are our often long and intense discussions, together with Dr. Claudio Ferrero. When needed, Dr. Christian Morawe contributed answers regarding practicalities of multilayer mirror design.

I thank my thesis committee, Prof. Dr. Tim Salditt, Prof. Dr. Arnulf Quadt, and Prof. Dr. Sarah Köster, for their advice in scientific practice and academic life. Thanks to Prof. Dr. Rainer G. Ulbrich for co-refereeing this thesis.

For many fruitful and clarifying discussions I am indebted to Dr. Cameron Kewish, Dr. Hanfei Yan, Dr. Jörg Maser, Prof. Dr. Christian Schroer, Dr. Ray Barrett, Dr. Jürgen Härtwig, and Tobias Liese.

Academic life was enriched by my office colleagues - Dr. Simon Castorph, Robin Wilke, and Tobias Reusch in Göttingen, Dr. Kathrin Friedrich in Grenoble. I enjoyed beamtimes with Sebastian Kalbfleisch, Matthias Bartels, Dr. Klaus Giewekemeyer, Dr. Sven-Philip Krüger, Henrike Neubauer, Dr. Christian Olendrowitz, many more - and also Prof. Dr. Tim Salditt. Thanks for Marius Priebe, Aike Ruhlandt, and Johannes Hagedorn for many productive hours; Jan Goeman, IRP system administrator, often helped quickly to solve problems.

Ganz besonderen Dank an Elisabeth, meinen Vater Werner Osterhoff und Elsbeth für die Wärme und den Zuspruch der vergangenen drei Jahre. 


\section{Lebenslauf}

Markus Osterhoff, geboren am 30. Juni 1983 in Warstein, Staatsangehörigkeit: deutsch.

\section{Wissenschaftlicher Werdegang}

Juni $2003 \quad$ Erlangung der Allgemeinen Hochschulreife, Gymnasium der Stadt Warstein

Oktober 2004 - Studium der Physik an der Georg-August-Universität

Dezember 2008 Göttingen

Dezember 2008 Diplom in Physik, Titel der Arbeit: „Numerische Modellierungen von Röntgenwellenleitern unter Berücksichtigung von Realstruktureffekten“; angefertigt am Institut für Röntgenphysik an der Georg-August-Universität Göttingen; Note: sehr gut

seit Januar 2009 wissenschaftlicher Mitarbeiter am Institut für Röntgenphysik, Georg-August-Universität Göttingen 

